Portland State University

PDXScholar

Fall 12-4-2019

\title{
Analysis of E-cigarette Liquids and Aerosols by NMR Spectroscopy: Compositions, Boiling Points, and Degradation Profiles
}

Anna Katherine Duell

Portland State University

Follow this and additional works at: https://pdxscholar.library.pdx.edu/open_access_etds

Part of the Chemistry Commons

Let us know how access to this document benefits you.

\section{Recommended Citation}

Duell, Anna Katherine, "Analysis of E-cigarette Liquids and Aerosols by NMR Spectroscopy: Compositions, Boiling Points, and Degradation Profiles" (2019). Dissertations and Theses. Paper 5351.

https://doi.org/10.15760/etd.7224

This Dissertation is brought to you for free and open access. It has been accepted for inclusion in Dissertations and Theses by an authorized administrator of PDXScholar. Please contact us if we can make this document more accessible: pdxscholar@pdx.edu. 
Analysis of E-cigarette Liquids and Aerosols by NMR Spectroscopy:

Compositions, Boiling Points, and Degradation Profiles

by

Anna Katherine Duell

A dissertation submitted in partial fulfillment of the requirements for the degree of

\author{
Doctor of Philosophy \\ in \\ Chemistry
}

\begin{abstract}
Dissertation Committee:
David H. Peyton, Chair

Robert M. Strongin

Steve L. Reichow

Erik J. Sánchez
\end{abstract}

Portland State University

2019 
(C) 2019 Anna Katherine Duell 


\begin{abstract}
Electronic cigarettes (e-cigarettes) are an increasingly popular tobacco product used by $\sim 3 \%$ of adults and $21 \%$ of $12^{\text {th }}$ grade students (as reported for the prior 30 -days in surveys) in the United States in 2018. As of 2019, this number went up to $25 \%$ for $12^{\text {th }}$ grade students. Due to how quickly e-cigarettes gained popularity and the rapid design changes that have ensued, there has not been enough time to accurately assess the effects of e-cigarettes, particularly for long-term use. The liquids used in e-cigarettes (eliquids) can degrade when vaped in an e-cigarette, so understanding the variables that can modulate degradation is useful for harm-reduction strategies. It is also useful to analyze various aspects of nicotine in e-liquids as this could influence the addictiveness of a product. This dissertation contains four manuscripts that broadly cover two categories of questions. 1) In order to evaluate the variables that can contribute to degradation in ecigarettes, a) the boiling points (i.e. aerosolization temperatures) of propylene glycol $(\mathrm{PG})+$ glycerol (GL) mixtures were determined (+ additives) and b) the effect of sucralose on aldehyde and hemiacetal formation via solvent degradation was explored. 2) Nicotine in e-liquids was analyzed in terms of a) protonation state (i.e. free-base vs monoprotonated), b) acid/nicotine ratio, and c) concentration, because these variables can alter the impact and addictiveness of a product. Primary findings in the four manuscripts include: 1) the boiling points of PG + GL mixtures were determined and ranged from $188.6{ }^{\circ} \mathrm{C}(\mathrm{PG})$ to $292{ }^{\circ} \mathrm{C}(\mathrm{GL})$. Parameterizations were determined using the GibbsKonovalov theorem so that the boiling point of a PG/GL mixture can be calculated for any PG/GL ratio. Mixture boiling points were also evaluated with additives $(2.5 \mathrm{~mol} \%$
\end{abstract}


water, $5 \mathrm{~mol} \%$ water, and $3 \mathrm{~mol} \%$ nicotine). Added water decreased the boiling points of all mixtures tested, and added nicotine decreased the boiling points of some PG/GL mixtures. 2) The addition of sucralose to e-liquids resulted in more aldehyde and hemiformal production via degradation when vaporized in an e-cigarette compared to identical sucralose-free e-liquids vaped under identical conditions. Sucralose was determined to be unstable to the vaping conditions in the e-cigarettes tested, and its degradation products then enhanced degradation of the solvents PG and GL. In manuscripts 3) and 4), the ratio of free-base nicotine relative to monoprotonated nicotine $\left(\alpha_{\mathrm{fb}}\right)$ was determined for a number of commercially available e-liquids including bottles ("salt" nicotine and "non-salt" nicotine), JUUL pods, and other JUUL-alikes/pods. Traditional e-liquids/pods and "salt" nicotine e-liquids/pods were evaluated for $\alpha_{\mathrm{fb}}$, $\mathrm{acid} /$ nicotine ratio, and nicotine concentration. $\alpha_{\mathrm{fb}}$ ranged from 0.00 to 0.98 for all commercial e-liquids tested, acid/nicotine ratio ranged from 0 to 4.03 by mol, and nicotine concentrations ranged from 3 to $62 \mathrm{mg} / \mathrm{mL}$ for tested e-liquids. Over time, eliquid manufacture has shifted from low nicotine concentration/high $\alpha_{\mathrm{fb}}$ content to higher nicotine concentration/lower $\alpha_{\mathrm{fb}}$ content (i.e. "salt" nicotine, such as JUUL and others), which results in a product that remains easily inhalable due to the reduced harshness, despite the relatively high nicotine content. 


\section{Acknowledgments}

I would like to thank the U.S. National Institutes of Health for funding this research through grant R01ES025257. The research herein was supported by the NIEHS and FDA Center for Tobacco Products (CTP). The content of this dissertation does not necessarily represent the views of the NIH or FDA.

I am indebted to Dr. David Peyton who has been an excellent mentor and adviser throughout my studies, shown me the art of compromise, and given me the opportunity to learn from him. I am grateful to my committee members, Drs. Robert Strongin, Steve Reichow, and Erik Sanchez who have provided valuable feedback and research recommendations for my dissertation throughout my time at Portland State University. I would also like to thank Dr. James F. Pankow who has invested a great deal of time in enhancing the quality of writing and content for many of the publications herein. I am appreciative of Drs. Mark Woods, Shankar Rananavare, and Albert Benight who have provided valuable advice and suggestions for my research, which I am appreciative of. I am also grateful to Dr. Theresa McCormick and other excellent professors.

The E-cigarette Research Group at Portland State University has been a valuable outlet to share data, discuss findings, and collaborate. I would like to thank Dr. Robert Jensen who developed the initial methods used to collect samples to study e-cigarettes and identified many of the degradation components produced by vaporizing propylene glycol + glycerol e-liquids. I am grateful to Rob for the time he spent teaching me what he had learned about e-cigarettes and e-liquid chemistry. I would also like to thank the following individuals and collaborators who have contributed to the E-cigarette Research 
Group: Dr. Wentai Luo, Dr. Jorge Escobedo Cordova, Michael Lethin, Kevin McWhirter, Kilsun Kim, Jiries Meehan-Atrash, Tetiana Korzun, Astrid Woodall, Shawna Vreeke, Ian Munhenzva, Dr. Jenny Zhu, James Salamanca, Chris Motti, Maryana Lazurko, and Robert Campbell.

I thank my Peyton Laboratory colleagues who have made the lab a pleasant place to be, including: Dr. Katherine Liebman, Elias Sibhatu, Dr. Kevin Murphy, Alyssa Palmer, and Dr. Cheryl Hodson.

I have appreciated the camaraderie of my cohort colleagues, especially Luke Lutkus, James Barnes, Colin Hiatt, Janette Myers, and Francis Dhoro.

Finally, yet importantly, I would like to thank my family and friends who have supported me in all that I do. Their support over the years has been invaluable. 


\section{Table of Contents}

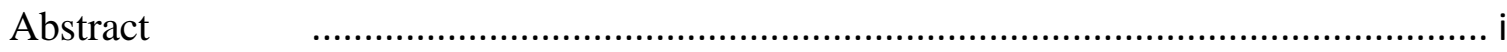

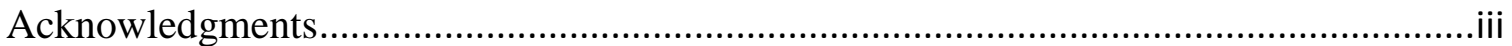

List of Tables

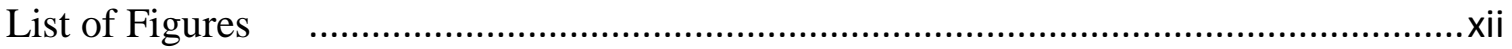

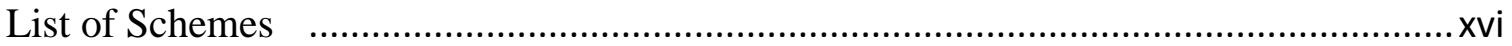

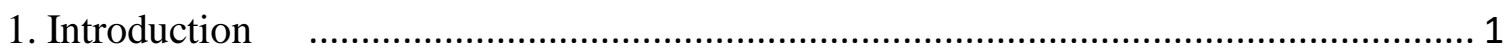

1.1. Background and History of Electronic Cigarettes....................................... 1

1.2. The Importance of E-liquids ......................................................................... 3

1.3. A Brief History of E-cigarette Research .................................................. 4

1.4. Fundamental Properties: Boiling Points of E-liquids................................... 12

1.5. Degradation of E-liquids and the Effect of Sucralose................................... 13

1.6. Protonation State of Nicotine: Free-base vs. Monoprotonated ......................... 15

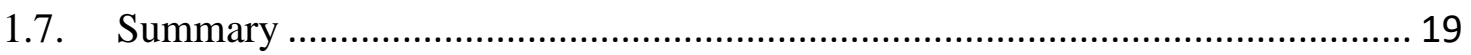

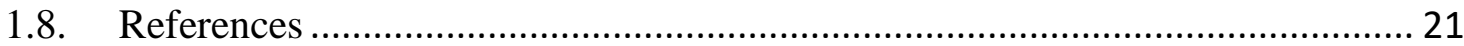

2. Boiling Points of the Propylene Glycol + Glycerol System at 1 Atmosphere Pressure: 188.6-292 ${ }^{\circ} \mathrm{C}$ Without and With Added Water or Nicotine .......................................... 26

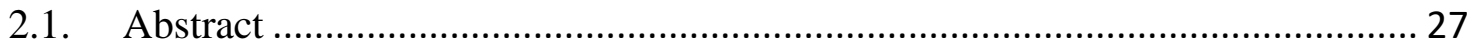

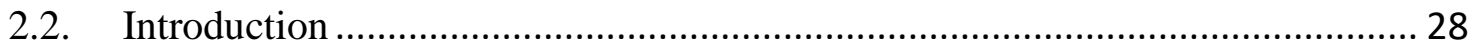

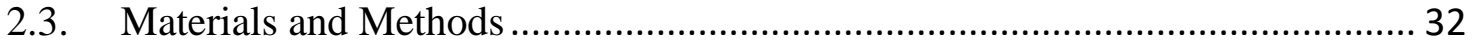

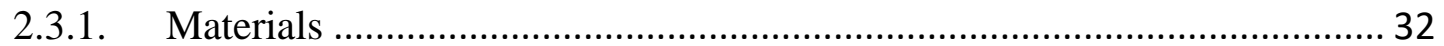

2.3.1.1. Boiling Point and ${ }^{1} \mathrm{H}$ NMR Testing ............................................. 32

2.3.1.2. Vaporized E-liquid Collection.................................................... 32

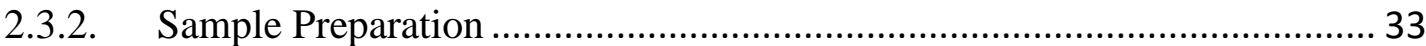

2.3.2.1. Large-scale Boiling Point Determinations ..................................... 33

2.3.2.2. Micro-scale Boiling Point Determinations ..................................... 33

2.3.2.3. All Boiling Point Samples ................................................................. 34

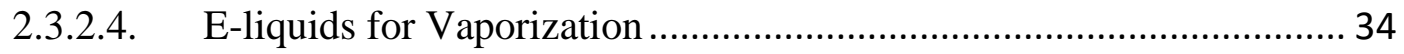

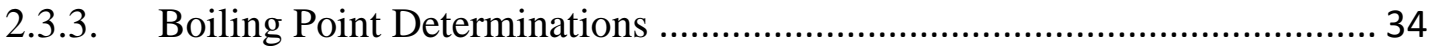




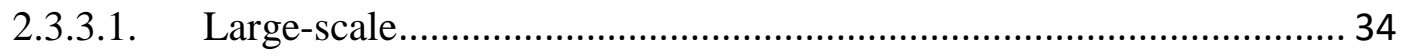

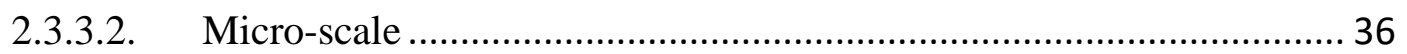

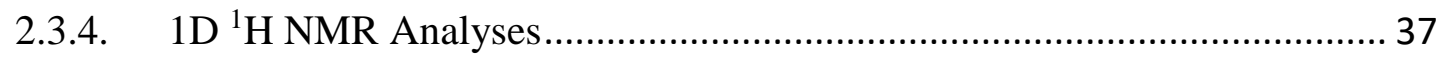

2.3.4.1. Large-scale Boiling Point Samples...................................................... 37

2.3.4.2. Micro-scale Boiling Point Samples ………………………………... 38

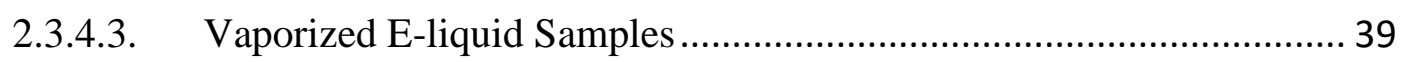

2.3.5. Vaporized E-liquid Sample Collection: the Effect of Added Water............ 39

2.3.5.1. E-cigarette Preparation ...................................................................... 39

2.3.5.2. E-cigarette Vapor Collection Setup ……………................................. 40

2.3.5.3. E-cigarette "Puff" Protocol.................................................................... 41

2.3.5.4. E-cigarette Vapor Collection at $22 \mathrm{~W}$............................................... 41

2.4. Results and Discussion......................................................................... 42

2.4.1. Large-scale Boiling Point Determinations .................................................. 42

2.4.1.1. Micro-scale Boiling Point Trials ........................................................ 46

2.4.1.2. The Effect of Water on Degradation in E-cigarettes ........................... 50

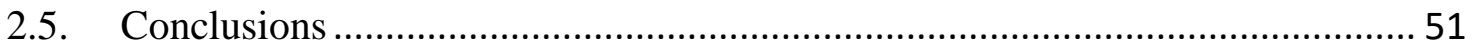

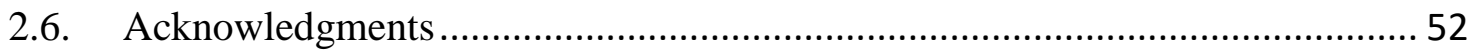

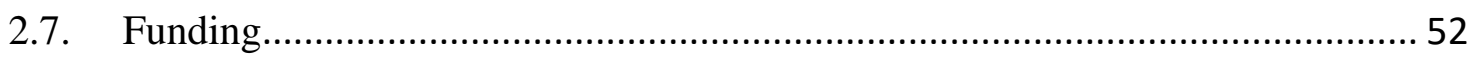

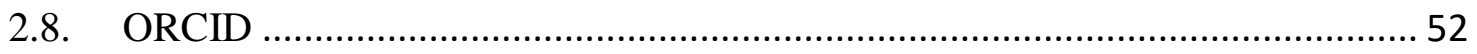

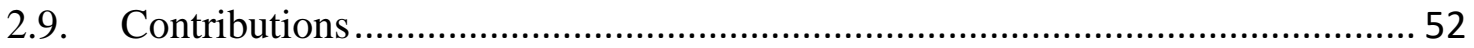

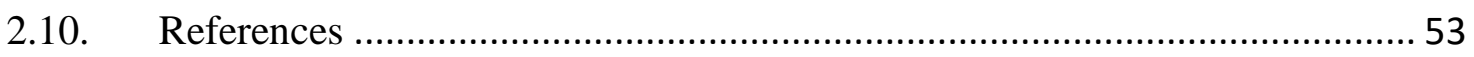

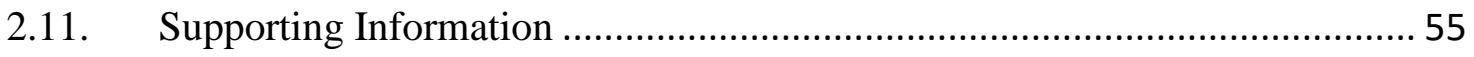

3. Sucralose-Enhanced Degradation of Electronic Cigarette Liquids during Vaping ...... 61

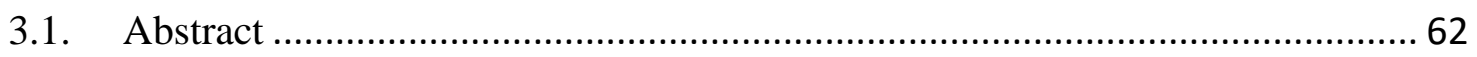

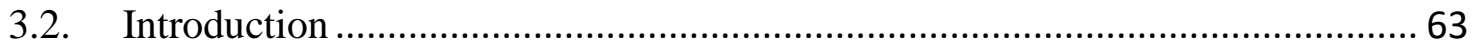

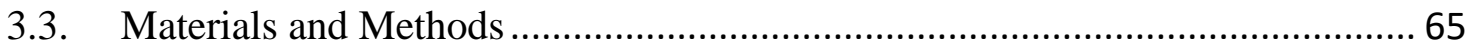

3.3.1. Chemicals and E-cigarette Devices ....................................................... 65

3.3.2. E-liquid Preparation with the Compositions Confirmed by ${ }^{1} \mathrm{H}$ NMR ......... 67

3.3.3. Sample Collection Protocol and ${ }^{1} \mathrm{H}$ NMR ................................................. 67 
3.3.4. Preparation of E-liquids, E-cigarette, and Calibration Standards for GC/MS,

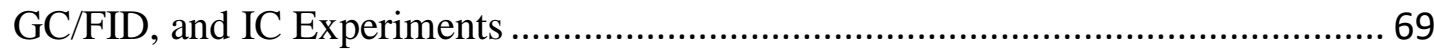

3.3.5. Sampling Methods for GC/MS, GC/FID, and IC ................................ 70

3.3.6. Analytical Methods for GC/MS, GC/FID, and IC ................................ 71

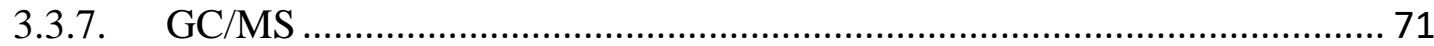

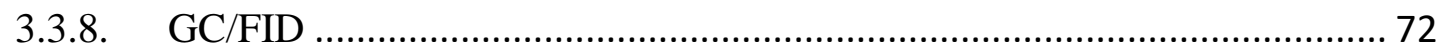

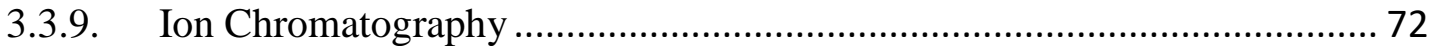

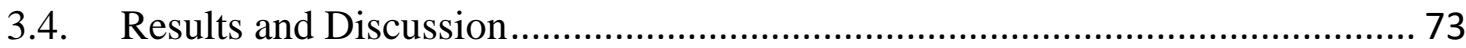

3.4.1. $\quad{ }^{1}$ H NMR of E-liquid Aerosol: Sample Variability, Device Differences, and

Degradation Products ....................................................................................... 73

3.4.2. Aerosol Collection and Target Analytes for GC/ MS, GC/FID, and IC

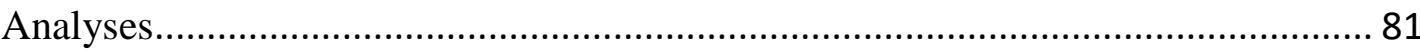

3.4.3. Forms of Chlorine Released from Sucralose ........................................ 87

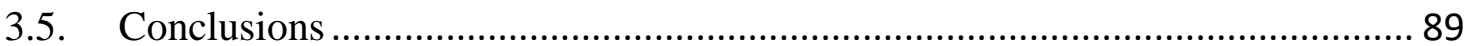

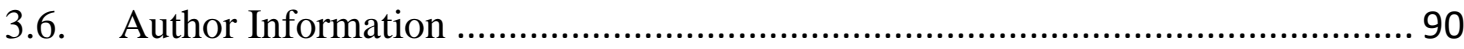

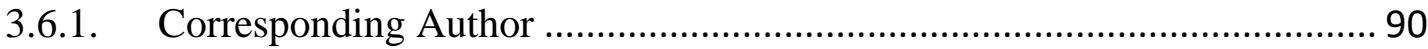

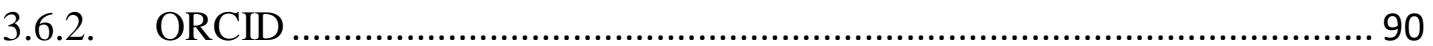

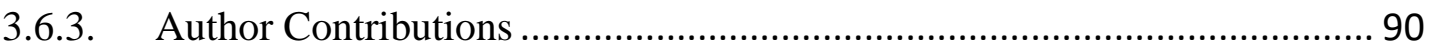

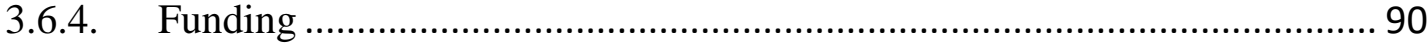

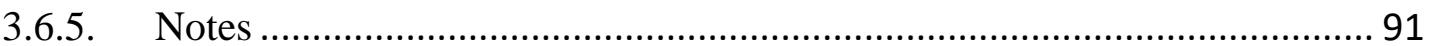

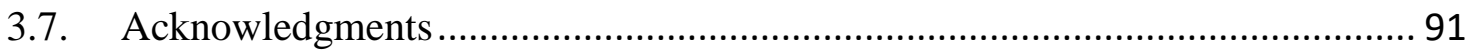

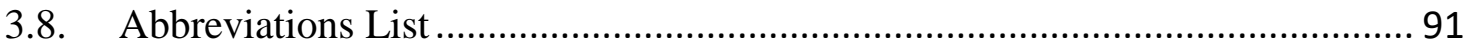

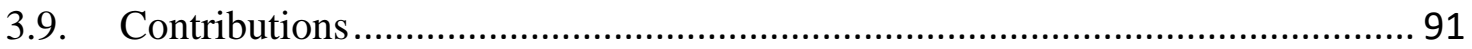

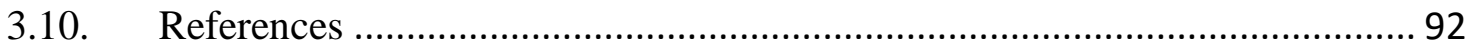

3.11. Supporting Information: Sucralose-enhanced Degradation of Electronic Cigarette Liquids During Vaping ..................................................................... 97

3.11.1. Isopropanol Hemiformal Determination .............................................. 98

3.11.2. Comparison of NMR Degradation Data with the Literature ...................... 99

3.11.3. Supplemental Material References ................................................... 104

4. Free-Base Nicotine Determination in Electronic Cigarette Liquids by ${ }^{1} \mathrm{H}$ NMR Spectroscopy 105 


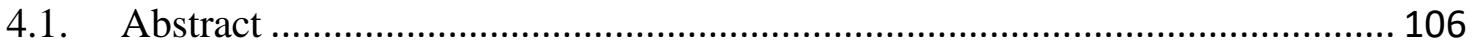

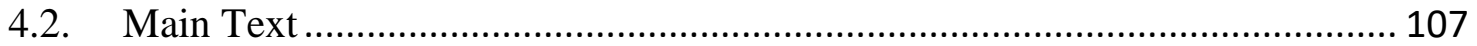

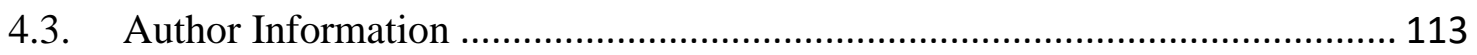

4.3.1. Corresponding Author ......................................................................... 113

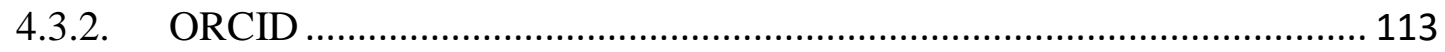

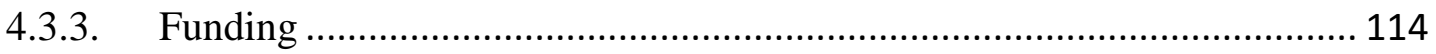

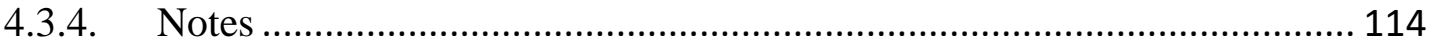

4.4. Acknowledgments ............................................................................. 114

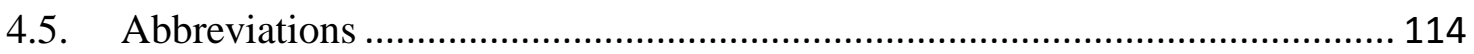

4.6. Contributions .......................................................................................... 114

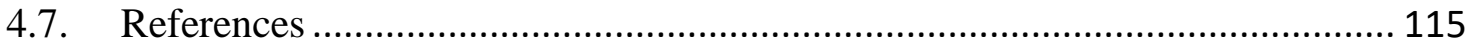

4.8. Supporting Information: Free-base Nicotine Determination in Electronic Cigarette Liquids by ${ }^{1} \mathrm{H}$ NMR Spectroscopy ........................................................... 117

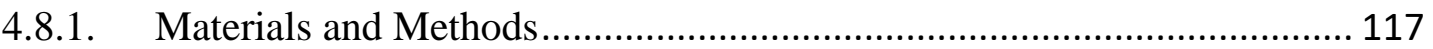

4.8.2. $\mathrm{pH}$ of Water Dilutions of Electronic Cigarette Fluids ............................... 119

4.8.3. Supporting Information References .......................................................... 124

5. Nicotine in Tobacco Product Aerosols: "It's Déjà Vu All Over Again" ...................... 125

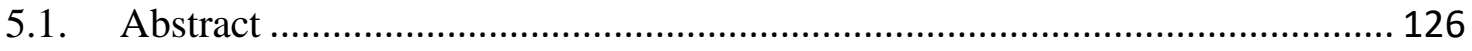

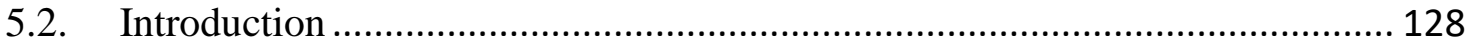

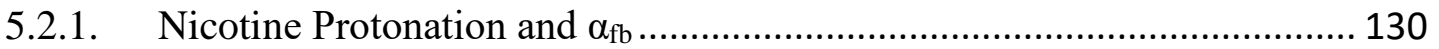

5.2.2. Net Protonation Reaction .................................................................... 131

5.3. Laboratory Methods …………................................................................. 133

5.3.1. NMR Determinations of $\alpha_{\mathrm{fb}}$, Nicotine and Acid Concentrations............... 133

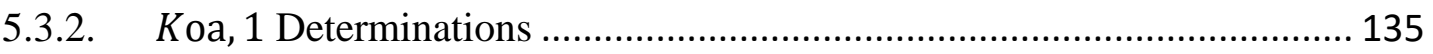

5.3.3. JUUL Aerosol PM Determinations........................................................ 139

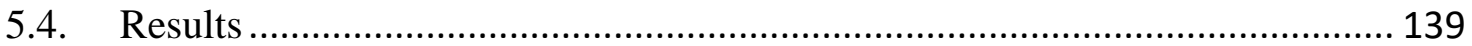

5.4.1. Protonation in E-liquids ..................................................................... 139

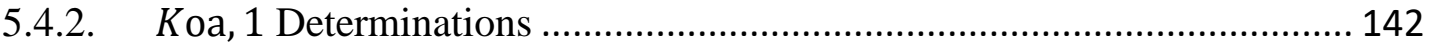

5.4.3. JUUL Aerosol PM Determinations......................................................... 144

5.5. Discussion ....................................................................................... 144 
5.5.1. Past was Prologue: Vu - Tobacco Smoke

5.5.2. Present: 'Déjà Vu' - E-cigarette Aerosols

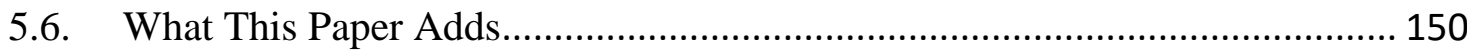

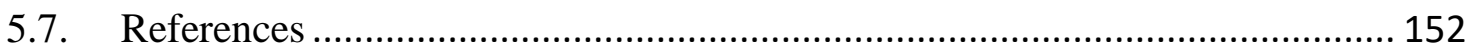

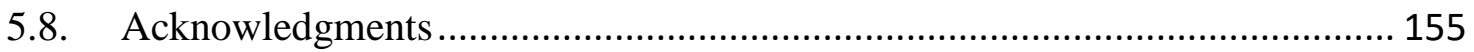

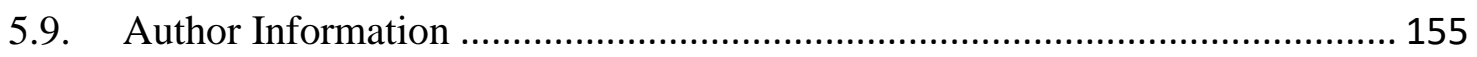

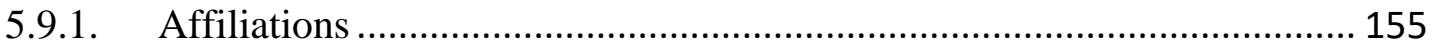

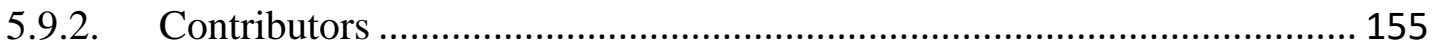

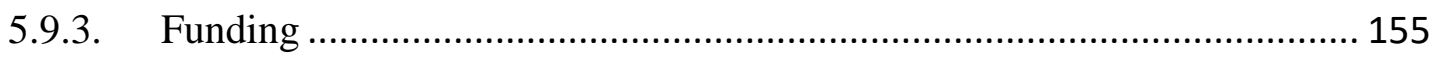

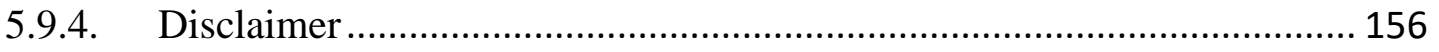

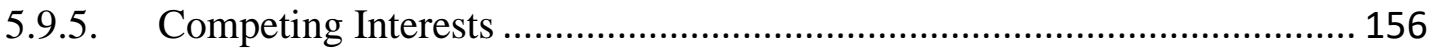

5.9.6. Patient Consent for Publication ............................................................. 156

5.9.7. Provenance and Peer Review ........................................................... 156

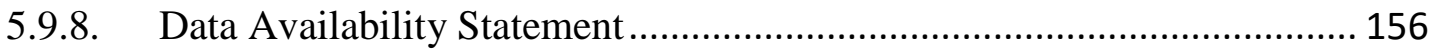

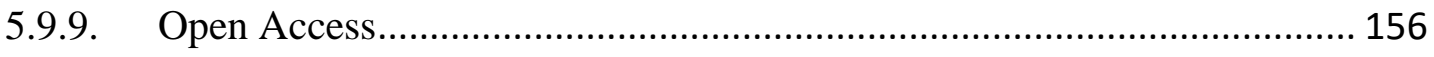

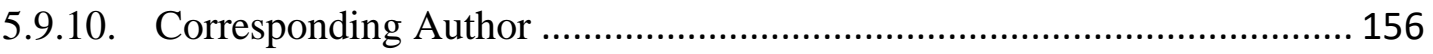

5.10. Supporting Information: Nicotine in Tobacco Product Aerosols: "It's Déjà Vu

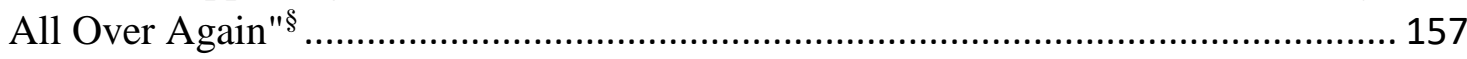

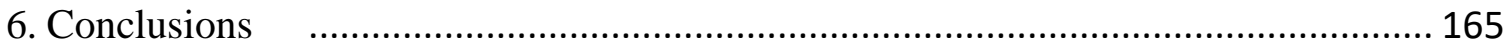

6.1. Boiling Points of the Propylene Glycol + Glycerol System at 1 Atmosphere Pressure: $188.6-292{ }^{\circ} \mathrm{C}$ Without and With Added Water or Nicotine ${ }^{1}$...................... 166

6.2. Sucralose-Enhanced Degradation of Electronic Cigarette Liquids during

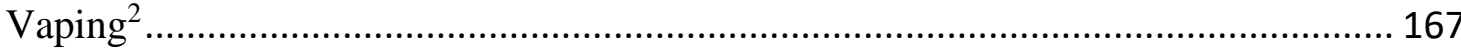

6.3. Free-Base Nicotine Determination in Electronic Cigarette Liquids by ${ }^{1} \mathrm{H}$ NMR

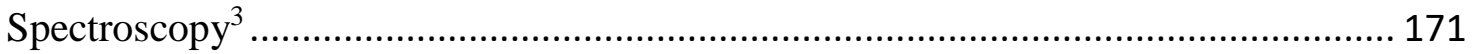

6.4. Nicotine in Tobacco Product Aerosols: "It's Déjà Vu All Over Again"4 ........ 174

6.5. Overall Conclusions ......................................................................... 178

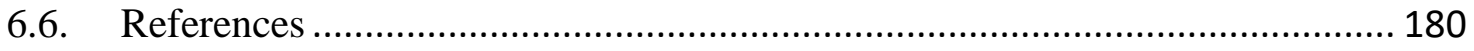




\section{List of Tables}

\section{Chapter 2}

Table 1. Antoine Equation Parameters for Vapor Pressure $\mathrm{p}^{\mathrm{o}}$ (bar) of the Pure Liquids Propylene Glycol (PG) and Glycerol (GL), with Applicable Temperature Ranges, $\log _{10}\left(\mathrm{p}^{\mathrm{o}}\right)=\mathrm{A}-\mathrm{B} /(\mathrm{T}(\mathrm{K})+\mathrm{C}) .(1 \mathrm{~atm}=1.01325$ bar. $)$ http://webbook.nist.gov/chemistry/

Table 2. Boiling Point Values at 1 Atm Pressure Assuming Raoult's Law (Ideal

Mixtures). $\quad p_{\mathrm{TOT}}(T)=x_{\mathrm{PG}} p_{\mathrm{PG}}^{\mathrm{o}}+x_{\mathrm{GL}} p_{\mathrm{GL}}^{\mathrm{o}}=1.01325 \mathrm{bar}(=1 \mathrm{~atm})$ For Antoine Equation

Parameters in Table 1.

Table 3. Measured Boiling Point (BP) Values of Propylene Glycol (PG) and Glycerol (GL) Mixtures With Volume \%, Weight \%, and Mol \% (N=3).

Table 4. Coefficients Determined for Gibbs-Konovalov Parameterization of Propylene Glycol (PG) and Glycerol (GL) Boiling Point Data.

Table 5. Calculated Boiling Point (BP) Values $\left({ }^{\circ} \mathrm{C}\right)$ for Propylene Glycol and Glycerol (GL) Mixtures by Volume, Weight, and Mole Percent GL using Coefficients A-D in Table 4, and Equation 2.

Table 6. Micro-Scale Boiling Points (BP) of Propylene Glycol (PG) + Glycerol (GL) Mixtures with Additives in Relation to Mol\% GL (Relative to Moles PG Only). …...... 48

Table S 1. Comparison Between Pre- and Post-Boiling Compositions in terms of Percent Glycerol (GL) for Propylene Glycol (PG) + GL Mixtures. Average Percent GL, N=3, as

Determined by NMR $(\% \mathrm{PG}=100 \%-\% \mathrm{GL})$. 55

Table S 2. Boiling point variability for PG+GL mixtures between trials. 56

Table S 3. Difference between the boiling points proposed by the Antoine fit, and calculated values generated using the Gibbs-Konovalov parameterization 57

Table S 4. The absolute value of the difference between the experimentally determined boiling points (average (AVE) \pm SD) and the Gibbs-Konovalov calculated values. The Gibbs-Konovalov parameters used can be found in Table 4. The average of the absolute values of the differences is $0.11^{\circ} \mathrm{C}$. The standard deviation of these values is $0.13{ }^{\circ} \mathrm{C} . .60$

\section{Chapter 3}

Table 1. Compounds detected ( $\mu \mathrm{g}$ per mg aerosol condensate). 
Table 2. Total chlorine found from samples containing sucralose.

\section{Chapter 4}

Table S-1. Measured pH values in water dilutions of e-liquids by Lisko et al. (2015), and calculated values for the post-dilution nicotine concentrations with and without consideration of equilibrium with atmospheric $\mathrm{CO}_{2}$.

\section{Chapter 5}

Table 1. Listed vs. measured nicotine contents, molar acid/nicotine ratios, and free-base fraction $\left(\alpha_{\mathrm{fb}}\right)$ for a selection of JUUL pod liquids, 'look-a-like/knockoff' pod liquids, and bottled e-liquids (additional details can be found online in supplementary table S-1) $\cdots 136$

Table 2. Values of $K_{\mathrm{oa}, 1}\left(40^{\circ} \mathrm{C}\right)$ for benzoic acid (BA) and vanillin in e-liquid formulations

Table S-1 Full table of values for listed vs. measured nicotine contents, molar acid/nicotine ratios (all determined by ${ }^{1} \mathrm{H}$ NMR), and free-base fraction determinations $\left(\alpha_{\mathrm{fb}}\right)$ for a selection JUUL pod liquids, "look-a-like/knockoff" pod liquids, and bottled e-liquids. 


\section{List of Figures}

\section{Chapter 1}

Figure 1. Use of cigarettes and e-cigarettes by high school students in the past 30 days in the United States.

Figure 2. Common e-liquid components, from left to right, propylene glycol, glycerol, and nicotine. 4

Figure 3. Structures for (A) sucrose versus (B) sucralose. …………………………. 14

Figure 4. Nicotine can exist in three protonation states depending on the $\mathrm{pH} . \cdots \cdots \cdots \cdots \cdots \cdots \cdots$

Figure 5. Selected regions of a ${ }^{1} \mathrm{H}$ NMR spectrum showing the nicotine resonances of interest in this work.

\section{Chapter 2}

Figure 1. Schematic of Boiling Point (BP) Setup. Thermometer in third port represents a digital thermometer.

Figure 2. Averages of Triplicate Boiling Point Measurements for Mixtures Composed of Propylene Glycol (PG) and Glycerol (GL). Mole percent GL post-boiling was determined by NMR analysis. Error bars as $\pm 1 \mathrm{SD}$ are too small to be seen; the largest SD is 0.6. $\cdot 44$

Figure 3. Averages of Triplicate Boiling Point Measurements for Mixtures Composed of Propylene Glycol (PG) and Glycerol (GL). The boiling points of propylene glycol + glycerol mixtures were determined in the absence of additives ("Large-scale boiling: no additives"), as well as in the presence of $3 \mathrm{~mol} \%$ nicotine, $2.5 \mathrm{~mol} \%$ added water, and 5 $\mathrm{mol} \%$ added water. Fits are included to guide the eye, rather than allow extrapolated boiling points. Error $( \pm 1 \mathrm{SD})$ is provided for all data points $(\mathrm{N}=3)$.

Figure S 1. ${ }^{1} \mathrm{H}$ NMR spectra showing degradation products produced by vaporizing PG:GL e-liquid containing varying amounts of water using three different coils. The following PG+GL degradation products can be identified using the chemical shifts of these products outlined by Jensen et al. (2017): propanal ( $\delta 9.68$, triplet), acetaldehyde ( $\delta$ 9.65 , quartet), glyceraldehyde ( $\delta 9.62$, doublet), glycolaldehyde ( $\delta 9.61$, singlet), lactaldehyde ( $\delta 9.58$, doublet), acrolein ( $\delta 9.56$, doublet), and three different PG or GLbased formaldehyde hemiacetals ( $\delta 6.12$, triplet; $\delta 6.18,2$ overlapped triplets; $\delta 6.12$, triplet). 


\section{Chapter 3}

Figure 1. Sample degradant variability using the same coil with and without sucralose

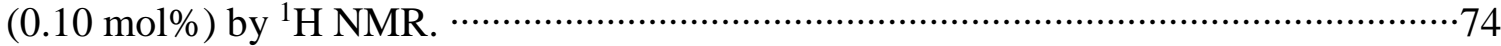

Figure 2. Degradant variability for 3 coils (A, B, \& C), all at $0.10 \mathrm{~mol} \%$ sucralose by ${ }^{1} \mathrm{H}$ NMR.

Figure 3. Increased sucralose concentrations generated a greater concentration of aldehydes using the same device, coil, and vaping conditions by ${ }^{1} \mathrm{H}$ NMR.

Figure 4. Increased sucralose concentrations generated a greater concentration of hemiacetals using the same device and coil by ${ }^{1} \mathrm{H}$ NMR.

Figure 5. Propylene glycol- and glycerol-based hemiacetal production, ${ }^{23}$ other degradation products identified in this study, and related structures.

Figure 6. Fraction of nicotine in the free-base state $\left(\alpha_{\mathrm{fb}}\right)$ in e-liquids (PG, GL, and nicotine) before and after being vaporized with and without sucralose $(0.12 \mathrm{~mol} \%)$ as determined using differences in ${ }^{1} \mathrm{H}$ NMR chemical shifts.

Figure S1: $\mu \mathrm{g}$ acetals and hemiacetal per mg aerosol condensate detected in each vial as detected by $\mathrm{GC} / \mathrm{MS}$ or GC/FID generated using a $1.2 \Omega$ coil at 20 watts containing 1:1 molar propylene glycol and glycerol.

Figure S2: Chlorinated targets detected in each vaporized sample vial as detected by GC/MS, GC/FID, or IC generated using a $1.2 \Omega$ coil at 20 watts containing $1: 1$ molar PG and GL.

Figure S3: NMR integration values as a percent of the propylene glycol in each sample.

Figure S4: $\mu \mathrm{g}$ of each degradation component per $\mathrm{mL}$ of e-liquid consumed calculated from NMR integration values.

Figure S5: ng of each degradation component per puff.

\section{Chapter 4}

Figure 1. The distribution of nicotine in tobacco and vape aerosols primarily involves two forms; left) Nic (free-base) which has volatility; and right) $\mathrm{NicH}^{+}$(monoprotonated) which is non-volatile. The fraction of the free-base for $\left(\alpha_{\mathrm{fb}}\right)$ depends on the acid/base conditions.

Figure 2. ${ }^{1} \mathrm{H}$ NMR spectra showing the chemical shifts changes for nicotine in a PG+GL stock mixture with the addition of acid and base, independently. 
Figure 3. Free-base nicotine fraction $\left(\alpha_{\mathrm{fb}}\right)$ in commercial e-liquids as an average using aromatic protons $\mathrm{H}_{\mathrm{a}}$ and $\mathrm{H}_{\mathrm{b}}$. The ranges between chemical shift differences are indicated.

Figure S-1. Measured pH values in water dilutions of e-liquids by Lisko et al. (2015), compared to predicted values for the post-dilution nicotine concentrations with and without consideration of equilibrium with atmospheric $\mathrm{CO}_{2}$.

\section{Chapter 5}

Figure 1. Top: the distribution of nicotine in vape and tobacco aerosols primarily involves two forms; centre: $\mathrm{NicH}^{+}$(monoprotonated) which is non-volatile; and right: free-base (fb) nicotine, which is volatile. The fraction of $\mathrm{fb}\left(\alpha_{\mathrm{fb}}\right)$ depends on the $\mathrm{acid} / \mathrm{b}$ ase conditions. In water at $25^{\circ} \mathrm{C}, \mathrm{p} K_{\mathrm{a}, 2}=8.01$. Bottom: so-called 'nicotine salts' in electronic cigarette liquids are formed by adding an organic acid (benzoic acid is depicted here) to the formulation, producing a lower $\alpha_{\mathrm{fb}}$ that depends on the ratio of nicotine:acid, as well as temperature and solvent conditions.

Figure 2. The expected harshness of a nicotine- containing product is influenced by both the free-base fraction $\left(\alpha_{\mathrm{fb}}\right)$ and the total nicotine concentration $\left(\mathrm{Nic}_{\mathrm{tot}}\right)$

Figure 3. A visual representation of the historical changes in $\alpha_{\mathrm{fb}}$ in tobacco smoke $\mathrm{PM}^{27}$ (top) in comparison to how electronic cigarette fluids and their associated aerosols have been changed (bottom). fc, flue- cured; $\alpha_{\mathrm{fb}}$, fraction of nicotine in the free- base form; $\mathrm{M}$, Marlboro; Nic, nicotine; OA, organic acid; PM, particulate matter.

Figure S-1. Comparisons between listed and actual nicotine weight-\% contents for a selection of e-liquids. The listed amounts are shown by grey bars, while the actual amounts (as assessed by ${ }^{1} \mathrm{H}$ NMR integrations converted to weight-\% values) are shown by black bars.

Figure S-2. The ratio of mol-\% acid / mol- $\%$ nicotine for e-liquids containing benzoic acid or levulinic acid. Note that the ZOOR product was found not contain any benzoic acid (but did contain levulinic acid; indicated as striped bar), all other products with a positive acid/nicotine ratio contained benzoic acid, and the JUUL products are all essentially 1:1 benzoic acid/nicotine. JUUL 3 weight\% e-liquids are shown as checkered bars. 
Figure S-3. The $\alpha_{\mathrm{fb}}$ values for the selection of e-liquids sampled. The two products from JUUL with 3 weight- $\%$ nicotine, as opposed to with 5 weight- $\%$ in the rest of the JUUL products are shown as checkered bars. These values are the averages (when possible) of the determinations available from the multiple aromatic resonances from the nicotine molecules, as explained in Materials and Methods. For some products, only one, rather than two, $\alpha_{\mathrm{fb}}$ value was able to be calculated (See Table S-1). Note how these values correlate strongly with the inverse of the benzoic acid / nicotine ratios shown in Figure 2.

Figure S-4. The $\alpha_{\mathrm{fb}}$ values from Figure 3, multiplied by the nicotine $\mathrm{mg} / \mathrm{mL}$ values. The resulting numbers permit a rough comparison of the total free-base nicotine exposure that a user would obtain from vaping the same weight of aerosol from each product. Note that the benzoic acid variation across these pods has a strong influence on the total exposure to free-base nicotine. The two products from JUUL with 5 weight- $\%$ nicotine, as opposed to with 3 weight- $\%$ in the rest of the JUUL products are shown as checkered bars. $\cdots \cdots 160$

Figure S-5. Samples for composition analysis were prepared by adding a drop of each eliquid to DMSO- $d_{6}$ and testing by ${ }^{1} \mathrm{H}$ NMR. Spectra were normalized relative to the nicotine resonances because the e-liquids contained different ratios of propylene glycol:glycerol. Here, it can be seen that the ZiiP "Cappuccino" e-liquid has a much higher benzoic acid:nicotine ratio (4:1) than the JUUL "Mango" benzoic acid:nicotine ratio $(1.1: 1)$. 


\section{List of Schemes}

\section{Chapter 1}

Scheme 1. Thermal degradation pathways for propyene glycol (PG) in the presence of oxygen. Scheme from Jensen et al. (2017). ${ }^{15}$ Labelled compounds shown include: (3a) (Z)-prop-1-en-1-ol, (3b) (E)-prop-1-en-1-ol, (4) dihydroxyacetone, (6) lactaldehyde, (9) acetaldehyde, (10) propanal, (11) acetone, (12) hydroxyacetone (acetol), (13) acetic acid,

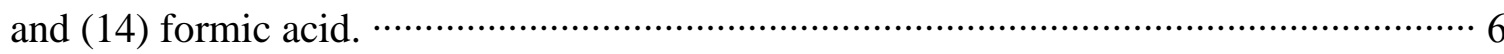

Scheme 2. Thermal degradation pathways for glycerol (GLY) in the presence of oxygen. Scheme from Jensen et al. (2017). ${ }^{15}$ Labelled compounds shown include: (2) glycidol, (4) dihydroxyacetone, (5) acrolein, (7) glycolaldehyde, (8) glyceraldehyde, (9) acetaldehyde, (12) hydroxyacetone (acetol), (13) acetic acid, and (14) formic acid.

Scheme 3. Formation of hemiacetals from propylene glycol or glycerol reacting with formaldehyde. 


\section{Introduction}

\subsection{Background and History of Electronic Cigarettes}

The modern invention of electronic cigarettes (e-cigarettes) has been credited to Hon Lik, a Chinese pharmacist, who first filed a patent for an e-cigarette in $2003 .{ }^{1}$ While this is technically the start of the present e-cigarette, it took years for e-cigarettes to gain popularity in the United States. E-cigarettes started to gain traction in the United States around $~ 2010$, with e-cigarette sales doubling from 2012 to 2013 (sales increased from $\$ 274$ million to $\$ 636$ million over this period $)^{2}$ and the quantity of e-cigarette sales generally increased over time. ${ }^{3}$ Adult e-cigarette use in the United States was estimated at $3.2 \%$ in $2018 .{ }^{4}$ Furthermore, from 2017 to 2018 , a $~ 0.5-1 \%$ increase in daily ecigarette use by those between the ages of 18-44 was observed, while other age groups (45+) remained similar or decreased, indicating that trends in e-cigarette use differ by age. ${ }^{4}$ E-cigarette use in the United States by $12^{\text {th }}$ grade students in a "past 30 days" survey was estimated at 11,12 , and $21 \%$ of students over the years of 2016,2017 , and 2018, respectively, as reported by Gentzke et al. (Figure 1). ${ }^{5}$

Since modern e-cigarettes were introduced, e-cigarettes and the associated electronic cigarette liquids (e-liquids) have gone through a number of changes. Initial products attempted to imitate traditional cigarettes in terms of their appearance (i.e. cig-alikes), while other models were simple tank-style devices composed of an e-liquid tank connected to a power source/heating element. Over time, more complex and customizable devices such as sub-ohm atomizers, drippers, squonk mods, and others have entered the market. ${ }^{6}$ Many of these products allowed the consumer to change the coil 
resistance, power, wattage, etc. - allowing for even greater variation in the e-cigarette products available. Regardless of specific design, e-cigarettes are generally composed of a power source that provides energy to a heating coil. The coil is positioned in the eliquid, typically possessing a wicking material to draw e-liquid into the coil. The coil vaporizes the e-liquid and the resulting aerosol travels through a mouthpiece to the consumer.

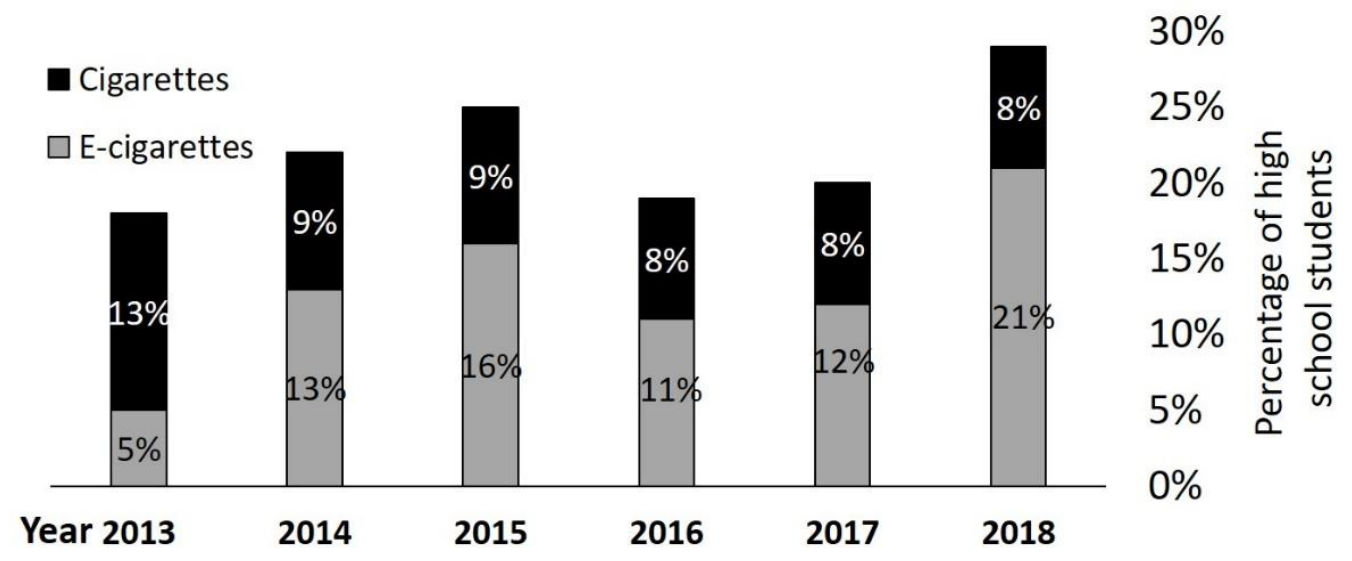

Figure 1. Use of cigarettes and e-cigarettes by high school students in the past 30 days in the United States.

There has been a shift towards less complex and perhaps more accessible products over the last few years. For example, the popularity of pod-based e-cigarettes (e.g. JUUL and others) has surged in recent years. ${ }^{7}$ These devices are characterized by a lack of customizable aspects; these devices simply require that a commercially available pod full of e-liquid be inserted into the device, and that the device be charged regularly. Podbased systems typically do not have a power button, but rather activate automatically upon the application of negative pressure (i.e. inhalation) to the mouthpiece. 
The simplicity of these devices reduces the "barrier to entry" for consumers as knowledge of electronics (i.e. relationship between resistance, power, and voltage), decisions about battery power, and coil selection are unnecessary. JUUL, which exemplifies this shift towards simpler products as it has no settings, was the most popular e-cigarette device by 2017 (in the United States by market share) ${ }^{3}$ and continues to lead the e-cigarette market.

\subsection{The Importance of E-liquids}

Regardless of device design, all current e-cigarettes rely on the use of e-liquids. These liquids are predominantly composed of carrier solvents, typically mixtures of propylene glycol (PG) and/or glycerol (GL), which serve to deliver nicotine and/or flavorants (Figure 2). A broad variety of commercially available e-liquids exist. Aspects of e-liquids that can be modified include: PG/GL ratio, nicotine concentration, flavorant blend/concentration, and various additives. Sometimes additives (often referred to as flavor enhancers) are combined with e-liquids to augment aspects of the vaping experience as desired by the consumer. Commercially available e-liquid additives include sweeteners (sucralose, erithritol, ethyl maltol, etc.), menthol/mint/cool sensation (e.g. "Koolada" or menthol), "sour" flavor, triacetin, and others.

Herein, the focus on e-liquid composition is in relation to either a) the effect(s) on degradation production during vaping (i.e. effect of an individual component on degradation production trends), and/or b) the possible effects on addiction in humans (i.e. due to nicotine concentration and/or protonation state). 


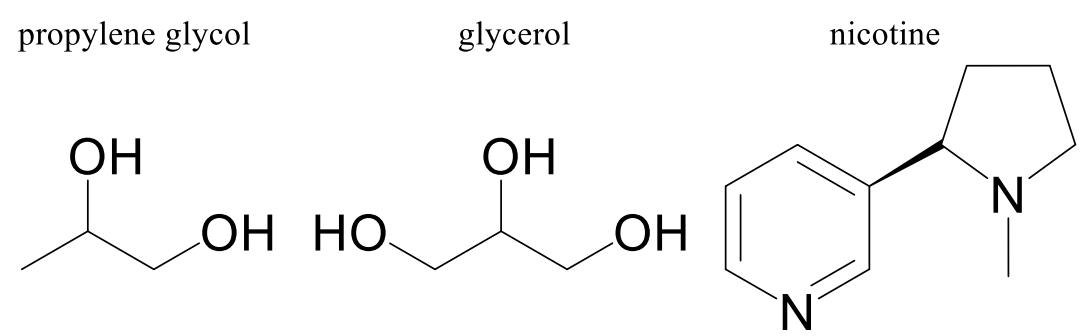

Figure 2. Common e-liquid components, from left to right, propylene glycol, glycerol, and nicotine.

\subsection{A Brief History of E-cigarette Research}

A variety of instrumentation techniques can be applied to studying e-cigarettes and their chemistry. For studying flavorants/volatile components in e-liquids, particularly unknown compounds, gas chromatography-mass spectrometry (GC/MS) can be particularly useful, especially when paired with a compound reference library. ${ }^{8,9,10,11}$ The sensitivity that can be achieved with GC/MS and liquid chromatography-mass spectrometry (LC/MS) is also excellent for detecting, identifying, and quantifying trace amounts of compounds such as nicotine, aldehydes, and other contaminants. ${ }^{12,13}$ Other instrumentation techniques that can be useful for analyzing e-liquids include: inductively coupled plasma mass spectrometry (ICP-MS) for metals, high-performance liquid chromatography + spectrophotometric detector (HPLC-DAD) for carbonyls, and NMR spectrometry for delicate compounds that might be destroyed using other techniques. ${ }^{13,14}$

The exploration of modern e-cigarette solvent chemistry was pioneered by Jensen et al. (2017) ${ }^{15}$ among others, wherein some of the main degradation products that can be produced by vaping mixtures of just PG/GL (i.e. primary carrier solvents in e-liquids) were outlined. Compounds that can be produced as a result of vaping a PG/GL mix 
(Scheme 1 and Scheme 2) include: aldehydes (e.g. acetaldehyde, propanal, glyceraldehyde, glycolaldehyde, acrolein, lactaldehyde, (Z)-prop-1-en-1-ol, (E)-prop-1en-1-ol), alcohols (e.g. glycidol, allyl alcohol), ketones (e.g. dihydroxyacetone, hydroxyacetone, acetone), acids (e.g. acetic acid, formic acid) and formaldehyde releasers (e.g. PG hemiacetals and GL hemiacetals; Scheme 3). ${ }^{15}$ Carbonyl degradation products are generally classified as volatile organic carbonyls (VOCs). Other degradation products of possible concern include: reactive oxygen species, ${ }^{16}$ furans (e.g. furfural, 5hydroxymethylfurfural), ${ }^{17}$ and metals ${ }^{18}$. Many of these variables (with the likely exception of metals) can be moderated by e-liquid components. For example, in general, flavorants enhance degradation production. ${ }^{19}$

Jensen et al. (2015) were also the first to publish on the quantification of formaldehyde and formaldehyde + PG/GL hemiacetals in e-cigarette aerosols $;{ }^{20}$ others found formaldehyde in the gas phase of e-cigarette aerosols but probably missed these hemiacetals. ${ }^{13,21,22}$ To complicate the study of formaldehyde in e-cigarette aerosols, formaldehyde can also react with other components in the aerosol (such as PG or GL) to form additional structures, which can be referred to as "formaldehyde releasers", hemiacetals, or hemiformals. ${ }^{15,20}$ These components need to be analyzed in addition to 'free' (largely gas-phase) formaldehyde to determine the total quantity of formaldehyde produced during vaping. However, they can be difficult to quantify because free formaldehyde in the aerosol rapidly converts to PG/GL hemiacetals (and possibly other compounds) over time. 


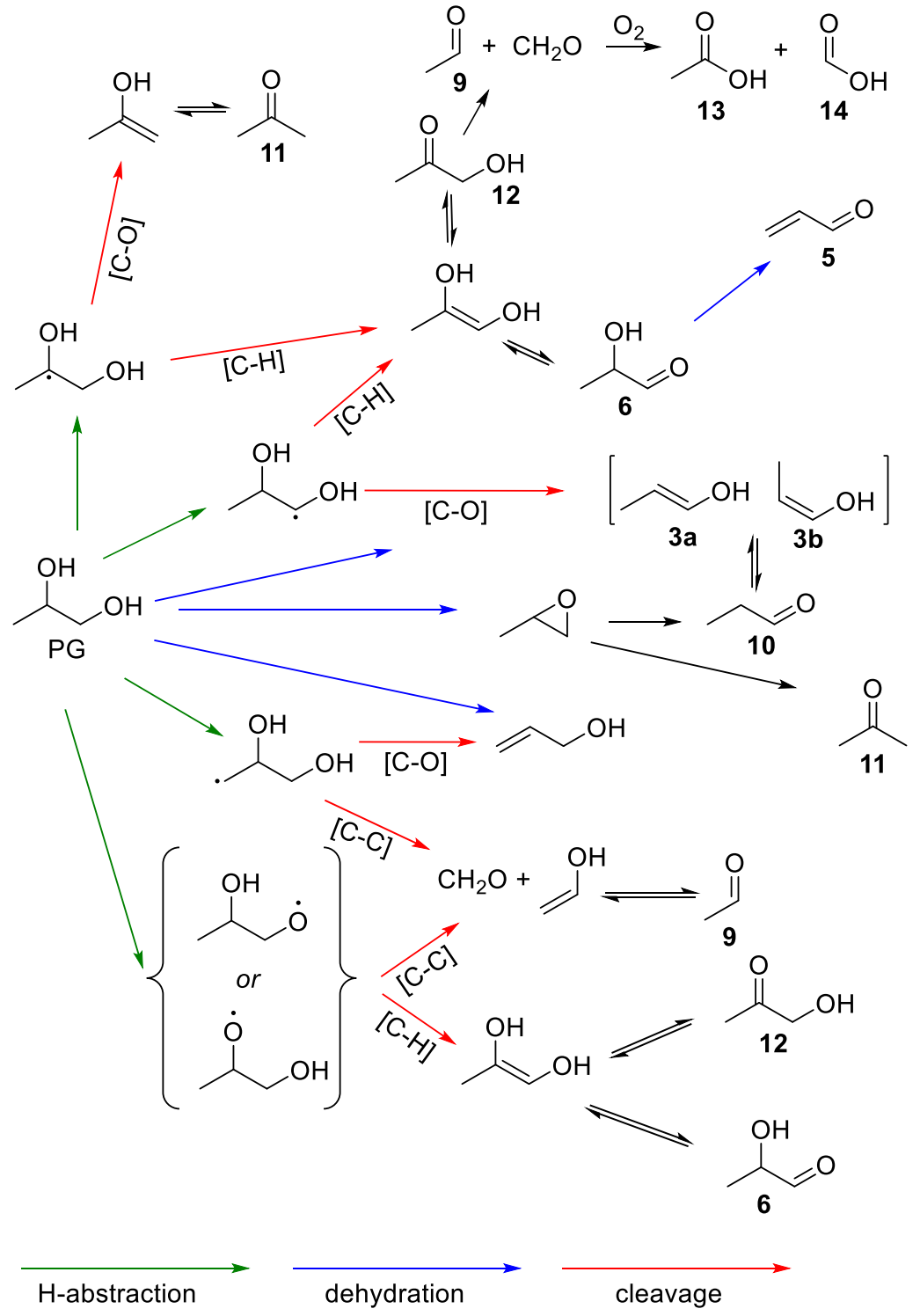

Scheme 1. Thermal degradation pathways for propyene glycol (PG) in the presence of oxygen. Scheme from Jensen et al. (2017). ${ }^{15}$ Labelled compounds shown include: (3a) (Z)-prop-1-en-1-ol, (3b) (E)-prop-1-en-1-ol, (4) dihydroxyacetone, (6) lactaldehyde, (9) acetaldehyde, (10) propanal, (11) acetone, (12) hydroxyacetone (acetol), (13) acetic acid, and (14) formic acid. 


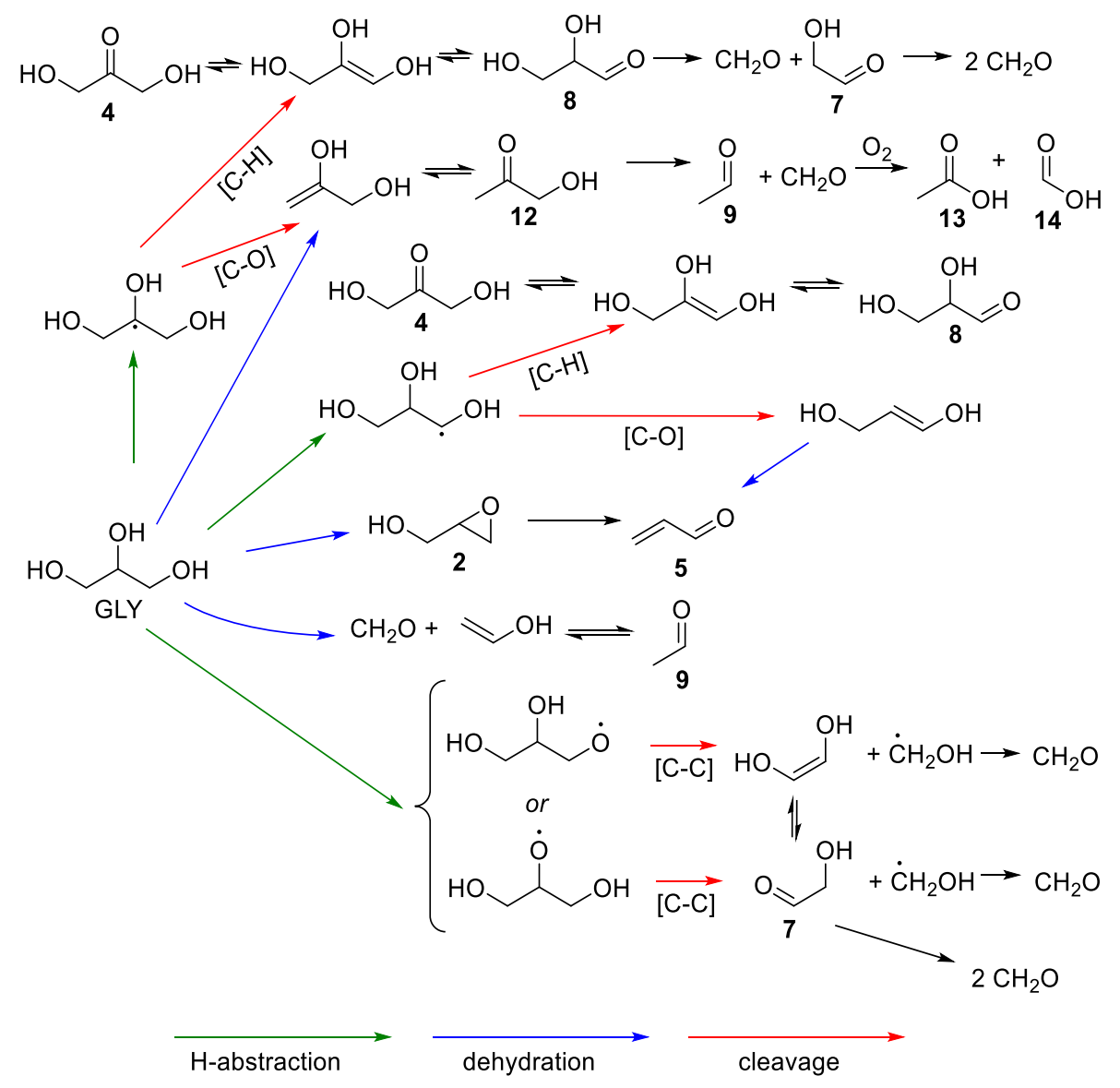

Scheme 2. Thermal degradation pathways for glycerol (GLY) in the presence of oxygen. Scheme from Jensen et al. (2017). ${ }^{15}$ Labelled compounds shown include: (2) glycidol, (4) dihydroxyacetone, (5) acrolein, (7) glycolaldehyde, (8) glyceraldehyde, (9) acetaldehyde, (12) hydroxyacetone (acetol), (13) acetic acid, and (14) formic acid.

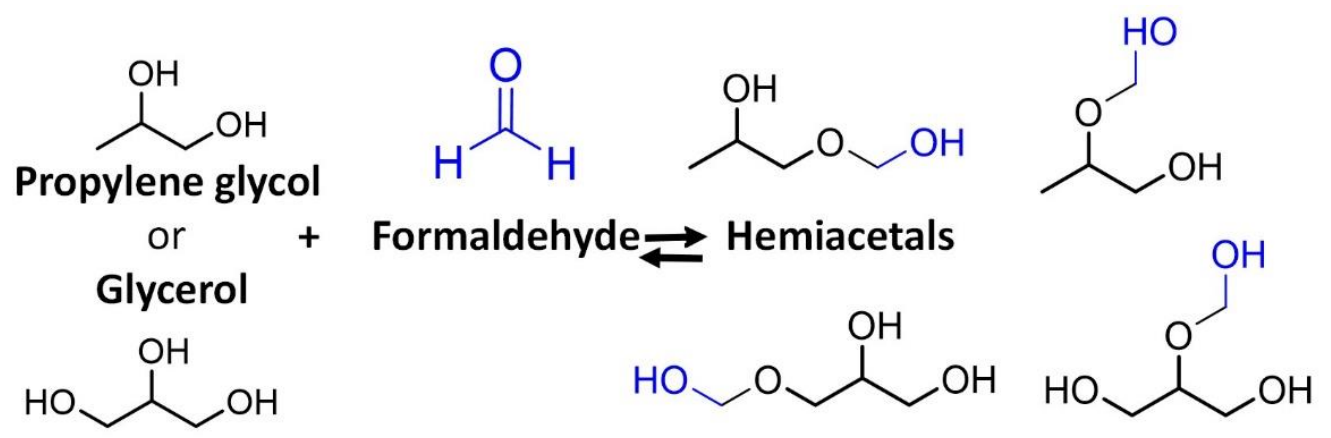

Scheme 3. Formation of hemiacetals from propylene glycol or glycerol reacting with formaldehyde. 
The initial Jensen et al. (2015) study ${ }^{20}$ used an early tank version of an e-cigarette (Innokin iTaste) to study formaldehyde production at two different voltage settings, which were described as "low" (3.3 volts) and "high" (5.0 volts). As expected, more formaldehyde was produced at the higher voltage setting, as was true with the formaldehyde + PG/GL hemiacetals (not detected for the low voltage). This study indicated that even at low settings, some formaldehyde can be produced, that formaldehyde can react with other aerosol products to form other formaldehyde-based molecules, and ultimately that e-cigarettes may not be as safe as some have claimed. ${ }^{23}$ An increased cancer risk due to formaldehyde exposure via vaping was also calculated and reported.

The Jensen et al. (2015) publication was "replicated" by Farsalinos et al. ${ }^{24}$ In the replication study, Farsalinos et al. asserted that formaldehyde was only produced by "dry puffs", that consumers would dislike the taste of a "dry puff", and that consumers would avoid inhaling formaldehyde due to avoidance of an unpleasant taste. To test this, Farsalinos et al. had 26 subjects vape the same model of e-cigarette and e-liquid (Halo brand e-liquid in the "Café Mocha" flavor with $6 \mathrm{mg} / \mathrm{mL}$ nicotine) used by Jensen et al. (2015) and report "dry puffs". The production of formaldehyde was also measured at different voltage settings. This study (by Farsalinos et al.) indicated that $88 \%$ of the tastetesters reported "dry puffs" at settings below or equal to 4.2 volts. Based on this, Farsalinos et al. concluded that 4.0 volts should be the upper limit of use for this ecigarette (and therefore claimed that 5.0 volts was an unrealistic setting used by Jensen et al., 2015). Farsalinos et al. also measured formaldehyde levels at voltages ranging from 
3.3 to 5.0 volts; they reported higher levels of formaldehyde production at both 3.3 and 5.0 volts compared to Jensen et al. (2015), but asserted that 5.0 volts was not a setting that would be used by consumers due to "aversive" taste. Formaldehyde exposure based on their findings was compared with traditional cigarettes and found to be lower, but ignored formaldehyde + PG/GL hemiacetals. The overall conclusion by the authors was that e-cigarettes were "safer" than Jensen et al. (2015) originally reported.

The above Farsalinos et al. (2017) study was followed-up by Salamanca et al. (2018), ${ }^{25}$ again, focusing on formaldehyde levels. Salamanca et al. took issue with the lack of acknowledgment of formaldehyde + PG/GL hemiacetals (Scheme 3) by Farsalinos et al., since only free-formaldehyde levels were reported and are only a part of the total formaldehyde levels produced by an e-cigarette. Other issues with the study conducted by Faralinos et al. were reported by Salamanca et al. For this third study on formaldehyde produced by e-cigarettes, Salamanca et al. used the same device and settings reported by Farsalinos et al. to be non-aversive (4.0 volts/7.3 watts) and evaluated free-formaldehyde and formaldehyde + PG/GL hemiacetals produced. Formaldehyde levels were found to exceed OSHA recommendations using settings that Farsalinos et al. (2017) claimed were non-aversive to the consumer. The results of this study suggest that vapers may not easily detect degradation (due to "aversive" taste) produced by e-cigarettes.

The various formaldehyde levels reported in the above studies highlight another issue in the study of e-cigarettes. A great deal of variation between the degradation levels reported by various laboratories has been found. ${ }^{26}$ To expand on this, Korzun et al. 
reported that different airflow settings through an e-cigarette can result in differences in the resulting degradation profiles, which could contribute to differences between laboratories. ${ }^{27}$ Greater airflow maintains lower coil temperatures, while lower airflow settings can allow the coil to reach higher temperatures. These coil temperature differences are likely what can cause differences in degradation. Korzun et al. specifically report that hydroxyacetone, glycolaldehyde, and formaldehyde are highly moderated by airflow/temperature. ${ }^{27}$ In general, some of the differences between laboratories can be attributed to different puff protocols, sample collection procedures, and instrumental techniques used to analyze degradation. ${ }^{26}$ Devices and settings must be consistent for accurate comparison of degradation levels.

Based on the issues pertaining to formaldehyde and formaldehyde + PG/GL hemiacetal testing for absolute quantities reported in the three studies above (i.e. Jensen et al. (2015), Farsalinos et al. (2017), and Salamanca et al. (2018)), Salamanca et al. (2017) also proposed a method for quantification of these compounds from e-cigarette aerosols. ${ }^{28}$ They also reported that standard DNPH-based collection methods underestimate formaldehyde levels in e-cigarette aerosol due to the formation of other formaldehyde-based complexes. To solve this issue, Salamanca et al. (2017) recommended combining DNPH impingers with cold-traps and then conducting a quantitative NMR (qNMR) analysis to estimate total formaldehyde levels (including formaldehyde + PG/GL hemiacetal products). The exploration/standardization of the best methods for collection and analysis of different types of degradation products is needed in order to promote consistency among e-cigarette degradation findings. 
In addition to degradation products including aldehydes, formaldehyde, and formaldehyde + PG/GL hemiacetals, e-cigarettes can also produce toxins such as benzene when vaped. ${ }^{29}$ Pankow et al. (2017) reported that benzene could be produced from PG/GL, benzoic acid (10 mg/mL in PG/GL), and benzaldehyde (10 mg/mL in PG/GL) during vaping of an e-cigarette. E-liquid tanks evaluated in this study included a Kangertech ${ }^{\mathrm{TM}}$ Protank (1.8 $\Omega$ coil) and a Kangertech ${ }^{\mathrm{TM}}$ Subtank Nano (1.2 $\Omega$ coil), which were both shown to be capable of generating benzene during vaping. Four JUUL ${ }^{\mathrm{TM}}$ flavors were also vaped using a JUUL ${ }^{\mathrm{TM}}$ device, but the resulting aerosols were not found to produce detectable benzene levels by gas chromatography-mass spectrometry.

In general, when evaluating benzene levels generated by operating a Kangertech ${ }^{\mathrm{TM}}$ Protank + a Kangertech ${ }^{\mathrm{TM}}$ EVOD device/battery at 13 watts, more benzene was produced by vaping pure GL rather than pure PG. Furthermore, the amount of benzene increased $\sim 10$ fold when benzoic acid and benzaldehyde were added to the PG/GL mix, respectively. The addition of nicotine to the benzaldehyde + PG/GL mix decreased the amount of benzene produced during vaping by $\sim 1 / 3$. The benzoic acid + PG/GL mix was not as impacted by the addition of nicotine. This study illustrates that different components (i.e. benzaldehyde, benzoic acid) can influence the amount of degradation (e.g. benzene) produced during vaping under identical conditions (same device, settings, etc.).

Dihydroxyacetone is another such toxic degradation product that has been found to be produced by various e-cigarettes. ${ }^{30}$ Vreeke et al. (2017) demonstrated that dihydroxyacetone was produced by all three e-cigarettes tested and that horizontal coils 
(in the following devices: Innokin iTaste VV4 Kanger EVOD and Vaporfi Vox Kanger Protank 2) generally produced more dihydroxyacetone than a vertical coil (Vaporfi Vox Volt). ${ }^{30}$ This was theorized to be related to the temperature of the wet layer (as opposed to the active vaporization layer) of the wicking material due to inefficient wicking, because of device design, including coil orientation (vertical vs horizontal). ${ }^{30}$

Another variable found to enhance degradation produced by e-cigarettes is triacetin. ${ }^{31}$ Vreeke et al. (2018) reported that triacetin (10\% in PG/GL), an e-liquid additive which can function as a carrier solvent, ${ }^{32}$ was capable of increasing degradation when vaped in $\mathrm{PG} / \mathrm{GL} .{ }^{31}$ It was shown that triacetin was hydrolyzed during vaping, producing acetic acid, which then catalyzed degradation of PG/GL. ${ }^{31}$ Degradation components increased by the presence of triacetin included: acetaldehyde, acrolein, and formaldehyde + PG/GL hemiacetals. ${ }^{31}$

\subsection{Fundamental Properties: Boiling Points of E-liquids}

The boiling point of a mixture is the temperature at the liquid-gas phase transition point. Such measurements have practical implications for e-cigarettes, as vaping entails heating the e-liquid via a heating coil until it is capable of vaporization. If the coil temperature is too low, the e-liquid cannot vaporize and the consumer cannot inhale the aerosol. If the temperature is well above the boiling point (i.e. vaporization temperature), then localized heating can occur. This can result in an excess of energy being transferred to the e-liquid, thereby promoting reaction chemistry resulting in degradation of the original e-liquid components. 
As discussed above, degradation products can include aldehydes, cyclic acetals, and chlorpropanols. Compounds generated during vaporization can be problematic for the consumer because it is not necessarily obvious that they are being formed and the quantities are typically unknown. These molecules are not present in e-liquid prior to heating in an e-cigarette, so even if an e-liquid is tested prior to being purchased and the ingredients are listed, the consumer cannot be fully informed of the aerosol contents.

Customizable e-cigarettes allow the consumer to insert coils with a resistance of their choosing, set the wattage/voltage, and sometimes even program the puff profile/temperature/etc. Determining the boiling points of PG+GL mixtures is the first step in determining an appropriate wattage to use for an e-liquid as PG and/or GL are typically the largest components of e-liquids. A boiling point calculation based on this ratio can give a "ballpark" estimate of an appropriate vaporization temperature and was explored herein. Other components such as nicotine, water content, and flavorants can also affect the boiling point/vaporization temperature. The boiling points of PG/GL mixtures with varying ratios and the effect of two concentrations of water ( 2.5 and 5 mol\%) and nicotine (3 mol\%) are explored in this dissertation.

\subsection{Degradation of E-liquids and the Effect of Sucralose}

E-liquids can contain a variety of flavorants and additives. Common flavorants include vanillin, ethyl vanillin, benzaldehyde, and cinnamaldehyde. ${ }^{33}$ Flavors come in broad categories of: sweet, fruity, tobacco, coffee/alcohol and menthol. ${ }^{34}$ None of these products have been approved for use in e-cigarettes nor for inhalation. While dual users (of e-cigarettes + cigarettes) tend to prefer tobacco e-liquid flavors when starting e- 
cigarette use, it has been found that when e-cigarette consumers as a whole are polled, fruity flavors are the most popular among all populations. ${ }^{34}$ The second most popular flavor with youth and young adults is candy/dessert. E-liquids can be purchased in sweet/fruit flavors, and can be modified by the addition of "flavor enhancers". Some of these enhancers include sweeteners such as sucralose, erythritol, ethyl maltol, and others.

Sucralose is one such additive, which is sold both separately and has been identified in a number of commercially available e-liquids. ${ }^{35}$ As a non-nutritive artificial sweetener that has been approved for use in foods, sucralose has not been approved for inhalation or use in e-cigarettes, like most other e-liquid components. ${ }^{36}$ Similar in structure to sucrose, sucralose differs in that three alcohol groups have been replaced with chlorines, one of which has inverted stereochemistry (Figure 3). While sucralose is used in a variety of commercially available foods, it has been demonstrated that sucralose can be unstable when heated. ${ }^{37,38}$

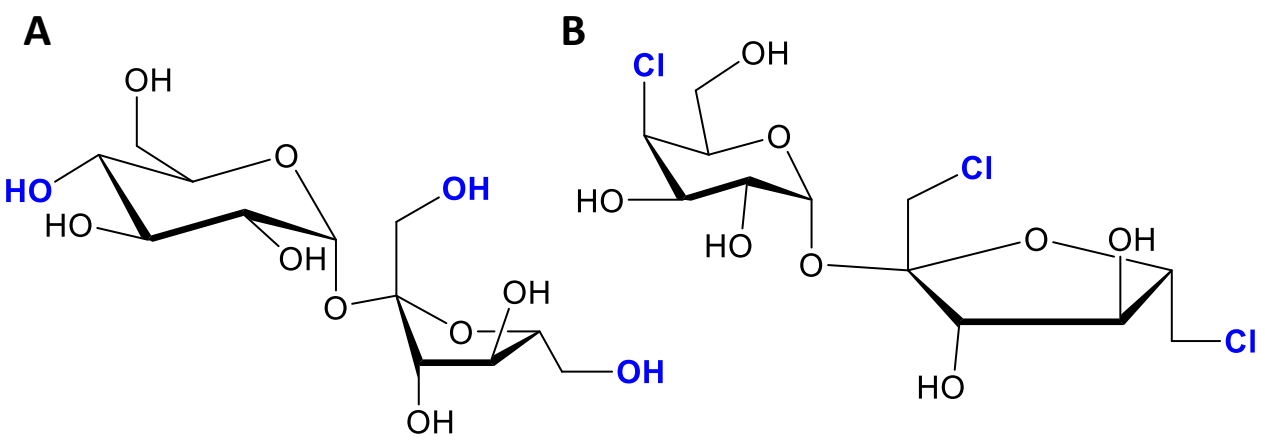

Figure 3. Structures for (A) sucrose versus (B) sucralose.

Sucralose has been shown to be capable of degrading into compounds such as chloropropanols (3-monochloropropanediol, 1,2-dichloropropanol, 1,3- 
dichloropropanol) and furan-based products in the presence of glycerol, when pyrolyzed at $250{ }^{\circ} \mathrm{C} .{ }^{38}$ Furthermore, sucralose can begin to degrade at temperatures below its melting point, producing polychlorinated aromatic hydrocarbons $\sim 98{ }^{\circ} \mathrm{C}$, and decomposing at $\sim 125^{\circ} \mathrm{C} .{ }^{37}$ This temperature is lower than even the boiling point of PG $\left(188.6{ }^{\circ} \mathrm{C}\right)$, indicating that the use of sucralose in e-cigarettes may be problematic due to the temperatures required for volatilization. Furthermore, since sucralose is non-volatile (like sucrose, which degrades when heated), the effect of sucralose on degradation in ecigarettes was of interest.

While PG and GL can degrade when vaped (see above), ${ }^{15}$ the presence of additional components (i.e. flavorants, additives, nicotine, sweeteners, etc.) can enhance or reduce degradation. Since sucralose is non-volatile and has been suggested to be thermally unstable, it was theorized that sucralose could generate additional degradation products and/or enhance existing degradation produced by e-cigarettes. The effect of sucralose on degradation was explored using different concentrations and techniques $\left({ }^{1} \mathrm{H}\right.$ NMR, ion chromatography, gas chromatography-mass spectrometry, and gas chromatography paired with flame ionization detection).

\subsection{Protonation State of Nicotine: Free-base vs. Monoprotonated}

Another important component of many e-liquids is nicotine, the drug of intended exposure for many users. Depending on the $\mathrm{pH}$, nicotine can exist in three different protonation states: free-base (unprotonated), monoprotonated, and diprotonated (Figure 4). Because of the low $\mathrm{pH}$ required to achieve diprotonated nicotine, primarily the first two forms are present in tobacco products. ${ }^{39}$ At $25^{\circ} \mathrm{C}$, the $\mathrm{p} K_{\mathrm{a}}$ values for nicotine are 
3.10 and $8.01 .^{40}$ The different nicotine protonation states partition differently between the gas and particle phases in smoke/aerosol. ${ }^{41}$ Free-base nicotine can exist in both the particulate and gas phases, while monoprotonated nicotine can only exist in the particulate phase ${ }^{41}$ These different phase distributions might result in different pharmacokinetic effects and "impact"/harshness. ${ }^{41}$

Free-base nicotine is considered to have more impact because it can partially exist in the gas phase. ${ }^{42,43}$ As a result, it has been theorized that free-base nicotine can result in faster binding of nicotine to nicotinic acetylcholine receptors in the brain compared to monoprotonated nicotine; this can produce more rapid "satisfaction". Rapid drug absorption and lipid solubility have been suggested to have implications for addiction. ${ }^{44}$

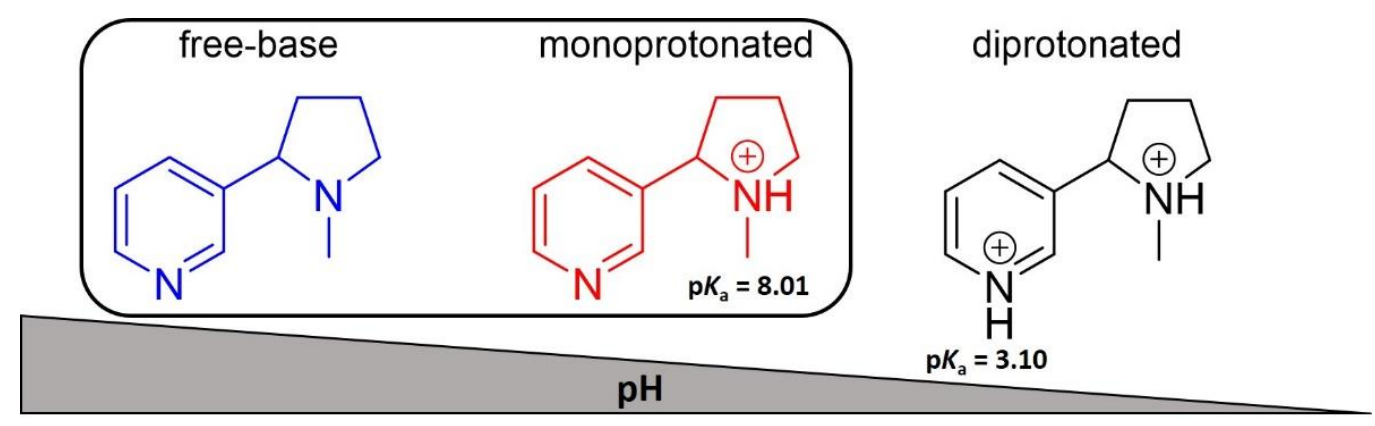

Figure 4. Nicotine can exist in three protonation states depending on the $\mathrm{pH}{ }^{40}$

While satisfaction can be a beneficial component of free-base nicotine for a person using electronic cigarettes to avoid smoking conventional cigarettes, this protonation state may also be harsher than monoprotonated nicotine. The harshness limits the concentration of nicotine that can be inhaled at one time. Monoprotonated nicotine is considered milder than free-base nicotine, and can thus be combined with higher nicotine concentrations, but lacks the impact that free-base nicotine delivers. ${ }^{45}$ These trade-offs 
result in products that, for an optimally addictive/satisfying product, contain both freebase and monoprotonated nicotine.

Commercially available e-liquids vary in both nicotine protonation state and concentration (commonly expressed in $\mathrm{mg} / \mathrm{mL}$ or $\mathrm{wt} \%$ ). The fraction of free-base nicotine, $[\mathrm{Nic}]$, in a product relative to monoprotonated nicotine, $\left[\mathrm{NicH}^{+}\right]$, can be expressed as $\alpha_{\mathrm{fb}}$ (Equation 1). ${ }^{42,43}$

$$
\alpha_{f b} \equiv \frac{[\mathrm{NiC}]}{[\mathrm{Nic}]+\left[\mathrm{NiCH}^{+}\right]}
$$

There is a history of trying to determine the protonation state of nicotine using pH-based methods. ${ }^{45}$ While providing relative observations of free-base to monoprotonated ratios in various products, these methods are flawed for determining absolute ratios because the addition of solvents perturbs the nicotine protonation state equilibrium. ${ }^{41}$ Components of nicotine as well as its protonation state in tobacco products have also been studied using nuclear magnetic resonance (NMR) spectroscopy. ${ }^{46,47}$

NMR spectroscopy is an ideal way to study the protonation state of nicotine as it does not require the addition of solvent to the mixture, thus avoiding perturbation of the system. This is achieved by placing the NMR lock solvent in an outer NMR tube and isolating the sample in an inner NMR tube. ${ }^{48}$ When evaluated at $600 \mathrm{MHz}$ at $40{ }^{\circ} \mathrm{C}$ $\left(\approx 37^{\circ} \mathrm{C}\right)$, the two protonation states of nicotine in most e-liquids (free-base and monoprotonated) result in only one set of nicotine resonances by ${ }^{1} \mathrm{H} \mathrm{NMR}$, rather than two for each protonation state. This is due to coalescence (essentially averaging) of the two sets of nicotine resonances. Coalescence occurs when the exchange rate between the 
two forms (of nicotine in this case) is greater than the chemical shift difference in $\mathrm{Hz}$ between the two forms. ${ }^{46,49}$ As a result, only one averaged set of resonances will appear (Figure 5), even though two similar forms (the two protonation states) exist in the mixture. This is useful, because the chemical shifts of the "averaged" nicotine resonances then can be used to calculate the quantity of nicotine in each protonation state. This is done by first finding the ${ }^{1} \mathrm{H}$ NMR chemical shifts of $100 \%$ free-base nicotine and $100 \%$ monoprotonated nicotine in PG/GL, using the respective reference standards. ${ }^{46}$ When the $\alpha_{\mathrm{fb}}$ value for an e-liquid is desired, the chemical shifts of the nicotine resonances can then be measured and compared with the free-base and monoprotonated nicotine references; $\alpha_{\mathrm{fb}}$ can be determined from the fractional peak shift from the monoprotonated toward the free-base position.

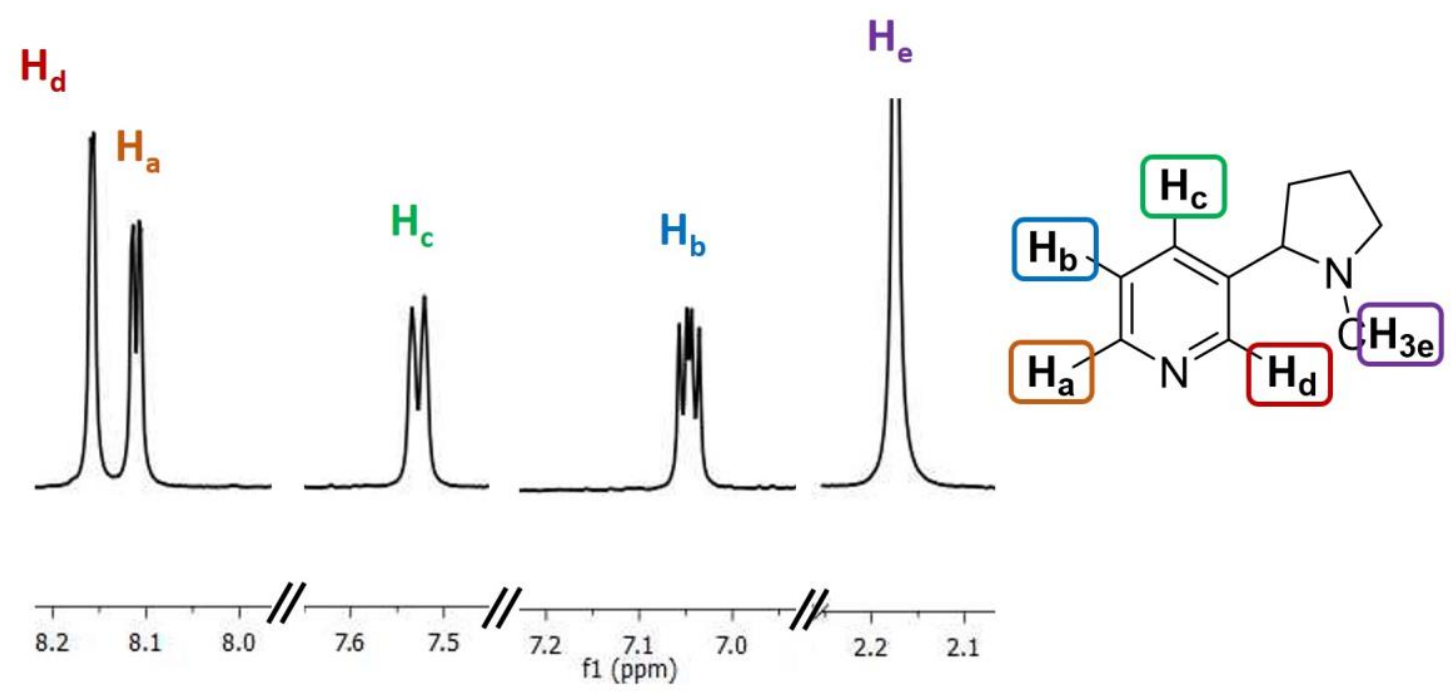

Figure 5. Selected regions of a ${ }^{1} \mathrm{H}$ NMR spectrum showing the nicotine resonances of interest in this work. 
This allows $\alpha_{\mathrm{fb}}$ to be determined for various commercial products. ${ }^{1} \mathrm{H}$ NMR also allows quantitative analysis of the e-liquid components such as determining the PG/GL ratio, concentration of nicotine in an e-liquid, molar ratio of an acid (e.g. benzoic acid, levulinic acid) relative to nicotine, and compound identification. NMR is a versatile technique to study e-liquid as it can provide absolute ratios of components given the proper experimental setup.

I thus used NMR to determine the nicotine content of various commercially available e-liquids (in order to check labelling accuracy), and to determine the $\alpha_{\mathrm{fb}}$ value for each. Nicotine concentration can then be multiplied by $\alpha_{\mathrm{fb}}$ to determine the harshness

of a product. ${ }^{41}$ The underlying assumption was that higher nicotine levels can be tolerated by a consumer with lower $\alpha_{\mathrm{fb}}$. Over time, the protonation state of e-liquids has shifted from having higher $\alpha_{\mathrm{fb}}$ and lower nicotine concentrations (no added acids), to having lower $\alpha_{\mathrm{fb}}$ and higher nicotine concentrations (achieved by the addition of acid(s)). This shift imitates the historical progression over earlier decades for conventional cigarette $\alpha_{\mathrm{fb}}$ content, and could contribute to the addictiveness of the modern e-cigarette.

\subsection{Summary}

The body of work reported in this document centers on e-liquids due to their importance in the chemistry of e-cigarettes, as illustrated by some of the above studies. Of interest, are a) the boiling points of e-liquids (i.e. the temperature at which e-liquids can vaporize in an e-cigarette, thus delivering nicotine and/or flavorants), b) the effect of e-liquid components (e.g. sucralose) on degradation produced by vaping e-liquids in an ecigarette, and c) compositional analysis of commercially available e-liquids (i.e. acids 
components that can change the nicotine protonation). In this document, e-liquid compositional components of interest include the presence and concentration of sucralose, nicotine concentration, nicotine protonation state, and the presence and ratio of acids to nicotine, etc.

In what follows, the majority of the manuscripts discussed herein were conducted using a Kangertech Subbox Mini e-cigarette setup, which includes the KBOX Mini device (+ associated battery), SUBTANK Mini e-liquid tank, and a $1.2 \Omega$ organic cotton coil. This setup was selected as it allows for consistency between studies and eliminates different e-cigarette models as variables. Other devices were used to supplement this work as appropriate. 


\subsection{References}

1 Hon, Lik. "Electronic cigarette." U.S. Patent 8,511,318, issued August 20, 2013. https://patents.google.com/patent/US8511318B2/en [Accessed 13 October 2019]

${ }^{2}$ Giovenco, Daniel P., David Hammond, Catherine G. Corey, Bridget K. Ambrose, and Cristine D. Delnevo. "E-cigarette market trends in traditional US retail channels, 20122013." Nicotine \& Tobacco Research 17, no. 10 (2014): 1279-1283.

${ }^{3}$ King, Brian A., Doris G. Gammon, Kristy L. Marynak, and Todd Rogers. "Electronic cigarette sales in the United States, 2013-2017." JAMA 320, no. 13 (2018): 1379-1380.

${ }^{4}$ Dai, Hongying, and Adam M. Leventhal. "Prevalence of e-Cigarette Use Among Adults in the United States, 2014-2018." JAMA (2019).

${ }^{5}$ Gentzke, Andrea S., MeLisa Creamer, Karen A. Cullen, Bridget K. Ambrose, Gordon Willis, Ahmed Jamal, and Brian A. King. "Vital signs: tobacco product use among middle and high school students - United States, 2011-2018." Morbidity and Mortality Weekly Report 68, no. 6 (2019): 157.

${ }^{6}$ Harrell, Paul Truman, and Thomas Eissenberg. "Automated dripping devices for vapers: RDTAs, bottomfeeders, squonk mods and dripboxes." Tobacco Control 27, no. 4 (2018): 480-482.

${ }^{7}$ Huang, Jidong, Zongshuan Duan, Julian Kwok, Steven Binns, Lisa E. Vera, Yoonsang Kim, Glen Szczypka, and Sherry L. Emery. "Vaping versus JUULing: how the extraordinary growth and marketing of JUUL transformed the US retail e-cigarette market." Tobacco Control 28, no. 2 (2019): 146-151.

${ }^{8}$ Behar, Rachel Z., Wentai Luo, Kevin J. McWhirter, James F. Pankow, and Prue Talbot. "Analytical and toxicological evaluation of flavor chemicals in electronic cigarette refill fluids." Scientific Reports 8, no. 1 (2018): 8288.

9 Tierney, Peyton A., Clarissa D. Karpinski, Jessica E. Brown, Wentai Luo, and James F. Pankow. "Flavour chemicals in electronic cigarette fluids." Tobacco Control 25, no. e1 (2016): e10-e15.

${ }^{10}$ Lisko, Joseph G., Hang Tran, Stephen B. Stanfill, Benjamin C. Blount, and Clifford H. Watson. "Chemical composition and evaluation of nicotine, tobacco alkaloids, $\mathrm{pH}$, and selected flavors in e-cigarette cartridges and refill solutions." Nicotine \& Tobacco Research 17, no. 10 (2015): 1270-1278. 
${ }^{11}$ Aszyk, Justyna, Paweł Kubica, Mateusz Kacper Woźniak, Jacek Namieśnik, Andrzej Wasik, and Agata Kot-Wasik. "Evaluation of flavour profiles in e-cigarette refill solutions using gas chromatography-tandem mass spectrometry." Journal of Chromatography A 1547 (2018): 86-98.

${ }^{12}$ Kubica, Paweł, Agata Kot-Wasik, Andrzej Wasik, and Jacek Namieśnik. "“Dilute \& shoot" approach for rapid determination of trace amounts of nicotine in zero-level eliquids by reversed phase liquid chromatography and hydrophilic interactions liquid chromatography coupled with tandem mass spectrometry-electrospray ionization." Journal of Chromatography A 1289 (2013): 13-18.

${ }^{13}$ Goniewicz, Maciej Lukasz, Jakub Knysak, Michal Gawron, Leon Kosmider, Andrzej Sobczak, Jolanta Kurek, Adam Prokopowicz et al. "Levels of selected carcinogens and toxicants in vapour from electronic cigarettes." Tobacco Control 23, no. 2 (2014): 133139.

${ }^{14}$ Strongin, Robert M. "E-Cigarette chemistry and analytical detection." Annual Review of Analytical Chemistry 12 (2019).

${ }^{15}$ Jensen, R. P., Strongin, R. M., \& Peyton, D. H. (2017). Solvent chemistry in the electronic cigarette reaction vessel. Scientific Reports, 7, 42549.

${ }^{16}$ Zhao, Jiayuan, Yipei Zhang, Jennifer D. Sisler, Justine Shaffer, Stephen S. Leonard, Anna M. Morris, Yong Qian, Dhimiter Bello, and Philip Demokritou. "Assessment of reactive oxygen species generated by electronic cigarettes using acellular and cellular approaches." Journal of Hazardous Materials 344 (2018): 549-557.

${ }^{17}$ Soussy, Sarah, EL-Hellani Ahmad, Rima Baalbaki, Rola Salman, Alan Shihadeh, and Najat A. Saliba. "Detection of 5-hydroxymethylfurfural and furfural in the aerosol of electronic cigarettes." Tobacco Control 25, no. Suppl 2 (2016): ii88-ii93.

${ }^{18}$ Hess, Catherine Ann, Pablo Olmedo, Ana Navas-Acien, Walter Goessler, Joanna E. Cohen, and Ana Maria Rule. "E-cigarettes as a source of toxic and potentially carcinogenic metals." Environmental Research 152 (2017): 221-225.

${ }^{19}$ Khlystov, Andrey, and Vera Samburova. "Flavoring compounds dominate toxic aldehyde production during e-cigarette vaping." Environmental Science \& Technology 50, no. 23 (2016): 13080-13085.

${ }^{20}$ Jensen, R. Paul, Wentai Luo, James F. Pankow, Robert M. Strongin, and David H. Peyton. "Hidden formaldehyde in e-cigarette aerosols." New England Journal of Medicine 372, no. 4 (2015): 392-394. 
${ }^{21}$ Hutzler, Christoph, Meike Paschke, Svetlana Kruschinski, Frank Henkler, Jürgen Hahn, and Andreas Luch. "Chemical hazards present in liquids and vapors of electronic cigarettes." Archives of Toxicology 88, no. 7 (2014): 1295-1308.

${ }^{22}$ Bekki, Kanae, Shigehisa Uchiyama, Kazushi Ohta, Yohei Inaba, Hideki Nakagome, and Naoki Kunugita. "Carbonyl compounds generated from electronic cigarettes." International Journal of Environmental Research and Public Health 11, no. 11 (2014): 11192-11200.

${ }^{23}$ Glantz, Stanton A., and David W. Bareham. "E-cigarettes: use, effects on smoking, risks, and policy implications." Annual Review of Public Health 39 (2018): 215-235.

${ }^{24}$ Farsalinos, Konstantinos E., Vassilis Voudris, Alketa Spyrou, and Konstantinos Poulas. "E-cigarettes emit very high formaldehyde levels only in conditions that are aversive to users: a replication study under verified realistic use conditions." Food and Chemical Toxicology 109 (2017): 90-94.

${ }^{25}$ Salamanca, James C., Jiries Meehan-Atrash, Shawna Vreeke, Jorge O. Escobedo, David H. Peyton, and Robert M. Strongin. "E-cigarettes can emit formaldehyde at high levels under conditions that have been reported to be non-averse to users." Scientific Reports 8, no. 1 (2018): 7559.

${ }^{26}$ Strongin, Robert M. "E-Cigarette chemistry and analytical detection." Annual Review of Analytical Chemistry 12 (2019).

${ }^{27}$ Korzun, Tetiana, Maryana Lazurko, Ian Munhenzva, Kelley C. Barsanti, Yilin Huang, R. Paul Jensen, Jorge O. Escobedo, Wentai Luo, David H. Peyton, and Robert M. Strongin. "E-cigarette airflow rate modulates toxicant profiles and can lead to concerning levels of solvent consumption." ACS Omega 3, no. 1 (2018): 30-36.

${ }^{28}$ Salamanca, James C., Ian Munhenzva, Jorge O. Escobedo, R. Paul Jensen, Angela Shaw, Robert Campbell, Wentai Luo, David H. Peyton, and Robert M. Strongin. "Formaldehyde hemiacetal sampling, recovery, and quantification from electronic cigarette aerosols." Scientific Reports 7, no. 1 (2017): 11044.

${ }^{29}$ Pankow, James F., Kilsun Kim, Kevin J. McWhirter, Wentai Luo, Jorge O. Escobedo, Robert M. Strongin, Anna K. Duell, and David H. Peyton. "Benzene formation in electronic cigarettes." PloS One 12, no. 3 (2017): e0173055.

${ }^{30}$ Vreeke, Shawna, Tetiana Korzun, Wentai Luo, R. Paul Jensen, David H. Peyton, and Robert M. Strongin. "Dihydroxyacetone levels in electronic cigarettes: Wick temperature and toxin formation." Aerosol Science and Technology 52, no. 4 (2018): 370-376. 
${ }^{31}$ Vreeke, Shawna, David H. Peyton, and Robert M. Strongin. "Triacetin enhances levels of acrolein, formaldehyde hemiacetals, and acetaldehyde in electronic cigarette aerosols." ACS Omega 3, no. 7 (2018): 7165-7170.

${ }^{32}$ Fiume, M. Z. "Final report on the safety assessment of triacetin." International Journal of Toxicology 22 (2003): 1-10.

${ }^{33}$ Tierney, Peyton A., Clarissa D. Karpinski, Jessica E. Brown, Wentai Luo, and James F. Pankow. "Flavour chemicals in electronic cigarette fluids." Tobacco Control 25, no. e1 (2016): e10-e15.

${ }^{34}$ Harrell, Melissa B., Scott R. Weaver, Alexandra Loukas, M. Creamer, C. N. Marti, C. D. Jackson, J. W. Heath et al. "Flavored e-cigarette use: Characterizing youth, young adult, and adult users." Preventive Medicine Reports 5 (2017): 33-40.

${ }^{35}$ Duell, Anna K., Kevin J. McWhirter, Tetiana Korzun, Robert M. Strongin, and David H. Peyton. "Sucralose-enhanced degradation of electronic cigarette liquids during vaping." Chemical Research in Toxicology 32, no. 6 (2019): 1241-1249.

${ }^{36}$ Grotz, V. Lee, and Ian C. Munro. "An overview of the safety of sucralose." Regulatory Toxicology and Pharmacology 55, no. 1 (2009): 1-5.

${ }^{37}$ De Oliveira, Diogo N., Maico De Menezes, and Rodrigo R. Catharino. "Thermal degradation of sucralose: a combination of analytical methods to determine stability and chlorinated byproducts." Scientific Reports 5 (2015): 9598.

${ }^{38}$ Rahn, Anja, and Varoujan A. Yaylayan. "Thermal degradation of sucralose and its potential in generating chloropropanols in the presence of glycerol." Food Chemistry 118, no. 1 (2010): 56-61.

${ }^{39}$ Proctor, Robert N., and Robert Proctor. Golden holocaust: origins of the cigarette catastrophe and the case for abolition. Univ of California Press, 2011.

${ }^{40}$ Barlow, R. B., and J. T. Hamilton. "Effects of $\mathrm{pH}$ on the activity of nicotine and nicotine monomethiodide on the rat diaphragm preparation." British Journal of Pharmacology and Chemotherapy 18, no. 3 (1962): 543.

${ }^{41}$ Pankow, James F. "A consideration of the role of gas/particle partitioning in the deposition of nicotine and other tobacco smoke compounds in the respiratory tract." Chemical Research in Toxicology 14, no. 11 (2001): 1465-1481.

${ }^{42}$ Pankow, James F., Brian T. Mader, Lorne M. Isabelle, Wentai Luo, Andrea Pavlick, and Cikui Liang. "Conversion of nicotine in tobacco smoke to its volatile and available free-base form through the action of gaseous ammonia." Environmental Science \& Technology 31, no. 8 (1997): 2428-2433. 
${ }^{43}$ Pankow, James F., Ameer D. Tavakoli, Wentai Luo, and Lorne M. Isabelle. "Percent free base nicotine in the tobacco smoke particulate matter of selected commercial and reference cigarettes." Chemical Research in Toxicology 16, no. 8 (2003): 1014-1018.

${ }^{44}$ Farre, Magi, and Jordi Camí. "Pharmacokinetic considerations in abuse liability evaluation." British Journal of Addiction 86, no. 12 (1991): 1601-1606.

${ }^{45}$ Wayne, G. Ferris, G. N. Connolly, and J. E. Henningfield. "Brand differences of freebase nicotine delivery in cigarette smoke: the view of the tobacco industry documents." Tobacco Control 15, no. 3 (2006): 189-198.

${ }^{46}$ Pankow, James F., Kelley C. Barsanti, and David H. Peyton. "Fraction of free-base nicotine in fresh smoke particulate matter from the Eclipse "cigarette" by ${ }^{1} \mathrm{H}$ NMR spectroscopy." Chemical Research in Toxicology 16, no. 1 (2003): 23-27.

${ }^{47}$ Barsanti, Kelley C., Wentai Luo, Lorne M. Isabelle, James F. Pankow, and David H. Peyton. "Tobacco smoke particulate matter chemistry by NMR." Magnetic Resonance in Chemistry 45, no. 2 (2007): 167-170.

${ }^{48}$ Duell, Anna K., James F. Pankow, and David H. Peyton. "Free-base nicotine determination in electronic cigarette liquids by ${ }^{1} \mathrm{H}$ NMR spectroscopy." Chemical Research in Toxicology 31, no. 6 (2018): 431-434.

${ }^{49}$ Bryant, Robert G. "The NMR time scale." Journal of Chemical Education 60, no. 11 (1983): 933. 
2. Boiling Points of the Propylene Glycol + Glycerol System at 1 Atmosphere Pressure: 188.6-292 ${ }^{\circ} \mathrm{C}$ Without and With Added Water or Nicotine

Anna K. Duell ${ }^{\mathrm{a}}$, James F. Pankow ${ }^{\mathrm{a}, \mathrm{b}}$, Samantha M. Gillette ${ }^{\mathrm{a}}$, and David H. Peyton ${ }^{\mathrm{a}}$

${ }^{a}$ Department of Chemistry, Portland State University, Portland, OR, USA

${ }^{b}$ Department of Civil and Environmental Engineering, Portland State University,

Portland, OR, USA

This manuscript has been published and is adapted from:

Anna K. Duell, James F. Pankow, Samantha M. Gillette \& David H. Peyton (2018)

Boiling points of the propylene glycol + glycerol system at 1 atmosphere pressure: 188.6$292^{\circ} \mathrm{C}$ without and with added water or nicotine, Chemical Engineering

Communications, 205:12, 1691-1700, DOI: 10.1080/00986445.2018.1468758

Link to source: https://www.tandfonline.com/doi/abs/10.1080/00986445.2018.1468758 


\subsection{Abstract}

In electronic cigarettes ("electronic nicotine delivery systems", ENDS), mixtures of propylene glycol (PG) and/or glycerol (GL; aka "vegetable glycerin", VG) with nicotine are vaporized to create a nicotine-containing aerosol. For a given composition, the temperature required to boil the liquid at 1 atmosphere must be at least somewhat greater than the boiling point (BP). The use of ENDS is increasing rapidly worldwide, yet the BP characteristics of the PG+GL system have been characterized as the mixtures; here we re-do this, but significantly, also study the effects of added water and nicotine. BP values at 1 atmosphere pressure were measured over the full binary composition range. Fits based on the Gibbs-Konovalov theorem provide BP as a function of composition (by mole-percent, by weight-percent, and by volume-percent). BPs of PG+GL mixtures were then tested in the presence of additives such as water (2.5 and 5 mol\% added) and nicotine (3 mol\%). Water was found to decrease the BP of PG+GL mixtures significantly at all compositions tested, and nicotine was found to decrease the BP of PG+GL mixtures containing 75 GL: 25PG (by moles) or more. The effect of added water $(5,10$, and $15 \mathrm{~mol} \%$ added $)$ on electronic cigarette degradation production (some aldehydes and formaldehyde hemiacetals) was examined and found to have no significant impact on solvent (PG or GL) degradation for the particular device used.

Keywords: E-Cigarette liquid; Electronic cigarette; Electronic nicotine delivery system (ENDS); Glycerol; Heat exchanger fluid; Propylene glycol 


\subsection{Introduction}

Propylene glycol (PG) and glycerol (GL) are high production volume (HPV) chemicals used in numerous industrial and consumer applications (Pendergrass, 1999; Teschke et al., 2005). First, they serve as heat exchanging fluids in solar hot water and geothermal energy systems, including as PG+GL mixtures. Second, they are main ingredients in the nicotine-containing liquids (e-liquids) used in electronic cigarettes (aka "e-cigarettes", "electronic nicotine delivery systems", "ENDSs") either individually or as a mixture. The dependence of boiling point (BP) temperature on composition is of interest in heat exchangers, e-cigarette applications, and for separations by distillation (Chen et al., 2015). Boiling is very unwelcome in heat exchange applications, but essential in the e-cigarettes (boiling must occur if the desired subsequent condensation aerosol is to form (Zhang et al., 2013; Glycerine as a heat transfer fluid and antifreeze, 2016). Globally, from 2014 to 2015, solar hot water capacity grew $6.4 \%$ from 409 to 435 gigawatts (Renewables 2016 Global status report, 2016; Mickle, 2015). For the ecigarette industry, global growth was 58\% in 2014 (Market Research on Vapour Devices, 2016). The number of regular adult e-cigarette users in the US in 2014 has been estimated at 11.8 million, with the number of "ever-users" estimated at 40.2 million (Schoenborn and Gindi, 2015).

Remarkably, BP behavior in the binary PG+GL system has received little direct study (Talih et al., 2017). For heat exchange applications, such information is needed during design to avoid vapor formation, and in e-cigarette applications, the information reveals the minimum temperatures that the ingredient chemicals (which may include 
flavor chemicals) will experience. Also, it is now well known that heating of "e-liquids" can lead to degradation products, some of which are toxic (formaldehyde (Jensen et al., 2015), acrolein and other aldehydes, and aromatic hydrocarbons (Hahn et al., 2014)). In a mixture, boiling occurs when the vapor pressure contributions from all components combine to become at least somewhat greater than the system pressure. It is thus useful to know how BP varies with composition in the PG+GL system.

Antoine equation parameterizations for the temperature-dependent vapor pressures of pure PG $\left(p^{\mathrm{o}} \mathrm{PG}\right)$ and pure GL ( $\left.p^{\mathrm{o}}{ }_{\mathrm{GL}}\right)$ are available (Table 1). By assuming ideal liquid mixtures (i.e., applicability of Raoult's Law), one can use these parameterizations to predict the $\mathrm{BP}$ values for the full range of compositions for $\mathrm{PG}+\mathrm{GL}$ mixtures according to

$$
p_{\mathrm{TOT}}=x_{\mathrm{PG}} p_{\mathrm{PG}}^{\mathrm{o}}+x_{\mathrm{GL}} p_{\mathrm{GL}}^{\mathrm{o}}
$$

For each value of $\mathrm{x}_{\mathrm{PG}}$ (with $\mathrm{x}_{\mathrm{GL}}=1-\mathrm{x}_{\mathrm{PG}}$ ), Equation (1) can be solved to obtain the normal $\mathrm{BP}$ as the value of $\mathrm{T}$ that gives $\mathrm{p}_{\text {тот }}=1 \mathrm{~atm}$. The predicted $\mathrm{BP}$ values thereby obtained in Table 2 are largely within the reported applicability range for the Antoine fit for GL, but are above the applicability range for PG: the applicability range for PG only extends to the BP of PG, which is below the BP for every mixture of PG and GL. The goal of this work was to carry out BP measurements for the full range of PG and GL mixtures, but also extending this by adding the effect of added water and nicotine. 
Table 1. Antoine Equation Parameters for Vapor Pressure $p^{\circ}$ (bar) of the Pure Liquids Propylene Glycol (PG) and Glycerol (GL), with Applicable Temperature Ranges, $\log _{10}\left(p^{o}\right)=A-B /(T(K)+C)$. (1 atm $=1.01325$ bar. $)$ http://webbook.nist.gov/chemistry/

\begin{tabular}{llllccl}
\hline & $\underline{\boldsymbol{t}\left({ }^{\circ} \mathrm{C}\right) \text { range }}$ & $\underline{\boldsymbol{T}(\mathrm{K}) \text { range }}$ & $\underline{\boldsymbol{A}}$ & $\underline{\boldsymbol{B}}$ & $\underline{\boldsymbol{C}}$ & Reference \\
PG & $45.6-188.3$ & $318.7-461.4$ & 6.07936 & 2692.2 & -17.94 & Richardson, 1886 \\
GL & $183.3-260.5$ & $456.4-533.6$ & 3.9374 & 1411.5 & -200.566 & Stull, 1947
\end{tabular}


Table 2. Boiling Point Values at 1 Atm Pressure Assuming Raoult's Law (Ideal Mixtures). $\quad p_{\mathrm{TOT}}(T)=x_{\mathrm{PG}} p_{\mathrm{PG}}^{\mathrm{o}}+x_{\mathrm{GL}} p_{\mathrm{GL}}^{\mathrm{o}}=1.01325 \mathrm{bar}(=1 \mathrm{~atm})$ For Antoine Equation Parameters in Table 1.

\begin{tabular}{llll} 
& & & Boiling Point \\
$\underline{X_{\mathrm{PG}}}$ & $\underline{x_{\mathrm{GL}}}$ & $t\left({ }^{\circ} \mathrm{C}\right)$ & $T(\mathrm{~K})$ \\
\hline 1.00 & 0.00 & 188.0 & 461.2 \\
0.95 & 0.05 & 189.6 & 462.8 \\
0.90 & 0.10 & 191.3 & 464.4 \\
0.85 & 0.15 & 193.1 & 466.2 \\
0.80 & 0.20 & 194.9 & 468.1 \\
0.75 & 0.25 & 196.9 & 470.1 \\
0.70 & 0.30 & 199.1 & 472.2 \\
0.65 & 0.35 & 201.4 & 474.6 \\
0.60 & 0.40 & 203.9 & 477.1 \\
0.55 & 0.45 & 206.7 & 479.8 \\
0.50 & 0.50 & 209.6 & 482.8 \\
0.45 & 0.55 & 212.9 & 486.1 \\
0.40 & 0.60 & 216.6 & 489.8 \\
0.35 & 0.65 & 220.7 & 493.9 \\
0.30 & 0.70 & 225.4 & 498.6 \\
0.25 & 0.75 & 230.9 & 504.0 \\
0.20 & 0.80 & 237.3 & 510.4 \\
0.15 & 0.85 & 245.1 & 518.2 \\
0.10 & 0.90 & 254.8 & 528.0 \\
0.05 & 0.95 & 540.9 \\
0.00 & 1.00 & 559.6 \\
\hline
\end{tabular}


2.3. Materials and Methods

2.3.1. Materials

\subsubsection{Boiling Point and ${ }^{1} H$ NMR Testing}

United States Pharmacopeia grade PG and GL were obtained from Sigma-Aldrich (St. Louis, MO). Upon each opening and resealing, caps were wrapped with paraffin film to reduce hygroscopic absorption of water from the atmosphere. Reagents were $99.9+\%$ pure, which was verified by nuclear magnetic resonance spectroscopy (NMR). (S)(-)nicotine (99\%) was acquired from Alfa Aesar (Haverhill, MA). Deuterium oxide (99.9\% $\left.{ }^{2} \mathrm{H}, 0.1 \%{ }^{1} \mathrm{H}\right)$ and dimethyl sulfoxide- $d_{6}\left(99.9 \%{ }^{2} \mathrm{H}, 0.1 \%{ }^{1} \mathrm{H}\right)$ were from Cambridge Isotope Laboratories (Tewksbury, MA), respectively.

Disposable capillary tubes $(25 \mu \mathrm{L})$ were obtained from Drummond Scientific (Broomall, PA). Outer boiling point capillaries and an MP80 Melting Point System were obtained from Mettler Toledo (Columbus, OH). Graphite carbon powder (“'-20 + 60 mesh") was from Alfa Products (Danvers, MA).

\subsubsection{2. $\quad$ Vaporized E-liquid Collection}

An NE-1660 model syringe pump (New Era Pump Systems Incorporated; Farmingdale, NY) was equipped with Monoject ${ }^{\mathrm{TM}} 140 \mathrm{~mL}$ syringes (Covidien; Dublin, Ireland), a valve control box (New Era Pump Systems Incorporated), and a 3-port solenoid valve (12 VDC; Humphrey; Kalamazoo, MI) with barbed brass fittings (McMaster-Carr; Elmhurst, IL) in order to collect vaporized e-liquid samples. Nylon Luer lock fittings (Cole-Parmer; Vernon Hills, IL) were used to connect silicone rubber laboratory tubing (Cole-Parmer; 0.125” I.D., 0.250" O.D., wall thickness 0.0625”) to BD 
(Franklin Lakes, NJ) PrecisionGlide ${ }^{\mathrm{TM}}$ Needles (18 gauge, $1.2 \mathrm{~mm}$ X 25mm), which entered and exited the $2 \mathrm{~mL}$ sample collection vials (screw thread autosampler vials; Fisher Scientific; Pittsburgh, PA). Vials were equipped with $8 \mathrm{~mm}$ screw thread autosampler rubber septum caps (Fisher Scientific). A KangerTech KBOX Mini (Shenzhen KangerTech Technology Co., Ltd; Guangdong, China) was equipped with a 18650 Sony (Tokyo, Japan) 2100 mAh High Discharge Flat Top battery, KangerTech Subtank Mini tank, and KangerTech 1.2 X OCC (“Organic Cotton Coil”). A custom stainless-steel mouthpiece was developed to securely connect the mouth of the e-cigarette to the tubing.

\subsubsection{Sample Preparation}

\subsubsection{Large-scale Boiling Point Determinations}

Mixtures of PG and GL were prepared in triplicate at room temperature using 40 $\mathrm{mL}$ brown glass vials. The mixtures ranged from 0 to $100 \%$ by mass $\mathrm{GL}$ in increments of $10 \%$ by weight, for a total of 33 individually prepared $\sim 20 \mathrm{~mL}$ samples.

\subsubsection{Micro-scale Boiling Point Determinations}

Mixtures of PG and GL were prepared in batch sizes ranging from 5 to $10 \mathrm{~mL}$ in $40 \mathrm{~mL}$ brown glass vials. Five sets of mixtures were prepared. Each set of mixtures ranged from 0 to $100 \%$ by mols GL in increments of $25 \%$ by mol. To each set, water or nicotine was added to prepare one of the following: $2.5 \mathrm{~mol} \%$ water, $5 \mathrm{~mol} \%$ water, or 3 mol\% nicotine (equivalent to $64.2-64.3 \mathrm{mg} / \mathrm{mL}$ nicotine, depending on whether pure PG or GL is used), for a total of 25 individually prepared mixtures. 


\subsubsection{All Boiling Point Samples}

Mass fractions were used as the basis of the preparations rather than volume fractions because of greater ease, with viscous liquids, in measuring mass versus volume amounts delivered. Vial caps were wrapped with paraffin film. Each sample was mixed by shaking for five minutes then stored in the dark for no more than $24 \mathrm{~h}$ before testing.

\subsubsection{E-liquids for Vaporization}

PG and GL were combined in equal molar quantities and prepared gravimetrically to produce a stock mixture ("no added water"; $\sim 125 \mathrm{~g}, \sim 109 \mathrm{~mL}$ ) for vaporization experiments. After this PG:GL stock was confirmed to be approximately equimolar by ${ }^{1} \mathrm{H}$ NMR, three additional mixtures were prepared by taking aliquots of the stock mixture (by mass; $22 \mathrm{~g} /$ sample) and adding deionized distilled water (by volume) to produce 5, 10, and $15 \mathrm{~mol} \%$-added water samples. These prepared e-liquids were evaluated by NMR and found to contain approximately $0,7,11$, and 14 mol\% water. Samples were stored in brown bottles, wrapped with paraffin film to reduce hygroscopic water absorption from the air, and used for experimentation within $8 \mathrm{~h}$ of preparation.

\subsubsection{Boiling Point Determinations}

\subsubsection{Large-scale}

Prior to heating, a "pre-boiling" $10 \mu \mathrm{L}$ aliquot of each sample was mixed with $600 \mu \mathrm{L} \mathrm{D}_{2} \mathrm{O}$ for analysis by NMR. The BP of the remaining $\sim 20 \mathrm{~mL}$ of sample was determined using the apparatus represented in Figure 1. A three-necked round bottom flask was fitted with two reflux condensers that allowed nitrogen gas $\left(\mathrm{N}_{2}\right)$ to enter the boiling chamber, then exit via an oil bubbler (not shown); this permitted $\mathrm{N}_{2}$ gas to flow 
freely through the system while maintaining an anoxic environment at ambient pressure. An HH12B digital thermometer and a KTSS-HH temperature probe from Omega (Stamford, CT) were fitted in the third flask opening.

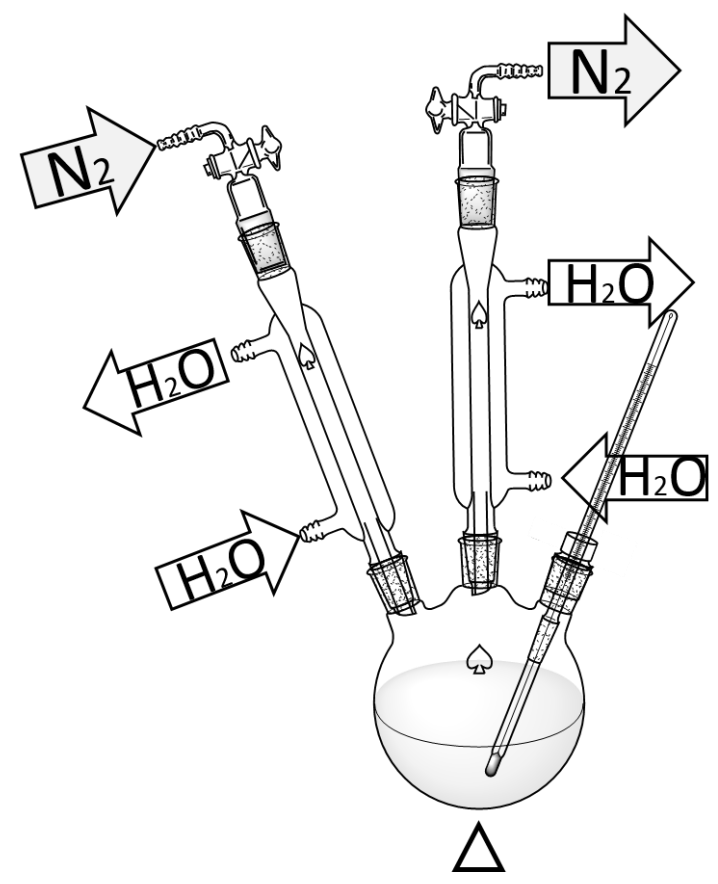

Figure 1. Schematic of Boiling Point (BP) Setup. Thermometer in third port represents a digital thermometer.

The digital temperature probe accuracy was reported by the manufacturer as $\leq 1.3^{\circ} \mathrm{C}$, and this was verified by measuring the BP values of three liquids at 1atm pressure: water (BP: $100.0^{\circ} \mathrm{C}$ ), acetophenone (BP: $204^{\circ} \mathrm{C}$ ), and ethyl benzoate (BP: $\left.214^{\circ} \mathrm{C}\right)$. Standard deviation (SD) values for the triplicate PG and GL mixtures were found to be at most 0.5 ${ }^{\circ} \mathrm{C}$, which is smaller than the probe accuracy (as reported by the manufacturer). Below $200{ }^{\circ} \mathrm{C}$, the probe displayed four significant figures, including one decimal; above 200 
${ }^{\circ} \mathrm{C}$, only three significant figures were displayed. The flask was held in a rheostatregulated heating mantle; mixing was provided using a stir plate. The system was thoroughly flushed with $\mathrm{N}_{2}$ gas prior to heating. Samples were gradually heated over 30 90 min while stirring continuously until boiling was observed. Boiling temperatures were determined as the stable temperature at which each sample exhibited a steady rolling boil for at least five minutes. Each system was then allowed to return to room temperature under $\mathrm{N}_{2}$ gas. A "post-boiling" aliquot $(10 \mu \mathrm{L})$ was taken for analysis by NMR. The preand post-boiling NMR samples were collected to ensure that the boiling process did not considerably alter composition.

\subsubsection{Micro-scale}

The Mettler-Toledo MP80 Melting Point System was used to conduct all microscale boiling point trials. The system was operated using the manufacturer's instructions, with some minor modifications that were required due to the high mixture viscosities. Each external boiling point capillary tube was loaded with $100-200 \mu \mathrm{L}$ of sample, $\sim 1 \mathrm{mg}$ of graphite carbon powder, and a disposable $25 \mu \mathrm{L}$ capillary tube. The $25 \mu \mathrm{L}$ capillary tube was fractured at the sample end prior to insertion in the outer capillary tube in order to serve as a surface for bubble nucleation.

Boiling point trials were conducted in triplicate. Preliminary trials were conducted prior to trials in order to determine an appropriate temperature range to test for each sample. The temperature range tested for each sample (ending temperature - starting temperature) varied and was as small as $25^{\circ}$ and as large as $55^{\circ}$, and was centered around a potential boiling point. Variation in temperature range size was due to the inconsistent 
behavior of mixtures. The brightness was set to $40 \%$ and samples were heated at a rate of $10^{\circ} \mathrm{C} / \mathrm{min}$ within the selected range.

The boiling point of each sample was initially determined two different ways. (1) During a boiling point trial, the MP80 system attempted to report the boiling point by measuring the bubble rate as recorded by the instrument; once the bubble rate surpassed a certain threshold, the instrument reported the current temperature as the boiling point ("instrument-determined"). (2) The instrument recorded a video of the boiling point trial, which was magnified and viewed on the instrument screen during the trial, as well as reviewed after each trial ("visual determination"). Because of the viscous nature of the mixtures, precise "instrument-determined" boiling points were not reliable; while "visual determination" was concluded to be the most reliable and reproducible method of the two boiling point measurement methods, and so was used to determine the boiling points reported herein.

\subsection{4. $\quad 1 \mathrm{D}{ }^{1} \mathrm{H}$ NMR Analyses}

\subsubsection{Large-scale Boiling Point Samples}

The NMR analyses conducted on each pre- and post-boiling $10 \mu \mathrm{L}$ aliquot (as diluted in $600 \mu \mathrm{L}$ of $\mathrm{D}_{2} \mathrm{O}$ ) were carried out using a Bruker (Billerica, MA) Avance III spectrometer $(599.90 \mathrm{MHz})$ with a $5 \mathrm{~mm}$ TXI probe. A pulse sequence of zg30 was used to acquire the data, with the relaxation delay value (d1) set to $5 \mathrm{~s}$, in combination with the $30^{\circ}$ observation pulse of the $\mathrm{zg} 30$ experiment to allow for full relaxation, and so give reliable integrations. All NMR spectra were processed using the software package MestReNova 9.0 (Santiago de Compostela, Spain; Mnova, 2016). Spectra were auto- 
phase corrected (but with manual adjustment as needed), followed by auto-baseline correction. Integral values were verified by manually correcting some spectra; the results of which were found to agree with the values from the corresponding auto-corrected spectrum values, to within $0.5 \%$. Satellite peaks caused by the $1.1 \%$ natural abundance of

${ }^{13} \mathrm{C}$ overlapped with some peaks of interest, thereby potentially introducing uncertainty to the mole ratio calculation. This uncertainty was minimized by integrating peaks of interest and adjusting for the natural abundance of ${ }^{13} \mathrm{C}$, allowing for more accurate measurements of mole ratios. This produced mole ratio measurements that were within $1.4 \mathrm{~mol} \%$ of values based on the mass preparation method. The difference between the NMR-determined and predicted mol\% GL (based on initial masses) was calculated; the absolute values of the differences were averaged for all trials to determine the average difference $( \pm \mathrm{SD})$, which is $0.3 \pm 0.3 \mathrm{~mol} \%$ GL. Despite gravimetric sample preparation, NMR was used to assess post-boiling composition for analysis because these results were most closely associated in time with observed boiling.

\subsubsection{Micro-scale Boiling Point Samples}

Each stock mixture was tested by ${ }^{1} \mathrm{H}$ NMR as in section Large-scale boiling point samples (above), within $1 \mathrm{~h}$ of BP evaluation, by combining $100 \mu \mathrm{L}$ of sample with 400 $\mu \mathrm{L}$ of DMSO- $d_{6}$ and mixing. In this case, the relaxation delay (D1) was set to $3 \mathrm{~s}$, because this value was determined to be sufficient for accurate integration of the peaks resulting from these mixtures. Despite gravimetric sample preparation, NMR was used to assess and report composition due to the presence of water in all samples and the hygroscopic nature of PG and GL. 


\subsubsection{Vaporized E-liquid Samples}

Samples were produced by vaporizing e-liquid and having the vapor pass through a $2 \mathrm{~mL}$ autosampler vial containing $500 \mu \mathrm{L}$ of DMSO- $d_{6}$. The resulting samplecontaining DMSO- $d_{6}$ was transferred to a NMR tube and tested by ${ }^{1} \mathrm{H}$ NMR methods described in section Large-scale boiling point samples (above) but with a relaxation delay (D1) of $3 \mathrm{~s}$. Samples were tested within $24 \mathrm{~h}$ of being produced.

\subsubsection{Vaporized E-liquid Sample Collection: the Effect of Added Water}

\subsubsection{E-cigarette Preparation}

The method for e-cigarette vaping and NMR sample collection was adapted from Jensen et al. (2017). A KangerTech KBOX Mini equipped with a 2100 mAh battery was fully charged prior to sample generation. KangerTech $1.2 \Omega$ organic cotton coils were inserted into three KangerTech Subtank Minis. Coils used in this experiment were "conditioned" by vaporizing 10-20 puffs (see section E-cigarette "puff" protocol for puff methods) of PG + GL e-liquid (50 mol\% of each) at $26 \mathrm{~W}$ and were used to generate samples at $24 \mathrm{~W}$ or lower to determine if they resulted in e-liquid degradation. Only coils that showed significant e-liquid degradation, as indicated by the production of aldehydes and formaldehyde hemiacetals, were chosen for this experiment. We hypothesized that since the addition of water ( 2.5 and $5 \mathrm{~mol} \%$ ) to PG + GL mixtures decreased the BP, then the vaporized e-liquids containing more water could result in less degradation due to the lower vaporization temperature required. Since we hypothesized that more water in the eliquid would produce less degradation, we wanted to ensure that significant degradation could be seen prior to the addition of more water. For the "no added water" condition, 
tanks were filled with 4.6-5.1 g of e-liquid and the coil was thoroughly wetted with eliquid. After samples were collected for this condition, tanks were drained of e-liquid and dried with lint-free tissues. Tanks were then rinsed with $\sim 1 \mathrm{~mL}$ of the next refill liquid, which was discarded, and then tanks were refilled for testing with the same e-liquid condition. This process was repeated for each testing condition for each tank. For the 5, 10, and $15 \mathrm{~mol} \%$ added water conditions, 4.3-4.7 g, 4.0-4.6 g, and 4.1-4.4 g of e-liquid was added to tanks, respectively.

\subsubsection{2. $\quad$ E-cigarette Vapor Collection Setup}

The charged KangerTech KBOX Mini, attached to the e-liquid-filled Subtank Mini, was vertically positioned and a custom-made stainless-steel mouthpiece was inserted into the tank opening using a rubber O-ring to provide a seal. A nylon Luer lock fitting was screwed into the stainless-steel mouthpiece connecting it to $\sim 9 \mathrm{~cm}$ of silicone tubing. At the other end of the tubing, was a barbed Luer lock fitting attached to an 18 gauge needle. The tubing and needle arced $\sim 180^{\circ}$ from the mouthpiece of the e-cigarette. This first needle was inserted fully through the rubber septum of the cap on the $2 \mathrm{~mL}$ sample vial (containing $500 \mu \mathrm{L}$ DMSO-d6), such that the tip of the needle was touching the side of the vial above the solvent line. A second (exit) needle was also inserted through the same rubber septum, but just far enough that the needle opening was inside the vial. This exit needle was also connected to a Luer lock fitting that securely attached to a second piece of silicone tubing $(\sim 9 \mathrm{~cm})$. This final piece of tubing connected the sample collection system to a 3-port solenoid value. The solenoid value allowed the pump to withdraw the sample from the e-cigarette through one opening during the 
withdrawal time-period, and eject the remaining puff into the fume hood. The final opening on the solenoid valve connected with tubing to the sample collection system to a $140 \mathrm{~mL}$ syringe (controlled by the pump). The solenoid valve was controlled by the valve control box, which was wired to the pump. The pump was programmed according to section, E-cigarette "puff” protocol (below).

\subsubsection{E-cigarette "Puff" Protocol}

Samples were generated using the puffing parameters set forth by CORESTA (Cooperation Centre for Scientific Research Relative to Tobacco) for e-cigarette aerosol sample production (CORESTA, 2015). The puff duration was $3 \mathrm{~s}$, the puff volume was $55 \mathrm{~mL}$, and the puff frequency was 2 puffs/ minute (one puff started every 30s). The power button on the e-cigarette was activated one second prior to the start of sample collection (syringe withdrawal), and held for a total of $4 \mathrm{~s}$ (including the $3 \mathrm{~s}$ puff). The interpuff interval (distance between the end of one puff and the start of the next) was thus $27 \mathrm{~s}$.

\subsubsection{E-cigarette Vapor Collection at $22 \mathrm{~W}$}

The coils used for this experiment were previously conditioned and analyzed by NMR in terms of degradation production (section E-cigarette preparation). The same KangerTech KBOX Mini and battery were used for all samples and was set to $22 \mathrm{~W}$ (these coils are rated for up to $26 \mathrm{~W}$ ); the device was charged between conditions. Tanks and vials were weighed before and after sample collection so that the fraction of aerosol that was trapped in the sample vial (\%-trapped) could be calculated for each sample. The mouthpiece of each tank was cleaned after the generation of each sample prior to 
weighing. After tanks were filled with new e-liquids, 10 "wicking puffs" were collected into a vial and discarded. Approximately 1-5 min elapsed between the end of the final wicking puff and the collection of a sample collected for NMR (three puffs); one of each was collected from each tank per water condition, for a total of 24 samples.

\subsection{Results and Discussion}

\subsubsection{Large-scale Boiling Point Determinations}

The overall precision of the large-scale BP determination technique was established for each method by calculating the standard deviation of triplicate boiling point $\left({ }^{\circ} \mathrm{C}\right.$ ) values (Table 3). Standard deviation in terms of \% GL for each large-scale method resulted in $\leq 0.2 \%$ for $w t \%$ GL (based on initial mixture masses), $\leq 0.4 \%$ for the mol $\%$, and $\leq 0.5 \%$ for the vol $\%$ (calculated using the mol $\%$ determined by NMR). The similarities between the volume $\%$ and mol $\%$ at each temperature are simply a consequence of the density/molecular mass ratio being nearly the same for both PG and GL.

$\mathrm{BP}$ values of $\mathrm{PG}$ and GL mixtures $\left(\mathrm{t}_{\mathrm{b}},{ }^{\circ} \mathrm{C}\right)$ shown in Figure 2 as $\mathrm{BP}$ versus mol percent were fit with a Gibbs-Konovalov parameterization (Malesinski, 1965; Al-Jiboury, 2007).

$$
t_{\mathrm{b}}\left({ }^{\circ} C\right)=x_{\mathrm{PG}} t_{\mathrm{b}, \mathrm{PG}}+x_{\mathrm{GL}} t_{\mathrm{b}, \mathrm{GL}}+x_{\mathrm{PG}} x_{\mathrm{GL}}\left(A+B\left(x_{\mathrm{PG}}-x_{\mathrm{GL}}\right)-C\left(x_{\mathrm{PG}}-x_{\mathrm{GL}}\right)^{2}+D\left(x_{\mathrm{PG}}-x_{\mathrm{GL}}\right)^{3}\right)
$$

where $t_{b, P G}$ and $t_{b, G L}\left({ }^{\circ} \mathrm{C}\right)$ are the measured boiling points of pure PG and GL, respectively. Fit values of the coefficients A, B, C, and D for Equation (2) (i.e., using mol fraction composition) were found by minimizing the sum of the residuals using the 
Microsoft Excel Solver (Frontline Systems Inc., Incline Village, NV) add-in. Fit values were similarly obtained using volume and weight fraction values. Coefficients A-D are presented in Table 4. Corresponding calculated BP values are given in Table 5. GibbsKonovalov calculated values (Table 5) were compared with the Antoine equation values (Table 2) and found to differ by up to $6.7^{\circ} \mathrm{C}$ at the upper range (beginning $>230{ }^{\circ} \mathrm{C}$ ), with an average difference $( \pm \mathrm{SD})$ of $1.8 \pm 1.9^{\circ} \mathrm{C}$ over the entire range (Table S3).

Table 3. Measured Boiling Point (BP) Values of Propylene Glycol (PG) and Glycerol (GL) Mixtures With Volume \%, Weight \%, and Mol \% (N=3).

\% Glycerol (Average \pm 1 SD)

\begin{tabular}{c|ccc}
$\begin{array}{c}\text { BP average } \\
\text { SD }\left({ }^{\circ} \mathrm{C}\right)\end{array}$ & volume \% & weight $\%$ & mol \% \\
$\mathbf{1 8 8 . 6} \pm \mathbf{0 . 6}$ & $0 \pm 0$ & $0.0 \pm 0.0$ & $0.0 \pm 0$ \\
$\mathbf{1 9 1 . 6} \pm \mathbf{0 . 2}$ & $8.3 \pm 0.1$ & $10.0 \pm 0.0$ & $8.3 \pm 0.1$ \\
$\mathbf{1 9 4 . 7} \pm \mathbf{0 . 4}$ & $17.3 \pm 0.2$ & $20.1 \pm 0.0$ & $17.3 \pm 0.2$ \\
$\mathbf{1 9 8 . 6} \pm \mathbf{0 . 2}$ & $26.1 \pm 0.2$ & $30.0 \pm 0.0$ & $26.2 \pm 0.2$ \\
$\mathbf{2 0 3} \pm \mathbf{0 . 0}$ & $35.8 \pm 0.2$ & $40.1 \pm 0.1$ & $35.8 \pm 0.2$ \\
$\mathbf{2 0 8} \pm \mathbf{0 . 6}$ & $45.5 \pm 0.1$ & $49.9 \pm 0.0$ & $45.6 \pm 0.1$ \\
$\mathbf{2 1 4} \pm \mathbf{0 . 0}$ & $55.8 \pm 0.4$ & $60.0 \pm 0.0$ & $55.9 \pm 0.4$ \\
$\mathbf{2 2 3} \pm \mathbf{0 . 6}$ & $66.4 \pm 0.4$ & $69.9 \pm 0.0$ & $66.4 \pm 0.4$ \\
$\mathbf{2 3 6} \pm \mathbf{0 . 0}$ & $77.6 \pm 0.5$ & $80.0 \pm 0.0$ & $77.6 \pm 0.4$ \\
$\mathbf{2 5 8} \pm \mathbf{0 . 6}$ & $89.2 \pm 0.2$ & $90.0 \pm 0.2$ & $89.2 \pm 0.2$ \\
$\mathbf{2 9 2} \pm \mathbf{0 . 0}$ & $99.9 \pm 0.1$ & $100.0 \pm 0.0$ & $99.9 \pm 0.1$
\end{tabular}




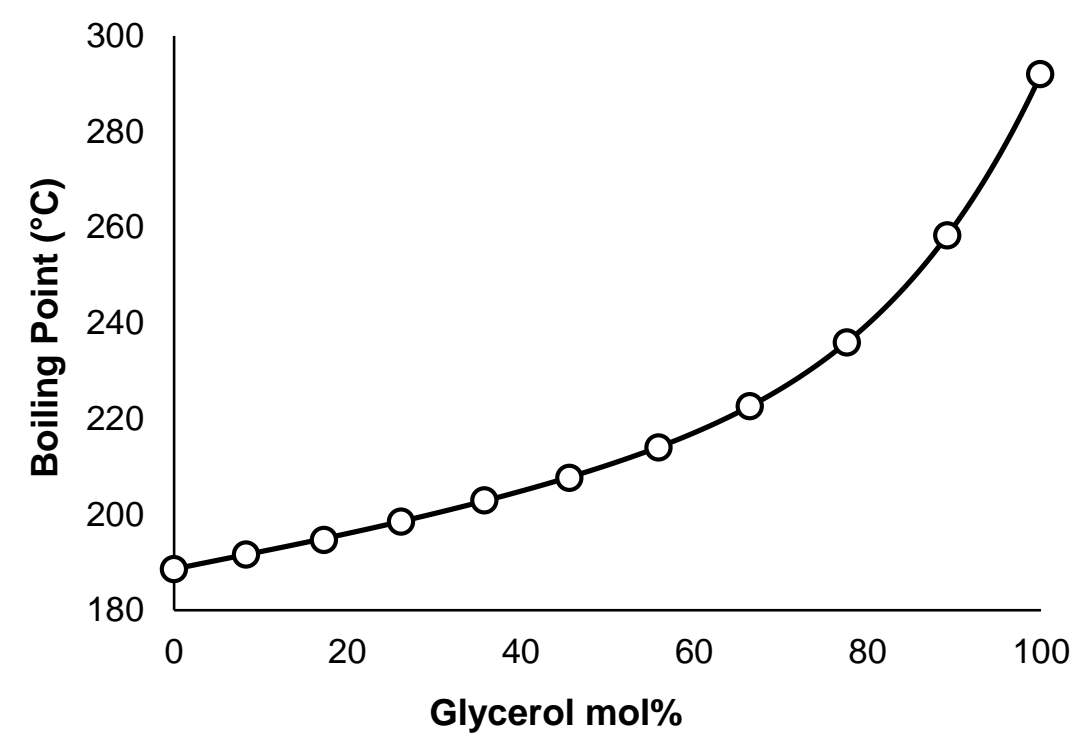

Figure 2. Averages of Triplicate Boiling Point Measurements for Mixtures Composed of Propylene Glycol (PG) and Glycerol (GL). Mole percent GL post-boiling was determined by NMR analysis. Error bars as \pm 1 SD are too small to be seen; the largest SD is 0.6. 
Table 4. Coefficients Determined for Gibbs-Konovalov Parameterization of Propylene Glycol (PG) and Glycerol (GL) Boiling Point Data.

\begin{tabular}{ccccc} 
& \multicolumn{4}{c}{ coefficient } \\
& $\boldsymbol{A}$ & $\boldsymbol{B}$ & $\boldsymbol{C}$ & $\boldsymbol{D}$ \\
\cline { 2 - 5 } vol \% & -119.9 & -87.3 & 55.8 & -22.6 \\
wt \% & -130.3 & -100.8 & 66.9 & -10.6 \\
mol \% & -119.9 & -87.3 & 55.9 & -22.5
\end{tabular}

Table 5. Calculated Boiling Point (BP) Values $\left({ }^{\circ} \mathrm{C}\right)$ for Propylene Glycol and Glycerol (GL) Mixtures by Volume, Weight, and Mole Percent GL using Coefficients A-D in Table 4, and Equation 2.

\begin{tabular}{|c|c|c|c|c|c|c|c|c|c|c|c|c|}
\hline$\%$ GL & 0 & 5 & 10 & 15 & 20 & 25 & 30 & 35 & 40 & 45 & 50 & $\% G L$ \\
\hline & 189 & 190 & 192 & 194 & 196 & 198 & 200 & 202 & 205 & 207 & 210 & vol \% \\
\hline & 189 & 190 & 191 & 193 & 195 & 197 & 199 & 201 & 203 & 205 & 208 & $w t \%$ \\
\hline 2 & 189 & 190 & 192 & 194 & 196 & 198 & 200 & 202 & 205 & 207 & 210 & $\mathrm{~mol} \%$ \\
\hline$\%$ GL & 55 & 60 & 65 & 70 & 75 & 80 & 85 & 90 & 95 & 100 & & $\% G L$ \\
\hline & 213 & 217 & 221 & 226 & 232 & 240 & 249 & 260 & 274 & 292 & & vol \% \\
\hline O & 211 & 214 & 218 & 223 & 229 & 237 & 246 & 258 & 273 & 292 & & $w t \%$ \\
\hline 0 & 213 & 217 & 221 & 226 & 232 & 240 & 249 & 260 & 274 & 292 & & mol \% \\
\hline
\end{tabular}




\subsubsection{Micro-scale Boiling Point Trials}

Large-scale boiling was possible and reliable for PG+GL mixtures, but additives such as water or nicotine made such determinations difficult. Determining the BPs for PG+GL mixtures with additives was made possible using the micro-scale BP method. Mixtures of PG+GL were tested using the micro-scale method $(0,25,50,75$, and 100 $\mathrm{mol} \% \mathrm{GL}$ ) and found to agree with the previously determined/calculated BPs (Table 5) within $3^{\circ}$. The absolute differences between the large and micro-scale BPs (data not shown) were averaged and found to be $1.5 \pm 1.0{ }^{\circ} \mathrm{C}(\mathrm{SD})$.

Samples analyzed using the micro-scale boiling point system were tested by NMR to verify composition because PG and GL are hygroscopic. NMR results were used to ensure that similar amounts of water were present in samples within each condition. Although gravimetric methods were used to prepare samples, NMR composition results were used to determine the mole \%GL in relation to only PG for comparison with BPs. NMR composition results produced mole ratio measurements that were within $3.1 \mathrm{~mol} \%$ of values based on the mass preparation method. The largest uncertainty, which was associated with the $3 \mathrm{~mol} \%$ nicotine sample in 75 GL: 25PG (by mol\%), was attributed to the mol\% water, and is due to the hygroscopic nature of the mixture. All other values differed from the expected composition by less than $2.1 \mathrm{~mol} \%$. The absolute difference between the actual (NMR-determined) and predicted mol\% GL (based on gravimetric preparation of the samples) was calculated for each sample; the average ( \pm SD) of the absolute difference for each component was $0.7 \pm 0.7 \mathrm{~mol} \%$ for GL, $0.9 \pm 0.6 \mathrm{~mol} \%$ for 
PG, $1.4 \pm 0.7 \mathrm{~mol} \%$ for water, and $0.1 \pm 0.1 \mathrm{~mol} \%$ for nicotine. Water was the largest source of uncertainty for all samples.

An additional source of uncertainty arises from the water content of the samples, as it was difficult to calculate using NMR integrations due to overlap between the water peak and resonances from PG and GL. In most samples, the water peak was located under the PG and/or GL peaks, and so was determined by peak subtraction. Despite careful phasing, baseline correction, and processing, this could contribute to the uncertainty in the determination of the water content in mixtures by NMR. However, since samples within a batch were prepared at the same time and based on mass data, they are fairly similar in terms of content. The average added water content determined by mass for the $5 \mathrm{~mol} \%$ water samples was $4.9 \pm 0.2 \mathrm{~mol} \%$ and $2.6 \pm 0.2 \mathrm{~mol} \%$ water for the " $2.5 \mathrm{~mol} \%$ added water samples". By NMR, the average water content in the " 5 mol\% added water" samples was $5.6 \pm 1.6(\mathrm{SD})$, and $3.9 \pm 0.6$ for " $2.5 \mathrm{~mol} \%$ added water" samples. The average nicotine content in the $3 \mathrm{~mol} \%$ nicotine mixtures was found to be $2.9 \pm 0.2 \mathrm{~mol} \%$ (as calculated from gravimetric data) and $2.7 \pm 0.2 \mathrm{~mol} \%$ by NMR.

The boiling points of $\mathrm{PG}+\mathrm{GL}$ mixtures with additives (Table 6) indicate that water at molar concentrations of 2.5 and $5 \mathrm{~mol} \%$ of the total mixture decrease the BP of PG+GL mixtures ranging from $0 \%$ to $100 \%$ GL. Nicotine (3 mol $\%$ of the total mixture) was found to decrease the BP, but the only significant changes involved 75 GL: 25PG and 100 GL: OPG (by mol\%). Water as an additive altered the BP of PG+GL mixtures by up to $\sim 60^{\circ}$ for $5 \mathrm{~mol} \%$ water (in $100 \%$ GL), and up to $\sim 30^{\circ}$ for $2.5 \mathrm{~mol} \%$ water (in $100 \%$ GL); see Figure 3. Nicotine (3 mol\%) lowered the BP by up to $15^{\circ}$ (100\% GL), but 
lowered the BP for 0 GL: 100PG to $50 \mathrm{GL}: 50$ PG (by mol\%) by less than one degree.

One mol\% added nicotine was also evaluated, and the BP values were found not to be significantly perturbed across the entire range of PG+GL mixtures (data not shown).

Table 6. Micro-Scale Boiling Points (BP) of Propylene Glycol (PG) + Glycerol (GL) Mixtures with Additives in Relation to Mol\% GL (Relative to Moles PG Only).

\begin{tabular}{rl}
5 mol\% water \\
\cline { 1 - 1 } mol\% GL & BP $\left({ }^{\circ} \mathbf{C}\right)$ \\
100 & $230.7 \pm 0.6$ \\
75 & $203.3 \pm 2.5$ \\
50 & $191.7 \pm 1.5$ \\
26 & $185 \pm 0.0$ \\
0 & $173.3 \pm 1.2$
\end{tabular}

2.5 mol\% water

mol\% GL BP ( $\left.{ }^{\circ} \mathrm{C}\right)$

$100261 \pm 1.0$

$76 \quad 212.5 \pm 0.9$

$50 \quad 199.7 \pm 0.2$

$26191.0 \pm 0.9$

$0 \quad 183.3 \pm 1.2$
3 mol\% nicotine

mol\% GL BP ( $\left.{ }^{\circ} \mathrm{C}\right)$

$100 \quad 277 \pm 0.0$

$76 \quad 229.5 \pm 1.3$

$50 \quad 210.1 \pm 0.4$

$26198.3 \pm 0.6$

$0 \quad 188.2 \pm 0.3$ 


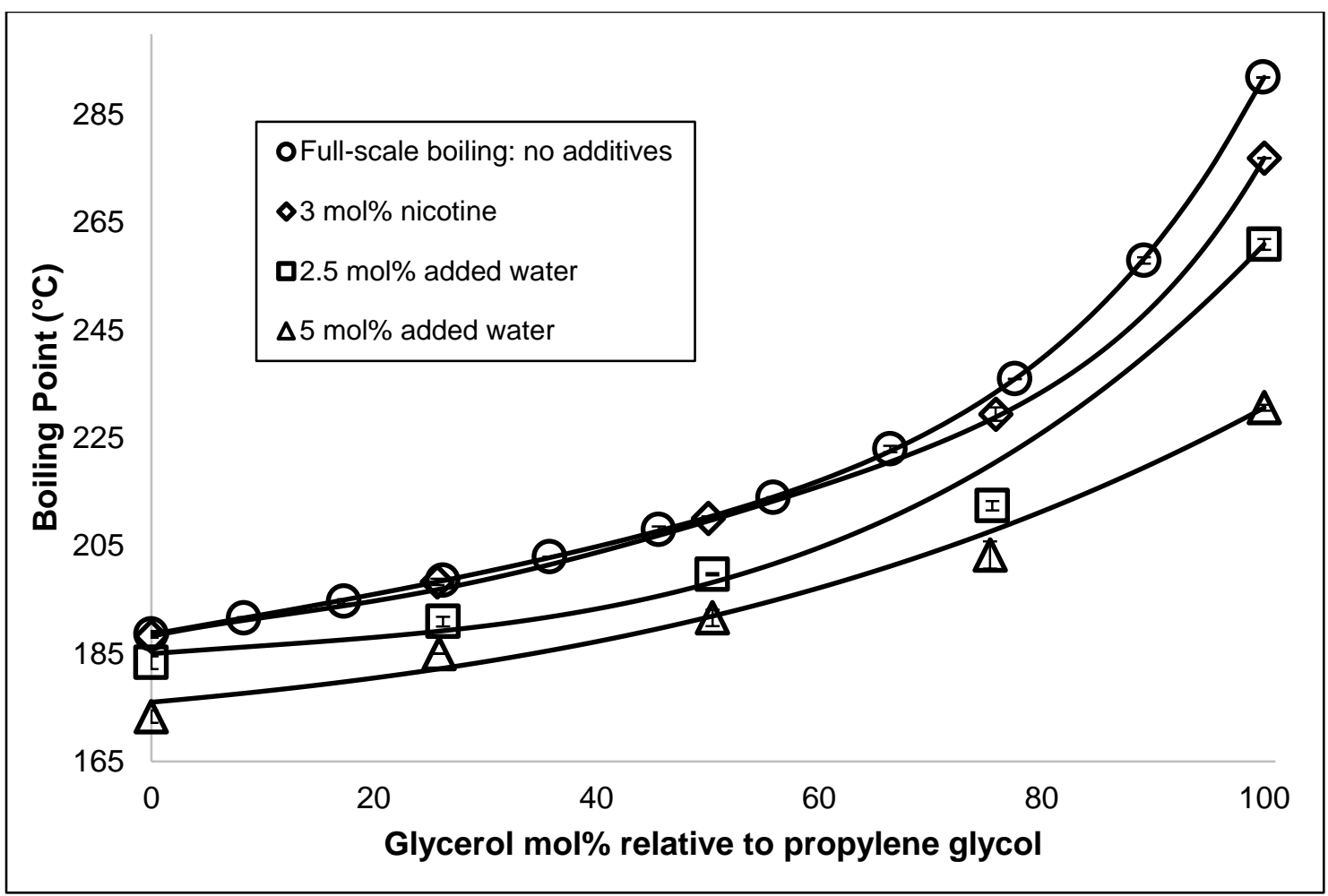

Figure 3. Averages of Triplicate Boiling Point Measurements for Mixtures Composed of Propylene Glycol (PG) and Glycerol (GL). The boiling points of propylene glycol + glycerol mixtures were determined in the absence of additives ("Large-scale boiling: no additives"), as well as in the presence of $3 \mathrm{~mol} \%$ nicotine, $2.5 \mathrm{~mol} \%$ added water, and 5 mol $\%$ added water. Fits are included to guide the eye, rather than allow extrapolated boiling points. Error $( \pm 1 \mathrm{SD})$ is provided for all data points $(\mathrm{N}=3)$. 
${ }^{1} \mathrm{H}$ NMR spectroscopy has been shown to be useful for evaluating PG+GL eliquid degradation during e-cigarette vaping (Jensen et al., 2015, 2017). Samples vaporized using a KangerTech KBOX Mini in combination with a KangerTech Subtank Mini were analyzed by NMR and degradation was examined as a function of added water. Degradation was considered by examining aldehyde peaks (propanal, acetaldehyde, glyceraldehyde, glycolaldehyde, lactaldehyde, and acrolein) and PG and/or GL formaldehyde hemiacetal peaks and were identified based on chemical shifts and splitting patterns (Jensen et al., 2017). Based on the micro-scale BP trials (section Microscale boiling point trials), the " 2.5 " and " 5 mol\% added water" conditions would lower the BPs by $\sim 10^{\circ}$ and $\sim 20^{\circ}$, respectively. We hypothesized that lowering the BP could decrease degradation production because a lower temperature would be required for aerosolization. Spectra were normalized using the PG and GL peaks so that degradation could be compared between samples. However, no significant and reproducible effect on degradation quantities was seen upon the addition of 5,10 , or $15 \mathrm{~mol} \%$ water (equivalent to $1.2,2.4$, and 3.6 vol\% added water) to equimolar PG+GL (Figure S1). We found that up to $15 \mathrm{~mol} \%$ added water had no significant impact on degradation production. The \%trapped aerosol ((absolute value of the change in the vial mass/absolute value of the change in tank mass)*100) was determined for each of the four conditions per tank and averaged. Samples from tank 1 contained $54 \pm 7 \%$ of the total aerosol produced. Samples from tanks 2 and 3 contained $37 \pm 4 \%$ and $44 \pm 2 \%$ of the total aerosol, respectively. 


\subsection{Conclusions}

The data obtained herein provide BP values of $\mathrm{PG}+\mathrm{GL}$ mixtures at 1 atmosphere pressure, and smooth fitting allows prediction of BP for any composition (e.g., Table 5). Depending on composition, the minimum temperature required to produce an e-cigarette aerosol from a PG+GL liquid ranges from $188.6{ }^{\circ} \mathrm{C}$ to $292^{\circ} \mathrm{C}$. Water, as an additive, was found to decrease the $\mathrm{BP}$ of $\mathrm{PG}+\mathrm{GL}$ mixtures for all tested mixtures. Nicotine $(3 \mathrm{~mol} \%$ ) was found to only significantly impact PG+GL mixtures containing at least $\sim 75 \mathrm{~mol} \%$ GL. The nicotine level tested ( $3 \mathrm{~mol} \%$ ) was equivalent to $\sim 64 \mathrm{mg} / \mathrm{mL}$; this exceeds common levels found in commercial e-liquids, which are often under $50 \mathrm{mg} / \mathrm{mL}$. In order to test the impact of BP lowering, a KangerTech KBOX Mini was tested at 22 watts using three different 1.2 X coils in three different KangerTech Subtank Minis at four different water conditions: $0,5,10$, and $15 \mathrm{~mol} \%$ added water. PG+GL degradation was monitored by ${ }^{1} \mathrm{H}$ NMR in terms of aldehydes and PG and/or GL formaldehyde hemiacetal production (Jensen et al., 2017, and Figure S1 herein). No significant differences in degradation were observed for the various added water conditions. Despite the $\sim 20^{\circ} \mathrm{BP}$ lowering of $\mathrm{PG}+\mathrm{GL}$ due to $5 \mathrm{~mol} \%$ water as determined using the microscale method, and likely an even greater BP decrease due to adding up to 15 mol\% water, these concentrations did not have a significant effect on solvent degradation. Larger amounts of water could reduce the BP of the solvent mixtures even more, and may then reduce solvent degradation; this set of studies is planned for other devices as well as varying the solvent mixtures beyond 50PG: $50 \mathrm{GL}$ (by mol\%). 


\subsection{Acknowledgments}

The authors thank Dr. Shankar Rananavare for suggestions regarding the Gibbs-

Konovalov parameterization; Michael Lethin and Astrid Woodall for assistance with the

boiling point measurements. The authors are also grateful to Alex Chally for expert

assistance with the syringe pump and vaping apparatus setup.

\subsection{Funding}

This work was supported by the U.S. National Institutes of Health under Grant

R01ES025257; research reported in this publication was supported by the NIEHS and FDA Center for Tobacco Products (CTP).

\subsection{ORCID}

Anna K. Duell http://orcid.org/0000-0001-5855-5162

James F. Pankow http://orcid.org/0000-0002-8602-9159

Samantha M. Gillette http://orcid.org/0000-00019899-9040

David H. Peyton http://orcid.org/0000-0001-5828-055X

\subsection{Contributions}

AKD conducted the work and contributed to the writing of the manuscript. 


\subsection{References}

Al-Jiboury, K. F. C. (2007). Correlation for fitting multicomponent vapor-liquid equilibria data and prediction of azeotropic behavior, Al-khwarizmi Eng. J., 3, 67 -86.

Chen, Y. C., Hung, S. K., Lee, H. Y., and Chien, I. L. (2015) Energy-saving designs for separation of a close-boiling 1,2-propanediol and ethylene glycol mixture, Ind. Eng. Chem. Res., 54, 3828-3843.

CORESTA; Routine Analytical Machine for E-Cigarette Aerosol Generation and Collection -Definitions and Standard Conditions. (2015). [Internet]. [accessed 2018 January 24]. Available from: https://www.coresta.org/ routine-analytical-machine-ecigarette-aerosol-generationand-collection-definitions-and-standard

Glycerine as a heat transfer fluid and antifreeze [Internet]. [accessed 2018 Jan 25]. Available from: http://www. hydratech.co.uk/uk/technical/Glycerine/60

Hahn, J., Monakhova, Y. B., Hengen, J., Kohl-Himmelseher, M., Sch€ussler, J., and Hahn, H., Thomas Kuballa, T., and Dirk W Lachenmeier, D. W. (2014). Electronic cigarettes: Overview of chemical composition and exposure estimation, Tob. Induc. Dis., $12,23$.

Jensen, R. P., Luo, W., Pankow, J. F., Strongin, R. M., and Peyton, D. H. (2015). Hidden formaldehyde in E-cigarette aerosols, N. Engl. J. Med., 372, 392-394.

Jensen, R. P., Strongin, R. M, and Peyton, D. H. (2017). Solvent chemistry in the electronic cigarette reaction vessel, Sci. Rep., 7, 42549.

Malesinski, W. (1965). Azeotropy and Other Theoretical Problems of Vapour-liquid Equilibrium, Interscience Publishers.

Market Research on Vapour Devices [Internet]. [accessed 2018 Jan 25]. Available from: http:// www.euromonitor.com/vapour-devices

Mickle, T. E-Cigarette Sales Rapidly Lose Steam. Wall Street Journal [Internet]. 2015 Nov 18 [accessed 2016 Jun 11]; Available from: http://www.wsj.com/articles/e-cigsalesrapidly-lose-steam-1447798921

Mnova j Mestrelab Research j [Internet]. [accessed 2018 Jan 26]. Available from: http://mestrelab.com/software/mnova/

Pendergrass, S. M. (1999). Determination of glycols in air: Development of sampling and analytical methodology and application to theatrical smokes, Am. Ind. Hyg. Assoc. J., 60, $452-457$.

Renewables 2016 Global status report [Internet]. [accessed 2016 Jun 10]. Available from: http://www.ren21.net/wpcontent/uploads/2016/06/GSR_2016_FullReport_pdf 
Richardson, A. (1886). LXXIII.-Determinations of vapour pressures of alcohols and organic acids, and the relations existing between the vapour-pressures of the alcohols and organic acids, J. Chem. Soc. Trans., 49, 761-776.

Schoenborn, C. A., and Gindi, R. M. (2015). Electronic cigarette use among adults: United States, 2014, National Center for Health Statistics data brief, 1-8.

Stull, D. R. (1947). Vapor pressure of pure substances. Organic and inorganic compounds, Ind. Eng. Chem., 39, 517-540.

Talih, S., Balhas, Z., Salman, R., El-Hage, R., Karaoghlanian, N., El-Hellani, A., Baassiri, M., Jaroudi, E., Eissenberg, T., Saliba, N., and Shihadeh, A. (2017). Transport phenomena governing nicotine emissions from electronic cigarettes: Model formulation and experimental investigation, Aerosol Sci. Technol., 51, 1-11.

Teschke, K., Chow, Y., Netten van, C., Varughese, S., Kennedy, S. M., and Brauer, M. (2005). Exposures to atmospheric effects in the entertainment industry, J. Occup.

Environ. Hyg., 2, 277-284.

Zhang, Y., Sumner, W., and Chen, D. R. (2013). In vitro particle size distributions in electronic and conventional cigarette aerosols suggest comparable deposition patterns, Nicotine Tob. Res., 15, 501-508. 


\subsection{Supporting Information}

Table S 1. Comparison Between Pre- and Post-Boiling Compositions in terms of Percent Glycerol (GL) for Propylene Glycol (PG) + GL Mixtures. Average Percent GL, N=3, as Determined by NMR (\%PG = 100\% - \%GL).

$$
\text { mol \% glycerol }
$$

$\begin{array}{ccc}\text { pre-boiling } & \text { post-boiling } & \text { |difference } \\ 0.0 & 0.0 & 0.0 \\ 8.3 & 8.3 & 0.0 \\ 16.8 & 17.3 & 0.5 \\ 25.8 & 26.2 & 0.4 \\ 35.2 & 35.8 & 0.6 \\ 44.7 & 45.6 & 0.9 \\ 55.0 & 55.9 & 0.9 \\ 65.4 & 66.4 & 1.0 \\ 76.3 & 77.6 & 1.3 \\ 87.7 & 89.2 & 1.5 \\ 99.9 & 99.9 & 0.0\end{array}$

PG+GL mixtures were prepared by mass; however, due to differences between pre- and post-boiling composition, the post-boiling molar quantities were used to relate boiling point and (mol\% and vol\%) compositions. The values for 0, 8.3, and $99.9 \mathrm{~mol} \% \mathrm{GL}$ $(0,10$, and $100 \mathrm{wt} \% \mathrm{GL})$ remained constant with respect to pre- and post-boiling composition, while the $16.8-87.7$ mol\% GL (20-90 wt\% GL) increased by $0.4-1.5$ mol\% with respect to mol\% GL. This effect is likely due to the lower vapor pressure of PG. The absolute difference between the pre- and post- boiling samples was calculated ("Idifferencel"). 
Table S 2. Boiling point variability for PG+GL mixtures between trials.

\begin{tabular}{ccccc} 
Average & \multicolumn{4}{c}{ Boiling point $\left({ }^{\circ} \mathbf{C}\right)$} \\
Mol\% Glycerol & Trial 1 & Trial 2 & Trial 3 & Average \pm SD \\
\hline $\mathbf{0} \pm \mathbf{0}$ & 188.0 & 188.9 & 189 & $188.6 \pm 0.6$ \\
$\mathbf{8 . 3} \pm \mathbf{0 . 1}$ & 191.5 & 191.9 & 191.5 & $191.6 \pm 0.2$ \\
$\mathbf{1 7 . 3} \pm \mathbf{0 . 2}$ & 194.4 & 195.1 & 194.5 & $194.7 \pm 0.4$ \\
$\mathbf{2 6 . 2} \pm \mathbf{0 . 2}$ & 198.4 & 198.8 & 198.5 & $198.6 \pm 0.2$ \\
$\mathbf{3 5 . 8} \pm \mathbf{0 . 2}$ & 203 & 203 & 203 & $203 \pm 0$ \\
$\mathbf{4 5 . 6} \pm \mathbf{0 . 1}$ & 207 & 208 & 208 & $208 \pm 0.6$ \\
$\mathbf{5 5 . 9} \pm \mathbf{0 . 4}$ & 214 & 214 & 214 & $214 \pm 0$ \\
$\mathbf{6 6 . 4} \pm \mathbf{0 . 4}$ & 223 & 223 & 222 & $223 \pm 0.6$ \\
$\mathbf{7 7 . 6} \pm \mathbf{0 . 4}$ & 236 & 236 & 236 & $236 \pm 0$ \\
$\mathbf{8 9 . 2} \pm \mathbf{0 . 2}$ & 258 & 259 & 258 & $258 \pm 0.6$ \\
$\mathbf{9 9 . 9} \pm \mathbf{0 . 1}$ & 292 & 292 & 292 & $292 \pm 0$
\end{tabular}


Table S 3. Difference between the boiling points proposed by the Antoine fit, and calculated values generated using the Gibbs-Konovalov parameterization

\begin{tabular}{|c|c|c|c|c|}
\hline$\underline{x_{\mathrm{GL}}}$ & $\begin{array}{l}\text { Antoine } \\
\underline{t\left({ }^{\circ} \mathrm{C}\right)}\end{array}$ & $\begin{array}{l}\text { Gibbs-Konovalov } \\
\text { calculated } \\
\underline{t\left({ }^{\circ} \mathrm{C}\right)}\end{array}$ & $\begin{array}{l}\text { difference } \\
\qquad \underline{t\left({ }^{\circ} \mathrm{C}\right)}\end{array}$ & \\
\hline 0 & 188 & 188.6 & 0.6 & \\
\hline 0.05 & 189.6 & 190.4 & 0.8 & \\
\hline 0.1 & 191.3 & 192.3 & 1.0 & \\
\hline 0.15 & 193.1 & 194.1 & 1.0 & \\
\hline 0.2 & 194.9 & 196.0 & 1.1 & \\
\hline 0.25 & 196.9 & 198.1 & 1.2 & \\
\hline 0.3 & 199.1 & $200(.2)$ & 1.1 & \\
\hline 0.35 & 201.4 & $202(.5)$ & 1.1 & \\
\hline 0.4 & 203.9 & 204(.9) & 1.0 & \\
\hline 0.45 & 206.7 & $207(.5)$ & 0.8 & \\
\hline 0.5 & 209.6 & $210(.3)$ & 0.7 & \\
\hline 0.55 & 212.9 & $213(.5)$ & 0.6 & \\
\hline 0.6 & 216.6 & $217(.1)$ & 0.5 & \\
\hline 0.65 & 220.7 & $221(.3)$ & 0.6 & \\
\hline 0.7 & 225.4 & $226(.3)$ & 0.9 & \\
\hline 0.75 & 230.9 & $232(.3)$ & 1.4 & \\
\hline 0.8 & 237.3 & $239(.8)$ & 2.5 & \\
\hline 0.85 & 245.1 & $248(.9)$ & 3.8 & \\
\hline 0.9 & 254.8 & $260(.3)$ & 5.5 & \\
\hline 0.95 & 267.8 & $274(.5)$ & 6.7 & \\
\hline 1 & 286.4 & $292(.0)$ & 5.6 & \\
\hline & & $\mathrm{mol} \%$ & $\begin{array}{l}1.8 \\
1.9\end{array}$ & $\begin{array}{l}\text { average } \underline{t\left({ }^{\circ} \mathrm{C}\right)} \text { difference } \\
\text { SD }\end{array}$ \\
\hline
\end{tabular}


The boiling point values obtained for PG+GL mixtures were fitted with a Gibbs-Konovalov parameterization. This parameterization was used to calculate the boiling point for PG+GL mixtures over the range of 0 to $100 \mathrm{~mol} \% \mathrm{GL}$, in increments of $5 \%$. These calculated boiling points ("Gibbs-Konovalov calculated $\left({ }^{\circ} \mathrm{C}\right)$ ") were compared with the Antoine equation calculated boiling points (“Antoine equation $\mathrm{t}\left({ }^{\circ} \mathrm{C}\right)$ )") to calculate the difference ("difference $\mathrm{t}\left({ }^{\circ} \mathrm{C}\right)$ "), where the difference = Gibbs-Konovalov calculated - Antoine. The average difference between the boiling points calculated using the Gibbs-Konovalov theorem and the Antoine equation were then averaged and resulted in an average absolute difference of $1.8 \pm 1.9^{\circ} \mathrm{C}(\mathrm{SD})$. 

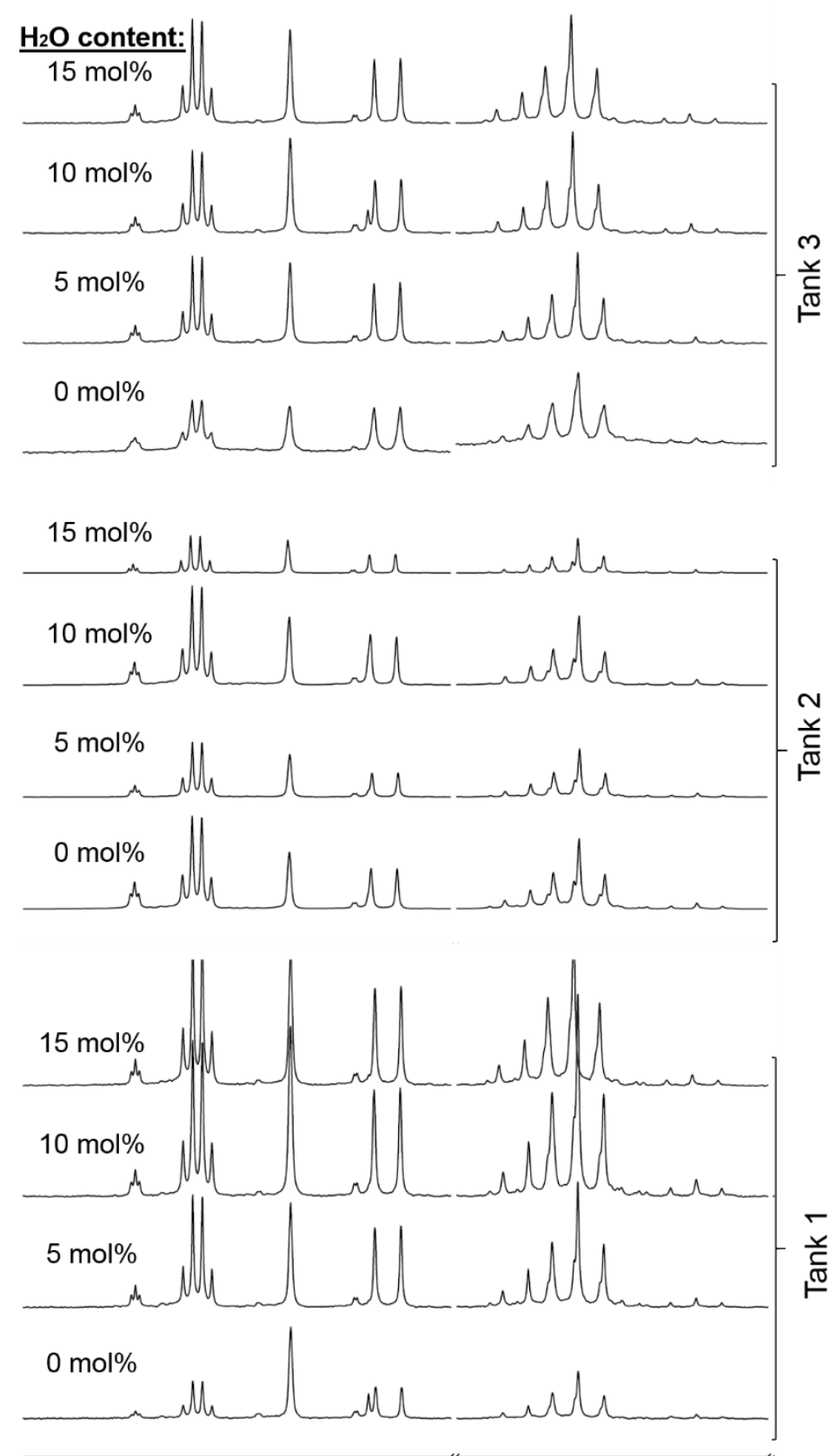

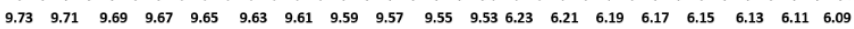

Figure S 1. ${ }^{1} \mathrm{H}$ NMR spectra showing degradation products produced by vaporizing PG:GL e-liquid containing varying amounts of water using three different coils. The following PG+GL degradation products can be identified using the chemical shifts of these products outlined by Jensen et al. (2017): propanal ( $\delta 9.68$, triplet), acetaldehyde $(\delta$ 9.65 , quartet), glyceraldehyde ( $\delta 9.62$, doublet), glycolaldehyde ( $\delta 9.61$, singlet), lactaldehyde ( $\delta 9.58$, doublet), acrolein ( $\delta 9.56$, doublet), and three different PG or GLbased formaldehyde hemiacetals ( $\delta 6.12$, triplet; $\delta 6.18,2$ overlapped triplets; $\delta 6.12$, triplet). 
Table S 4. The absolute value of the difference between the experimentally determined boiling points (average (AVE) \pm SD) and the Gibbs-Konovalov calculated values. The Gibbs-Konovalov parameters used can be found in Table 4. The average of the absolute values of the differences is $0.11^{\circ} \mathrm{C}$. The standard deviation of these values is $0.13{ }^{\circ} \mathrm{C}$.

\begin{tabular}{cccc}
\multicolumn{4}{c}{$B P\left({ }^{\circ} \mathrm{C}\right)$} \\
mol\% GL & Experimental AVE $\pm \mathrm{SD}$ & Gibbs-Konovalov Calculated & iDifferencel \\
\hline $0 \pm 0$ & $188.6 \pm 0.6$ & 188.6 & 0.03 \\
$8.3 \pm 0.1$ & $191.6 \pm 0.2$ & 191.6 & 0.00 \\
$17.3 \pm 0.2$ & $194.7 \pm 0.4$ & 195.0 & 0.31 \\
$26.2 \pm 0.2$ & $198.6 \pm 0.2$ & 198.6 & 0.02 \\
$35.8 \pm 0.2$ & $203 \pm 0.0$ & 202.8 & 0.17 \\
$45.6 \pm 0.1$ & $208 \pm 0.6$ & 207.8 & 0.13 \\
$55.9 \pm 0.4$ & $214 \pm 0.0$ & 214.1 & 0.09 \\
$66.4 \pm 0.4$ & $223 \pm 0.6$ & 222.6 & 0.07 \\
$77.6 \pm 0.4$ & $236 \pm 0.0$ & 236.0 & 0.00 \\
$89.2 \pm 0.2$ & $258 \pm 0.6$ & 258.3 & 0.00 \\
$99.9 \pm 0.1$ & $292 \pm 0.0$ & 291.6 & 0.39
\end{tabular}


3. Sucralose-Enhanced Degradation of Electronic Cigarette Liquids during Vaping

Anna K. Duell, ${ }^{\dagger}$ Kevin J. McWhirter, ${ }^{\dagger}$ Tetiana Korzun, ${ }^{\dagger}$ Robert M. Strongin, ${ }^{\dagger}$ and David H. Peyton* ${ }^{\dagger}$

†Department of Chemistry and Department of Civil and Environmental Engineering, Portland State University, Portland, Oregon 97207-0751, United States

Reprinted (adapted) with permission from:

Duell, Anna K., Kevin J. McWhirter, Tetiana Korzun, Robert M. Strongin, and David H. Peyton. "Sucralose-enhanced degradation of electronic cigarette liquids during vaping." Chemical research in toxicology (2019).

Copyright 2019 American Chemical Society. Further permissions related to the material excerpted should be directed to the ACS.

Link to source: https://pubs.acs.org/doi/abs/10.1021/acs.chemrestox.9b00047 


\subsection{Abstract}

Electronic cigarette liquids (e-liquids) with sweetener additives such as sucralose, a synthetic chlorinated disaccharide, are popular among some e-cigarette consumers; sucralose can be added either by the manufacturer or by the consumer. The prevalence of sucralose in commercial e-liquids is not known, nor is the typical concentration of sucralose when present; labels are not required to disclose ingredient information. Here, we report the effects of sucralose on e-liquid degradation upon e-cigarette vaping as studied using ${ }^{1} \mathrm{H}$ NMR spectroscopy, ion chromatography, and gas chromatography coupled with detection by mass spectrometry or flame ionization detector. Sucralose was found to be subject to degradation when included in propylene glycol + glycerol based eliquids and vaped; the presence of sucralose in the e-liquids also resulted in altered and enhanced solvent degradation. In particular, production of aldehydes (carbonyls) and hemiacetals (which have implications for health) was enhanced, as demonstrated by ${ }^{1} \mathrm{H}$ NMR. The presence of sucralose at $0.03 \mathrm{~mol} \%(0.14 \mathrm{wt} \%)$ in an e-liquid also resulted in production of potentially harmful organochlorine compounds and catalyzed the cyclization of aldehydes with solvents to acetals upon vaping; the presence of chloride in e-liquid aerosols was confirmed by ion chromatography. Quantities of sucralose as low as $0.05 \mathrm{~mol} \%(0.24 \mathrm{wt} \%)$ in e-liquids lead to significant production of solvent degradation products. 


\subsection{Introduction}

Sweeteners are common additives to conventional smoked and smokeless tobacco products, presumably to improve palatabilitiy. ${ }^{1,2}$ For electronic cigarettes (e-cigarettes), sweet and fruit-flavored e-cigarette liquids (e-liquids) are preferred over tobacco or menthol flavors by some former and current adult smokers, ${ }^{1}$ and fruit-flavored e-liquids in particular (which tend to be sweet) have been reported to be preferred by adolescents (less-so by adults). ${ }^{3}$ Chemicals added to enhance e-liquid sweetness include but are not limited to very low volatility compounds (e.g., steviol glycosides, mogrosides, sucrose, glucose, fructose, and sucralose), semivolatile compounds (e.g., maltol, ethyl maltol, and erythritol), and volatile flavor constituents (e.g., some esters, lactones, and aldehydes, which have often been described as "fruity"). ${ }^{4-8}$ Degradation of glucose and some other sugars have been shown to generate 5-hydroxymethylfurfural and furfural ${ }^{9-11}$ which are regarded as respiratory irritants ${ }^{12,13}$ and are volatile components in caramel/tobacco flavor profiles. ${ }^{14}$ For low volatility sweeteners such as glucose, volatile degradants may be more important for flavor profile enhancement than the parent compounds.

Sucralose, a synthetic sugar substitute commonly used in reduced-calorie foods, is a component of some e-liquids. Also, concentrated sucralose mixtures are also available from e-liquid companies so that consumers can add as much sucralose as desired to their e-liquids, which can be via a 5-15\% sucralose mixture in propylene glycol (PG). ${ }^{15}$ Similar in structure to sucrose, sucralose differs in that it contains three chlorines in place of three hydroxyls, and one $\mathrm{Cl}$ has an inverted stereochemistry relative to the hydroxyl it replaces in sucrose. These structural differences combine to make sucralose 400-700- 
fold sweeter by weight than sucrose. ${ }^{16}$ In e-cigarettes, sucralose has been reported to result in a small amount of sweetness enhancement, but this effect has been reported to be device-dependent,${ }^{17}$ perhaps because volatile flavor compounds may have a greater influence on perceived sweetness than sucralose. Although sucralose is regarded as safe for gastrointestinal consumption, ${ }^{18}$ neither sucralose itself nor its thermal degradation products have been shown to be safe upon inhalation.

Although there is little published regarding sucralose stability during vaping, this molecule has been examined for its inherent thermal stability and its stability in food products. Degradation of pure sucralose has been found to occur at temperatures as low as $\sim 98{ }^{\circ} \mathrm{C}$, as evidenced by the production of polychlorinated aromatic hydrocarbons; visible degradation was reported at $125{ }^{\circ} \mathrm{C} .{ }^{19}$ Degradation pathways for sucralose under stronger pyrolysis conditions at $250{ }^{\circ} \mathrm{C}$ and in the presence of glycerol (GL) (which is used in e-liquids) have been explored. ${ }^{20}$ Sucralose degradation (in glycerol) was proposed to occur via dehydration and dehydrochlorination reactions and was reported to generate hydrochloric acid, water, and chloropropanols. ${ }^{20}$ E-cigarettes are likely to achieve temperatures capable of degrading sucralose because the boiling points of the main solvents (PG and GL) and their mixtures range from $\sim 189$ to $292^{\circ} \mathrm{C} .{ }^{21}$

Here we used various techniques to study the chemical reactions occurring in sucralose-containing e-liquids upon vaping from a commercial tank-style e-cigarette. ${ }^{1} \mathrm{H}$ NMR excels at direct nondestructive analyses, especially for known compounds. ${ }^{22-25}$ In particular, the effect of sucralose on aldehyde (carbonyl) production, as indicated by levels of propanal, acetaldehyde, glycolaldehyde, and acrolein, as well as PG and/or GL 
formaldehyde hemiacetals can be monitored as formed when formaldehyde reacts with either PG or GL in a reversible reaction (i.e., formaldehyde can be released) and thus contribute to the level of total formaldehyde produced by an e-cigarette. Mass spectrometry (MS) methods were also used because low-concentration degradation products can be difficult to quantify by NMR, particularly when the resonances are overlapping or very near the resonances of high concentration-compounds, in this case the e-liquid solvents PG and GL. Gas chromatography/mass spectrometry (GC/ MS) allows for separation of known analyte compounds from the abundant PG and GL in the captured aerosol as well as identification of unknown compounds by way of the MS data. Thus, GC/MS allows quantification of cyclic acetals that could be undetected or underdetected by other techniques ${ }^{25}$ recent research indicates that cyclic acetals of common e-cigarette flavorants and PG exhibit different toxicological properties. ${ }^{26}$ Gas chromatography flame-ionization detection (GC/FID) is a complement to GC/MS by exhibiting a nearly proportional response with respect to the number of $\mathrm{C}$ atoms in each compound. ${ }^{27}$ Ion chromatography (IC) allows the direct determination of released chloride from sucralose during the vaping process.

\subsection{Materials and Methods}

\subsubsection{Chemicals and E-cigarette Devices}

For All Experiments. United States Pharmacopeia (USP) grade glycerol was obtained from Sigma-Aldrich (St. Louis, MO). Coils rated at $1.2 \Omega$ OCC (suggested by the manufacturer for use at 10-26 W) were used for all experiments (KangerTech US, LLC, Shenzhen, China). 
For ${ }^{1} H$ NMR Experiments. USP grade propylene glycol was purchased from Sigma-Aldrich. (S)-(-)-nicotine (99\%) was obtained from Alfa Aesar (Haverhill, MA). Sucralose (>98\%) and methanol (ACS grade) were obtained from TCI Co., Ltd. (Portland, OR). Details about methods used during sample collection for determination of free-base levels and degradation products have been reported previously. ${ }^{21,28}$

For GC/MS, GC/FID, and IC Experiments. USP grade propylene glycol was purchased from TCI. A commercially available "sweetener" (sucralose in PG) was purchased from EcigExpress (Bellingham, WA) in October of 2016. Aliquots of this commercial mixture were tested in triplicate via ${ }^{1} \mathrm{H}$ NMR and found to contain 3 mol \% (corresponding to $12 \mathrm{wt} \%$ or $8 \mathrm{vol} \%$ ) sucralose relative to PG as determined by integration analysis.

Sodium chloride (99.2\%) and HPLC-grade isopropanol (IPA) were purchased from Fisher Scientific (Pittsburgh, PA). 4-Methyl-1,3dioxolane (>98\%) (PG formaldehyde acetal), a mixture of 4hydroxymethy-1,3-dioxolane/5-hydroxy-1,3-dioxane (>98\%) (GL formaldehyde acetals), 3-chloropropan-1,2-diol (>98\%), and 1,3dichloropropan-2-ol (>98\%) were purchased from TCI. 1,2,3Trichlorobenzene (99\%) was purchased from Sigma-Aldrich. A KangerTech Subtank Nano was obtained from KangerTech. A Model NE-1010 (New Era Pump Systems Inc., Farmingdale, NY) syringe pump outfitted with a custom $300 \mathrm{~mL}$ syringe was used for sample puff generation. 


\subsubsection{E-liquid Preparation with the Compositions Confirmed by ${ }^{1} \mathrm{H}$ NMR}

Simplified e-liquids were prepared to contain 1:1 (by mol) PG and GL. Concentrations of sucralose ranged from 0.05 to $0.12 \mathrm{~mol} \%$ (equivalent to 0.24 to 0.57 wt \%) in the final liquid, as was found in commercial e-liquids tested by ${ }^{1} \mathrm{H}$ NMR. To evaluate coil and sample variability, the following e-liquids were prepared: sucralose-free PG+GL, $0.05 \mathrm{~mol} \%$ sucralose in PG+GL, and $0.10 \mathrm{~mol} \%$ sucralose in PG+GL. To test the effect of increasing amounts of sucralose on degradation using a single coil, the same 0.05 and $0.10 \mathrm{~mol} \%$ sucralose in PG+GL e-liquids were used but a $0.075 \mathrm{~mol} \%$ sucralose in PG+GL was also prepared. To assess the vaping impact of sucralose on the fraction of the nicotine in the free-base (unprotonated) state versus the monoprotonated state $\left(\alpha_{\mathrm{fb}}\right)$, a sample was prepared to contain $24 \mathrm{mg} / \mathrm{mL}$ nicotine (equivalent to $1.1 \mathrm{~mol} \%$ or $2.1 \mathrm{wt} \%$ ) in $\mathrm{PG}+\mathrm{GL}$; aliquots of this mixture were then combined with sucralose to obtain 0.12 mol \% sucralose. E-liquid compositions were verified by ${ }^{1} \mathrm{H}$ NMR peak integration.

\subsubsection{Sample Collection Protocol and ${ }^{1} \mathrm{H}$ NMR}

Vaporized e-liquid samples were collected following a protocol outlined previously,${ }^{21}$ using the modified CORESTA puffing method (where the power button was activated one second prior to each $3 \mathrm{~s}, 55 \mathrm{~mL}$ puff, with $27 \mathrm{~s}$ between puffs $).{ }^{29}$ This puff protocol was selected to be consistent with other researchers. The sample collection protocol for the determination of free-base nicotine content and subsequent ${ }^{1} \mathrm{H}$ NMR methods has been described. ${ }^{28}$ 
All coils $(1.2 \Omega)$ were conditioned with 10 puffs at $26 \mathrm{~W}$ prior to first-time sample collection, similar to prior studies. ${ }^{30-32}$ All other puffs (for wicking and sample collection) were generated at $20 \mathrm{~W}$. Either 10 or 20 "wicking puffs" were generated and discarded prior to aerosol sample collection with each new e-liquid.

Each degradation sample contained three puffs. Three different $1.2 \Omega$ coils were tested with three different sucralose concentrations $(0,0.05$, and $0.10 \mathrm{~mol} \%)$ to determine coil variability. Between e-liquid conditions, each tank was emptied of fluid and wiped down with lint-free tissues to minimize the residual e-liquid from the previous condition. Obvious excess e-liquid on the coil was removed, but the wicking material was not cleaned with solvent. The tank was then filled with the new e-liquid and 20 "wicking puffs" were generated to ensure that the previous e-liquid had been removed from the wicking material and had been replaced by the new e-liquid. For the coil and sample variability experiment, 3 samples were collected for each of 3 coils and each of the 3 eliquid compositions, for a total of 27 samples. A second experiment was conducted using a single coil and 4 different sucralose concentrations (0, 0.05, 0.075, and $0.10 \mathrm{~mol} \%$ sucralose in $\mathrm{PG}+\mathrm{GL}$ ) with 3 samples per condition to examine degradation trends for a total of 12 samples.

Each sample for determination of fraction free-base nicotine was derived from 15 puffs, following methods previously described. ${ }^{28}$ The same $1.2 \Omega$ coil was used to test the $24 \mathrm{mg} / \mathrm{mL}$ nicotine samples to eliminate variability between coils. Because of the cleaning of the coil between conditions, only 10 wicking puffs were generated prior to sample collection between e-liquids (without and then with $0.12 \mathrm{~mol} \%$ sucralose). 
Degradation samples were tested by ${ }^{1} \mathrm{H}$ NMR at $25^{\circ} \mathrm{C}$ and freebase nicotine samples were tested at $40{ }^{\circ} \mathrm{C}$ per previous methods. ${ }^{21,28}$ Spectra for degradation samples generated using sucralose-containing e-liquids frequently exhibited acid-induced broadening, likely because of $\mathrm{HCl}$ (a strong acid) production by degradation of sucralose, which made the hemiacetal degradation peaks minimally visible. To neutralize the acid, small quantities of DMSO- $d_{6}$ saturated with sodium bicarbonate were added to each sample until the hemiacetal peaks could be resolved. Spectra were normalized relative to the $\mathrm{PG}$ resonance at $\sim 1.05 \mathrm{ppm}$.

\subsubsection{Preparation of E-liquids, E-cigarette, and Calibration Standards for GC/MS, GC/FID, and IC Experiments}

An e-liquid containing $0.03 \mathrm{~mol} \%(0.14 \mathrm{wt} \%)$ sucralose was prepared by combining a 1:1 (by mol) PG/GL mixture with commercial sucralose "sweetener" (sucralose in PG). A KangerTech Subtank Nano was used with a KangerTech $1.2 \Omega$ OCC atomizer. The atomizer was "primed" per the manufacturer's instructions by saturating the inner wicking material with e-liquid. The tank was then filled to $80 \%$ capacity and left to wick for $\sim 30 \mathrm{~min}$.

A mixture of standards was prepared in IPA containing 4-methyl1,3-dioxolane, a mixture of 4-hydroxymethyl-1,3-dioxolane/5-hydroxy-1,3-dioxane, 3-chloropropan-1,2diol, and 1,3-dichloropropan2-ol. This mixture was used to prepare calibration standards at approximate concentrations of $200,100,50,10$, and $2 \mathrm{ng} / \mu \mathrm{L}$. Samples and calibration standards were spiked with 1,2,3-trichlorobenzene as a GC internal standard. 
A chloride stock solution was prepared using deionized water (MilliporeSigma; Burlington, MA) and sodium chloride (Fisher Scientific). IC calibration standards were made from the sodium chloride stock in $98 \% \mathrm{IPA} / 2 \% \mathrm{H}_{2} \mathrm{O}$ at approximate concentrations of $60,12,6,3$, and $1 \mathrm{ppm}(\mathrm{mg} / \mathrm{L})$ chloride.

\subsubsection{Sampling Methods for GC/MS, GC/FID, and IC}

The prepared Subtank Nano was installed on an Efusion DNA200 power supply (Lost Vape Ltd.; London, England), the resistance was confirmed to be $1.2 \pm 0.1 \mathrm{ohms,}$ and the power level was set to $20 \mathrm{~W}$. Puffs were generated using the $300 \mathrm{~mL}$ syringe pump. Each $5 \mathrm{~s}$ puff was $50 \mathrm{~mL}$ in volume and had an interpuff interval of $35 \mathrm{~s}$.

Aerosol generated by the e-cigarette was drawn through an $\sim 4.5 \mathrm{~cm}$ section of silicone tubing connected to an 18-gauge inlet needle which was inserted into a capped 2 $\mathrm{mL}$ autosampler vial. The orifice of the needle was positioned to impact aerosol particles against the vial wall. Another 18-gauge exit needle was positioned above the inlet needle and attached to an $\sim 8 \mathrm{~cm}$ length of tubing, connected to a solenoid valve, and then the syringe pump. An $\sim 7 \mathrm{~cm}$ length section of tubing connected the valve to the custom syringe.

Each aerosol sample consisted of three puffs. A total of 30 consecutive samples were collected for a total of 90 puffs. Samples were collected, then dispersed into $980 \mu \mathrm{L}$ of IPA and $20 \mu \mathrm{L}$ of GC internal standard solution (1,2,3-trichlorobenzene in IPA) $\sim 1 \mathrm{~h}$ after collection, giving a total volume of $\sim 1030-1050 \mu \mathrm{L}$, depending on the quantity of captured aerosol for each sample. A Teflon-lined screw cap was installed and each sample was mixed. An unvaped blank eliquid sample was prepared by diluting $50 \mu \mathrm{L}$ of 
the e-liquid in $930 \mu \mathrm{L}$ IPA with $20 \mu \mathrm{L}$ of internal standard solution. The unvaped blank was tested with experimental samples by GC/MS, GC/FID, and IC.

\subsubsection{Analytical Methods for GC/MS, GC/FID, and IC}

After sample collection, dilution, and the addition of an internal standard, sample vials along with calibration standards and blanks were tested by GC/MS and GC/FID, using the same sample order for both. After analysis by GC/FID, the contents of each vial were transferred to a $1.5 \mathrm{~mL}$ polypropylene IC vial and analyzed by IC. Additional solvent blanks for IPA and $\mathrm{H}_{2} \mathrm{O}$ were also tested using IC.

The limit of quantitation (LOQ) for free chloride IC samples was calculated using the standard deviation of free chloride detected in an unvaped e-liquid blank (0.004 ppm) multiplied by a factor of 10 resulting in a limit of $0.04 \mathrm{ppm}$. For compounds with mass concentrations estimated using total ion chromatogram (TIC) peak areas, which were normalized relative to internal standard TIC peak areas, the LOQ was estimated by the internal standard response factor and a minimum TIC peak area of 1000, resulting in the LOQ of $0.016 \mathrm{ng} / \mu \mathrm{L}$. The LOQ for compounds quantitated using GC/FID (multipoint calibration standard) was conservatively estimated to be one-tenth of the lowest concentration standard resulting in the following LOQs: 4-methyl-1,3-dioxolane (0.16 ng/ $\mu \mathrm{L})$, 4-hydroxymethyl-1,3-dioxolane ( $0.04 \mathrm{ng} / \mu \mathrm{L})$, 5-hydroxy-1,3-dioxane ( $0.2 \mathrm{ng} /$ $\mu \mathrm{L})$, and 3-chloro-1,2-propandiol $(0.24 \mathrm{ng} / \mu \mathrm{L})$.

\subsubsection{GC/MS}

Sample analyses were conducted using an Agilent 7890A GC equipped with a Restek 5Sil-MS column $(30 \mathrm{~m} \times 0.25 \mathrm{~mm} \mathrm{ID} \times 0.25 \mu \mathrm{m} \mathrm{df})$, which was coupled to an 
Agilent 5975C MSD. The autosampler injected $1 \mu \mathrm{L}$ of sample at a 10:1 split under 12 mL min-1 constant injector He flow (99.9999\% pure, AirGas; Radnor, PA). The injection port temperature was $200{ }^{\circ} \mathrm{C}$; after injection, the oven temperature was held at $40{ }^{\circ} \mathrm{C}$ for $2 \mathrm{~min}$, then increased at $10{ }^{\circ} \mathrm{C}$ per minute until it reached $300{ }^{\circ} \mathrm{C}$. The MS was operated in electron impact ionization mode using an ionization energy of $70 \mathrm{eV}$; detection was configured for positive ions scanning a range of 34-400 amu. The electron multiplier voltage was set to $1730 \mathrm{~V}$. Other conditions were interface temperature, 230 ${ }^{\circ} \mathrm{C}$; source temperature, $226{ }^{\circ} \mathrm{C}$; and quadrupole temperature, $150{ }^{\circ} \mathrm{C}$.

\subsubsection{GC/FID}

An Agilent 7890B GC with a Restek 5Sil-MS column $(30 \mathrm{~m} \times 0.25 \mathrm{~mm}$ ID $\times 0.25$ $\mu \mathrm{m}$ df) was used for flame ionization detection (FID). The same GC oven temperature program was used as described earlier in Section 3.3.7. GC/MS. Other conditions were injection volume, $1 \mu \mathrm{L}$; split ratio, 10:1 (He) at $16 \mathrm{~mL}$ min-1; injection port temperature, $200{ }^{\circ} \mathrm{C}$; detector temperature, $280{ }^{\circ} \mathrm{C}$; FID hydrogen flow, $30 \mathrm{~mL}$ min-1; FID air flow, $300 \mathrm{~mL}$ min-1; FID makeup gas (N2) flow, $25 \mathrm{~mL}$ min-1.

\subsubsection{Ion Chromatography}

All IC equipment, columns, and software used in this study were obtained from Dionex (Sunnyvale, CA). Anion analyses of samples were conducted using an ICS-5000 IC system outfitted with a conductivity detector cell and electrolytically regenerated suppressor (AERS 500, $4 \mathrm{~mm})$. Aliquots of samples $(25 \mu \mathrm{L})$ were injected into the system for each test. Separation was carried out using an IonPac-AS15 column with an 
IonPac-AG15 guard column and a flow of $1.20 \mathrm{~mL}$ min-1. An eluent concentration of 38 $\mathrm{mM}$ of potassium hydroxide was maintained for the entire $20 \mathrm{~min}$ run.

\subsection{Results and Discussion}
3.4.1. $\quad{ }^{1}$ H NMR of E-liquid Aerosol: Sample Variability, Device Differences, and Degradation Products

The extent of PG and GL degradation, as shown by the most abundant nonsolvent species detected (aldehydes) relative to the PG methyl resonance, was found to be consistent when using the same coil and e-liquid, both at zero and high tested sucralose concentrations (Figure 1). Different coils were shown to produce different concentrations of degradation products relative to PG (Figure 2). Replicates using a single coil produced highly consistent results, indicating that individual samples were representative of each eliquid condition. For the three coils, the average percent of the aerosol trapped in the sample vial for each condition ranged from 35 to $54 \%, 45-57 \%$, and $15-36 \%$ for the 0 , 0.05 , and $0.10 \mathrm{~mol} \%$ sucralose samples, respectively; similar to our previous results. ${ }^{23}$ As the sucralose concentration increased, the percent of the aerosol trapped decreased. The increase in degradation production observed with increased sucralose concentration reported herein is relative to the molar quantity of PG, rather than an absolute quantity of each degradation product. Because of this, it is possible that degradation production is underestimated by this method. 


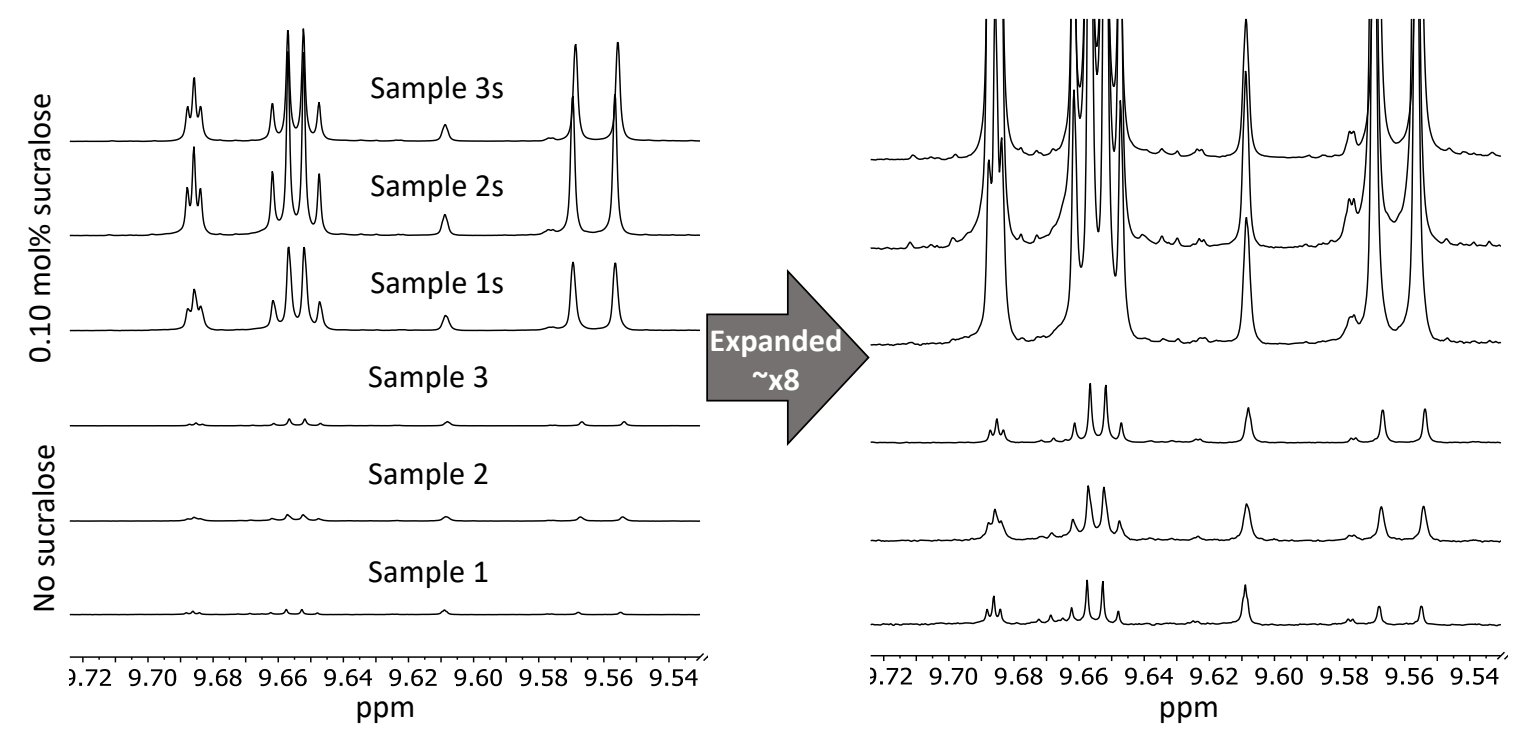

Figure 1. Sample degradant variability using the same coil with and without sucralose (0.10 mol\%) by ${ }^{1}$ H NMR. (L) Variability between samples collected using $0.10 \mathrm{~mol} \%$ sucralose e-liquid was found to be low. (R) The spectra were expanded to allow comparison to show the variability between samples collected without sucralose, and again found to be minimal. Samples were vaporized using the CORESTA puff method at 20 watts using a conditioned $1.2 \Omega$ coil. The intensities were relative to the PG methyl resonance. 


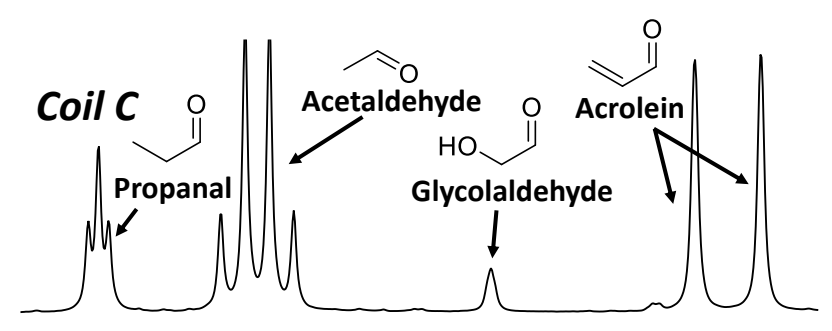

\section{Coil B}

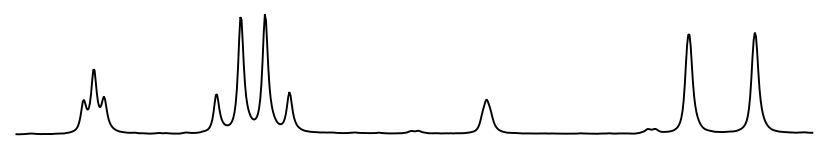

\section{Coil A}

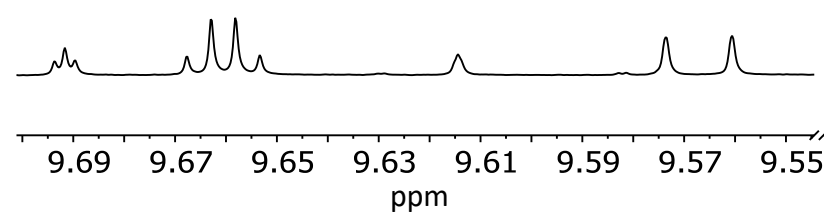

Figure 2. Degradant variability for 3 coils (A, B, \& C), all at $0.10 \mathrm{~mol} \%$ sucralose by ${ }^{1}$ H NMR. All spectra are normalized relative to the propylene glycol doublet at $\sim 1.05$ ppm. Samples were vaporized using the CORESTA puff method at 20 watts using a conditioned $1.2 \Omega$ coil. The intensities were relative to the PG methyl resonance. 
The effect of sucralose concentration $(0,0.05,0.075$, and $0.10 \mathrm{~mol} \%$ sucralose in PG+GL) on degradation produced by a single coil was compared by ${ }^{1} \mathrm{H}$ NMR. The concentrations of aldehydes (propanal, acetaldehyde, glycolaldehyde, and acrolein, Figure 3) and hemiacetals (Figure 4) all increased as sucralose concentration increased. Hemiacetals of formaldehyde with PG or GL are of concern because the formation reactions (Figure 5) are reversible: formaldehyde can be released by these hemiacetals and contribute to the total formaldehyde level delivered by an e-cigarette.23 Sucralose levels as low as $0.05 \mathrm{~mol} \%$ in PG+GL increased both aldehyde and hemiacetal output. Acid-induced broadening of $\mathrm{OH}$ resonances, including from the hemiacetals, was observed (likely due to the degradation of sucralose, which is known to produce hydrochloric acid ${ }^{19,20}$ as well as increase the production of other acids such as acetic acid), so sodium bicarbonate was added to sucralose-containing NMR samples to reduce the broadening until the hemiacetal peaks were visible (Figure 4). The average ( \pm SD) percent of the aerosol trapped in the sample vial for each condition $(0,0.05,0.075$, and 0.10 mol \% sucralose) was $48 \pm 4,36 \pm 4,16 \pm 5,18 \pm 4 \%$, respectively, all collected using the same coil. Again, increased sucralose concentration was found to result in a lower percent of the aerosol captured.

The protonation state of nicotine was evaluated before and after vaping, in order to assess the possible production of acid due to sucralose degradation. ${ }^{20}$ Nicotine can exist in nonprotonated (freebase) and protonated forms. Neglecting insignificant quantities of the diprotonated state, the fraction of nicotine in the free-base relative to the 
monoprotonated state $\left(\alpha_{\mathrm{fb}}\right)$ can be determined by ${ }^{1} \mathrm{H}$ NMR. ${ }^{28}$ The $\alpha_{\mathrm{fb}}$ value for the unvaped e-liquids used in this study (Figure 6) was $1.00 \pm \leq 0.01$.

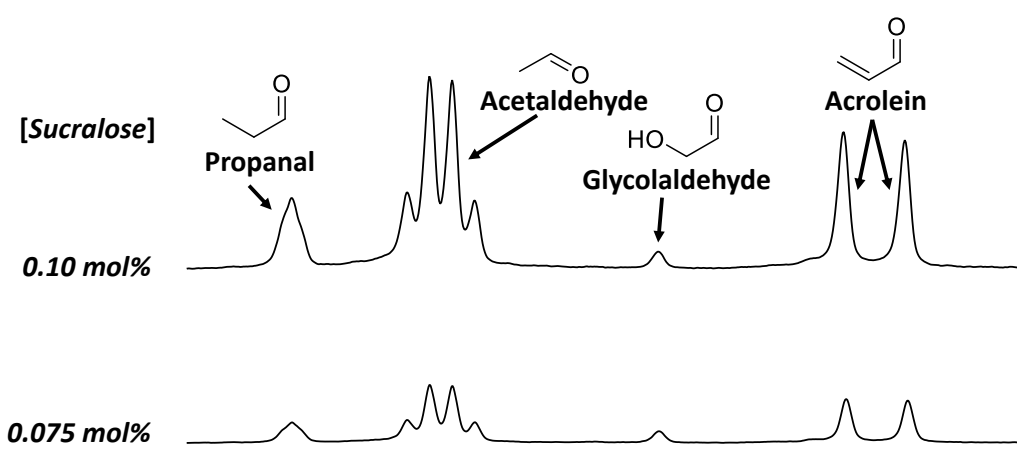

$0.05 \mathrm{~mol} \%$

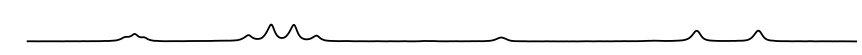

0 mol\%

\begin{tabular}{|c|c|c|c|}
\hline 9.70 & 9.68 & 9.66 & \\
\hline
\end{tabular}

Figure 3. Increased sucralose concentrations generated a greater concentration of aldehydes using the same device, coil, and vaping conditions by ${ }^{\mathbf{1}} \mathbf{H}$ NMR. Samples (3 puffs each) were generated at 20 watts using a conditioned $1.2 \Omega$ coil. The intensities were relative to the PG methyl resonance. 

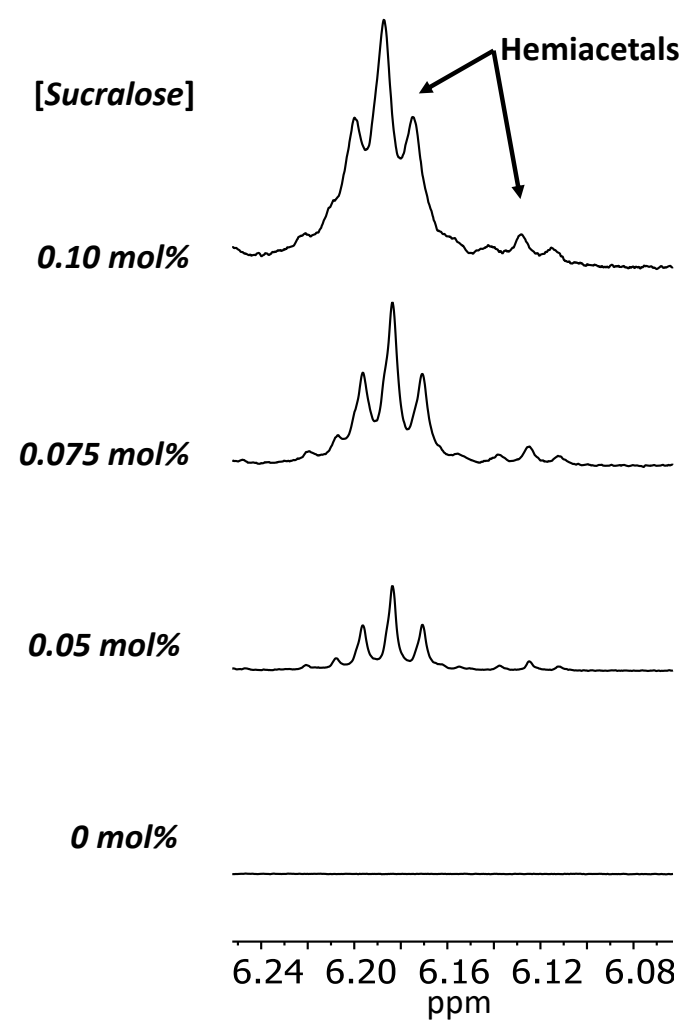

Figure 4. Increased sucralose concentrations generated a greater concentration of hemiacetals using the same device and coil by ${ }^{1} \mathrm{H}$ NMR. For these spectra, sodium bicarbonate (not present in the samples depicted in Figure 3) was added to buffer the mixture in order to slow the hydrogen exchange of the hemiacetal $\mathrm{OH}$ groups. Samples were vaporized using the CORESTA puff method at 20 watts using a conditioned $1.2 \Omega$ coil. The intensities were relative to the PG methyl resonance. 
<smiles>CC(O)CO</smiles>

propylene glycol

or glycerol<smiles>OCC(O)CO</smiles><smiles>CC(C)OCO</smiles>

isopropyl hemiformal (Isopropoxymethanol)<smiles>OC1COCOC1</smiles>

5-hydroxy-1,3-dioxane
O<smiles>CC(O)COCO</smiles><smiles>CC(CO)OCO</smiles>
+ formaldehyde

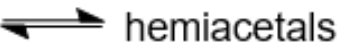<smiles>OCOCC(O)CO</smiles><smiles>OCOC(CO)CO</smiles><smiles>CC1COCO1</smiles>

4-methyl-1,3-dioxolane<smiles>CC1OCC(CO)O1</smiles>

4-hydroxymethyl-2methyl-1,3-dioxolane<smiles>OC1C2OC3OC1OC3O2</smiles><smiles>OCC1COCO1</smiles>

4-hydroxymethyl1,3-dioxolane<smiles>CC(C)OC(C)OC(C)C</smiles>

acetaldehyde diisopropyl acetal<smiles>OC(CCl)CCl</smiles>
1,3-dichloro-2-propanol

3-chloro-1,2-propandiol

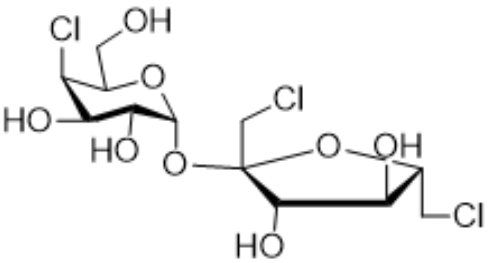

$\beta$-levoglucosan

1,6-dichloro-1,61,6-dichloro-1,6dideoxyfructoside dideoxyfructose<smiles>OC1[C@H](O)[C@@H](CCl)OC1(O)CCl</smiles><smiles>OCC(O)CCl</smiles><smiles>O=C(CCl)[C@H](O)[C@H](O)[C@H](O)CCl</smiles>

Figure 5. Propylene glycol- and glycerol-based hemiacetal production, ${ }^{23}$ other degradation products identified in this study, and related structures. 


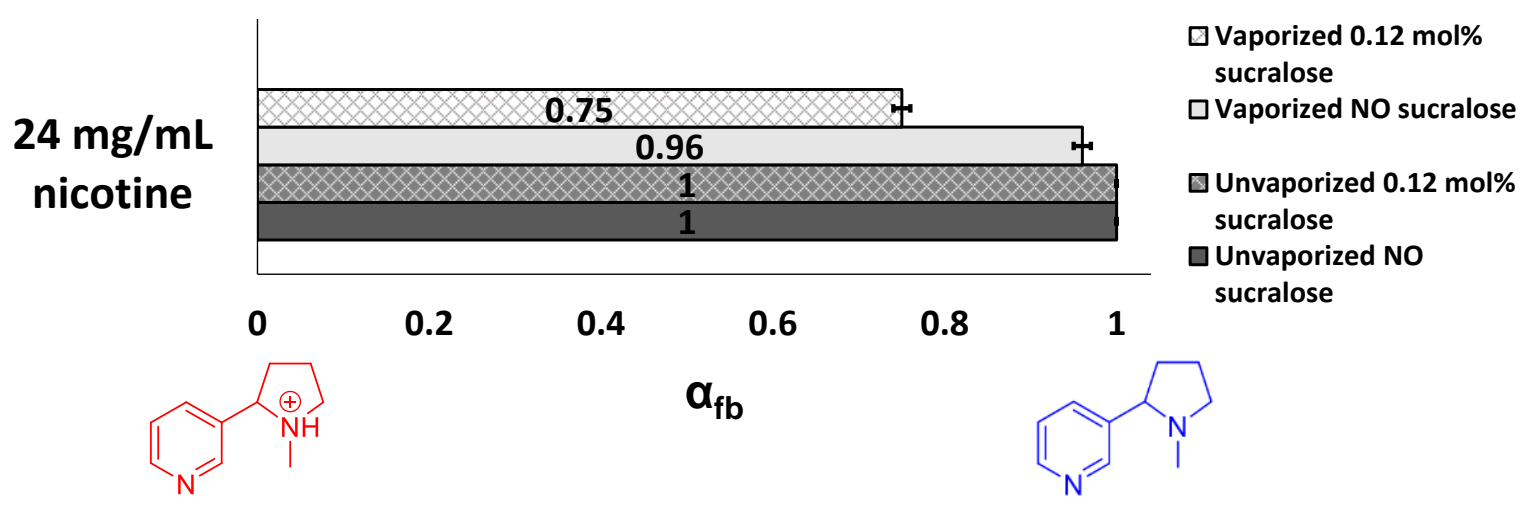

Figure 6. Fraction of nicotine in the free-base state $\left(\alpha_{\mathrm{fb}}\right)$ in e-liquids (PG, GL, and nicotine) before and after being vaporized with and without sucralose $(0.12 \mathrm{~mol} \%)$ as determined using differences in ${ }^{1} \mathbf{H}$ NMR chemical shifts. The same $1.2 \Omega$ coil was used to vaporize all e-liquids shown. Samples were vaporized using the CORESTA puff method at 20 watts using a conditioned coil, and collected directly into a micro-NMR tube.

Vaping the $24 \mathrm{mg} / \mathrm{mL}$ nicotine-containing PG+GL mixture (no sucralose) produced aerosol characterized by $\alpha_{\mathrm{fb}}=0.96 \pm 0.01$. Vaping the same e-liquid, but with $0.12 \mathrm{~mol} \%$ sucralose added produced aerosol characterized by $\alpha_{\mathrm{fb}}=0.75 \pm 0.01$. The change in degree of protonation for nicotine was then used to calculate the approximate number of protons produced due to the presence of sucralose during vaporization, possibly as $\mathrm{HCl}^{20}$ The $\alpha_{\mathrm{fb}}$ for the vaporized $24 \mathrm{mg} / \mathrm{mL}$ nicotine samples decreased by 0.21 (0.96 to 0.75$)$ when the sucralose was added; if this decrease in $\alpha_{\mathrm{fb}}$ is entirely attributed to sucralose, an average of $\sim 2.2$ protons would be released from every vaped sucralose molecule. This is consistent with the pyrolysis mechanism proposed by Rahn and Yaylayan, which suggested that each sucralose molecule should release 2 equiv of hydrochloric acid. ${ }^{20}$ The extra 0.2 protons taken up by nicotine may be from other acids (such as acetic acid) that may be produced during degradation. Possible evidence for this includes the enhanced solvent degradation after the addition of sucralose (Figures 1, 3, and 4), that more acetate 
was observed in the high sucralose concentration samples (not shown), and that the samples depicted in Figure 4 required addition of a sodium bicarbonate in order to slow the acid-catalyzed exchange of $\mathrm{OH}$ groups for NMR detection. The $\alpha_{\mathrm{fb}}$ results show that more acid is being produced with the addition of $0.12 \mathrm{~mol} \%$ sucralose than without. Sucralose thus appears to increase acid production from PG and GL degradation, including hydrochloric acid, directly during the breakdown. One outcome of sucralose degradation producing acid(s) could be to reduce $\alpha_{\mathrm{fb}}$ for nicotine, making the e-liquid less harsh. ${ }^{28}$ This warrants further study.

\subsubsection{Aerosol Collection and Target Analytes for GC/ MS, GC/FID, and IC Analyses}

The average $( \pm \mathrm{SD})$ mass of aerosol condensate collected in each vial (three puffs per vial), considering all samples, was $37 \pm 12 \mathrm{mg}$. The range was $21-52 \mathrm{mg}$, indicating that there was variability in the mass captured. Overall, for all the puffs $1.56 \mathrm{~g}$ of the starting e-liquid material was vaporized and $1.12 \mathrm{~g}$ of aerosol condensate was collected (30 vials, 90 puffs total) resulting in an overall capture efficiency of $72 \%$. Total capture efficiency was calculated using tank mass after 90 puffs and tank starting mass versus total mass collected in all vials.

Results for target analytes (structures in Figure 5) are given in Table 1. Observed products included direct sucralose degradation products (1,6-dideoxy-1,6dichlorofructose and free chloride) as previously reported,20,33 formaldehyde (4methyl1,3-dioxolane; 4-hydroxymethyl-1,3-dioxolane; 5-hydroxy-1,3-dioxane), and acetaldehyde acetals (4-hydroxymethyl-2-methyl-1,3-dioxolane) (structures in Figure 5) which are promoted to form under acidic conditions, reaction products between 
hydrochloric acid and GL reaction products (3-chloropropan-1,2-diol) as well as a marker of cellulose degradation (levoglucosan). An acetal of acetaldehyde and isopropanol (acetaldehyde diisopropyl acetal) and the hemiacetal of formaldehyde and isopropanol (IPA hemiformal) were also observed.

Formaldehyde acetals and acetaldehyde acetals could be formed with e-liquid solvents PG and GL during aerosol generation and/or sample condensation. ${ }^{22,23}$ Aldehydes are known to react with alcohols to form acetals through an acid catalyzed mechanism..$^{26,34}$ The presence of acetals supports the assertion that acids are formed during the vaporization process.

As formaldehyde and acetaldehyde are highly volatile and expected to favor partitioning into the aerosol gas phase 35 it is more likely that these carbonyls were present in the particle phase of the collected aerosol as less volatile hemiacetals with PG or GL. ${ }^{22-25}$ Acetaldehyde diisopropyl acetal and IPA formaldehyde hemiacetal are therefore more likely to have formed after addition of IPA where exchange could occur with existing acetaldehyde/formaldehyde hemiacetals of PG and/or GL (see Supporting Information for additional information on the identification of IPA-formaldehyde hemiacetal).

The IC results showed the presence of free chloride in the vaporized samples, ostensibly as hydrochloric acid. ${ }^{19,20}$ Considering samples $25-30$, only a small amount $(0.005 \mu \mathrm{mol} \mathrm{g-1)}$ of free chloride was detected in the starting material before vaporization compared to a total of $0.397 \mu \mathrm{mol} \mathrm{g}-1$ detected in the aerosol condensate samples, an $\sim 80$-fold increase. The presence of free chloride in e-cigarette aerosol 
indicates that sucralose is unstable in the e-cigarette environment tested, which was within bounds of the settings recommended by the manufacturer for this coil, device, and PG to GL ratio. This is likely because the boiling points for PG +GL mixtures (must meet or exceed these for vaporization) range from 188.6 to $292^{\circ} \mathrm{C}$, with 50:50 (by mol) PG and GL boiling at $210{ }^{\circ} \mathrm{C},{ }^{21}$ which well exceeds the temperature at which pure sucralose has been shown to degrade, $125^{\circ} \mathrm{C} .{ }^{19}$ 
Table 1. Compounds detected ( $\mu$ g per mg aerosol condensate). ${ }^{a}$

\begin{tabular}{|c|c|c|c|}
\hline Compound Name ${ }^{b}$ & $\begin{array}{c}\text { Low } \\
\left(\mu \mathrm{g} \mathrm{mg}^{-1}\right)\end{array}$ & $\begin{array}{c}\text { High } \\
\left(\mu g \mathrm{mg}^{-1}\right)\end{array}$ & $\begin{array}{l}\text { Average } \pm \text { SD } \\
\left(\mu g \mathrm{mg}^{-1}\right)\end{array}$ \\
\hline IPA formaldehyde hemiacetal ${ }^{\mathrm{d}, \mathrm{h}}$ & 2820 & 3780 & $3360 \pm 340$ \\
\hline 4-methyl-1,3-dioxolane ${ }^{c}$ & 121 & 163 & $141 \pm 17$ \\
\hline 4-hydroxymethyl-1,3-dioxolane ${ }^{c}$ & 84 & 127 & $104 \pm 18$ \\
\hline 5-hydroxy-1,3-dioxane ${ }^{c}$ & 292 & 413 & $373 \pm 42$ \\
\hline 4-hydroxymethyl-2-methyl-1,3-dioxolane ${ }^{d}$ & 156 & 183 & $168 \pm 9$ \\
\hline acetaldehyde diisopropyl acetal $^{d}$ & 336 & 621 & $469 \pm 99$ \\
\hline free chloride ${ }^{f}$ & 44 & 68 & $57 \pm 10$ \\
\hline 3-chloro-1,2-propandiol c & 7.6 & 11.8 & $9.5 \pm 1.4$ \\
\hline 1,6-dichloro-1,6-dideoxyfructose ${ }^{d}$ & 3.5 & 5.4 & $4.3 \pm 0.8$ \\
\hline unidentified chlorinated compound $\mathrm{e}, \mathrm{g}$ & 1.4 & 2.0 & $1.7 \pm 0.2$ \\
\hline$\beta$-levoglucosan ${ }^{d}$ & 29 & 34 & $32 \pm 2$ \\
\hline
\end{tabular}

${ }^{a}$ Detection range of target compounds represented as $\mu \mathrm{g}$ per $\mathrm{mg}$ aerosol condensate collected. as determined by GC/MS, GC/FID, or IC (samples 25-30, generated using a $1.2 \Omega$ coil at 20 watts containing comprised of 1:1 molar propylene glycol and glycerol). Free chloride was the only target analyte detected in an unvaporized starting material blank (Table 2).

${ }^{\mathrm{b}}$ Structures are depicted in Figure 5.

${ }^{c}$ Multipoint calibration prepared from authentic chemical standards, GC/FID peak area normalized to internal standard peak area and mass concentration calculated.

${ }^{\mathrm{d}}$ Analyte mass concentration estimated from GC/MS Total Ion Chromatogram (TIC) peak area normalized to internal standard TIC peak area.

${ }^{\mathrm{e}}$ Multipoint calibration standard used to establish response factor from 1,3dichloropropan-2-ol, target ion peak area extracted from GC/MS data and normalized to internal standard extracted target ion peak area to estimate approximate analyte mass concentration.

${ }^{\mathrm{f}}$ IC used with multipoint calibration standard prepared from sodium chloride.

g Spectral match identified 1,3-dichloropropanol with high certainty but retention time did not match that of an authentic chemical standard. Spectra suggest this unidentified compound is at least monochlorinated.

${ }^{\mathrm{h}}$ See Supporting Information for details on identification. 
IC results (Table 1) for sample numbers 25-30 (at the end of the experiment) show that during vaporization, each sucralose produces an average of $\sim 0.9$ free chlorides. Differences between IC findings and NMR results are like due to IC measuring the chloride concentrations as well as the formation of organochlorine compounds detected by GC/MS (Table 1), whereas NMR was used to determine the presence of protons accepted by nicotine. Another difference involves the presence of nicotine, which was only used in the NMR experiments. Other differences include the concentration of sucralose used, as well as sample collection protocol. In general, both the IC and NMR results indicate that sucralose degrades, leading likely production of $\mathrm{HCl}$.

Samples of the unvaped e-liquid starting material contained no detectable levels of 1,6-dideoxy-1,6-dichlorofructose (a known sucralose hydrolysis product) providing evidence that its formation must have occurred during the vaporization process rather than in the heated zones of the GC or MS.

As discussed previously, chloropropanols have been demonstrated to form when sucralose is heated in the presence of glycerol under pyrolysis conditions, which is especially relevant to e-cigarettes where glycerol is a ubiquitous solvent. ${ }^{20}$ While the total amount of 3-chloropropan-1,2-diol detected in samples 25-30 ( 10 $\mu \mathrm{g} \mathrm{g}^{-1}$ of e-liquid vaporized) was below a threshold of concern (European Commission tolerable daily intake of $2 \mu \mathrm{g} / \mathrm{kg}$ body weight), ${ }^{36}$ it should be noted that there is no literature to date on the effects of 3-chloropropan-1,2-diol inhalation though it is considered a Group 2B possible human carcinogen. 
Vaporization of e-liquids containing sucralose may degrade the atomizer wicking material, which is comprised of cellulose (advertised as "organic cotton") for the atomizers used in this study. For samples $25-30, \sim 32 \mu \mathrm{mols} \mathrm{mg}^{-1} \beta$-levoglucosan were captured, far exceeding $\sim 3 \times 10-3 \mu \mathrm{mols} \mathrm{mg}^{-1}$ sucralose in the starting material. This suggests that sucralose was not a major source of $\beta$-levoglucosan captured in samples. One of the main thermal degradation products of cellulose $(a \beta(1 \rightarrow 4)$ linked polymer of D-glucose) is levoglucosan which is generated from hydrolyzed D-glucose units through dehydration. ${ }^{37}$ In addition, production of levoglucosan from cellulose can be catalyzed by acids. ${ }^{38}{ }^{1} \mathrm{H}$ NMR, GC, and IC results support the generation of acids. Therefore, it is conceivable that the majority of levoglucosan detected arose from acid catalyzed degradation of the cellulose wicking material in the atomizer. This is likely a key factor in the "coil killer" properties of sucralose. D-glucose (which is nonvolatile) is unlikely to be carried away by vaporization and instead left to further degrade into compounds such as hydroxymethylfurfural (HMF) and furfural, which are volatile. ${ }^{11}$ Production of HMF and furfural may not be an unpleasant experience for the consumer as they are components in caramel/tobacco flavor profiles. ${ }^{14}$

Some proponents of e-cigarettes have claimed that consumers may discontinue use of a particular coil/e-liquid if exposed to significant degradants and have dismissed degradation findings as having been produced under unrealistic conditions ${ }^{39}$ However, it is possible that consumers build a tolerance to irritating substances, may even seek a level of irritation, ${ }^{32,33}$ and in many cases continue consumption due to nicotine addiction despite harm. ${ }^{40,41}$ E-liquid components (nicotine, cinnamaldehyde, and menthol) and 
degradation products (formaldehyde and acrolein) are known agonists of TRPA1 ion channels, which respond to irritants. ${ }^{42-46}$ There have been reports that chronic exposure to irritants can desensitize the response, indicating that e-liquids containing nicotine, menthol, and/or cinnamaldehyde could potentially lower a consumer's sensitivity to toxic e-liquid degradants. ${ }^{45,47}$ Some level of PG and/or GL degradation, especially the formation of acetaldehyde, may even be desirable for some consumers. Acetaldehyde has been demonstrated to react with biogenic amines to form monoamine oxidase inhibiting compounds which act synergistically with nicotine..$^{48}$

\subsubsection{Forms of Chlorine Released from Sucralose}

Approximately $1 \%$ of the total possible chloride produced by sucralose was accounted for as organic compounds determined by GC methods (Table 2). Because sucralose is a very low volatility compound, it is likely that much of the sucralose in the e-liquid was simply not vaporized along with the PG and GL and perhaps concentrated in the wicking material. Unidentified organochlorine compounds could be a source of unaccounted for chlorine such as the unidentified chlorine compound noted in Table 1. When pure sucralose is heated, it has been demonstrated to generate organochlorine compounds volatile enough to be collected from the headspace gas phase. ${ }^{19}$ These compounds include a chlorinated furan derivative, a chlorinated tetrahydropyran, and a polychlorinated aromatic hydrocarbon. ${ }^{19}$ Of the compounds identified by de Oliveira et al., ${ }^{19}$ only the chlorinated furan (originating from the fructose moiety of sucralose) was identified by GC/MS in the present study (as 1,6-dichloro-1,6-dideoxyfructose, before dehydration to a furan derivative ${ }^{20}$ ) with certainty. Using infrared spectroscopy, de 
Oliveira et al. identified chloroacetaldehyde generated during the heating of pure sucralose. ${ }^{19}$ Chloroacetaldehyde, which readily forms acetals in the presence of alcohols, was not identified in the present study though it may be related to the unidentified chlorinated compound in Table 1. Some reactions/pathways for sucralose degradation in the electronic cigarette setting are undoubtedly different than those in the pyrolysis of pure sucralose due to the addition of PG and GL in an e-cigarette as well as the temperature/ environmental differences.

Table 2. Total chlorine found from samples containing sucralose. ${ }^{\mathrm{a}}$

\begin{tabular}{lccc} 
& \multicolumn{2}{c}{$\mu$ mol chlorine equivalents } & \multirow{2}{*}{ \% of total } \\
\cline { 2 - 3 } Compound & Unvaporized & Captured & \\
\hline sucralose & 1.39 & ND $^{c}$ & ND $^{c}$ \\
free chloride & 0.005 & 0.397 & 28.4 \\
3-chloropropan-1,2-diol & ND $^{c}$ & 0.012 & 0.9 \\
1,6-dichloro-1,6-dideoxyfructose & ND $^{c}$ & 0.005 & 0.4
\end{tabular}

${ }^{a} \mu$ mol chlorine equivalents ${ }^{b}$ of chlorinated target compounds detected in captured aerosol condensate compared to the amount in unvaporized starting material as determined by GC/MS, GC/FID, or IC (samples 25-30, generated using a $1.2 \Omega$ coil at 20 watts containing comprised of 1:1 molar propylene glycol and glycerol).

${ }^{\mathrm{b}}$ Each $\mu \mathrm{mol}$ of free chloride and 3-chloropropan-1,2-diol each contribute one chlorine equivalent, while 1,6-dichloro-1,6-dideoxyfructose contributes two chlorines, while sucralose contributes three.

${ }^{\mathrm{c}}$ ND: Not detected. 


\subsection{Conclusions}

This study provides evidence that sucralose is unstable in the e-cigarette environment tested, as evaluated within the bounds of the settings recommended by the manufacturer of the contemporary device that was used. The vaporization of a sucralosecontaining e-liquid ( $0.05 \mathrm{~mol} \%$, equivalent to $0.24 \mathrm{wt} \%$, or greater) was found by NMR to increase the production of aldehydes (such as propanal, acetaldehyde, glycolaldehyde, and acrolein) as well as formaldehyde hemiacetals (which can release formaldehyde). Analysis by GC/MS and GC/FID showed that chloropropanols (3chloropropan-1,2-diol) were formed during vaporization for sucralose-containing e-liquids $(0.03 \mathrm{~mol} \%$, equivalent to $0.14 \mathrm{wt} \%$, sucralose). The use of IC confirmed that while a small portion of the total possible chlorines on sucralose was liberated during e-liquid vaporization, chloropropanols were still formed and free chloride was detected. The presence of free chloride indicates that sucralose is unstable in e-liquids when vaporized, ${ }^{19,20}$ and the presence of acid was confirmed using NMR by determining the protonation state of nicotine before and after vaporization. Production of acid from sucralose degradation likely enhances aldehyde and hemiacetal formation from PG and/or GL during vaporization due to the acid catalyzed nature of these degradation pathways. By NMR it was determined that $\sim 2.2$ protons were absorbed by nicotine after vaping a sucralosecontaining e-liquid $(0.12 \mathrm{~mol} \%$ sucralose and $24 \mathrm{mg} / \mathrm{mL}$ nicotine which is equivalent to $1.1 \mathrm{~mol} \%$ ). IC analysis of samples $25-30$ indicated that an average of $\sim 0.9$ free chlorides were released per sucralose molecule when vaping a sucralose-containing eliquid ( $0.03 \mathrm{~mol} \%$ sucralose). This apparent difference may be attributed not only to the 
different concentrations of sucralose (the NMR-based data were for a higher concentration of sucralose, which induces a greater overall level of total degradation) but also that the NMR experiments examined the uptake of protons by nicotine and IC allows detection of free chloride, rather than protons. Because of the increase in e-liquid degradation and the production of chloropropanols, the use of sucralose in e-liquids should be avoided; the presence of sucralose as an ingredient in commercial e-liquids should be disclosed by means of appropriate labeling.

\subsection{Author Information}

\subsubsection{Corresponding Author}

*E-mail: peytond@pdx.edu.

\subsubsection{ORCID}

Anna K. Duell: 0000-0001-5855-5162

Robert M. Strongin: 0000-0003-3777-8492

David H. Peyton: 0000-0001-5828-055X

\subsubsection{Author Contributions}

The manuscript was written through contributions of all authors. All authors have given approval to the final version of the manuscript.

\subsection{4. $\quad$ Funding}

This work was supported by the U.S. National Institutes of Health, Grant R01ES025257; the research reported was supported by the NIEHS and FDA Center for Tobacco 
Products (CTP). The content is solely the responsibility of the authors and does not necessarily represent the views of the NIH or the FDA.

\subsubsection{Notes}

The authors declare no competing financial interest.

\subsection{Acknowledgments}

The authors thank Mr. Ari J. Bluffstone for experimental assistance and Professor James F. Pankow for his critical reading of and suggestions for this manuscript.

\subsection{Abbreviations List}

$\alpha_{\mathrm{fb}}$, fractional free-base nicotine content; e-cigarette, electronic cigarette; e-liquid, electronic cigarette liquid; FID, flame ionization detector; GC/MS, gas chromatography/mass spectrometry; GC/FID, gas chromatography with flame ionization detector; IC, ion chromatography (IC); GC, gas chromatography; GL, glycerol; HMF, hydroxymethylfurfural; IPA, isopropanol; MS, mass spectrometry; NMR spectroscopy, nuclear magnetic resonance spectroscopy; PG, propylene glycol; IPA, isopropyl alcohol; HPLC-MS/MS, high performance liquid chromatography tandem mass spectrometry

\subsection{Contributions}

AKD conducted the NMR spectroscopy work, NMR analysis, and contributed to the writing of the manuscript. 


\subsection{References}

(1) Farsalinos, K. E., Romagna, G., Tsiapras, D., Kyrzopoulos, S., Spyrou, A., and Vouthis, V. (2013) Impact of Flavour Variability on Electronic Cigarette Use Experience: An Internet Survey. Int. J. Environ. Res. Public Health 10 (12), 7272-7282.

(2) Felton, R. E. (1972) What flavoring can do to improve sales of tobacco products. World Tobacco 38, 147-148.

(3) Morean, M. E., Butler, E. R., Bold, K. W., Kong, G., Camenga, D. R., Cavallo, D. A., Simon, P., O’Malley, S. S., and Krishnan-Sarin, S. (2018) Preferring more e-cigarette flavors is associated with ecigarette use frequency among adolescents but not adults. PLoS One 13 (1), No. e0189015.

(4) Behar, R. Z., Luo, W. T., McWhirter, K. J., Pankow, J. F., and Talbot, P. (2018) Analytical and toxicological evaluation of flavor chemicals in electronic cigarette refill fluids. Sci. Rep. 8, 8.

(5) Fagan, P., Pokhrel, P., Herzog, T. A., Moolchan, E. T., Cassel, K. D., Franke, A. A., Li, X., Pagano, I., Trinidad, D. R., Sakuma, K. K., Sterling, K., Jorgensen, D., Lynch, T., Kawamoto, C., Guy, M. C., Lagua, I., Hanes, S., Alexander, L. A., Clanton, M. S., Graham-Tutt, C., Eissenberg, T., and Addictive Carcinogens, W. (2018) Sugar and Aldehyde Content in Flavored Electronic Cigarette Liquids. Nicotine Tob. Res. 20 (8), 985-992.

(6) Kubica, P., Wasik, A., Kot-Wasik, A., and Namiesnik, J. (2014) An evaluation of sucrose as a possible contaminant in e-liquids for electronic cigarettes by hydrophilic interaction liquid chromatography-tandem mass spectrometry. Anal. Bioanal. Chem. 406 (13), 3013-8.

(7) Miao, S., Beach, E. S., Sommer, T. J., Zimmerman, J. B., and Jordt, S. E. (2016) High-Intensity Sweeteners in Alternative Tobacco Products. Nicotine Tob. Res. 18 (11), 2169-2173.

(8) Tierney, P. A., Karpinski, C. D., Brown, J. E., Luo, W., and Pankow, J. F. (2016)

Flavour chemicals in electronic cigarette fluids. Tob Control 25 (e1), No. e10.

(9) Capuano, E., and Fogliano, V. (2011) Acrylamide and 5-hydroxymethylfurfural (HMF): A review on metabolism, toxicity, occurrence in food and mitigation strategies. Lwt-Food Sci. Technol. 44 (4), 793-810.

(10) Jing, Q., and Lu, X. Y. (2008) Kinetics of Non-catalyzed Decomposition of Glucose in High-temperature Liquid Water. Chin. J. Chem. Eng. 16 (6), 890-894.

(11) Soussy, S., El-Hellani, A., Baalbaki, R., Salman, R., Shihadeh, A., and Saliba, N. A. (2016) Detection of 5-hydroxymethylfurfural and furfural in the aerosol of electronic cigarettes. Tob Control 25, No. ii88. 
(12) Briganti, M., Delnevo, C. D., Brown, L., Hastings, S. E., and Steinberg, M. B. (2019) Bibliometric Analysis of Electronic Cigarette Publications: 2003(-)2018. Int. J. Environ. Res. Public Health 16 (3), 320.

(13) Krishnan-Sarin, S., Jackson, A., Morean, M., Kong, G., Bold, K. W., Camenga, D. R., Cavallo, D. A., Simon, P., and Wu, R. (2019) E-cigarette devices used by high-school youth. Drug Alcohol Depend. 194, 395-400.

(14) Burdock, G. A., and Fenaroli, G. Fenaroli's handbook of flavor ingredients, 6th ed; CRC Press/Taylor \& Francis Group: Boca Raton, 2010.

(15) Hammond, D., Wackowski, O. A., Reid, J. L., and O’Connor, R. J. (2018) International Tobacco Control Policy Evaluation Project, Use of Juul E-Cigarettes Among Youth in the United States. Nicotine Tob Res., nty237.

(16) Wiet, S. G., and Beyts, P. K. (1992) Sensory Characteristics of Sucralose and Other High-Intensity Sweeteners. J. Food Sci. 57 (4), 1014-1019.

(17) Rosbrook, K., Erythropel, H. C., DeWinter, T. M., Falinski, M., O’Malley, S., Krishnan-Sarin, S., Anastas, P. T., Zimmerman, J. B., and Green, B. G. (2017) The effect of sucralose on flavor sweetness in electronic cigarettes varies between delivery devices. PLoS One 12 (10), e0185334.

(18) Grotz, V. L., and Munro, I. C. (2009) An overview of the safety of sucralose. Regul. Toxicol. Pharmacol. 55 (1), 1-5.

(19) de Oliveira, D. N., de Menezes, M., and Catharino, R. R. (2015) Thermal degradation of sucralose: a combination of analytical methods to determine stability and chlorinated byproducts. Sci. Rep. 5, 9598.

(20) Rahn, A., and Yaylayan, V. A. (2010) Thermal degradation of sucralose and its potential in generating chloropropanols in the presence of glycerol. Food Chem. 118 (1), $56-61$.

(21) Duell, A. K., Pankow, J. F., Gillette, S. M., and Peyton, D. H. (2018) Boiling points of the propylene glycol + glycerol system at 1 atm pressure: $188.6-292^{\circ} \mathrm{C}$ without and with added water or nicotine. Chem. Eng. Commun. 205, 1691.

(22) Jensen, R. P., Luo, W., Pankow, J. F., Strongin, R. M., and Peyton, D. H. (2015) Hidden formaldehyde in e-cigarette aerosols. N. Engl. J. Med. 372 (4), 392-4.

(23) Jensen, R. P., Strongin, R. M., and Peyton, D. H. (2017) Solvent Chemistry in the Electronic Cigarette Reaction Vessel. Sci. Rep. 7, 42549.

(24) Salamanca, J. C., Meehan-Atrash, J., Vreeke, S., Escobedo, J. O., Peyton, D. H., and Strongin, R. M. (2018) E-cigarettes can emit formaldehyde at high levels under conditions that have been reported to be non-averse to users. Sci. Rep. 8 (1), 7559.

(25) Salamanca, J. C., Munhenzva, I., Escobedo, J. O., Jensen, R. P., Shaw, A., Campbell, R., Luo, W., Peyton, D. H., and Strongin, R. M. (2017) Formaldehyde 
Hemiacetal Sampling, Recovery, and Quantification from Electronic Cigarette Aerosols. Sci. Rep. 7 (1), 11044.

(26) Erythropel, H. C., Jabba, S. V., DeWinter, T. M., Mendizabal, M., Anastas, P. T., Jordt, S. E., and Zimmerman, J. B., Formation of flavorant-propylene Glycol Adducts With Novel Toxicological Properties in Chemically Unstable E-Cigarette Liquids. Nicotine Tob. Res. 2018. DOI: 10.1093/ntr/nty192

(27) de Saint Laumer, J. Y., Cicchetti, E., Merle, P., Egger, J., and Chaintreau, A. (2010) Quantification in gas chromatography: prediction of flame ionization detector response factors from combustion enthalpies and molecular structures. Anal. Chem. 82 (15), 6457-62.

(28) Duell, A. K., Pankow, J. F., and Peyton, D. H. (2018) Free-Base Nicotine Determination in Electronic Cigarette Liquids by ${ }^{1} \mathrm{H}$ NMR Spectroscopy. Chem. Res. Toxicol. 31 (6), 431-434.

(29) CORESTA Routine Analytical Machine for E-Cigarette Aerosol Generation and Collection - Definitions and Standard Conditions. https://www.coresta.org/routineanalytical-machine-e-cigaretteaerosol-generation-and-collection-definitions-and-standard (accessed 03/22/2018).

(30) Beauval, N., Antherieu, S., Soyez, M., Gengler, N., Grova, N., Howsam, M., Hardy, E. M., Fischer, M., Appenzeller, B. M. R., Goossens, J. F., Allorge, D., Garcon, G., LoGuidice, J. M., and Garat, A. (2017) Chemical Evaluation of Electronic Cigarettes: Multicomponent Analysis of Liquid Refills and their Corresponding Aerosols. J. Anal. Toxicol. 41 (8), 670-678.

(31) Beauval, N., Verriele, M., Garat, A., Fronval, I., Dusautoir, R., Antherieu, S., Garcon, G., Lo-Guidice, J. M., Allorge, D., and Locoge, N. (2019) Influence of puffing conditions on the carbonyl composition of e-cigarette aerosols. Int. J. Hyg. Environ. Health 222, 136.

(32) Pankow, J. F., Kim, K., McWhirter, K. J., Luo, W., Escobedo, J. O., Strongin, R. M., Duell, A. K., and Peyton, D. H. (2017) Benzene formation in electronic cigarettes. PLoS One 12 (3), No. e0173055.

(33) Grice, H. C., and Goldsmith, L. A. (2000) Sucralose-an overview of the toxicity data. Food Chem. Toxicol. 38, S1-6.

(34) Jang, M., Czoschke, N. M., Lee, S., and Kamens, R. M. (2002) Heterogeneous atmospheric aerosol production by acid-catalyzed particle-phase reactions. Science 298 (5594), 814-7.

(35) Pankow, J. F. (2017) Calculating compound dependent gasdroplet distributions in aerosols of propylene glycol and glycerol from electronic cigarettes. J. Aerosol Sci. 107,9-13. 
(36) European Commission, S. C. o. F. Opinion of the Scientific Committee on Food on 3-monochloro-propande-1,2-diol (3-MCPD); Rue de la Loi 200, B-1049

Bruxelles/Wetstraat 200, B-1049 Brussel Belgium, 2001.

(37) Lin, Y. C., Cho, J., Tompsett, G. A., Westmoreland, P. R., and Huber, G. W. (2009) Kinetics and Mechanism of Cellulose Pyrolysis. J. Phys. Chem. C 113 (46), 20097-20107.

(38) Meng, X., Zhang, H. Y., Liu, C., and Xiao, R. (2016) Comparison of Acids and Sulfates for Producing Levoglucosan and Levoglucosenone by Selective Catalytic Fast Pyrolysis of Cellulose Using Py-GC/MS. Energy Fuels 30 (10), 8369-8376.

(39) Farsalinos, K. E., Voudris, V., and Poulas, K. (2015) Ecigarettes generate high levels of aldehydes only in 'dry puff' conditions. Addiction 110 (8), 1352-6.

(40) Benowitz, N. L. (2010) Nicotine addiction. N. Engl. J. Med. 362 (24), 2295-303.

(41) Koob, G. F., and Le Moal, M. (2001) Drug addiction, dysregulation of reward, and allostasis. Neuropsychopharmacology 24 (2), 97-129.

(42) Andre, E., Campi, B., Materazzi, S., Trevisani, M., Amadesi, S., Massi, D., Creminon, C., Vaksman, N., Nassini, R., Civelli, M., Baraldi, P. G., Poole, D. P., Bunnett, N. W., Geppetti, P., and Patacchini, R. (2008) Cigarette smoke-induced neurogenic inflammation is mediated by alpha, beta-unsaturated aldehydes and the TRPA1 receptor in rodents. J. Clin. Invest. 118 (7), 2574-82.

(43) Birrell, M. A., Belvisi, M. G., Grace, M., Sadofsky, L., Faruqi, S., Hele, D. J., Maher, S. A., Freund-Michel, V., and Morice, A. H. (2009) TRPA1 agonists evoke coughing in guinea pig and human volunteers. Am. J. Respir. Crit. Care Med. 180 (11), $1042-7$.

(44) McNamara, C. R., Mandel-Brehm, J., Bautista, D. M., Siemens, J., Deranian, K. L., Zhao, M., Hayward, N. J., Chong, J. A., Julius, D., Moran, M. M., and Fanger, C. M. (2007) TRPA1 mediates formalininduced pain. Proc. Natl. Acad. Sci. U. S. A. 104 (33), 13525-30.

(45) Talavera, K., Gees, M., Karashima, Y., Meseguer, V. M., Vanoirbeek, J. A., Damann, N., Everaerts, W., Benoit, M., Janssens, A., Vennekens, R., Viana, F., Nemery, B., Nilius, B., and Voets, T. (2009) Nicotine activates the chemosensory cation channel TRPA1. Nat. Neurosci. 12 (10), 1293-9.

(46) Willis, D. N., Liu, B., Ha, M. A., Jordt, S. E., and Morris, J. B. (2011) Menthol attenuates respiratory irritation responses to multiple cigarette smoke irritants. FASEB J. 25 (12), 4434-44.

(47) Carstens, E., Kuenzler, N., and Handwerker, H. O. (1998) Activation of neurons in rat trigeminal subnucleus caudalis by different irritant chemicals applied to oral or ocular mucosa. J. Neurophysiol. 80 (2), 465-92. 
(48) Talhout, R., Opperhuizen, A., and van Amsterdam, J. G. (2007) Role of acetaldehyde in tobacco smoke addiction. Eur. Neuropsychopharmacol. 17 (10), 627-36. 
3.11. Supporting Information: Sucralose-enhanced Degradation of Electronic Cigarette Liquids During Vaping

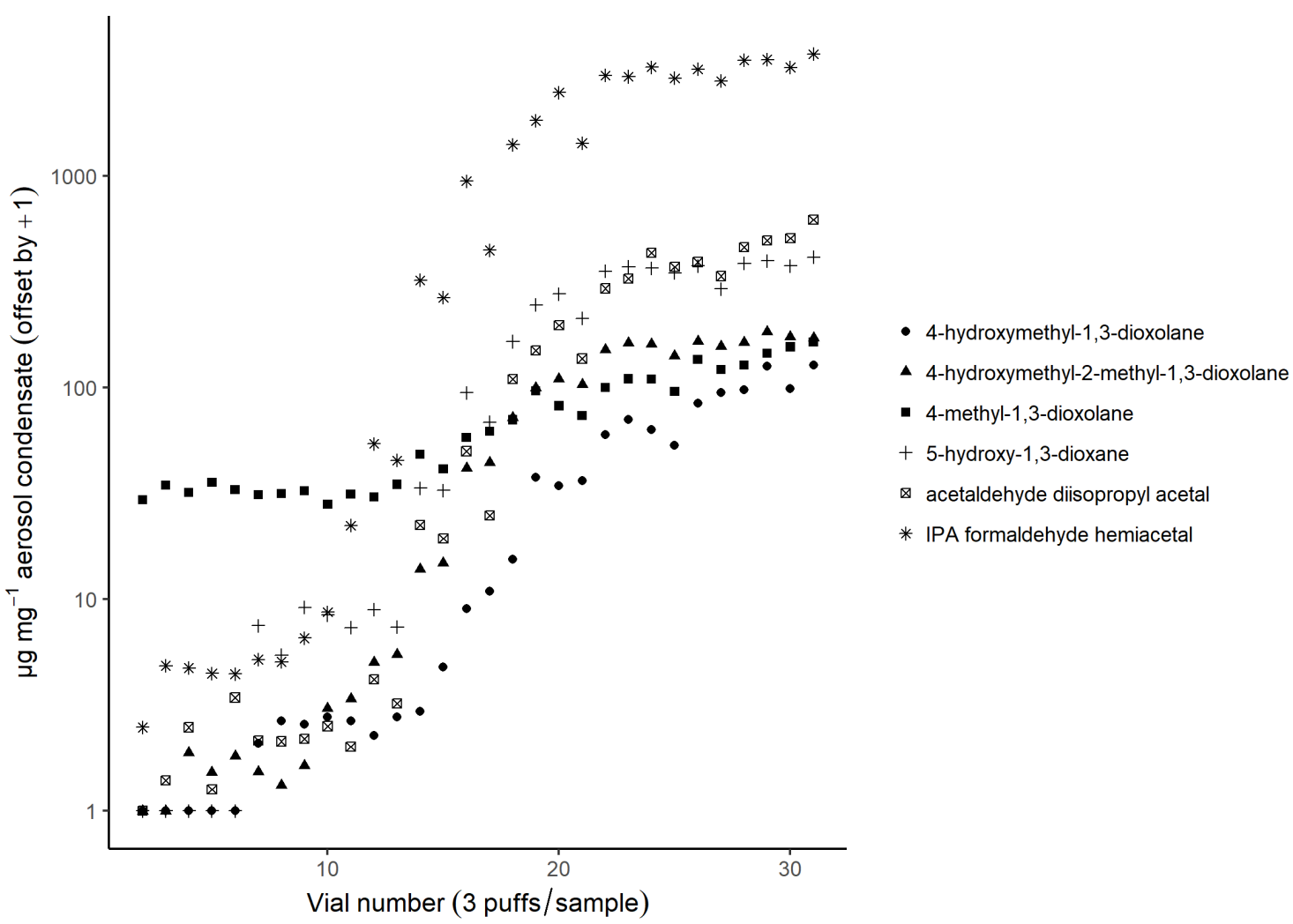

Figure $\mathrm{S} 1: \mu \mathrm{g}$ acetals and hemiacetal per $\mathrm{mg}$ aerosol condensate detected in each vial as detected by GC/MS or GC/FID generated using a $1.2 \Omega$ coil at 20 watts containing 1:1 molar propylene glycol and glycerol.

Values of 1 represent compounds not detected as zero cannot be represented on a log scale. Cyclic acetals could go undetected with DNPH-derivatization detection methods. IPA hemiformal, higher than other formaldehyde equivalent analytes by a factor of $\sim 10$, represents free formaldehyde (less likely as gas phase was not collected) or propylene glycol (PG) and/or glycerol (GL) hemiacetals (more likely) captured in the aerosol and formed after addition of isopropanol to sample vials via exchange with the 
more abundant isopropanol. All acetals increase over the course of the puffing regime. Conditioning puffs were not employed in this sampling regime which highlights that toxicant levels may be underestimated if a limited number of puffs on a new atomizer are used.

\subsubsection{Isopropanol Hemiformal Determination}

The hemiacetal of isopropanol and formaldehyde is not stable therefore an authentic chemical standard is not available for purchase. A mixture containing isopropanol hemiformal was prepared by bubbling nitrogen gas into a vial containing a formaldehyde-enriched liquid solution via capillary tube. Another capillary tube was positioned in the headspace above the liquid level of formaldehyde solution and routed under the liquid level of another vial containing isopropanol. A capillary tube open to atmosphere was positioned in the isopropanol containing vial to allow release of excess gas. Gas from the headspace of the formaldehyde solution vial was allowed to bubble through isopropanol for approximately 30 minutes. The isopropanol solution was analyzed by GC/MS in both electron ionization (EI) and chemical ionization (CI). Retention time and spectra of the generated isopropanol hemiformal matched that of the suspected peak found in samples. The peak was not replicated when the above experiment was carried out substituting acetone for isopropanol. The suspected isopropanol hemiformal was observed to be stable at room temperature for weeks (as indicated by multiple injections over this time) along with hemiacetals of isopropanol and formaldehyde oligomers which were also identified by ${ }^{1} \mathrm{H}$ NMR testing of an aliquot the isopropanol solution. 


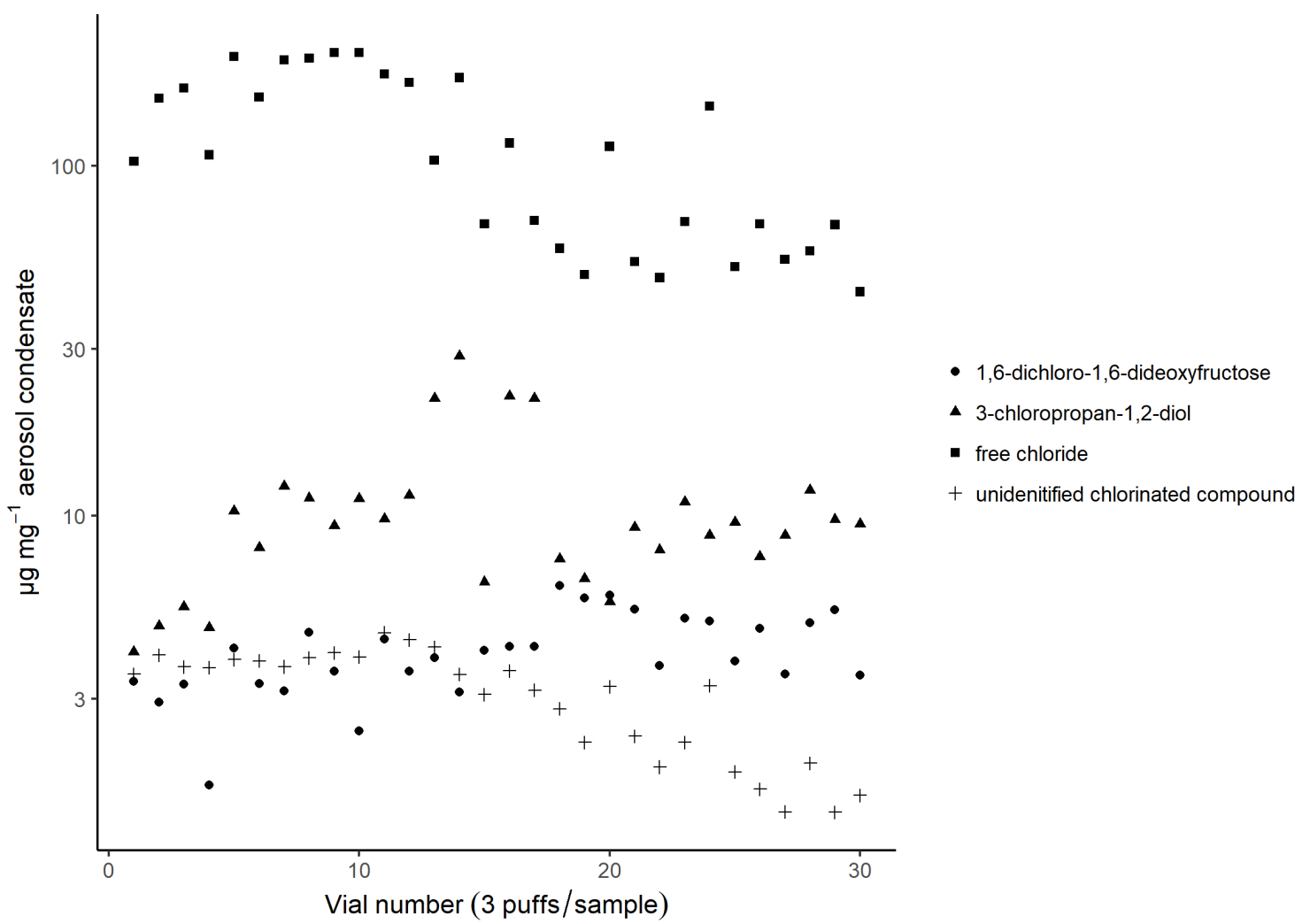

Figure S2: Chlorinated targets detected in each vaporized sample vial as detected by $\mathrm{GC} / \mathrm{MS}, \mathrm{GC} / \mathrm{FID}$, or IC generated using a $1.2 \Omega$ coil at 20 watts containing 1:1 molar PG and GL.

\subsubsection{Comparison of NMR Degradation Data with the Literature}

Variability between individual experiments makes it difficult to readily compare degradation levels between this work and other studies reported in the literature. The coil resistance, wattage employed, device type, wicking material, puff duration, collection method, analysis method, etc. can all influence the resulting values. ${ }^{1}$ Below are two studies that attempt to suggest how much degradation can be produced by e-cigarettes.

In this study, the amount of each degradation component was compared to the quantity of (PG) in each vaporized sample. The NMR data was used to calculate 
integration values for each component relative to the amount of PG in the sample, as found from its -CH3 at $1.05 \mathrm{ppm}$ (Figure S3). The LOD (as defined as the standard deviation of the noise X 3) and the LOQ (as defined as the standard deviation of the noise $\mathrm{X} 10$ ) were $0.006 \%$ and $0.02 \%$ of the PG integration value, respectively.

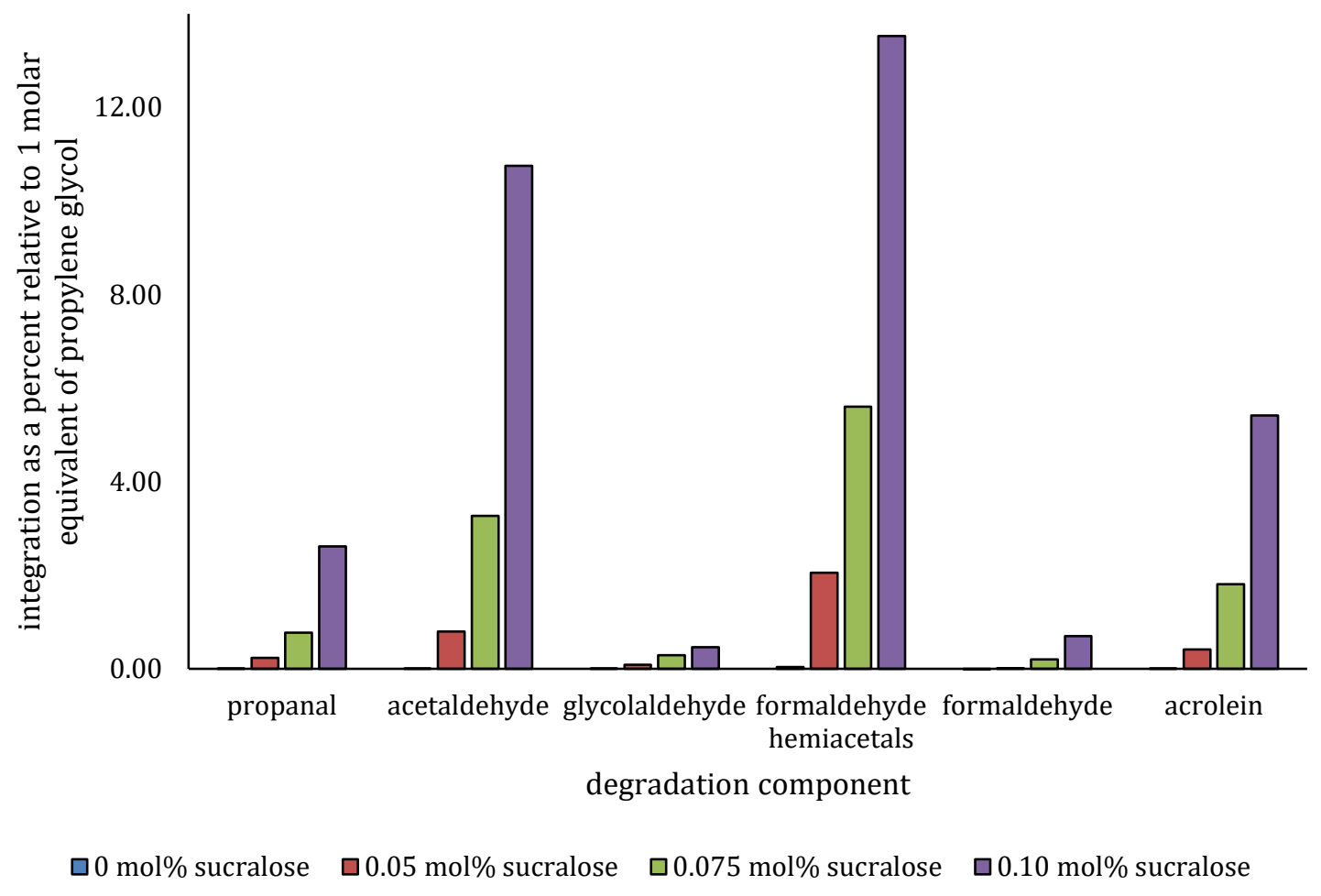

Figure S3: NMR integration values as a percent of the propylene glycol in each sample.

Salamanca et al. (2017) ${ }^{1}$ reported the yields for formaldehyde and PG and/or GL + formaldehyde hemiacetals ("formaldehyde hemiacetals") at both 10 and 15 watts using a different resistance coil (2.2 $\Omega$ ), e-cigarette device (KangerTech ProTank II atomizer), and collection method (DNPH collection method combined with qNMR analysis). Samples generated at 10 watts resulted in $1.20 \mu \mathrm{g}$ of formaldehyde per mg e-liquid 
consumed and $16.75 \mu \mathrm{g}$ of formaldehyde hemiacetals per mg e-liquid consumed. ${ }^{1}$ At 15 watts, $4.43 \mu \mathrm{g}$ formaldehyde and $65.70 \mu \mathrm{g}$ formaldehyde hemiacetals were reported. ${ }^{1}$

To compare our values to those reported by Salamanca et al., ${ }^{1}$ the change in the mass of the vial (after collecting the sample into it) was used to calculate the mass of each component based on the molar ratios (as determined by NMR integration) and using the molecular weight of each component (Figure S4). Only PG, GL, propanal, acetaldehyde, glycolaldehyde, formaldehyde hemiacetals, formaldehyde, and acrolein were accounted for by NMR integration in this analysis. This means that values could be overestimated due to ignoring other components. Water, which generally comprises a significant portion of the vaped sample was not accounted for; this is a limitation. Other unknown degradation components were also excluded from this analysis. The change in the mass of the tank was used to calculate the volume of e-liquid consumed (in $\mathrm{mL}$ ) in order to convert these degradation masses overall to $\mu \mathrm{g}$ degradation per $\mathrm{mL}$ of e-liquid consumed (also ignoring water in the starting e-liquid). Another limitation of this is that only a fraction of the total aerosol produced (as measured by the $\mathrm{mL}$ e-liquid consumed) was captured in the vial and tested by NMR. This may mean that the degradation values presented herein are an underestimation of the total quantity of degradation that was produced.

Furthermore, the PG and/or GL formaldehyde hemiacetals appear in the same region of the spectrum, and it is difficult to separate the PG and GL forms for analysis. Due to this, the masses of formaldehyde hemiacetals were approximated by using the molecular weight of the PG + formaldehyde hemiacetal. There was also a delay between 
the acquisition of the original spectrum (where the aldehyde degradation products were analyzed) and the addition of sodium bicarbonate so that the formaldehyde hemiacetals could be visualized. In solution, formaldehyde can continue to react with PG and GL and form more hemiacetals over time. Due to this, the quantitation of total formaldehyde products (in this case free formaldehyde and the formaldehyde hemiacetals) is likely overestimated due to the ongoing formation of the formaldehyde hemiacetals until there is no free formaldehyde remaining. The LOD and LOQ for these values in Figure S4 were 7 and $22 \mu \mathrm{g}$ per $\mathrm{mL}$ of e-liquid vaped, respectively.

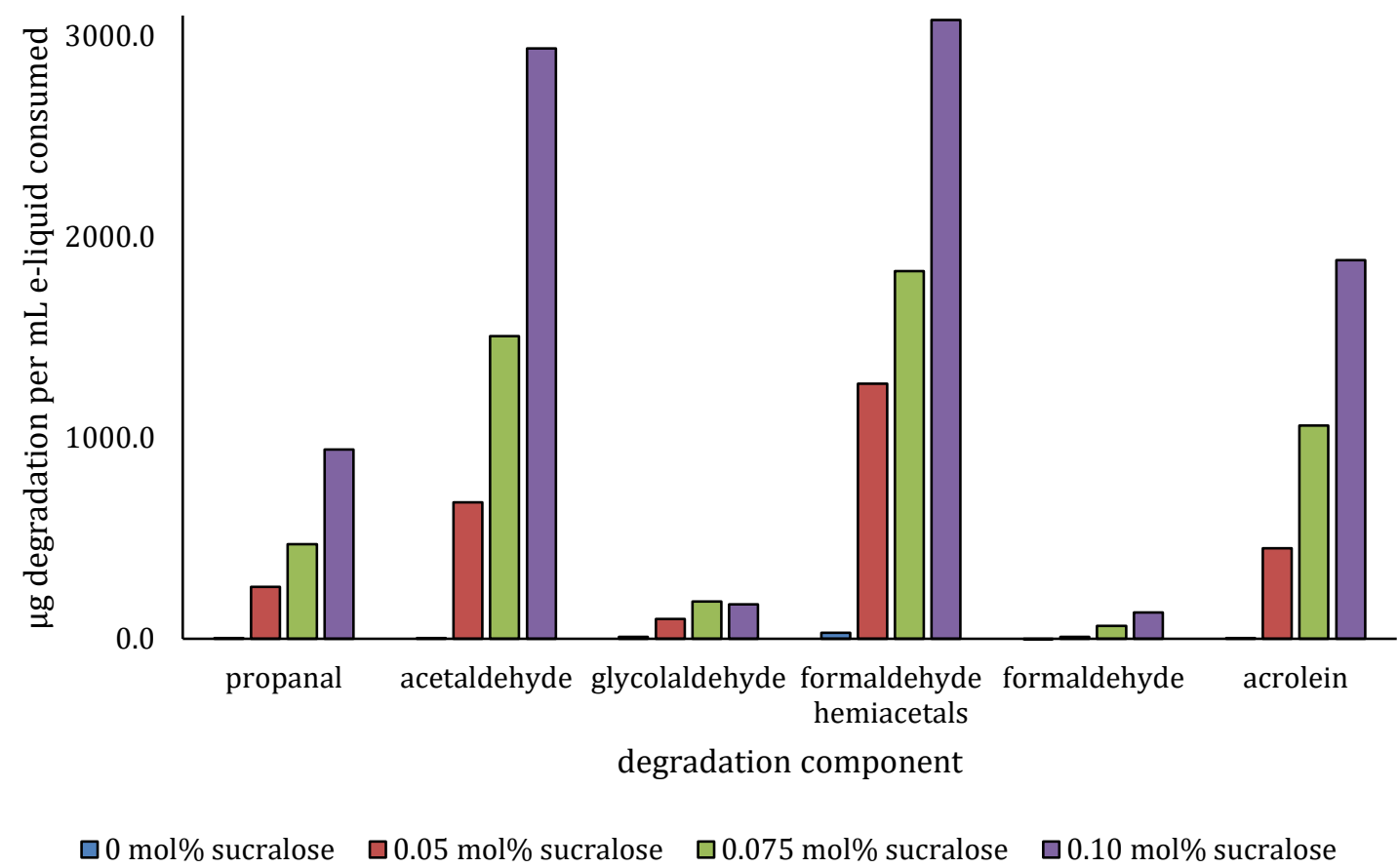

Figure S4: $\mu \mathrm{g}$ of each degradation component per $\mathrm{mL}$ of e-liquid consumed calculated from NMR integration values.

Geiss et al. (2016) ${ }^{2}$ provided an overview of existing degradation concentrations, associated methods employed, e-cigarette device, and e-liquid. Of the studies included by 
Geiss et al., the highest concentrations of formaldehyde, acetaldehyde, and acrolein from all studies are as follows: 3400,2600 , and $2500 \mathrm{ng} / \mathrm{puff}$.

To convert our values to the same units as those used by Geiss et al., the mass values for each degradation component (calculated based on the molar ratios and the trapped sample mass) that were used to calculate the $\mu \mathrm{g}$ of each degradation component per $\mathrm{mL}$ of e-liquid consumed in Figure S4 were converted to ng and divided by 3 since each sample was composed of 3 puffs. The LOD and LOQ for the values in Figure S5 were 147 and $488 \mathrm{ng} /$ puff, respectively.

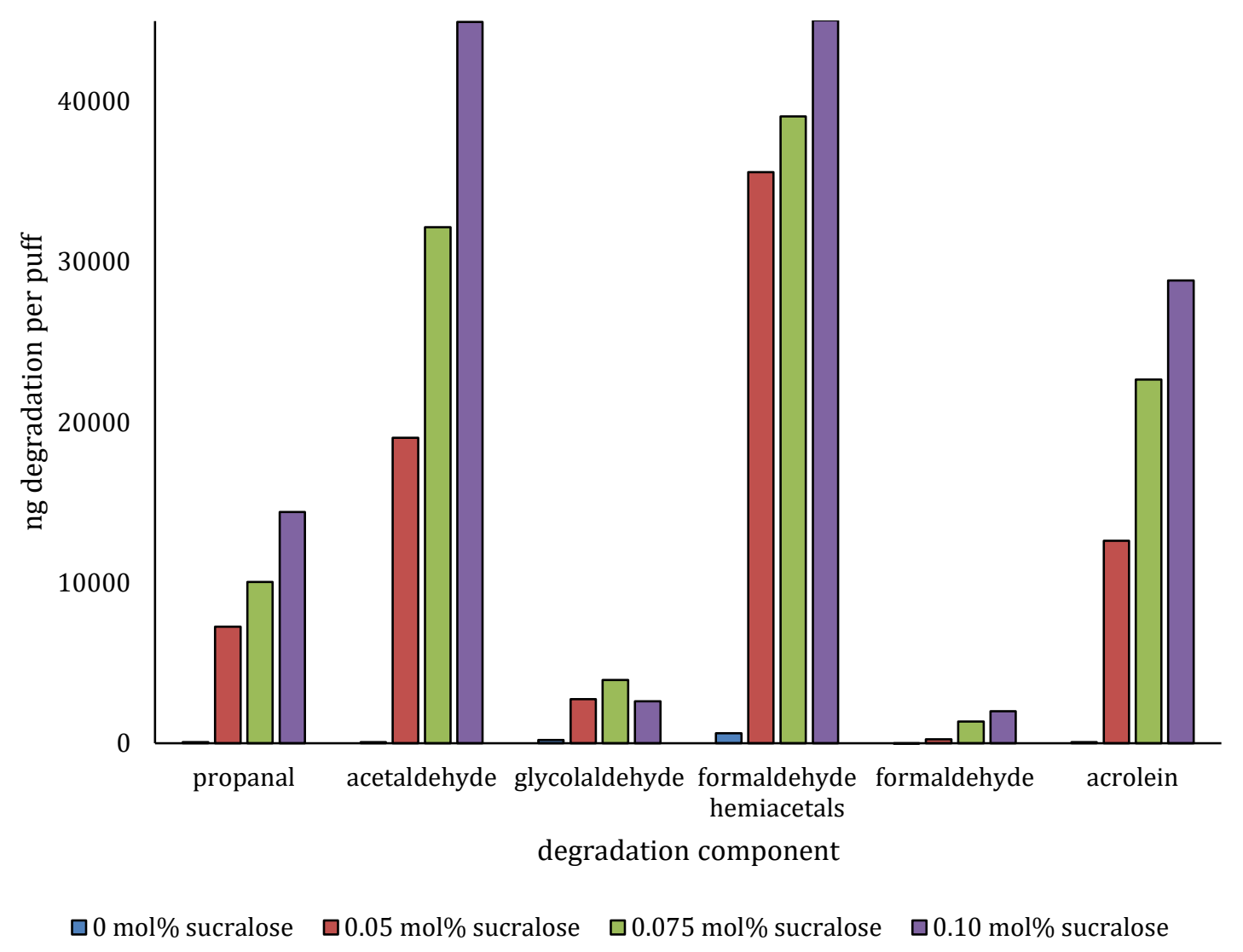

Figure S5: ng of each degradation component per puff. 


\subsubsection{Supplemental Material References}

1. Salamanca, J. C.; Munhenzva, I.; Escobedo, J. O.; Jensen, R. P.; Shaw, A.; Campbell, R.; Luo, W.; Peyton, D. H.; Strongin, R. M., Formaldehyde Hemiacetal Sampling, Recovery, and Quantification from Electronic Cigarette Aerosols. Sci Rep 2017, 7 (1), 11044.

2. Geiss, O.; Bianchi, I.; Barrero-Moreno, J., Correlation of volatile carbonyl yields emitted by e-cigarettes with the temperature of the heating coil and the perceived sensorial quality of the generated vapours. Int J Hyg Environ Health 2016, 219 (3), 26877. 
4. Free-Base Nicotine Determination in Electronic Cigarette Liquids by ${ }^{1} \mathrm{H}$ NMR Spectroscopy

Anna K. Duell, $\dagger$ James F. Pankow, $₫$ and David H. Peyton*,$\dagger$

'Department of Chemistry and Department of Engineering, Portland State University, Portland, Oregon 97207-0751, United States

Reprinted (adapted) with permission from:

Duell, Anna K., James F. Pankow, and David H. Peyton. "Free-base nicotine determination in electronic cigarette liquids by ${ }^{1} \mathrm{H}$ NMR spectroscopy." Chemical research in toxicology 31.6 (2018): 431-434.

Copyright 2018 American Chemical Society. Further permissions related to the material excerpted should be directed to the ACS.

Link to source: https://pubs.acs.org/doi/10.1021/acs.chemrestox.8b00097 


\subsection{Abstract}

E-liquids usually contain significant nicotine, which will exist primarily in two forms, monoprotonated and free-base, the proportions of which are alterable through the effective $\mathrm{pH}$ of the medium. The fraction of nicotine in the free-base form is $\alpha_{\mathrm{fb}}$, with $0 \leq$ $\alpha_{\mathrm{fb}} \leq 1$. When dosed via aerosol, the two nicotine forms have different mechanisms and kinetics of delivery, as well as differing implications for harshness of the inhaled aerosol, so $\alpha_{\mathrm{fb}}$ is relevant regarding abuse liability. Previous attempts to determine $\alpha_{\mathrm{fb}}$ in electronic cigarette liquids and vapor have been flawed. We employed the exchangeaveraged ${ }^{1} \mathrm{H}$ NMR chemical shifts of nicotine to determine $\alpha_{\mathrm{fb}}$ in samples of e-liquids. This method is rapid and direct and can also be used with collected aerosol material. The e-liquids tested were found to have $0.03 \leq \alpha_{\mathrm{fb}} \leq 0.84$. The $\alpha_{\mathrm{fb}}$ values in collected aerosol liquid samples were highly correlated with those for the parent e-liquids. E-liquids designed to combine high total nicotine level (addictive delivery) with low $\alpha_{\mathrm{fb}}$ (for ease of inhalation) are likely to be particularly problematic for public health. 


\subsection{Main Text}

In the United States during 2016, electronic cigarettes (e-cigarettes) were used regularly by $\sim 8$ million adults. ${ }^{1,2}$ For high school students, CDC surveys estimate ecigarette use in 2013, 2014, 2015, and 2016 to have been 5, 13, 16, and 11\%, respectively, and for conventional cigarettes $13,9,9$, and $8 \%$, respectively. ${ }^{2,3}$ Often $\operatorname{argued}^{4}$ though not proven to be safer than conventional cigarettes, ${ }^{5,6}$ e-cigarettes are not, in any case, risk free. And, many e-cigarette liquids (e-liquids) contain substantial nicotine, which is addictive and can be toxic.

Nicotine has three forms: free-base (Nic, aka unprotonated), monoprotonated $\left(\mathrm{NicH}^{+}\right)$, and diprotonated $\left(\mathrm{NicH}_{2}{ }^{2+}\right)$. The protonation state of nicotine can be altered by changing the acid/base conditions in the medium. ${ }^{7,8}$ In water at $25^{\circ} \mathrm{C}, \mathrm{p} K_{1}$ (for $\mathrm{NicH}_{2}{ }^{2+}$ ) and $\mathrm{p} K_{2}\left(\right.$ for $\mathrm{NicH}^{+}$) are 3.10 and 8.01, respectively. ${ }^{9}$ Tobacco smoke aerosols are believed to contain primarily the $\mathrm{Nic}$ and $\mathrm{NicH}^{+}$forms (Figure 1) because conditions in the aerosol particulate material (PM) are not considered to be sufficiently acidic to generate significant $\mathrm{NicH}_{2}{ }^{2+} \cdot 7,8$

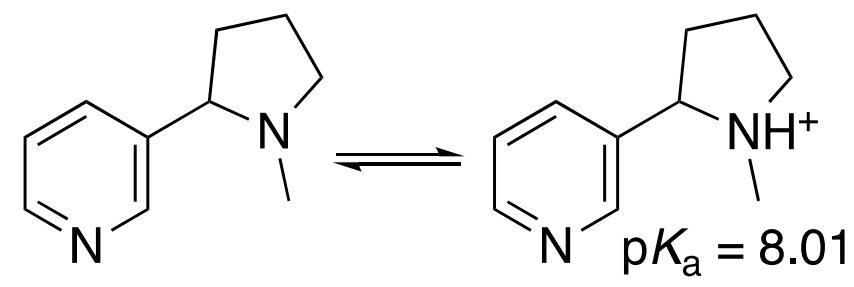

Figure 1. The distribution of nicotine in tobacco and vape aerosols primarily involves two forms; left) Nic (free-base) which has volatility; and right) $\mathrm{NicH}^{+}$(monoprotonated) which is non-volatile. The fraction of the free-base for $\left(\alpha_{\mathrm{fb}}\right)$ depends on the acid/base conditions. 
The fraction of nicotine in the free-base form is $\alpha_{\mathrm{fb}}$, with $0 \leq \alpha_{\mathrm{fb}} \leq 1$ :

$$
\alpha_{\mathrm{fb}} \equiv \frac{[\mathrm{Nic}]}{[\mathrm{Nic}]+\left[\mathrm{NicH}^{+}\right]}
$$

where $\mathrm{NicH}_{2}{ }^{2+}$ is neglected. The $\alpha_{\mathrm{fb}}$ can affect the kinetics and location of nicotine uptake from an inhaled aerosol because the free-base form is volatile: it can deposit from an inhaled tobacco smoke (or vape) aerosol from the gas phase and by particle deposition, whereas only particle deposition is operative for protonated nicotine. ${ }^{10}$ It has been argued that these considerations make it likely that $\alpha_{\mathrm{fb}}$ affects nicotine addiction potential. ${ }^{11,12}$ In addition, high $\alpha_{\mathrm{fb}}$ values have long been connected with tobacco smoke harshness upon inhalation. $^{13}$

In water, neglecting $\mathrm{NicH}_{2}{ }^{2+}$

$$
\alpha_{\mathrm{fb}}=\frac{1}{1+10^{-\mathrm{pH}} / K_{a}}
$$

where $K_{\mathrm{a}}$ is the acidity constant for $\mathrm{NicH}^{+}$in water ( $K_{2}$ as given above). Other than nicotine level, commercial labels on e-liquid products currently provide little compositional information, and these labels certainly do not indicate $\alpha_{\mathrm{fb}}$ values.

Historically, methods for determination of $\alpha_{\mathrm{fb}}$ in tobacco smoke PM have been flawed. ${ }^{10}$ One method introduced a significant amount of water for subsequent measurement of the $\mathrm{pH}$ of the aqueous phase, ${ }^{14}$ and a second introduced water and an organic solvent (e.g., chloroform) for what was intended to be a selective extraction of the neutral free-base form. ${ }^{15}$ Given the disrupting effects of added liquids, neither method can give good results. Pankow et al. ${ }^{16}$ describe a successful method for $\alpha_{\mathrm{fb}}$ determination 
in tobacco smoke PM that uses equilibration with a gas volume as a means to detect volatile nicotine, which is taken to be proportional to $\alpha_{\mathrm{fb}}$. In addition, direct measurement by ${ }^{1} \mathrm{H}$ NMR spectroscopy of $\alpha_{\mathrm{fb}}$ is possible for tobacco smoke $\mathrm{PM}^{17}$ and for PM from the now-defunct Eclipse product ${ }^{7}$ which gave aerosols compositionally similar to those from e-liquids. (Others attempted using NMR, but added a solvent that will perturb $\alpha_{\mathrm{fb}}{ }^{18}$ ) Our work reported here describes the development of ${ }^{1} \mathrm{H}$ NMR spectroscopy for measurement of $\alpha_{\mathrm{fb}}$ in e-liquids and their aerosols. The materials and methods are provided in the Supporting Information.

For each sample, nicotine ${ }^{1} \mathrm{H}$ chemical shifts $(\delta)$ were measured for different protons on the nicotine molecule $\left(\mathrm{H}_{\mathrm{a}}\right.$ through $\left.\mathrm{H}_{\mathrm{e}}\right)$. The assignments are in accordance with those previously made ${ }^{17}$ and verified by the J-coupling patterns and integrations. The $\delta$ of $\mathrm{H}_{\mathrm{e}}$ was subtracted from $\mathrm{H}_{\mathrm{a}}$ through $\mathrm{H}_{\mathrm{d}}$ to obtain the difference, $\Delta \delta$, as in eq 3 , noting that $\Delta \delta$ depends on its position in the molecule, that is, some of the protons shift more than others.

$$
\Delta \delta=\left[\delta \mathrm{H}_{\text {aromatic proton }}\left(\text { i. e. , } \mathrm{H}_{\mathrm{a}} \text { through } \mathrm{H}_{\mathrm{d}}\right)\right]-\left[\delta \mathrm{H}_{\mathrm{e}}\right]
$$

Nicotine standards (24 mg nicotine / mL in PG/GL mixtures; see Supporting Information) were then used to calculate $\Delta \delta$ for the monoprotonated and free-base states of nicotine after assessment with a variety of acids and concentrations thereof. In practice, we used only the aromatic protons $\mathrm{H}_{\mathrm{a}}$ and $\mathrm{H}_{\mathrm{b}}$ to avoid steric or direct charge contributions that may affect the chemical shifts of $\mathrm{H}_{\mathrm{c}}$ and $\mathrm{H}_{\mathrm{d}}$; these protons being proximal to the nicotine pyrrolidine ring. Commercial e-liquid samples were then evaluated by the use of eq 4, with the resonances indicated in Figure $2:{ }^{17}$ 


$$
\alpha_{\mathrm{fb}}=\frac{\left[\left(\Delta \delta_{\text {commercial sample })}-\left(\Delta \delta_{\text {monoprotonated standard }}\right)\right]\right.}{\left[\left(\Delta \delta_{\text {commercial standard }}\right)-\left(\Delta \delta_{\text {monoprotonated standard })}\right]\right.}
$$

Thus, for "Taurus" (using the $\mathrm{H}_{\mathrm{a}}$ and $\mathrm{H}_{\mathrm{e}}$ chemical shifts):

$$
\alpha_{\mathrm{fb}}=\frac{[(6.120 \mathrm{ppm})-(5.942 \mathrm{ppm})]}{[(6.331 \mathrm{ppm})-(5.942 \mathrm{ppm})]}=0.46
$$

Free-base fractions $\left(\alpha_{\mathrm{fb}}\right)$ for a selection of commercial e-liquids were also calculated; the results are shown in Figure 3, with $\alpha_{\mathrm{fb}}$ ranging from 0.03 to 0.84 .

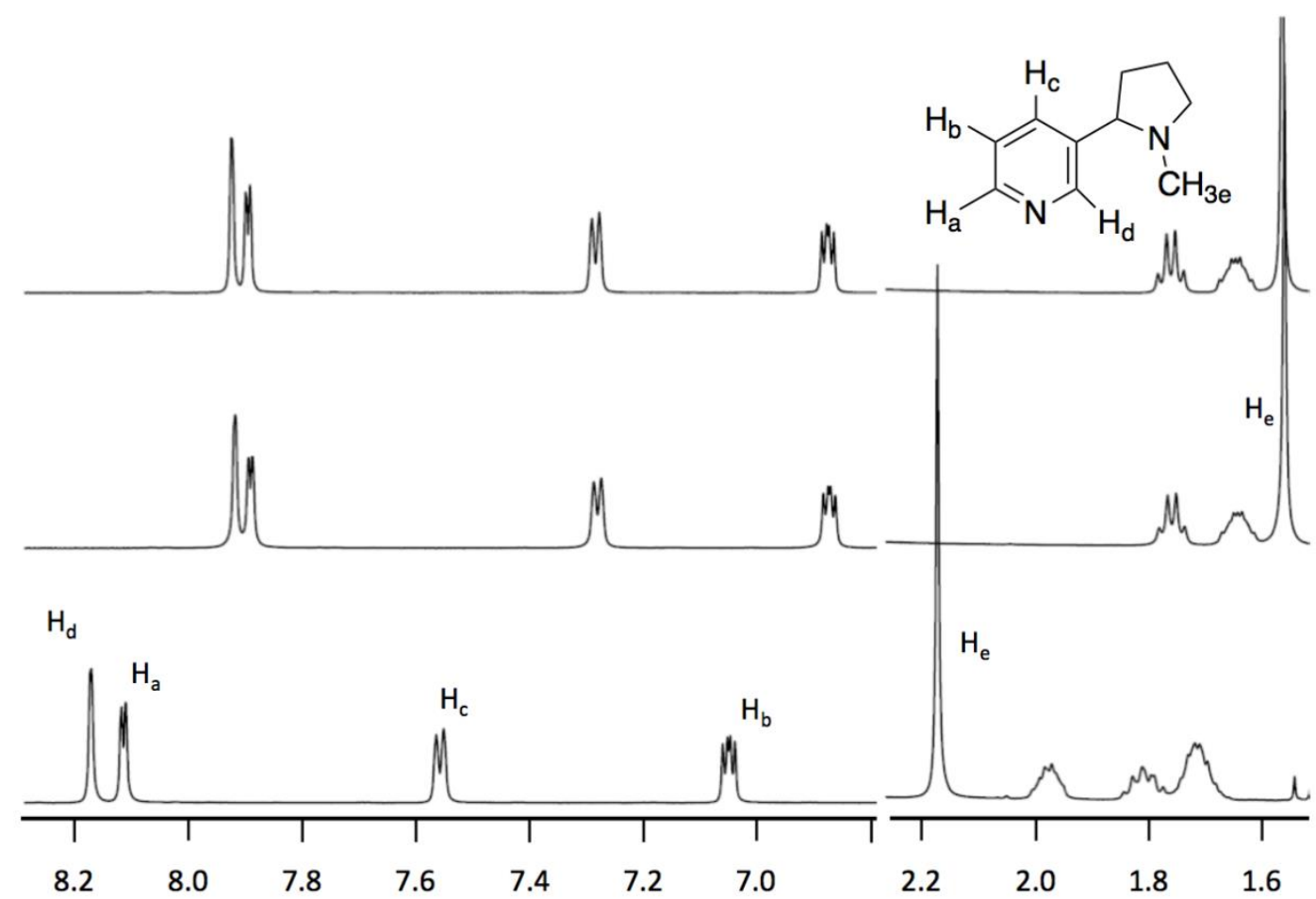

Figure 2. ${ }^{1} \mathrm{H}$ NMR spectra showing the chemical shifts changes for nicotine in a PG+GL stock mixture with the addition of acid and base, independently. Top to bottom: 1x tbutylamine added (relative to moles nicotine), PG+GL e-liquid stock (no acid or base additives), and 5x acetic acid added. Stock mixture contained 54 PG:46 GL (by moles) and $24 \mathrm{mg} / \mathrm{mL}$ nicotine. Samples were prepared by isolating the e-liquid sample in an inner concentric NMR tube, with DMSO- $d_{6}$ lock solvent in the outer tube, at $40^{\circ} \mathrm{C}$. 


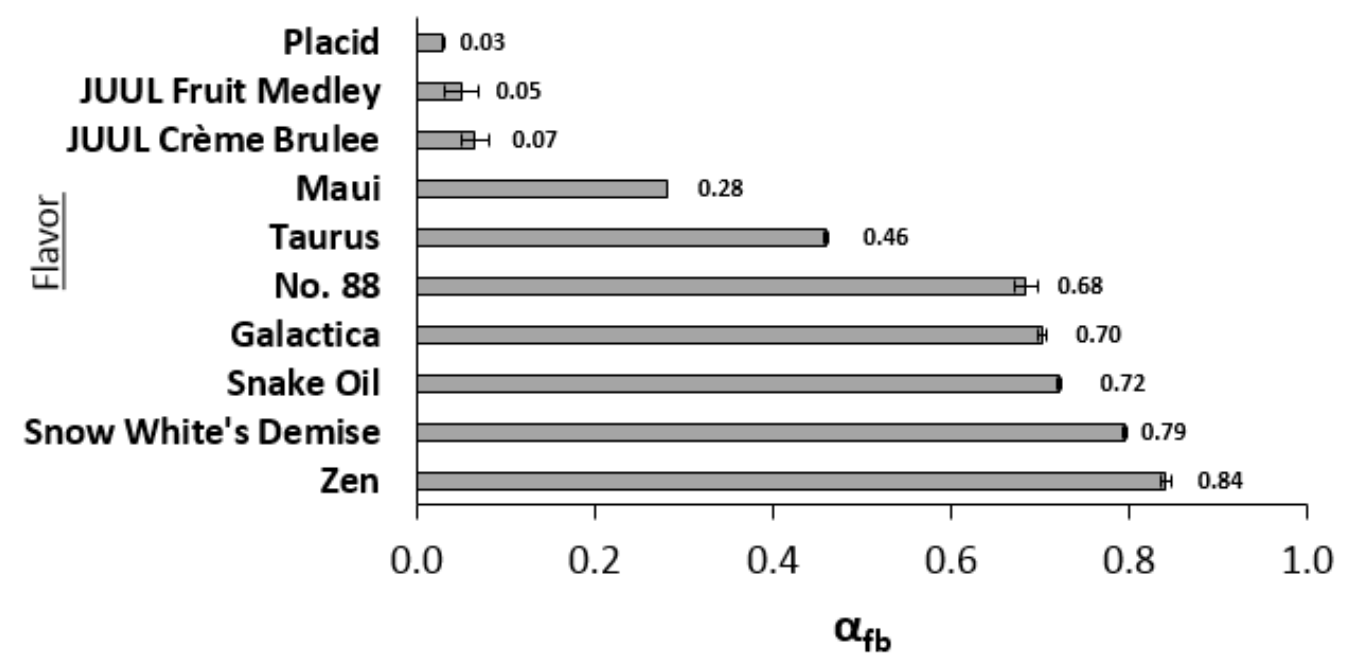

Figure 3. Free-base nicotine fraction $\left(\alpha_{\mathrm{fb}}\right)$ in commercial e-liquids as an average using aromatic protons $\mathrm{H}_{\mathrm{a}}$ and $\mathrm{H}_{\mathrm{b}}$. The ranges between chemical shift differences are indicated.

The accuracy of the method was verified by adding acid and base, respectively, to "Zen" flavored e-liquid aliquots. The resulting free-base and protonated direct chemical shift values were used to calculate $\alpha_{\mathrm{fb}}=0.83 \pm 0.00$ (range), which was statistically equal to the overall-calibration derived value of $0.84 \pm 0.01$ (range), using eq 4 as before. The accuracy of the method was verified by adding acid and base, respectively, to "Zen" flavored e-liquid aliquots. The resulting free-base and protonated direct chemical shift values were used to calculate $\alpha_{\mathrm{fb}}=0.83 \pm 0.00$ (range), which was statistically equal to the overall-calibration derived value of $0.84 \pm 0.01$ (range), using eq 4 as before.

As an initial examination of how vaporization may affect $\alpha_{\mathrm{fb}}$, e-liquids with high and low $\alpha_{\mathrm{fb}}$ values were vaporized, and the PM collected and analyzed. The "Zen" eliquid, which had the highest free-base content of the e-liquids tested, was found to have a post-vaporization $\alpha_{\mathrm{fb}}$ of $0.80 \pm 0.01$ (range), which is similar to the unvaporized value of $0.84 \pm 0.01$. "Maui" $(24 \mathrm{mg} / \mathrm{mL})$ was determined to have a post-vaporization $\alpha_{\mathrm{fb}}$ of $0.78 \pm 0.01$ (range), which is comparable to the unvaporized $\alpha_{\mathrm{fb}}$, which was $0.80 \pm 0.00$. 
The JUUL "crème brulee" flavored e-liquid was found to have a post-vaporization $\alpha_{\mathrm{fb}}$ of $0.05 \pm 0.03$ (range), also comparable to its unvaporized value of $0.07 \pm 0.02$. JUUL eliquids are advertised to contain benzoic acid, which we verified by NMR as being present primarily in its ionic, benzoate form.

The NMR method presented here may be compared with contemporary analogs for e-liquids of the two historical methods for $\alpha_{\mathrm{fb}}$ in tobacco smoke PM. First, Stepanov and Fujioka, ${ }^{19}$ Lisko et al., ${ }^{20}$ and El-Hellani et al. ${ }^{8}$ all describe diluting an aliquot of eliquid with water, measuring the $\mathrm{pH}$, and then calculating $\alpha_{\mathrm{fb}}$ by eq 2 . The result is that the values obtained suffer from both medium effects (water is different from an e-liquid) and dilution, though the $\mathrm{pH}$ values may, nevertheless, provide some useful relative indications of the overall acid/base balances in different e-liquids. However, that can be compromised if air-related $\mathrm{CO}_{2}$ is present in the added water and affects the measured $\mathrm{pH}$ values. This problem is likely evidenced in the data of Lisko et al. ${ }^{20}$ (see Supporting Information). Second, El-Hellani et al. ${ }^{8}$ describe making $6 \mathrm{~mL}$ aqueous solutions of eliquids, extracting with $6 \mathrm{~mL}$ toluene, and then determining nicotine in the toluene solvent extract as a measure of the nicotine percentage in the water. This approach suffers from the same dilution, medium, possible $\mathrm{CO}_{2}$ incursion effects discussed above and introduces uncertainties regarding the extent to which the toluene extraction step affects the position of the $\mathrm{NicH}^{+} \leftrightarrows \mathrm{Nic}+\mathrm{H}^{+}$equilibrium in the aqueous dilution.

In order to confirm the above concern directly, the JUUL "crème brulee" e-liquid was diluted into $\mathrm{D}_{2} \mathrm{O}$ to determine if $\alpha_{\mathrm{fb}}$ was affected by dilution into this deuterium 
analog of water. The dilution (5:1, by volume) was found to result in fully monoprotonated nicotine.

Although we used a $600 \mathrm{MHz}$ NMR system for this work, it is possible that these methods could be adapted for lower field NMR, and even benchtop instruments. This is a rapid and easy way to measure $\alpha_{\mathrm{fb}}$ in e-liquids accurately and may be of interest to those concerned with addiction and regulation.

In summary, $\alpha_{\mathrm{fb}}$ of e-liquids can be determined directly by ${ }^{1} \mathrm{H}$ NMR using protonation-dependent chemical shifts for nicotine. In a small number of tests, $\alpha_{\mathrm{fb}}$ values were found to be largely unaffected by the vaping process. Of the products tested, only the JUUL liquids were found to combine high nicotine levels with low $\alpha_{\mathrm{fb}}$ values. Pharmacokinetic uptake rates for nicotine may vary among the products, and certainly tobacco company documents (e.g., Chen) ${ }^{13}$ suggest that products with high nicotine levels but low $\alpha_{\mathrm{fb}}$ such as JUUL will yield vape aerosols of much reduced harshness as compared to products with even only moderate nicotine levels but $\alpha_{\mathrm{fb}} \approx 1$. This may well contribute to the current use prevalence ${ }^{21}$ of JUUL products among youth.

\subsection{Author Information}

\subsubsection{Corresponding Author}

*E-mail: peytond@pdx.edu.

\subsubsection{ORCID}

David H. Peyton: 0000-0001-5828-055X 


\subsubsection{Funding}

This work was supported by the U.S. National Institutes of Health, grant R01ES025257. Research reported was supported by the NIEHS and FDA Center for Tobacco Products (CTP). The content is solely the responsibility of the authors and does not necessarily represent the views of the NIH or the FDA.

4.3.4. Notes

The authors declare no competing financial interest.

\subsection{Acknowledgments}

Thank you to Kevin McWhirter for the preparation of some of the sample mixtures.

4.5. Abbreviations

PG, propylene glycol; GL, glycerol; e-cigarettes, electronic cigarettes

\subsection{Contributions}

DHP directed the project, DHP \& JFP conceived the plan, AKD conducted the work, and AKD, JFP, \& DHP wrote the manuscript. 


\subsection{References}

(1) U.S. Census Bureau (2017) QuickFacts, United States; U.S. Census Bureau: Washington, DC, https://www.census.gov/quickfacts/fact/table/US/PST045216 (accessed May 21, 2018).

(2) Centers for Disease Control and Prevention (2018) Smoking and Tobacco Use; Electronic Cigarettes; CDC: Atlanta, GA, http://www.cdc.gov/tobacco/basic_information/e-cigarettes/ (accessed May 21, 2018).

(3) Jamal, A., Gentzke, A., Hu, S. S., Cullen, K. A., Apelberg, B. J., Homa, D. M., and King, B. A. (2017) Tobacco Use Among Middle and High School Students-United States, 2011-2016. Mmwr-Morbid Mortal W 66, 597-603.

(4) Shahab, L., Goniewicz, M. L., Blount, B. C., Brown, J., McNeill, A., Alwis, K. U., Feng, J., Wang, L. Q., and West, R. (2017) Nicotine, Carcinogen, and Toxin Exposure in Long-Term E-Cigarette and Nicotine Replacement Therapy Users A Cross-sectional Study. Ann. Intern. Med. 166, 390-400.

(5) Soneji, S. S., Sung, H. Y., Primack, B. A., Pierce, J. P., and Sargent, J. D. (2018) Quantifying population-level health benefits and harms of e-cigarette use in the United States. PLoS One 13, e0193328.

(6) Kaur, G., Pinkston, R., McLemore, B., Dorsey, W. C., and Batra, S. (2018) Immunological and toxicological risk assessment of ecigarettes. Eur. Respir. Rev. 27, 170119.

(7) Pankow, J. F., Barsanti, K. C., and Peyton, D. H. (2003) Fraction of free-base nicotine in fresh smoke particulate matter from the Eclipse "cigarette" by 1 H NMR spectroscopy. Chem. Res. Toxicol. 16, 23-27.

(8) El-Hellani, A., El-Hage, R., Baalbaki, R., Salman, R., Talih, S., Shihadeh, A., and Saliba, N. A. (2015) Free-Base and Protonated Nicotine in Electronic Cigarette Liquids and Aerosols. Chem. Res. Toxicol. 28, 1532-1537.

(9) Barlow, R. B., and Hamilton, J. T. (1962) Effects of pH on the activity of nicotine and nicotine monomethiodide on the rat diaphragm preparation. Br. J. Pharmacol. Chemother. $18,543-549$.

(10) Pankow, J. F. (2001) A consideration of the role of gas/particle partitioning in the deposition of nicotine and other tobacco smoke compounds in the respiratory tract. Chem. Res. Toxicol. 14, 1465- 1481.

(11) Hurt, R. D., and Robertson, C. R. (1998) Prying open the door to the tobacco industry's secrets about nicotine - The Minnesota Tobacco Trial. Jama-J. Am. Med. Assoc 280, 1173-1181. 
(12) Wayne, G. F., Connolly, G. N., and Henningfield, J. E. (2006) Brand differences of free-base nicotine delivery in cigarette smoke: the view of the tobacco industry documents. Tobacco Control 15, 189- 198.

(13) Chen, L. (1976) pH of Smoke: A Review, Report Number N170, internal document of Lorillard Tobacco Company; Lorillard Tobacco Company: Greensboro, NC; 18 pp, Bates nos. 00118164/ 8181, https://www.industrydocumentslibrary.ucsf.edu/tobacco/docs/ \#id=ykhn0101 (accessed May 21, 2018).

(14) Lephardt, J. (1991) Acid/Base Character of Tobacco Smoke, interoffice correspondence, internal document of Philip Morris, U.S.A.; Philip Morris: Richmond, VA; 5 pp, Bates nos. 2022945340/5344, https://www.industrydocumentslibrary.ucsf.edu/tobacco/docs/\#id= frwp0124 (accessed May 21, 2018).

(15) (1966) Determination of Extractable Nicotine Alkaloids in Smoke Apparatus. In British American Tobacco Records; Bates nos. 100066367/6370, https://www.industrydocumentslibrary.ucsf.edu/ tobacco/docs/jlmd0195 (accessed May 21, 2018).

(16) Pankow, J. F., Tavakoli, A. D., Luo, W. T., and Isabelle, L. M. (2003) Percent free base nicotine in the tobacco smoke particulate matter of selected commercial and reference cigarettes. Chem. Res. Toxicol. 16, 1014-1018.

(17) Barsanti, K. C., Luo, W., Isabelle, L. M., Pankow, J. F., and Peyton, D. H. (2007) Tobacco smoke particulate matter chemistry by NMR. Magn. Reson. Chem. 45, 167-170.

(18) Whidby, J. F., Bassfield, R., and Ferguson, R. N. (1975) An NMR Method for the Determination of Free Nicotine Base of Cigarette Smoke Condensate. In 29th Tobacco Chemist's Research Conference, Beltsville College Park, MD; Bates nos. 10018238381001823849, https://www.industrydocumentslibrary.ucsf.edu/ tobacco/docs/\#id=krvj0037 (accessed May 21, 2018).

(19) Stepanov, I., and Fujioka, N. (2015) Bringing attention to ecigarette $\mathrm{pH}$ as an important element for research and regulation. Tobacco Control 24, 413-414.

(20) Lisko, J. G., Tran, H., Stanfill, S. B., Blount, B. C., and Watson, C. H. (2015) Chemical Composition and Evaluation of Nicotine, Tobacco Alkaloids, $\mathrm{pH}$, and Selected Flavors in E-Cigarette Cartridges and Refill Solutions. Nicotine Tob. Res. 17, 1270-1278.

(21) Willett, J. G., Bennett, M., Hair, E. C., Xiao, H., Greenberg, M. S., Harvey, E., Cantrell, J., and Vallone, D. (2018) Recognition, use and perceptions of JUUL among youth and young adults. Tob Control, 054273. 
4.8. Supporting Information: Free-base Nicotine Determination in Electronic Cigarette Liquids by ${ }^{1} \mathrm{H}$ NMR Spectroscopy

\subsubsection{Materials and Methods}

Glacial acetic acid was obtained from Mallinckrodt Chemicals (Staines-uponThames, England). Tertbutylamine (98\%), USP grade propylene glycol, and USP grade glycerol were obtained from SigmaAldrich (St. Louis, MO). (S)-(-)-nicotine, 99\%, was obtained from Alfa Aesar (Haverhill, MA). DMSO-d6, D 99.9\%, was obtained from Cambridge Isotope Laboratories, Inc. (Andover, MA). Precision coaxial NMR inserts (WGS-5BL-SP and WGS-5BL) and precision NMR tubes (535-PP-7) were purchased from Wilmad (Vineland, NJ).

Commercial e-liquids tested included: 'Maui' by Nicquid (6 and $24 \mathrm{mg} / \mathrm{mL} \mathrm{Nic}$; Miamisburg, OH), 'Snake Oil' by Seduce Juice (12 mg/mL Nic; Charlotte, NC), 'No. 88' by Beard Vape Co. (6 mg/mL Nic; Los Angeles, CA), 'Snow White's Demise' by The Mad Alchemist (12 mg/mL Nic; London, KY), 'Zen' by the Mad Alchemist (18 mg/mL Nic), 'Taurus' by Twelve Vapor (3 mg/mL Nic; Buffalo, NY), 'Galactica' by Space Jam (3 mg/mL Nic; San Clemente, CA), 'Crème Brulee' by JUUL (59 mg/mL Nic; San Francisco, CA), 'Fruit Medley' by JUUL (59 mg/mL Nic), and 'Placid' by Adirondack Vapor (3 mg/mL Nic; New Hartford, NY).

Preparation of Free-base and Monoprotonated Standards: Propylene glycol (PG) and glycerol (GL) were combined in a molar ratio of 54:46 (as determined by NMR), with a nicotine concentration of $24 \mathrm{mg} / \mathrm{mL}$. Aliquots were independently combined with acid (acetic acid, 5:1 nicotine, by mol) and base (tert-butylamine, 1:1 nicotine, by mol) to 
produce monoprotonated and free-base (unprotonated) nicotine. Other acids tested included chloroacetic acid, dichloroacetic acid, trichloroacetic acid, and hydrochloric acid, but these caused diprotonation of the nicotine, and so were not used for the preparation of monoprotonated nicotine standard solutions in PG/GL mixtures.

NMR Spectroscopy: E-liquid samples were placed in a precision coaxial NMR insert. The lock solvent, DMSO-d6, was placed in an outer precision NMR tube. ${ }^{1} \mathrm{H}$ NMR experiments were conducted using a TXI probe and at $40{ }^{\circ} \mathrm{C}$ in order to increase the molecular tumbling rate, therefore improving the shim. A zg30 pulse was applied, 16 scans were collected, a relaxation delay (D1) of 3 seconds was used, the size of the real spectrum (TD) was 65536, the spectral width (SW) was 15 ppm with the transmitter frequency offset (O1P) set to $6 \mathrm{ppm}$, and the total experiment time was 2 minutes.

Sample Collection from Vaping: Samples containing low and high quantities of $\alpha_{\mathrm{fb}}$, respectively, were vaporized using the CORESTA sample collection protocol 1 directly into a concentric inner NMR tube sealed with a rubber stopper (Wilmad). 'Zen' flavor or 'Maui' flavor (24 mg/mL Nic) e-liquid was vaporized using a Kangertech Subtank Mini (1.2 $\Omega$ coil) plus a KBOX Mini (Kangertech; Shenzen, China) at 22 watts for 'Zen' or 20 watts for 'Maui'. E-liquid from a 'Crème Brulee' JUUL pod was vaporized using a JUUL device with no customizable settings. Each device was connected to an NMR tube using a short piece of silicone tubing (Cole-Parmer; Vernon Hills, IL) with a $1.2 \times 25 \mathrm{~mm}$ 18-gauge needle (BD; Franklin Lakes, NJ) at the end to enter the rubber septum on top of the NMR tube. A second needle was placed through the rubber septum to allow air to flow through the system. This second needle was then 
attached to a second short piece of tubing, which was connected to an NE1660 syringe pump (New Era Pump Systems, Inc.; Farmingdale, NY).

\subsection{2. $\quad \mathrm{pH}$ of Water Dilutions of Electronic Cigarette Fluids}

Neglecting the diprotonated species $\mathrm{NicH}_{2}{ }^{2+}$, the fraction of nicotine in the free-base form is

$$
\alpha_{\mathrm{fb}} \equiv \frac{[\mathrm{Nic}]}{[\mathrm{Nic}]+\left[\mathrm{NicH}^{+}\right]}
$$

and the total nicotine is given by

$$
\mathrm{Nic}_{\mathrm{T}}=[\mathrm{Nic}]+\left[\mathrm{NicH}^{+}\right]
$$

(All concentrations have units of molarity.) For a dilute water solution, activity corrections can be neglected so that solution activities equal solution concentrations. Then,

$$
K_{\mathrm{a}} \equiv \frac{[\mathrm{Nic}]\left[\mathrm{H}^{+}\right]}{\left[\mathrm{NicH}^{+}\right]}
$$

where $K_{\mathrm{a}}$ is the second dissociation constant for $\mathrm{NicH}_{2}{ }^{2+}$. At $25{ }^{\circ} \mathrm{C}$, a reported value for $K_{\mathrm{a}}$ in water is $10^{-8.012}$. By (S-1) and (S-3),

$$
\alpha_{\mathrm{fb}}^{\text {water }}=\frac{1}{1+10^{-\mathrm{pH} / K_{\mathrm{a}}}}
$$

Attempts have been made to determine $\alpha_{\mathrm{fb}}$ for e-liquids by diluting an e-liquid with water, measuring the $\mathrm{pH}$, then calculating $\alpha_{\mathrm{fb}}^{\text {water }}$ by (S-4). Such values may be quite different from the $\alpha_{\mathrm{fb}}$ values in the original e-liquids because the solution matrix has been 
drastically changed, the water dilutes the original sample, and because the solubility of air-related $\mathrm{CO}_{2}$ can introduce carbonic acid beyond that already present in the e-liquid.

It is likely that the effect of $\mathrm{CO}_{2}$ incursion is visible in the data of ${ }^{3}$, who dissolved $0.5 \mathrm{~g}(\sim 0.5 \mathrm{~mL})$ of each of various e-liquids in $5 \mathrm{~mL}$ "distilled deionized" water giving a 1:11 dilution. The $\mathrm{pH}$ was measured over 1 hour. Taking $\mathrm{p} K_{\mathrm{a}}=8.01$ for $\mathrm{NicH}^{+}$in water $25^{\circ} \mathrm{C}^{2}$, then if no other acid/base-active constituents are in a given e-liquid, the expected $\mathrm{pH}$ values for the nicotine dilutions can be calculated for two cases: 1) no- $\mathrm{CO}_{2}$; and 2) with equilibrium with atmospheric $\mathrm{CO}_{2}$ at $10^{-3.40}$ atm.

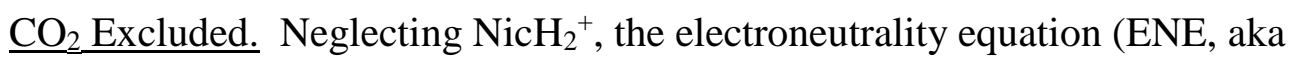
“charge balance equation") is

$$
[\mathrm{H}+]+[\mathrm{NicH}+]=[\mathrm{OH}-] \quad(\mathrm{CO} 2 \text { excluded })
$$

Nicotine is a base, so the lowest $\mathrm{pH}$ that can result at $25^{\circ} \mathrm{C}$ for $(\mathrm{S}-5)$ is $\mathrm{pH}=7.00\left(\left[\mathrm{H}^{+}\right]=\right.$ $\left[\mathrm{OH}^{-}\right]$, no nicotine in the solution).

$$
\left[\mathrm{NicH}^{+}\right]=\left(1-\alpha_{\mathrm{fb}}^{\text {water }}\right) \mathrm{Nic}
$$

For dilute water conditions so that activity corrections can be neglected and if the aqueous sample is behaving like a $\sim 100 \%$ water solution, making substitutions in (S-6) gives

$$
\left[\mathrm{H}^{+}\right]+\left(1-\alpha_{\mathrm{fb}}^{\text {water }}\right) \mathrm{Nic}_{\mathrm{T}}=K_{\mathrm{W}} /\left[\mathrm{H}^{+}\right]\left(\mathrm{CO}_{2} \text { excluded }\right)
$$

Where $K_{\mathrm{w}}$ is the ionization dissociation constant for water; at $25^{\circ} \mathrm{C}$ with $K_{\mathrm{w}}=1.01 \mathrm{X} 10^{-}$

${ }^{14}$. Since $\alpha_{\mathrm{fb}}^{\text {water }}$ is a function of $\left[\mathrm{H}^{+}\right]$, once $\mathrm{Nic}_{\mathrm{T}}$ is specified, only one positive real value 120 
of $\left[\mathrm{H}^{+}\right]$satisfies $(\mathrm{S}-7)$. Table $\mathrm{S}-1$ gives the computed $\mathrm{pH}$ values for the dilutions of ${ }^{3}$, and their measured values. The computed values are plotted in Figure S-1 (dashed line). The agreement is not good; all of the measured $\mathrm{pH}$ values are significantly lower than the values obtained with (S-7). The average [measured - computed] deviation (signed) is 1.32, which strongly suggests the presence of some acid(s) in most/all of the samples.

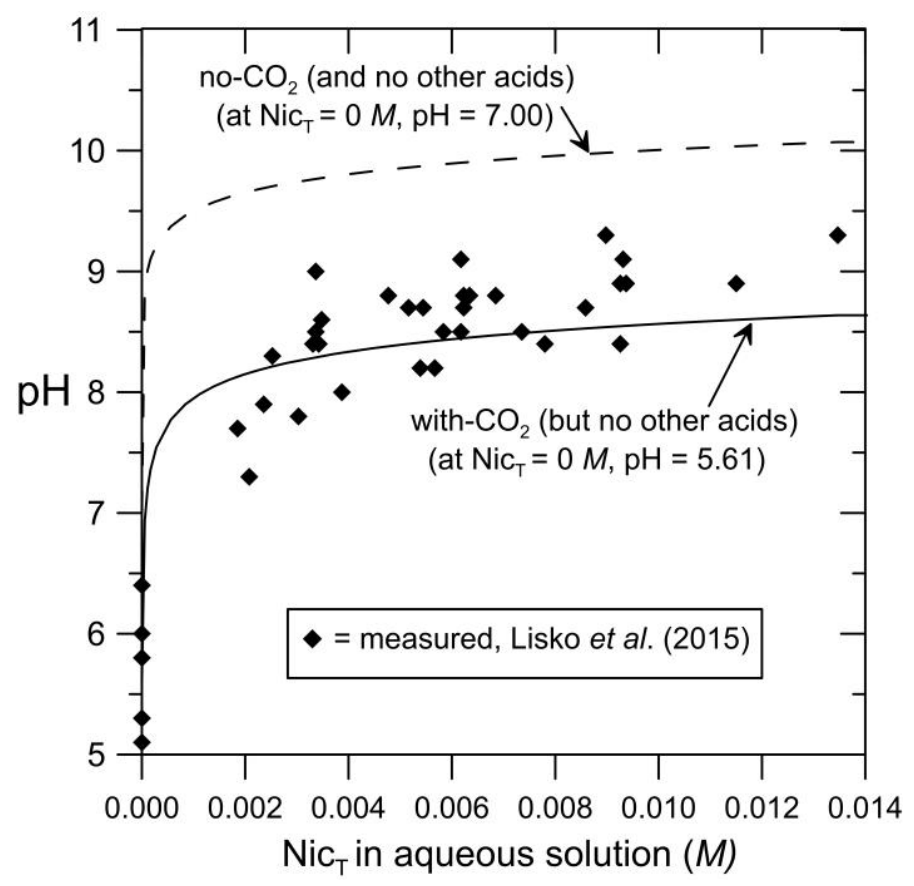

Figure S-1. Measured pH values in water dilutions of e-liquids by Lisko et al. (2015), compared to predicted values for the post-dilution nicotine concentrations with and without consideration of equilibrium with atmospheric $\mathrm{CO}_{2}$. 
Table S-1. Measured pH values in water dilutions of e-liquids by Lisko et al. (2015), and calculated values for the post-dilution nicotine concentrations with and without consideration of equilibrium with atmospheric $\mathrm{CO}_{2}$.

\begin{tabular}{|c|c|c|c|c|c|}
\hline \multirow[b]{2}{*}{$\underline{\text { South Beach Smoke }}$} & \multirow{2}{*}{$\begin{array}{l}\text { nicotine, } \\
\text { e-liquid } \\
\text { mg/g }\end{array}$} & \multirow{2}{*}{$\begin{array}{c}\text { nicotine, } \\
\text { water dilution } \\
\mathrm{m} M\end{array}$} & \multicolumn{3}{|c|}{$\mathrm{pH}$} \\
\hline & & & measured & $\begin{array}{l}\text { no } \mathrm{CO}_{2} \\
\text { calculated }\end{array}$ & $\begin{array}{l}\text { with } \mathrm{CO}_{2}, \\
\text { calculated }\end{array}$ \\
\hline Vanilla & 0 & 0 & 5.3 & 7.00 & 5.61 \\
\hline Tobacco Blue & 4.2 & 2.36 & 7.9 & 9.68 & 8.19 \\
\hline Tobacco & 4.5 & 2.53 & 8.3 & 9.70 & 8.21 \\
\hline Peppermint & 9.2 & 5.16 & 8.7 & 9.86 & 8.40 \\
\hline Tobacco Gold & 9.7 & 5.44 & 8.7 & 9.87 & 8.41 \\
\hline Peach & 12.2 & 6.85 & 8.8 & 9.92 & 8.47 \\
\hline Menthol & 13.1 & 7.35 & 8.5 & 9.93 & 8.49 \\
\hline \multicolumn{6}{|l|}{$\underline{V 2}$} \\
\hline Menthol & 0 & 0 & 6.4 & 7.00 & 5.61 \\
\hline Sahara & 5.4 & 3.03 & 7.8 & 9.74 & 8.26 \\
\hline Red & 5.9 & 3.31 & 8.4 & 9.76 & 8.28 \\
\hline Pepper & 9.6 & 5.39 & 8.2 & 9.87 & 8.41 \\
\hline Sahara & 11.0 & 6.17 & 8.5 & 9.90 & 8.44 \\
\hline Menthol & 15.3 & 8.59 & 8.7 & 9.97 & 8.52 \\
\hline Red & 16.7 & 9.37 & 8.9 & 9.99 & 8.54 \\
\hline \multicolumn{6}{|l|}{$\underline{\text { Premium }}$} \\
\hline Cherry & 0 & 0 & 5.3 & 7.00 & 5.61 \\
\hline Coffee & 0 & 0 & 5.8 & 7.00 & 5.61 \\
\hline Watermelon & 3.3 & 1.85 & 7.7 & 9.63 & 8.12 \\
\hline Blueberry & 3.7 & 2.08 & 7.3 & 9.66 & 8.16 \\
\hline Pineapple & 6.9 & 3.87 & 8.0 & 9.79 & 8.32 \\
\hline Menthol & 8.5 & 4.77 & 8.8 & 9.84 & 8.38 \\
\hline Pear & 10.1 & 5.67 & 8.2 & 9.88 & 8.42 \\
\hline Vanilla & 13.9 & 7.80 & 8.4 & 9.95 & 8.50 \\
\hline Peach & 16.5 & 9.26 & 8.4 & 9.98 & 8.54 \\
\hline
\end{tabular}




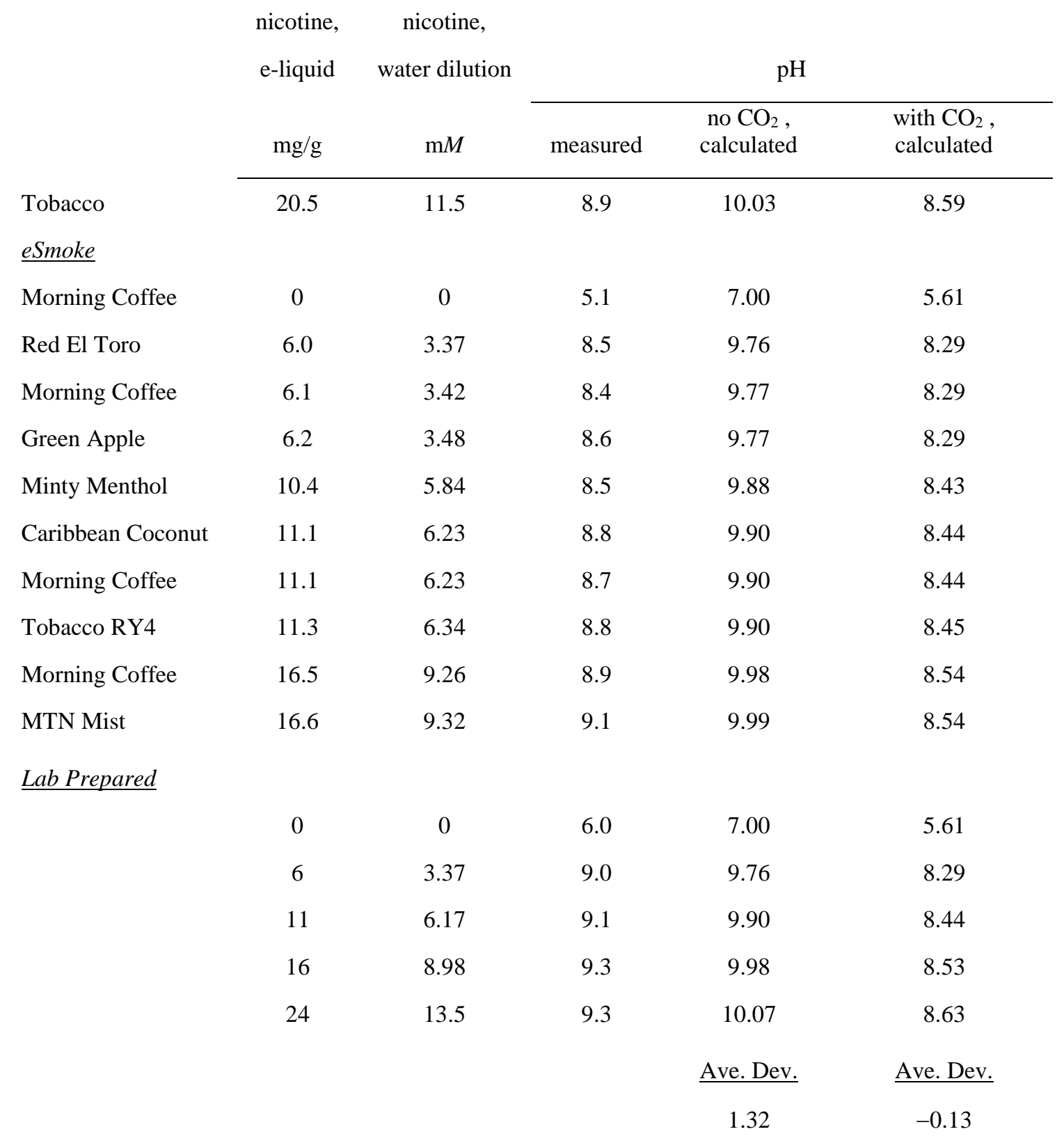




\subsubsection{Supporting Information References}

(1) CORESTA. (2018) Routine Analytical Machine for E-Cigarette Aerosol Generation and Collection - Definitions and Standard Conditions, https://www.coresta.org/routine-analyticalmachine-e-cigarette-aerosol-generationand-collection-definitions-and-standard.

(2) Barlow, R. B., and Hamilton, J. T. (1962) Effects of $\mathrm{pH}$ on the activity of nicotine and nicotine monomethiodide on the rat diaphragm preparation. Br J Pharmacol Chemother 18, 543-549.

(3) Lisko, J. G., Tran, H., Stanfill, S. B., Blount, B. C., and Watson, C. H. (2015) Chemical Composition and Evaluation of Nicotine, Tobacco Alkaloids, $\mathrm{pH}$, and Selected Flavors in E-Cigarette Cartridges and Refill Solutions. Nicotine Tob Res $17,1270-1278$ 
5. Nicotine in Tobacco Product Aerosols: "It's Déjà Vu All Over Again"

Anna K. Duell, ${ }^{\dagger}$ James F. Pankow ${ }^{\dagger \dagger} \&$ \&avid H. Peyton ${ }^{\dagger}$

${ }^{\dagger}$ Department of Chemistry, Portland State University, Portland, Oregon 97207-0751, USA

₹ Department of Civil and Environmental Engineering, Portland State University, Portland, Oregon 97207-0751, USA

This article has been accepted for publication in:

Duell, Anna K., James F. Pankow, and David H. Peyton. "Nicotine in Tobacco Product Aerosols: "It's Déjà Vu All Over Again" Tobacco Control. Epub ahead of print: 2019.

following peer review and can also be accessed online at http://dx.doi.org/10.1136/tobaccocontrol-2019-055275.

Keywords: nicotine; free-base nicotine; nicotine salts; nic salts, JUUL; electronic cigarettes; e-cigarettes; ENDS; pods; pod systems; vaping. 


\subsection{Abstract}

Introduction The distribution of nicotine among its free-base ( $\mathrm{fb}$ ) and protonated forms in aerosolized nicotine affects inhalability. It has been manipulated in tobacco smoke and now in electronic cigarettes by use of acids to de-freebase nicotine and form 'nicotine salts'.

Methods Measurements on electronic cigarette fluids (e-liquids) were carried out to determine (1) fraction of nicotine in the free-base form $\left(\alpha_{\mathrm{fb}}\right)$ and (2) the levels of organic acid(s) and nicotine. Samples included JUUL 'pods', 'look-a-like/knockoff' pods, and some bottled 'nicotine salt' and 'non-salt' e-liquids.

Results $\alpha_{\mathrm{fb}}=0.12 \pm 0.01$ at $40{ }^{\circ} \mathrm{C}\left(\approx 37^{\circ} \mathrm{C}\right)$ for $10 \mathrm{JUUL}$ products, which contain benzoic acid; nicotine protonation is extensive but incomplete.

Discussion First-generation e-liquids have $\alpha_{\mathrm{fb}} \approx 1$. At cigarette-like total nicotine concertation ( $\mathrm{Nic}_{\text {tot }}$ ) values $\sim 60 \mathrm{mg} / \mathrm{mL}$, e-liquid aerosol droplets with $\alpha_{\mathrm{fb}} \approx 1$ are harsh upon inhalation. The design evolution for e-liquids has paralleled that for tobacco products giving a 'déjà vu' trajectory for $\alpha_{\mathrm{fb}}$. For $17^{\text {th }}$-century 'air-cured' tobacco, $\alpha_{\mathrm{fb}}$ in the smoke particles was likely $\geq 0.5$. The product $\alpha_{\mathrm{fb}} \mathrm{Nic}_{\mathrm{tot}}$ in the smoke particles was high. 'Flue-curing' retains higher levels of leaf organic acids, resulting in $\alpha_{\mathrm{fb}} \approx 0.02$ and lowered harshness. Some tobacco cigarette formulations/designs have been adjusted to restore some nicotine sensory "kick"/impact with $\alpha_{\mathrm{fb}} \approx 0.1$, as for Marlboro. Overall, for tobacco smoke the de-freebasing trajectory was $\alpha_{\mathrm{fb}} \geq 0.5 \rightarrow \sim 0 \rightarrow \sim 0.1$, as compared to $\alpha_{\mathrm{fb}}=\sim 1 \rightarrow \sim 0.1$ for e-cigarettes. For JUUL the result has been, perhaps, an optimized, flavoured nicotine delivery system. The design evolution for e-cigarettes has made them 
more effective as substitutes to get smokers off combustibles. However, this evolution has likely made e-cigarette products vastly more addictive for never-smokers. 


\subsection{Introduction}

Nicotine can exist in a free-base (fb) form and in two protonated forms (Figure 1). For electronic cigarette fluids (e-liquids) and the aerosolized droplets created therefrom, both the total nicotine concentration $\left(\mathrm{Nic}_{\text {tot }}\right)$ and the fraction of nicotine in the free-base form $\left(\alpha_{\mathrm{fb}}\right)$ can vary. ${ }^{1} \mathrm{Fb}$ nicotine is volatile and gaseous fb nicotine is directly sensable. Protonated nicotine is not volatile and so has been referred to in the tobacco industry as 'bound nicotine.'2 First generation e-liquids were simply fb nicotine dissolved in a mix of propylene glycol (PG) and/or glycerol (GL), with $\alpha_{\mathrm{fb}}=1$, and Nic tot in the range of 6$24 \mathrm{mg} / \mathrm{mL}$. In comparison, in the droplets making up tobacco smoke particulate matter (PM), Nic tot values are typically much higher $(\sim 60 \mathrm{mg} / \mathrm{mL}) .{ }^{3}$ Electronic cigarette (eciagrette) aerosols with high values of the product $\alpha_{\mathrm{fb}} \mathrm{Nic}_{\text {tot }}$ can be expected to be harsh upon inhalation, as with $\alpha_{\mathrm{fb}}=1$ and Nic $\mathrm{tot}=60 \mathrm{mg} / \mathrm{mL} .{ }^{1}$ Non-harsh cigarette-like nicotine levels in aerosolized e-liquids therefore require $\alpha_{\mathrm{fb}}<<1$. This can be achieved by the addition of an acid to the PG/GL/nicotine mix, for example benzoic acid, as in the JUUL product line.

Given the large market share quickly achieved by $\mathrm{JUUL}^{45}$ and its youth-oriented e-cigarette demographic, ${ }^{6}$ the goal of this work was to determine $\alpha_{\mathrm{fb}}$ values and acid levels in the e-liquids from JUUL and look-a-like/knock-off product $^{7}$ competitors, available as of October 2018, and thereby characterize the use of acid additives to moderate fb nicotine delivery, and thus harshness, while maintaining high total nicotine delivery. The measured $\alpha_{\mathrm{fb}}$ values were compared with those for first generation ecigarette products. The first-generation e-cigarette $\rightarrow$ JUUL trajectory is compared with 
that for the smoke aerosol from colonial-era tobacco $\rightarrow$ flue-cured tobacco $(1850 \mathrm{~s}$

forward) $\rightarrow$ the modern Marlboro cigarette. The measurements were carried out by application of ${ }^{1} \mathrm{H}$ NMR spectroscopy (hereafter, NMR). ${ }^{189}$ As outlined by Duell et al. (2018), ${ }^{1} \mathrm{NMR}$ is a method which allows the reliable determination of $\alpha_{\mathrm{fb}}$ values in eliquids without any alteration of the sample, for example, without water addition, which changes nicotine protonation chemistry. The e-liquid results are examined in the context of the acid+nicotine first protonation equilibrium constant.

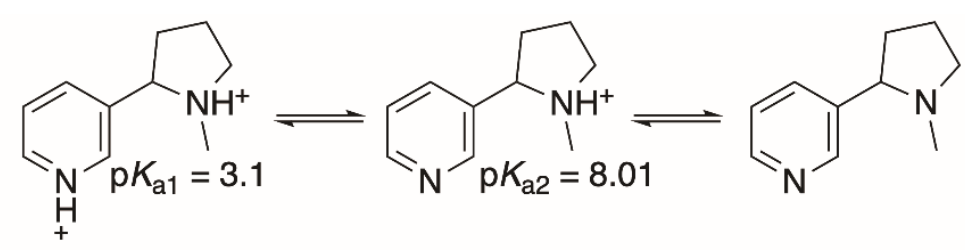

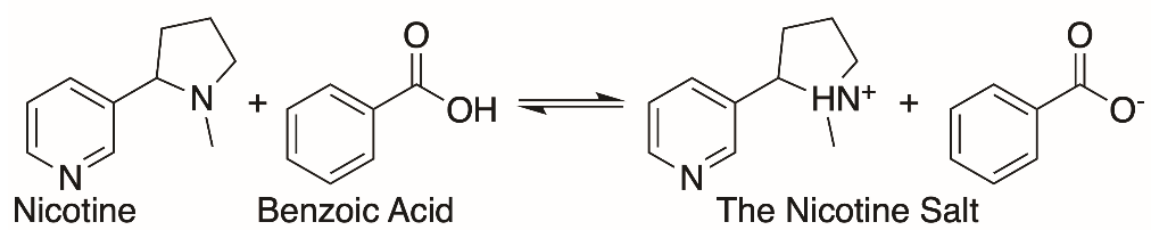

Figure 1. Top: the distribution of nicotine in vape and tobacco aerosols primarily involves two forms; centre: $\mathrm{NicH}^{+}$(monoprotonated) which is non-volatile; and right: free-base $(\mathrm{fb})$ nicotine, which is volatile. The fraction of $\mathrm{fb}\left(\alpha_{\mathrm{fb}}\right)$ depends on the $\mathrm{acid} / \mathrm{base}$ conditions. In water at $25^{\circ} \mathrm{C}, \mathrm{p} K_{\mathrm{a}, 2}=8.01$. Bottom: so-called 'nicotine salts' in electronic cigarette liquids are formed by adding an organic acid (benzoic acid is depicted here) to the formulation, producing a lower $\alpha_{\mathrm{fb}}$ that depends on the ratio of nicotine:acid, as well as temperature and solvent conditions. 


\subsubsection{Nicotine Protonation and $\alpha_{\mathrm{fb}}$}

Predicting the extent of nicotine protonation (including the fraction $\alpha_{\mathrm{fb}}{ }^{10}$ ) in any solution requires knowledge of the governing acid/base concentrations and their mediumdependent equilibrium constants. Fully protonated nicotine carries two protons (figure 1)

with acidity constants $K_{\mathrm{a}}^{\mathrm{NicH}_{2}^{2+}}\left(=K_{\mathrm{a}, 1}\right)$ and $K_{\mathrm{a}}^{\mathrm{NicH}^{+}}\left(=K_{\mathrm{a}, 2}\right)$. Measurement of $K_{\mathrm{a}}$ values in tobacco smoke and e-liquids is very difficult but relatively easy in water. In water,

reported values at $25^{\circ} \mathrm{C}$ are $\mathrm{p} K_{\mathrm{a}}^{\mathrm{NicH}^{+}}=8.01$, and $\mathrm{p} K_{\mathrm{a}}^{\mathrm{NicH}_{2}^{2+}}=3.10 .{ }^{11}$ At $37^{\circ} \mathrm{C}$ the values are 7.65 and 2.77 , respectively. ${ }^{12}$ In water, $\mathrm{pH} \leq 4$ is required for significant $(\geq 10 \%)$ $\mathrm{NicH}_{2}^{2+}$.

When conditions are such that there is no equivalent excess of acid over nicotine (so that the total molar-based concentration of monoprotic acid $\left(\mathrm{C}_{\mathrm{HA}}\right) /$ total molar-based concentration of nicotine $\left(\mathrm{C}_{\mathrm{Nic}}\right)$ is $\left.\leq 1\right)$, or the protonating acid is weak for the medium, $\mathrm{NicH}_{2}^{2+}$ can be neglected and the dominant protonation of $\mathrm{fb}$ nicotine (Nic) occurs according to

$$
\mathrm{Nic}+\mathrm{H}^{+}=\mathrm{NicH}^{+} \quad \frac{\left[\mathrm{NicH}^{+}\right]}{[\mathrm{Nic}]\left[\mathrm{H}^{+}\right]} \equiv\left(K_{\mathrm{a}}^{\mathrm{NicH}^{+}}\right)^{-1}
$$

so that

$$
\alpha_{\mathrm{fb}} \equiv \frac{[\mathrm{Nic}]}{[\mathrm{Nic}]+\left[\mathrm{NicH}^{+}\right]+\left[\mathrm{NicH}_{2}^{2+}\right]} \approx \frac{[\mathrm{Nic}]}{[\mathrm{Nic}]+\left[\mathrm{NicH}^{+}\right]}
$$

The diprotonated form may not be negligible for all e-liquids, including some non-JUUL high-acid brands examined experimentally here. Each bracketed term in equations (1) and (2) is a molar concentration (and not a chemical activity) so that $K_{\mathrm{a}}^{\mathrm{NicH}^{+}}$and all the other 
$K$ values herein are constant medium-type equilibrium constants, analogous to ${ }^{\mathrm{c}} K$ values as discussed by Pankow et al, ${ }^{13}$ and dependent on the nature of the particular solution medium.

\subsubsection{Net Protonation Reaction}

In a liquid medium (eg, the PG/GL matrix and water) the acid dissociation reaction of an acid, HA (benzoic acid, acetic acid), is

$$
\mathrm{HA}=\mathrm{H}^{+}+\mathrm{A}^{-} \quad \frac{\left[\mathrm{H}^{+}\right]\left[\mathrm{A}^{-}\right]}{[\mathrm{HA}]} \equiv K_{\mathrm{a}}^{\mathrm{HA}}
$$

The overall reaction for monoprotonation of Nic by HA is given by equations (1) and (3), so that

$$
\mathrm{HA}+\mathrm{Nic}=\mathrm{A}^{-}+\mathrm{NicH}^{+} \quad \frac{\left[\mathrm{A}^{-}\right]\left[\mathrm{NicH}^{+}\right]}{[\mathrm{HA}][\mathrm{Nic}]}=\frac{K_{\mathrm{a}}^{\mathrm{HA}}}{K_{\mathrm{a}}^{\mathrm{NiCH}^{+}}} \equiv K_{\mathrm{oa}, 1}
$$

$K_{\mathrm{oa}, 1}$ is dimensionless because both the forward and backward reactions are bimolecular: any mol-proportional concentration scale can be used. For water, $K_{\mathrm{oa}, 1}$ values for different acids can be calculated; $K_{\mathrm{a}}^{\mathrm{NicH}^{+}}$values and $K_{\mathrm{a}}^{\mathrm{HA}}$ values for many important acids are individually well known because $\mathrm{pH}$ is easily measured in water: at $37^{\circ} \mathrm{C}$, for benzoic acid and vanillin (a common e-liquid flavour additive), $\mathrm{p} K_{\mathrm{a}}^{\mathrm{HA}}=4.20$ and 7.27, respectively. ${ }^{14}$ For these two acids with nicotine in water at $37^{\circ} \mathrm{C}$, then $K_{\mathrm{oa}, 1}=10^{3.45}$ and $10^{0.38}$, respectively. In contrast, in PG and GL, either individually or as a mixture, $K_{\mathrm{a}}^{\mathrm{NicH}^{+}}$and $K_{\mathrm{a}}^{\mathrm{HA}}$ values for relevant acids are unknown. The species $\mathrm{H}^{+}$, however, does not appear in equation (4), and so values of $K_{\mathrm{oa}, 1}$ values can be directly measured in PG and GL solutions/mixtures. 
Let $C_{\mathrm{HA}}$ and $C_{\mathrm{Nic}}$ be the total molar-based concentrations of HA and nicotine as initially added to a PG/GL solution. $\left(C_{\mathrm{Nic}}\right.$ and $\mathrm{Nic}_{\text {tot }}$ are proportional; $\mathrm{Nic}_{\text {tot }}$ has units of $\mathrm{mg} / \mathrm{mL}$ ). Neglecting formation of the diprotonated species, establishment of a reaction equilibrium will lead to protonation such that $\left[\mathrm{NicH}^{+}\right]=\left[\mathrm{A}^{-}\right]=x$ :

$$
K_{\mathrm{oa}, 1}=\frac{\mathrm{x}^{2}}{\left(C_{\mathrm{HA}}-\mathrm{x}\right)\left(C_{\mathrm{Nic}}-\mathrm{x}\right)}
$$

so that $\alpha_{\mathrm{fb}}=\left(C_{\mathrm{Nic}}-\mathrm{x}\right) / C_{\mathrm{Nic}}$. Because the reaction is bimolecular and $K_{\mathrm{oa}, 1}$ is dimensionless, for any mass concentration of total nicotine, we can set $C_{\mathrm{Nic}}=1$, and $C_{\mathrm{HA}}$ $=C_{\mathrm{HA}} / C_{\mathrm{Nic}}$. Then, $\alpha_{\mathrm{fb}}=1-x$, and

$$
K_{o a, 1}=\frac{\left(1-\alpha_{\mathrm{fb}}\right)^{2}}{\left(\frac{C_{\mathrm{HA}}}{C_{\mathrm{Nic}}}-1+\alpha_{\mathrm{fb}}\right) \alpha_{\mathrm{fb}}}
$$

When $K_{\mathrm{oa}, 1}$ and $C_{\mathrm{HA}} / C_{\mathrm{Nic}}$ are known, then equation (6) can be solved for $\alpha_{\mathrm{fb}}$ either numerically or by the quadratic equation. For the latter, $a=K_{\mathrm{oa}, 1}-1, b=\left(K_{\mathrm{oa}, 1} C_{H A} / C_{N i c}\right.$ $\left.-K_{\mathrm{oa}, 1}+2\right)$, and $c=-1$; the root $\alpha_{\mathrm{fb}}=\left(-b+\sqrt{b^{2}-4 a c}\right) /(2 a)$ is chosen so that $\alpha_{\mathrm{fb}}>0$. Cases involving $K_{\mathrm{oa}, 1}=1$ are not second order $(a=0)$, and so reduce to $\alpha_{\mathrm{fb}}=1 /(1+$ $\left.C_{\mathrm{HA}} / C_{\mathrm{Nic}}\right)$. When $K_{\mathrm{Oa}, 1}$ is large, the reliability of equations (5-6) will decrease for $C_{\mathrm{HA}} / C_{\mathrm{Nic}}>1$ due to an increasing importance of $\mathrm{NicH}_{2}^{2+}$. For the special case of $C_{\mathrm{HA}} / \mathrm{C}_{\mathrm{Nic}}=1$, then

$$
K_{\mathrm{oa}, 1}=\frac{\left(1-\alpha_{\mathrm{fb}}\right)^{2}}{\alpha_{\mathrm{fb}}{ }^{2}}
$$

and 


$$
\alpha_{\mathrm{fb}}=\frac{1}{1+\sqrt{K_{\text {overall }}}}
$$

\subsection{Laboratory Methods}

\subsubsection{NMR Determinations of $\alpha_{\mathrm{fb}}$, Nicotine and Acid Concentrations}

JUUL e-liquid 'pods' were purchased from JUUL. Other pod brands (ZOOR, SMPO, Myle, ZiiP, and Eon Smoke) and bottles of e-liquids (Fuzion Vapor) were purchased from online suppliers. Bottles of 'nicotine salt' e-liquids (Salt Bae ${ }^{50}$ and Pacha Mama Salts) were purchased from a vape shop in Portland, OR. Glacial acetic acid was obtained from Mallinckrodt Chemicals (Staines-upon-Thames, England). Tertbutylamine (98\%), was obtained from Sigma Aldrich (St. Louis, MO). DMSO- $d_{6}$, D 99.9\%, was obtained from Cambridge Isotope Laboratories, Inc. (Andover, MA). Precision coaxial NMR inserts (WGS-5BL-SP and WGS-5BL) and precision NMR tubes (535-PP-7) were purchased from Wilmad (Vineland, NJ).

Monoprotonated and $\mathrm{fb}$ nicotine standards, which were used to calculate the $\mathrm{fb}$ nicotine fraction in each sample, were prepared by adding acetic acid or tertbutylamine to various e-liquids until the limiting NMR chemical shifts were achieved. In the present study, standards were prepared using the following commercial e-liquids: 'Mango' flavoured JUUL, ‘Apple' flavoured ZOOR, 'Cake' flavoured ZOOR, and 'Blue Raspberry Lemonade' flavoured Salt $\mathrm{Ba}^{50}$. In our previous work, standards were prepared from nicotine-containing PG/GL samples rather than actual commercial eliquids, resulting in small differences in the $\alpha_{\mathrm{fb}}$ values reported here. Various commercial e-liquid standards were prepared because dissimilarities in the e-liquid compositions 
(such as the presence of benzoic acid or levulinic acid) can result in different limiting chemical shifts for the monoprotonated and $\mathrm{fb}$ nicotine reference samples. Appropriate reference samples were matched to the tested commercial e-liquids by using the most similar compositions as determined by analysis of ${ }^{1} \mathrm{H}$ NMR spectra. In particular, this was executed by matching samples and reference standards containing the same primary acids (if present), that is, benzoic acid or levulinic acid. Details for the references used for each sample can be found in online supplementary table S-1. $\alpha_{\mathrm{fb}}$ was calculated using the difference between the chemical shifts of two aromatic nicotine protons and the nicotine methyl resonance, respectively. The average was then calculated ( \pm the difference between the two values divided by two). ${ }^{1}$

Concentric tube samples containing each e-liquid were prepared for $\alpha_{\mathrm{fb}}$ analyses per previous methods, ${ }^{1}$ and samples containing a single drop of each e-liquid in $500 \mu \mathrm{L}$ of DMSO- $d_{6}$ were used for composition analysis, owing to the better shim that can be achieved with a lower sample concentration. A $600 \mathrm{MHz}$ NMR spectrometer was used to execute zg30 ${ }^{1} \mathrm{H}$ experiments utilizing parameters reported previously and HSQC experiments, as needed..$^{1}$ Thus, each e-liquid sample was placed in a precision coaxial NMR insert and the lock solvent, DMSO- $d_{6}$, was placed in the outer $5 \mathrm{~mm}$ NMR tube. ${ }^{1} \mathrm{H}$ NMR experiments were conducted using a TXI probe and at $40^{\circ} \mathrm{C}$ in order to increase the molecular tumbling rate, improving the shim. Sixteen scans were collected using the zg30 pulse sequence; a relaxation delay (D1) of $3 \mathrm{~s}$ between each scan was used; the size of the real spectrum (TD) was 65536 data points; and the spectral width (SW) was 15 ppm with the transmitter frequency offset $(\mathrm{O} 1 \mathrm{P})$ set to $6 \mathrm{ppm}$, giving a total experiment time of 2 min per sample. 
Spectra for composition determination were assessed using integration analysis. After phasing and baseline correction, the chemical components (eg, PG, GL, nicotine, and benzoate or levulinate) were analyzed using the resonance(s) with the least overlap. The resulting integrations were used to calculate the mole per cent of each component, which was then used to calculate the weight per cent (wt \%). Other details about the calculation of free-base nicotine fraction have been reported previously, ${ }^{1}$ except with a modification to the $\mathrm{fb}$ and monoprotonated nicotine standards used as described previously.

\subsection{2. $\quad K_{\mathrm{oa}, 1}$ Determinations}

Based on equation (6), values of $K_{\mathrm{oa}, 1}$ were determined for benzoic acid at $40^{\circ} \mathrm{C}$ in $43 / 57$ PG/GL by weight (48/52 by mol). The mixture was amended with benzoic acid and nicotine to give $C_{\mathrm{HA}}=3.31 \times 10^{-4} \mathrm{~mol} / \mathrm{mL}$ and $C_{\mathrm{Nic}}=3.28 \times 10^{-4} \mathrm{~mol} / \mathrm{mL}\left(C_{\mathrm{HA}} / C_{\mathrm{Nic}}\right.$ $=1.01$, nicotine at $4.6 \mathrm{wt} \%)$. A second mixture was prepared with a PG/GL ratio of 32/68 by weight ( $36 / 64$ by mol) (similar to that currently represented by JUUL) and amended with benzoic acid to give $C_{\mathrm{HA}}=3.38 \times 10^{-4} \mathrm{~mol} / \mathrm{mL}$ and nicotine at $C_{\mathrm{Nic}}=3.30 \times 10^{-4}$ $\mathrm{mol} / \mathrm{mL}\left(C_{\mathrm{HA}} / C_{\mathrm{Nic}}=1.03\right.$, nicotine $\left.4.6 \mathrm{wt} \%\right)$. To investigate the effects of water on nicotine protonation, an aliquot of the second mixture was amended with water at 5\% (by volume). 
Table 1. Listed vs. measured nicotine contents, molar acid/nicotine ratios, and free-base fraction $\left(\alpha_{\mathrm{fb}}\right)$ for a selection of JUUL pod liquids, 'look-a-like/knockoff' pod liquids, and bottled e-liquids (additional details can be found online in supplementary table $\mathrm{S}-1$ ).

\begin{tabular}{|c|c|c|c|c|c|}
\hline $\begin{array}{l}\text { Brand "Flavour"/Fig. } 2 \\
\text { Abbreviation }\end{array}$ & $\begin{array}{c}\text { Nicotine } \\
\text { Weight \%* } \\
\text { (Listed/Measd.) }\end{array}$ & $\begin{array}{c}\mathrm{Nic}_{\text {tot }} \\
\mathrm{mg} / \mathrm{mL} \\
\text { (Measd.) }\end{array}$ & Acidt & $\begin{array}{c}\text { Molar } \\
\text { Acid/Nicotine } \\
\text { Ratio }\left(C_{\mathrm{HA}} / C_{\mathrm{Nic}}\right)\end{array}$ & $\alpha_{\mathrm{fb}} \dagger$ \\
\hline \multicolumn{6}{|l|}{ JUUL "pods" (5\% } \\
\hline \multicolumn{6}{|l|}{$\frac{\text { nicotine by wt.) }}{\text { JUUL "Cool }}$} \\
\hline $\begin{array}{l}\text { Mint"/JUUL(8) group } \\
\text { JUUL "Classic }\end{array}$ & $5.0 / 5.1$ & 60 & BA & 0.97 & 0.13 \\
\hline $\begin{array}{l}\text { Menthol"/JUUL(8) group } \\
\text { JUUL "Crème }\end{array}$ & $5.0 / 4.9$ & 58 & BA & 0.98 & 0.13 \\
\hline $\begin{array}{l}\text { Brulee"/JUUL(8) group } \\
\text { JUUL "Fruit }\end{array}$ & $5.0 / 5.1$ & 60 & BA & 0.97 & 0.12 \\
\hline $\begin{array}{l}\text { Medley"/JUUL(8) group } \\
\text { JUUL "Cool }\end{array}$ & $5.0 / 5.0$ & 59 & BA & 0.99 & 0.12 \\
\hline \multicolumn{6}{|l|}{ Cucumber"/JUUL(8) } \\
\hline $\begin{array}{l}\text { group } \\
\text { JUUL "Classic }\end{array}$ & $5.0 / 5.0$ & 59 & BA & 1.00 & 0.11 \\
\hline $\begin{array}{l}\text { Tobacco"/JUUL(8) group } \\
\text { JUUL "Virginia }\end{array}$ & $5.0 / 5.0$ & 59 & BA & 1.00 & 0.11 \\
\hline $\begin{array}{l}\text { Tobacco"/JUUL(8) group } \\
\text { JUUL "Mango"/JUUL(8) }\end{array}$ & $5.0 / 5.1$ & 60 & BA & 1.00 & 0.11 \\
\hline $\begin{array}{l}\text { group } \\
\text { JUUL "pods" ( } 3 \%\end{array}$ & $5.0 / 5.2$ & 62 & BA & 0.99 & 0.09 \\
\hline \multicolumn{6}{|l|}{$\frac{\text { nicotine by wt.) }}{\text { JUUL "Virginia }}$} \\
\hline $\begin{array}{l}\text { Tobacco"/JUUL(2) group } \\
\text { JUUL "Mint"/JUUL(2) }\end{array}$ & $3.0 / 3.0$ & 35 & BA & 0.94 & 0.14 \\
\hline group & $3.0 / 3.0$ & 35 & BA & 1.04 & $\underline{0.11}$ \\
\hline
\end{tabular}

for 10 JUUL liquids: ave. $\pm 1 \mathrm{sd}: \quad 0.12 \pm 0.01$

\begin{tabular}{|c|c|c|c|c|c|}
\hline \multicolumn{6}{|l|}{ Other "nicotine salt" } \\
\hline Eon Smoke & & & & & \\
\hline $\begin{array}{l}\text { "Mango"/EM } \\
\text { Myle "Summer }\end{array}$ & $6.0 / 4.0$ & 47 & BA & 3.43 & $0.00 \S$ \\
\hline Strawberry"/SS & $5.0 / 4.3$ & 51 & BA & 1.02 & 0.09 \\
\hline ZiiP "Cappuccino"/ZiC & $5.0 / 3.3$ & 38 & BA & 4.03 & $0.01 \S$ \\
\hline ZiiP "Mango"/ZiM & $5.0 / 3.5$ & 41 & BA & 3.71 & $0.00 \S$ \\
\hline SMPO "Full Fruit"/FF & $5.0 / 2.3$ & 27 & BA & 0.76 & 0.15 \\
\hline ZOOR "Apple"/ZA & $5.0 / 4.5$ & 53 & LA & $0.22 \pi$ & $0.19 \S$ \\
\hline
\end{tabular}




\begin{tabular}{|c|c|c|c|c|c|}
\hline $\begin{array}{l}\text { Brand "Flavour"/Fig. } 2 \\
\text { Abbreviation }\end{array}$ & $\begin{array}{c}\text { Nicotine } \\
\text { Weight \%* } \\
\text { (Listed/Measd.) }\end{array}$ & $\begin{array}{c}\mathrm{Nic}_{\text {tot }} \\
\mathrm{mg} / \mathrm{mL} \\
\text { (Measd.) }\end{array}$ & Acid + & $\begin{array}{c}\text { Molar } \\
\text { Acid/Nicotine } \\
\text { Ratio }\left(C_{\mathrm{HA}} / C_{\text {Nic }}\right) \\
\end{array}$ & $\alpha_{\mathrm{fb}} \dagger$ \\
\hline ZOOR "Mint Ice"/ZMI & $5.0 / 4.4$ & 52 & LA & $0.29 \llbracket$ & 0.17 \\
\hline $\begin{array}{l}\text { ZOOR "Cake"/ZC } \\
\text { “Nicotine salt" bottled } \\
\frac{\text { e-liquids }}{\text { Pacha Mama Salts }}\end{array}$ & $5.0 / 4.8$ & 57 & LA & $0.25 \mathbb{I}$ & 0.14 \\
\hline $\begin{array}{l}\text { "Fuji"/Fuji-50 } \\
\text { Salt Bae }{ }^{50} \text { "Blue } \\
\text { Raspberry }\end{array}$ & $4.2 / 4.2$ & 50 & $U$ & $U$ & 0.08 \\
\hline $\begin{array}{l}\text { Lemonade"/BRL-50 } \\
\text { Pacha Mama Salts }\end{array}$ & $4.2 / 4.1$ & 49 & $U$ & $U$ & 0.01 \\
\hline $\begin{array}{l}\text { "Fuji"/Fuji-25 } \\
\text { Salt Bae }{ }^{50} \text { "Blue } \\
\text { Raspberry }\end{array}$ & $2.1 / 2.0$ & 24 & $U$ & $U$ & 0.08 \\
\hline $\begin{array}{l}\text { Lemonade"/BRL-25 } \\
\text { Non-"salt" bottled e- } \\
\frac{\text { liquids }}{\text { Fuzion Vapor }}\end{array}$ & $2.1 / 2.1$ & 25 & $U$ & $U$ & 0.02 \\
\hline $\begin{array}{l}\text { "Ectoplasm"/Ec-24 } \\
\text { Fuzion Vapor } \\
\text { "Roundhouse with }\end{array}$ & $2.0 / 1.0$ & 12 & - & $U$ & $0.98 \S$ \\
\hline $\begin{array}{l}\text { Cream"/RwC-24 } \\
\text { Fuzion Vapor "Unicorn }\end{array}$ & $2.0 / 2.8$ & 33 & $U$ & $u$ & 0.70 \\
\hline $\begin{array}{l}\text { Blood"/UB-24 } \\
\text { Fuzion Vapor }\end{array}$ & $2.0 / 0.9$ & 11 & $U$ & $U$ & 0.84 \\
\hline $\begin{array}{l}\text { "Ectoplasm"/Ec-6 } \\
\text { Fuzion Vapor "Unicorn }\end{array}$ & $0.5 / 0.5$ & 5 & - & U & 0.96 \\
\hline $\begin{array}{l}\text { Blood Prime"/UBP-6 } \\
\text { Fuzion Vapor "Unicorn }\end{array}$ & $0.5 / 0.4$ & 5 & $U$ & $U$ & 0.53 \\
\hline $\begin{array}{l}\text { Blood"/UB-6 } \\
\text { Fuzion Vapor } \\
\text { "Roundhouse with }\end{array}$ & $0.5 / 0.4$ & 5 & $U$ & $U$ & 0.43 \\
\hline Cream"/RwC-6 & $0.5 / 0.5$ & 6 & U & $U$ & 0.08 \\
\hline
\end{tabular}

* Calculated by integrating ${ }^{1} \mathrm{H}$ NMR resonances for nicotine relative to propylene glycol and glycerol resonances in each e- liquid and obtaining the mole per cent values, which were then converted into wt $\%$ values. These values do not reflect the variable presence of water, accounting for water affecting nicotine $\mathrm{mg} / \mathrm{mL}$ by less than $\sim 10 \%$.

$\uparrow$ Values by liquid chromatography for JUUL products, by NMR for all others. Ratio computed based on the main acid contributor for each liquid. 
¥Different chemical shift references were used based on composition. The value presented is that for the average at $40^{\circ} \mathrm{C}$. For details, see the Methods section.

$\S$ Only one $\alpha_{\mathrm{fb}}$ value was obtained by ${ }^{1} \mathrm{H}$ NMR due to either resonance overlap or peak broadening.

IIncomplete characterisation of the acid content.

ave., average; BA, benzoic acid; BRL-25, Salt Bae ${ }^{50}$ 'Blue Raspberry Lemonade'; BRL50, Salt Bae ${ }^{50}$ 'Blue Raspberry Lemonade'; $\mathrm{C}_{\mathrm{HA}}$, total molar- based concentrations of $\mathrm{HA}$; $\mathrm{C}_{\mathrm{Nic}}$, total molar- based concentrations of nicotine; Ec-6, Fuzion Vapour 'Ectoplasm'; Ec-24, Fuzion Vapor 'Ectoplasm'; EM, Eonsmoke 'Mango'; $\alpha \mathrm{fb}$, fraction of nicotine in the free- base form; FF, SMPO 'Full Fruit'; Fuji-25, Pacha Mama Salts 'Fuji'; Fuji-50, Pacha Mama Salts 'Fuji'; LA, levulinic acid; Nictot, total nicotine concentration; NMR, 1H NMR spectroscopy; RwC-6, Fuzion Vapour 'Roundhouse with Cream'; RwC-24, Fuzion Vapor 'Roundhouse with Cream'; SS, Myle 'Summer Strawberry'; U, unknown/undetected; UB-6, Fuzion Vapour 'Unicorn Blood'; UB-24, Fuzion Vapour 'Unicorn Blood'; UBP-6, Fuzion Vapour 'Unicorn Blood Prime'; wt\%, weight per cent; ZA, ZOOR 'Apple'; ZC, ZOOR 'Cake'; ZiC, ZiiP 'Cappuccino'; ZiM, ZiiP ‘Mango'; ZMI, ZOOR 'Mint Ice'.

Values of $K_{\mathrm{oa}, 1}$ were also calculated for benzoic acid at $40^{\circ} \mathrm{C}$ based on the data for the JUUL products in table 1 , assuming $C_{\mathrm{HA}} / C_{\mathrm{Nic}}=1$, as verified here by a liquid chromatography (LC) method discussed elsewhere. ${ }^{15}{ }^{1} \mathrm{H}$ NMR results gave slightly different $C_{\mathrm{HA}}: C_{\mathrm{Nic}}$ ratios (table S-1); however, NMR spectra can be subject to resonance overlap in these cases because of the presence of flavourants, so the LC-determined $C_{\mathrm{HA}} / C_{\mathrm{Nic}}$ ratios were used for the calculations herein.

$K_{\mathrm{oa}, 1}$ values were also determined for vanillin at $40^{\circ} \mathrm{C}$ in $45 / 55 \mathrm{PG} / \mathrm{GL}$ by weight (49/51 by mol). The mixture was amended with nicotine and three levels of vanillin. The three solutions were characterized by: (1) $C_{\mathrm{HA}}=1.80 \times 10^{-4}$ and $C_{\mathrm{Nic}}=3.61 \times 10^{-4}$ $\mathrm{mol} / \mathrm{mL}\left(C_{\mathrm{HA}} / C_{\mathrm{Nic}}=0.50\right)($ nicotine at $5.1 \mathrm{wt} \%),(2) C_{\mathrm{HA}}=3.67 \times 10^{-4}$ and $C_{\mathrm{Nic}}=3.59 \times$ 
$10^{-4} \mathrm{~mol} / \mathrm{mL}\left(C_{\mathrm{HA}} / C_{\mathrm{Nic}}=1.02\right)($ nicotine at $5.1 \mathrm{wt} \%)$, and (3) $C_{\mathrm{HA}}=5.15 \times 10^{-4}$ and $C_{\mathrm{Nic}}=$ $3.41 \times 10^{-4} \mathrm{~mol} / \mathrm{mL}\left(C_{\mathrm{HA}} / C_{\mathrm{Nic}}=1.51\right)($ nicotine at $4.9 \mathrm{wt} \%)$.

\subsubsection{JUUL Aerosol PM Determinations}

A fully charged JUUL device was equipped with a JUUL 'Classic Menthol' 5\% nicotine pod and vaped using the CORESTA puff method ( $55 \mathrm{~mL}$ puff volume, $3 \mathrm{~s}$ long) and employed vaping methods described previously. ${ }^{16} 17$ The JUUL device (+ e-liquid pod) was weighed before and after the generation of five puffs to obtain the mass of aerosol produced over the 5 puffs.

\subsection{Results}

\subsubsection{Protonation in E-liquids}

Table 1 lists the measured (by ${ }^{1} \mathrm{H}$ NMR) vs. manufacturer-listed nicotine concentrations and the measured $\alpha_{\mathrm{fb}}$ values (online supplementary figure S-3 visually depicts the data in a bar chart). The e-liquids tested included those for JUUL pods, other look-a-like/knockoff pods, bottled nicotine salt e-liquids, and early generation (ie, nonsalt) bottled e-liquids. The agreement between the listed and actual nicotine contents varied among brands; in this work, the measured values were used; online supplementary figure S-1 illustrates the differences among the e-liquids. Table 1 also gives $C_{\mathrm{HA}} / C_{\mathrm{Nic}}$; the acids were fully identifiable by NMR for the first 14 entries, and the presence of at least one acid was identified for the first 14 entries, and the presence of at least one acid was identified for the first 18 e-liquids. $C_{\mathrm{HA}} / C_{\mathrm{Nic}}$ values varied widely among the brands (see also online supplementary figure $S-2$ ). Online supplementary figure $S-5$ is a comparison 
of the ${ }^{1} \mathrm{H}$ NMR spectra for two e-liquids with differing ratios of benzoic acid relative to nicotine; for one, $C_{\mathrm{HA}} / C_{\mathrm{Nic}}=\sim, 1$ and for the other, $C_{\mathrm{HA}} / C_{\mathrm{Nic}}=\sim 4$.

Figure 2 is a plot of measured Nic $\mathrm{tot}_{\mathrm{t}} v s . \boldsymbol{\alpha}_{\mathrm{fb}}$. Lines of constant fb concentration as given by the product $\alpha_{\mathrm{fb}} \mathrm{Nic}_{\text {tot }}$ plot as hyperbolas (see also the issue cover graphic for Duell et al). ${ }^{1}$ All the e-liquids with $C_{\mathrm{HA}} / C_{\mathrm{Nic}} \approx 1$ with benzoic acid were found to be characterized by similar $\alpha_{\mathrm{fb}}$ values (0.09 to 0.14$)$. As noted earlier, the inhalation harshness of a nicotine aerosol is related to the fb concentration in the aerosol liquid, as given by $\alpha_{\mathrm{fb}} \mathrm{Nic}_{\text {tot. }}$ Values for $\alpha_{\mathrm{fb}} \mathrm{Nic}_{\text {tot }}$ can be computed from the data in table 1 (see also online supplementary figure S-4). Bookending these values, e-liquids with $C_{\mathrm{HA}} / C_{\mathrm{Nic}} \gg 1$ gave $\alpha_{\mathrm{fb}} \sim 0$, and some e-liquids that were not marketed as nicotine salts gave $\alpha_{\mathrm{fb}}$ values as high as 0.98 .

Besides carboxylic acids (eg, benzoic acid and levulinic acid) as protonating agents, the prevalent flavour phenols vanillin and ethyl vanillin can contribute to protonation of nicotine; these two weak acids can be found at high concentrations in some e-liquids. ${ }^{18}$ Such an effect on $\alpha_{\mathrm{fb}}$ may be indicated in the $\alpha_{\mathrm{fb}}$ values for the 'Roundhouse with Cream' flavour formulations for two different Nic tot $_{\text {values, }} 33$ and 6 $\mathrm{mg} / \mathrm{mL}$, with $\alpha_{\mathrm{fb}}=0.70$ and 0.08 , respectively. Assuming a constant phenol flavourant level, the lower $\alpha_{\mathrm{fb}}$ for the lower nicotine-level may have been caused in part by a higher total acids:nicotine ratio due to flavor chemicals. 


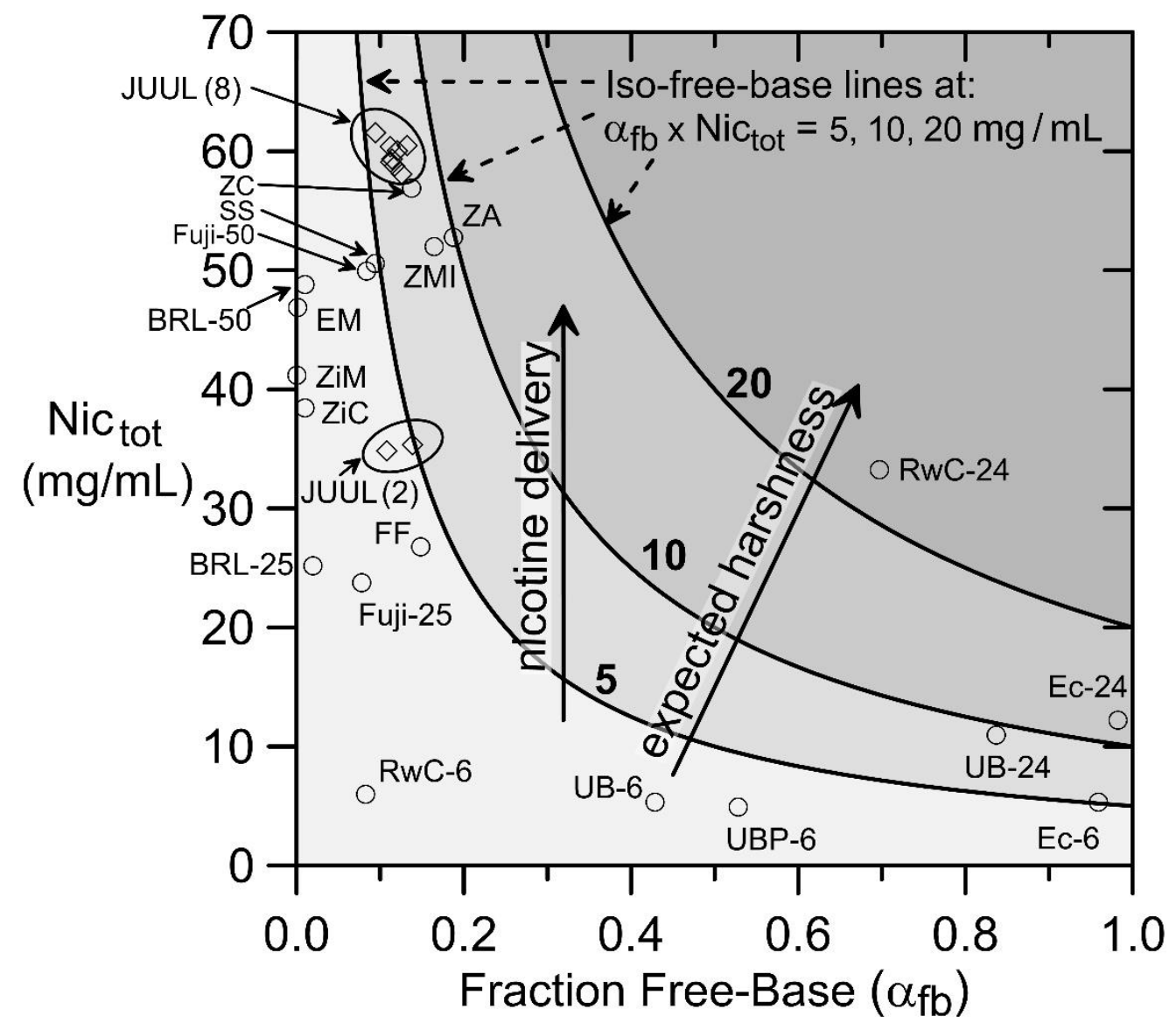

Figure 2. The expected harshness of a nicotine- containing product is influenced by both the free-base fraction $\left(\alpha_{\mathrm{fb}}\right)$ and the total nicotine concentration $\left(\mathrm{Nic}_{\mathrm{tot}}\right)$. BRL-25, SaltBae ${ }^{50}$ 'Blue Raspberry Lemonade'; BRL-50, SaltBae ${ }^{50}$ 'Blue Raspberry Lemonade'; Ec-6, Fuzion Vapour 'Ectoplasm'; Ec-24, Fuzion Vapor 'Ectoplasm'; EM, Eon Smoke 'Mango'; FF, SMPO 'Full Fruit'; Fuji-25, Pacha Mama Salts 'Fuji'; Fuji-50, Pacha Mama Salts 'Fuji'; Nic tot, total nicotine concentration; RwC-6, Fuzion Vapour 'Roundhouse with Cream'; RwC-24, Fuzion Vapor 'Roundhouse with Cream'; SS, Myle 'Summer Strawberry'; UB-6, Fuzion Vapour 'Unicorn Blood'; UB-24, Fuzion Vapour 'Unicorn Blood'; UBP-6, Fuzion Vapour 'Unicorn Blood Prime'; ZA, ZOOR ‘Apple'; ZC, ZOOR ‘Cake'; ZiC, ZiiP ‘Cappuccino’; ZiM, ZiiP ‘Mango’; ZMI, ZOOR ‘Mint Ice’. 


\subsection{2. $\quad K_{\mathrm{oa}, 1}$ Determinations}

The $K_{\mathrm{oa}, 1}$ values $\left(40^{\circ} \mathrm{C} \approx 37^{\circ} \mathrm{C}\right)$ obtained here are provided in table 2 . For benzoic acid, values were determined in JUUL liquids and in two laboratory-prepared mixtures (with added 1:1, by mol, nicotine:benzoic acid): 43/57 PG/GL and 32/68 PG/GL (similar to JUUL) by weight. The average $K_{\mathrm{oa}, 1}$ value for the JUUL e-liquids tested was 67 , which is in reasonable agreement with $K_{\mathrm{oa}, 1}$ for $43 / 57$ PG/GL by weight, where $K_{\mathrm{oa}, 1}=26$ and with $K_{\mathrm{oa}, 1}$ for $32 / 68 \mathrm{PG} / \mathrm{GL}$ by weight, where $K_{\mathrm{oa}, 1}=31$. When five vol $\%$ water was added to the $32 / 68 \mathrm{PG} / \mathrm{GL}$ (by weight) mixture, $K_{\mathrm{oa}, 1}=51$; this sample may be the most comparable to the JUUL liquids, contain some water. For vanillin in $~ 45 / 55$ PG/GL by weight, $K_{\mathrm{oa}, 1}$ averaged 0.0089 , about 6000 times smaller than for benzoic acid. (At constant $C_{\mathrm{Nic}}$, the $K_{\mathrm{oa}, 1}$ values for vanillin may indicate some tendency to increase with an increasing $C_{\mathrm{HA}}: C_{\mathrm{Nic}}$ ratio; an increasingly ionic medium would be expected to favor the $\mathrm{HA}+\mathrm{Nic}=\mathrm{A}^{-}+\mathrm{NicH}^{+}$reaction, due to Debye-Hückel effects.) 
Table 2. Values of $K_{\mathrm{oa}, 1}\left(40^{\circ} \mathrm{C}\right)$ for benzoic acid (BA) and vanillin in e-liquid formulations.

\begin{tabular}{lcccc}
\hline Benzoic Acid & $\underline{\underline{C}_{\mathrm{HA}} / \mathrm{C}_{\mathrm{Nic}}}$ & $\underline{\alpha_{\mathrm{fb}}}$ & $\underline{K_{\text {overall }}}$ & $\underline{\underline{L}}$ \\
\hline JUUL "Cool Mint" (5\% Nic) & $0.97^{+}$ & 0.13 & 58 & 1.77 \\
JUUL "Classic Menthol" (5\% Nic) & $0.98^{+}$ & 0.13 & 53 & 1.72 \\
JUUL "Crème Brulee" (5\% Nic) & $0.97^{+}$ & 0.12 & 72 & 1.86 \\
JUUL "Fruit Medley" (5\% Nic) & $0.99^{+}$ & 0.12 & 59 & 1.77 \\
JUUL "Cool Cucumber" (5\% Nic) & $1.00^{+}$ & 0.11 & 65 & 1.82 \\
JUUL "Classic Tobacco" (5\% Nic) & $1.00^{+}$ & 0.11 & 65 & 1.82 \\
JUUL "Virginia Tobacco" (5\% Nic) & $1.00^{+}$ & 0.11 & 65 & 1.82 \\
JUUL "Mango" (5\% Nic) & $0.99^{+}$ & 0.09 & 115 & 2.06 \\
JUUL "Virginia Tobacco" (3\% Nic) & $0.94^{+}$ & 0.14 & 66 & 1.82 \\
JUUL "Mint" (3\% Nic) & $\underline{1.04^{+}}$ & $\underline{0.11}$ & $\underline{48}$ & $\underline{1.68}$ \\
& $0.99 \pm 0.03$ & $0.12 \pm 0.01$ & $67 \pm 18$ & $1.81 \pm 0.10$
\end{tabular}

43/57 PG/GL (by wt.) + Nic + BA

(final Nic level $=4.6 \mathrm{wt} \%$ )

32/68 PG/GL (by wt.) + Nic + BA

(final Nic level $=4.6 \mathrm{wt} \%$ )

32/68 PG/GL (by wt.) + Nic + BA +

$5 \%$ (by vol.) water (final Nic level =

$4.5 \mathrm{wt} \%)$

Vanillin

45/55 PG/GL (by wt.) + Nic + vanillin

(final Nic level $=5.1 \%$ )

45/55 PG/GL (by wt.) + Nic +

vanillin

(final Nic level $=5.1 \%$ )

45/55 PG/GL (by wt.) + Nic + vanillin

(final Nic level $=4.9 \%$ )

$1.51 \quad 0.88 \quad \underline{0.0120} \quad \underline{-1.92}$

1.01

1.03

1.03

0.11

0.16

26

1.41

0.14

31

1.49

51

1.71

$\begin{array}{llll}0.50 & 0.95 & 0.0058 & -2.23\end{array}$

$\begin{array}{llll}1.02 & 0.91 & 0.0089 & -2.05\end{array}$

$$
\text { ave. } \pm 1 \mathrm{sd}: \begin{array}{cc}
0.0089 \pm & -2.07 \pm \\
0.0025 & 0.13
\end{array}
$$

${ }^{\dagger}$ By liquid chromatography (LC) for both nicotine and benzoic acid, using a method discussed elsewhere. ${ }^{15}$ 


\subsubsection{JUUL Aerosol PM Determinations}

The average mass lost per puff, for five puffs, was $4.4 \mathrm{mg}$, which when divided by the puff volume $(55 \mathrm{~mL})$ results in an average aerosol PM of $\sim 80 \mathrm{mg} / \mathrm{L}$, or $80 \times 10^{6}$ $\mu \mathrm{g} / \mathrm{m}^{3}$. This is only slightly greater than the high end of the range for tobacco cigarettes, 13 to $63 \times 10^{6} \mu \mathrm{g} / \mathrm{m}^{3} .{ }^{19}$

\subsection{Discussion}

\subsubsection{Past was Prologue: Vu - Tobacco Smoke}

The chemistry changes during the rapid evolution of e-cigarettes closely parallel the events that occurred during the centuries-long development of smoked tobacco. The tobacco that the English colony of Jamestown in Virginia exported to England beginning in 1619 was dark, 'air-cured' tobacco. Air curing occurs by slow drying (6-8 weeks) in ventilated barns. Air cured (aka 'dark', 'brun' and 'black' $)^{20} 21$ tobacco generally produces tobacco smoke that is much more basic than other tobacco types. ${ }^{20} 22$ Leaf sugars, which are precursors of tobacco-smoke organic acids, are generally lost during slow air curing; it is this loss that accounts for the relatively high proportions of $\mathrm{fb}$ nicotine in the smoke aerosol droplets from air-cured tobacco ${ }^{23}$ (figure 3). Regardless of smoke basicity/acidity, most tobacco smoke nicotine is in the smoke PM, distributed among the fb and protonated nicotine forms. ${ }^{10}$ 
Time Course of $\alpha_{\mathrm{fb}}$ Changes

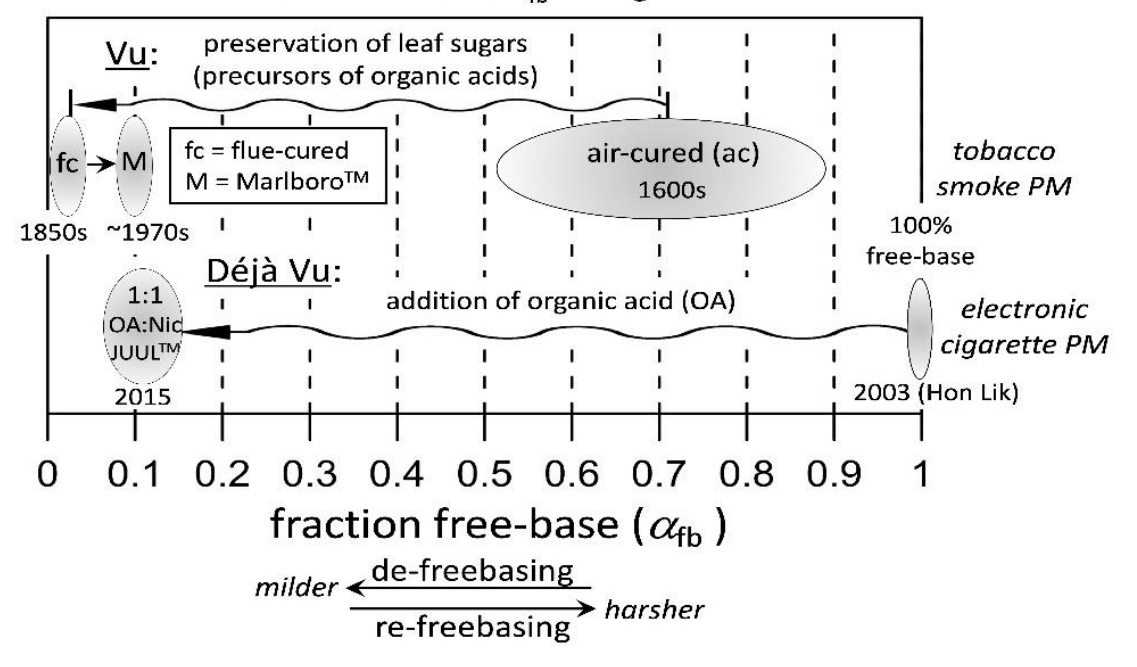

Figure 3. A visual representation of the historical changes in $\alpha_{\mathrm{fb}}$ in tobacco smoke $\mathrm{PM}^{27}$ (top) in comparison to how electronic cigarette fluids and their associated aerosols have been changed (bottom). fc, flue- cured; $\alpha_{\mathrm{fb}}$, fraction of nicotine in the free- base form; $\mathrm{M}$, Marlboro; Nic, nicotine; OA, organic acid; PM, particulate matter.

Nicotine-related harshness of tobacco smoke has long been viewed as being correlated with smoke basicity, with basicity favoring PM nicotine being in the volatilisable and therefore sensable (harsh) fb form. Consider:

'...The presence of unprotonated nicotine in the smoke of French cigarettes and the observation that French smokers of black tobacco inhale less frequently than smokers in England and the USA ... support our hypothesis that the pH is a determining factor in the "inhalability" of tobacco smoke., 20

'...increasing the $p H$... introduces a smoke with high physiological impact and a harsh bite, which would seem to offset the advantages gained from increased nicotine. ${ }^{24}$ 
'Flue-cured' (aka 'bright') tobacco was developed in the 1850s after the accidental discovery that rapid drying with high heat yields a bright yellow leaf that produces a noticeably milder smoke. ${ }^{25-27}$ Indeed, flue-cured tobacco remains high in leaf sugars so that the resulting smoke contains numerous organic acids. ${ }^{22}{ }^{27}$ While historical measurements of 'smoke $\mathrm{pH}$ ' both inside and outside the industry were indisputably flawed in absolute terms, within a given protocol (eg, the 'pH electrode' method), relative comparisons have likely been meaningful, so it is relevant that smoke $\mathrm{pH}$ was found by the industry to be strongly negatively correlated with both leaf sugar levels and leaf sugar/leaf nicotine ratios. ${ }^{28}$ In 1970 , Armitage and Turner ${ }^{29}$ wrote:

'It is usually believed that the, majority of cigarette smokers inhale to varying degrees the smoke which they take into their mouths, whereas the majority of cigar smokers do not.... One of the most striking differences between cigarette and cigar smoke is the pH of the smoke. The pH of T 29 cigarettes by the method of Grob...was 5.35, whereas the pH of the C 1 cigars was 8.5. ${ }^{29}$

Overall, as compared to tobacco smoke from air-cured tobacco, for flue-cured tobacco the fraction of the PM nicotine in the fb form is much lower. The role of acids in converting nicotine to a protonated, 'salt' form in tobacco smoke has long been understood. In 1909, Garner ${ }^{23}$ wrote:

'Apparently the only possible explanation of this pronounced effect on the sharpness of the smoke is that in the presence of the citric acid the nicotine enters the smoke in the form of a salt rather than in the free state, and 
thereby loses its pungency while still exerting the usual physiological effect. $^{23}$

Modern measurement of $\alpha_{\mathrm{fb}}$ values in cigarette smoke PM began $\sim 15$ years ago. ${ }^{3}$ ${ }^{30}$ In 'American blend' cigarettes, flue-cured tobacco dominates. Thus, in measurements with tobacco smoke PM from nine commercial brands of cigarettes sold in the U.S., Pankow et al. ${ }^{3}$ reported relatively low $\alpha_{\mathrm{fb}}$ values, ranging between $\sim 0.01$ (GPC) to $\sim 0.10$ (Marlboro). Two other, atypical commercial brands gave higher $\alpha_{\mathrm{fb}}$ values: Gauloises Brunes (relatively high in air-cured tobacco) at $\alpha_{\mathrm{fb}}=0.25$, and American Spirit/Maroon at $\alpha_{\mathrm{fb}}=0.36 \cdot{ }^{3}$ Overall, together with historical evidence, it can be concluded air-cured tobacco was characterized by very high $\alpha_{\mathrm{fb}}$ values ( $\geq 0.4$ and perhaps $\geq 0.5$ )

Figure 3 summarizes the main tobacco product development stages: (1) aerosol PM produced from smoked tobacco products in the early 1600s contained high levels of fb nicotine and so were harsh upon inhalation; the $\alpha_{\mathrm{fb}}$ in the PM was likely greater than 0.5 ; (2) flue-curing allowed retention of plant acids in the leaf, bringing $\alpha_{\mathrm{fb}}$ values in smoke PM to $\sim 0.01$ (very mild) (note here that Proctor has aptly commented that manufacturers of cigars giving high fb smoke might similarly make their products more inhalable by adding acids, a process which he has termed 'de-freebasing'):; (3) For Marlboro, by using additives and/or blend manipulation ${ }^{31} 32$ to accomplish a Goldilocks Principle solution (ie, not too harsh, not too mild), $\alpha_{\mathrm{fb}}$ was brought to $\sim 0.1$ for a tolerable/desired level of impact/harshness. Consider, by analogy, human affinity for the sensory 'bite' of carbonated beverages. ${ }^{33}$ Much has been written on the technical efforts 
of Philip Morris and its competitors to understand and provide some nicotine 'impact'.31

32 Overall, the tobacco smoke trajectory was $\alpha_{\mathrm{fb}} \geq 0.5 \rightarrow \sim 0 \rightarrow \sim 0.1$.

\subsubsection{Present: 'Déjà Vu' - E-cigarette Aerosols}

Stepanov and Fujioka ${ }^{34}$ where the first to consider the acid/base chemistry of nicotine in e-liquids. Most early versions of e-cigarettes used PG/GL-based fluids with total nicotine levels of 6 to $24 \mathrm{mg} / \mathrm{mL}$ and $\alpha_{\mathrm{fb}} \approx 1$ (nicotine + PG/GL is characterized by $\left.\alpha_{\mathrm{fb}} \approx 1\right) .{ }^{16}$ It has been verified that such e-liquids correspondingly generate e-cigarette aerosol PM with $\alpha_{\mathrm{fb}} \approx 1 .{ }^{16}$ When e-liquids including some acid and their resulting aerosol PM are compared, total nicotine levels have been found to be similar, ${ }^{135}$ as have the $\alpha_{\mathrm{fb}}$ values. ${ }^{1}$ Following our prior work, ${ }^{1}$ the product $\alpha_{\mathrm{fb}}$ Nic ${ }_{\text {tot }}$ can be used to compare eliquid fb delivery values, with JUUL products having been found to be de-freebased to $\alpha_{\mathrm{fb}} \approx 0.1$.

Cigarette smoke PM generally contains nicotine levels that are much higher than those in early e-liquids. Assuming unit density for cigarette smoke PM, the values of $\sim 54$ $\mathrm{mg} / \mathrm{mL}$ for the GPC brand, and $72 \mathrm{mg} / \mathrm{mL}$ for Marlboro ('red') have been reported. ${ }^{3}$ If ecigarettes were to attempt cigarette-like nicotine levels along with $\alpha_{\mathrm{fb}} \approx 1$, then with $\alpha_{\mathrm{fb}} \mathrm{Nic}_{\text {tot }} \approx 50$ to $70 \mathrm{mg} / \mathrm{mL}$, the aerosol would be expected to be exceedingly harsh upon inhalation. Enter JUUL, which was launched in 2015, offering its nicotine + benzoic acid pods $(5 \%(\mathrm{w} / \mathrm{w})$ nicotine, $\sim 59 \mathrm{mg} / \mathrm{mL})$; table 1 (and the results of Pankow et $a l^{15}$ ) indicate $\mathrm{a} \approx 1: 1$ molar ratio of benzoic acid to nicotine. As indicated earlier for $K_{\mathrm{oa}, 1}=$ 38.5 (table 1), equation (8) then gives $\alpha_{\mathrm{fb}}=0.14$ (see therefore figure 3 ), so that $\alpha_{\mathrm{fb}} \mathrm{Ni}_{\mathrm{ctot}}$ 
$\approx 8.3 \mathrm{mg} / \mathrm{mL}$. This is very similar to what has been found for Marlboro cigarettes $\left(\alpha_{\mathrm{fb}} \mathrm{Nic}_{\mathrm{tot}} \approx 0.10 \times 72 \mathrm{mg} / \mathrm{mL}=7.2 \mathrm{mg} / \mathrm{mL}\right){ }^{3}$ The trajectory for e-cigarettes has then been a partial de-freebasing according to $\alpha_{\mathrm{fb}}=\sim 1 \rightarrow \sim 0.1$ (as compared to $\alpha_{\mathrm{fb}} \geq 0.5 \rightarrow$ $\sim 0 \rightarrow \sim 0.1$ for cigarettes). Thus, taken with the PM results discussed earlier, the JUUL design characteristics provide effective cigarette-like delivery of nicotine, including (1) high total nicotine concentration in the liquid ( $\left.\mathrm{Nic}_{\text {tot }}, \mathrm{mg} / \mathrm{mL}\right)$; (2) low but not zero fb fraction $\left(\alpha_{\mathrm{fb}}\right)$; (3) cigarette-like concentrations of fb nicotine in the aerosol droplets $\left(\alpha_{\mathrm{fb}} \mathrm{Nic}_{\mathrm{tot}}, \mathrm{mg} / \mathrm{mL}\right)$; and (4) relatively low, cigarette-like PM; along with (5) optional flavors and no tobacco-smoke odor: a flavoured (at present) e-cigarette analog of Marlboro.

The trajectory in figure 3 for smoked tobacco allowed cigarettes to become much more addictive, abused, and deadly than would have been the case if smoked tobacco remained of an air-cured type. The evolution of e-cigarettes has followed a similar overall trajectory. It is undoubtedly true that this evolution has made e-cigarettes more effective as substitutes to get smokers off combustibles. However, exactly as occurred with smoked tobacco, this evolution has likely made e-cigarette products vastly more addictive for never-smokers. The full public health implications of widely prevalent ecigarette use will only become fully apparent perhaps a decade hence. 


\subsection{What This Paper Adds}

- The chemistry of nicotine in aerosols from smoked tobacco and electronic cigarette products underlie their parallel product developments and popularities, and therefore their abuse liabilities.

- The development over more than four centuries of smoked tobacco products (defreebasing then partial re-freebasing) is compared with the development of ecigarette products during the last 16 years (extensive but incomplete defreebasing). An explanation is provided of what has been perceived by some as inconsistent that: (1) tobacco companies during the mid-20 $20^{\text {th }}$ century were interested in increasing the value of the free-base nicotine fraction (by the partial re-freebasing step) in the products' smoke aerosol particulate matter, denoted $\alpha_{\mathrm{fb}}$, while (2) some e-cigarette manufacturers have moved to decrease it (by the extensive but incomplete de-freebasing).

- Values of $\alpha_{\mathrm{fb}}$ are measured by ${ }^{1} \mathrm{H}$ nuclear magnetic resonance spectroscopy for a total of 29 products, including JUUL, JUUL look-a-like/knockoff products, as well as bottles of 'nicotine salt' and 'non-salt' e-liquids.

- The overall trajectory of smoked tobacco development is discussed as having been $\alpha_{\mathrm{fb}} \geq 0.5 \rightarrow \sim 0.02 \rightarrow \sim 0.1$. A 'Déjà Vu' trajectory of $\alpha_{\mathrm{fb}} \approx 1 \rightarrow \sim 0.1$ has been followed in the design of the nicotine-containing liquids used in e-cigarettes, as supported by the measurements of $\alpha_{\mathrm{fb}}$. 
- A mathematical framework and equilibrium chemistry model are developed for understanding nicotine protonation chemistry in e-cigarette fluids in terms of $K_{\mathrm{oa}, 1}$, the first overall nicotine protonation constant.

- De-freebasing has undoubtedly made e-cigarettes more effective as substitutes to get smokers off combustibles. However, as with smoked tobacco, it is likely that e-cigarettes have also been made vastly more addictive for never-smokers. The full public health implications of widely prevalent e-cigarette use will only become fully apparent perhaps a decade hence. 


\subsection{References}

1 Duell AK, Pankow JF, Peyton DH. Free-Base Nicotine Determination in Electronic Cigarette Liquids by ${ }^{1} \mathrm{H}$ NMR Spectroscopy. Chem Res Toxicol 2018;31:431-4.

2 Morie GP. Fraction of protonated and unprotonated nicotine in tobacco smoke at various pH values. Tobacco Sci 1972;16.

3 Pankow JF, Tavakoli AD, Luo W, et al. Percent free base nicotine in the tobacco smoke particulate matter of selected commercial and reference cigarettes. Chem Res Toxicol 2003;16:1014-8.

4 Krishnan- Sarin S, Jackson A, Morean M, et al. E-cigarette devices used by high-school youth. Drug Alcohol Depend 2019;194:395-400.

5 Ramamurthi D, Chau C, Jackler RK. JUUL and other stealth vaporisers: hiding the habit from parents and teachers. Tob Control 2018. doi:10.1136/ tobaccocontrol-2018054455. [Epub ahead of print: 15 Sep 2018].

6 Gentzke AS, Creamer M, Cullen KA, et al. Vital signs: tobacco product use among Middle and high school students - United States, 2011-2018. MMWR Morb Mortal Wkly Rep 2019;68:157-64.

7 La Vito A. Juul asks regulators to stop 18 companies from selling lookalike ecigarettes, 2018. Available: https://www.cnbc.com/2018/10/03/juul-asks-regulatorstostop-companies-selling-lookalike-e-cigarettes.html [Accessed 23 Sep 2019].

8 Barsanti KC, Luo W, Isabelle LM, et al. Tobacco smoke particulate matter chemistry by NMR. Magn Reson Chem 2007;45:167-70.

9 Pankow JF, Barsanti KC, Peyton DH. Fraction of free-base nicotine in fresh smoke particulate matter from the Eclipse "cigarette" by ${ }^{1} \mathrm{H}$ NMR spectroscopy. Chem Res Toxicol 2003;16:23-7.

10 Pankow JF. A consideration of the role of gas/particle partitioning in the deposition of nicotine and other tobacco smoke compounds in the respiratory tract. Chem Res Toxicol 2001;14:1465-81.

11 Barlow RB, Hamilton JT. Effects of $\mathrm{pH}$ on the activity of nicotine and nicotine monomethiodide on the rat diaphragm preparation. Br J Pharmacol Chemother 1962;18:543-9.

12 Clayton PM, Vas CA, Bui TTT, et al. Spectroscopic studies on nicotine and nornicotine in the UV region. Chirality 2013;25:288-93.

13 Pankow JFet al. Aquatic chemistry concepts. 2nd edn. Boca Raton, FL: Taylor and Francis, 2019.

14 Christensen JJ, Hansen LD. Handbook of proton ionization heats and related thermodynamic quantities. New York: J. Wiley and Sons, 1976. 
15 Pankow JF, Kim K, McWhirter KJ, et al. Benzene formation in electronic cigarettes. PLoS One 2017;12:e0173055.

16 Duell AK, McWhirter KJ, Korzun T, et al. Sucralose-enhanced degradation of electronic cigarette liquids during Vaping. Chem Res Toxicol 2019;32:1241-9.

17 Duell AK, Pankow JF, Gillette SM, et al. Boiling points of the propylene glycol + glycerol system at 1 atmosphere pressure: $188.6-292{ }^{\circ} \mathrm{C}$ without and with added water or nicotine. Chem Eng Commun 2018;205:1691-700.

18 Omaiye EE, McWhirter KJ, Luo W, et al. High concentrations of flavor chemicals are present in electronic cigarette refill fluids. Sci Rep 2019;9:2468.

19 Pankow JF. Calculating compound dependent gas-droplet distributions in aerosols of propylene glycol and glycerol from electronic cigarettes. J Aerosol Sci 2017;107:9-13.

20 Brunnemann KD, Hoffmann D. The pH of tobacco smoke. Food Cosmet Toxicol 1974;12:115-24.

21 De Stefani E, Fierro L, Barrios E. Black (air-cured) and blond (flue-cured) tobacco and cancer risk III: oesophageal cancer. Eur J Cancer 1993;29:763-6.

22 Elson LA, Betts TE, Passey RD. The sugar content and the $\mathrm{pH}$ of the smoke of cigarette, cigar and pipe tobaccos in relation to lung cancer. Int J Cancer 1972;9:666-75.

23 Garner WW. The Relation of Nicotine to the Burning Quality of Tobacco, Bureau of Plant Industry. In: Bureau of plant industry - Bulletin no 141, miscellaneous papers, 1909: 5-16.

24 Chen L. pH of smoke: a review, report number N-170, internal document of Lorillard tobacco company, 18 PP, Bates NOS. 00118164/8181. Available:

https://www.industrydocumentslibrary.ucsf.edu/ tobacco/docs/\# id=ykhn01011976 [Accessed 1 May 2019].

25 Caswell County Genealogy. Slade, Abisha b.15 SEP 1799, Caswell County genealogy. Available:

https://caswellcountync.org/genealogy/getperson.php?personID=I2238\&tree=tree 1 [Accessed 25 Jun 2019].

26 Caswell County Hist Association, from the Caswell County Historical Association. Bright leaf tobacco process, 2010. Available: http://ncccha.blogspot.com/ 2010/ 01/bright-leaf-tobacco-process.html [Accessed 14 May 2019].

27 Proctor RN. Golden holocaust: origins of the cigarette catastrophe and the case for abolition. Berkeley and Los Angeles, California: University of California Press, 2012.

28 Correlation of maximum smoke $\mathrm{pH}$ of Flue-cured tobacco with leaf and smoke constituents, Bates number 504428668-504428671. Available: https://www.industrydocuments.ucsf.edu/docs/\# id=yqbg00081977 [Accessed 1 May 2019]. 
29 Armitage AK, Turner DM. Absorption of nicotine in cigarette and cigar smoke through the oral mucosa. Nature 1970;226:1231-2.

30 Watson CH, Trommel JS, Ashley DL. Solid-Phase microextraction-based approach to determine free-base nicotine in trapped mainstream cigarette smoke total particulate matter. J Agric Food Chem 2004;52:7240-5.

31 Kessler D. The control and manipulation of nicotine and cigarettes (21 June 1994 testimony before the Subcommittee on health and the environment, Committee on energy and Commerce, us house of representatives). Tob Control 1994;3:362-9.

32 Stevenson T, Proctor RN. The secret and soul of Marlboro: Phillip Morris and the origins, spread, and denial of nicotine freebasing. Am J Public Health 2008;98:1184-94.

33 Wise PM, Wolf M, Thom SR, et al. The influence of bubbles on the perception carbonation bite. PLoS One 2013;8:e71488.

34 Stepanov I, Fujioka N. Bringing attention to e-cigarette $\mathrm{pH}$ as an important element for research and regulation: Table 1. Tob Control 2015;24:413-4.

35 El- Hellani A, Salman R, El- Hage R, et al. Nicotine and carbonyl emissions from popular electronic cigarette products: correlation to liquid composition and design characteristics. Nicotine Tob Res 2018;20:215-23. 
5.8. Acknowledgments

We thank Dr. Wentai Luo and Kevin McWhirter for their assistance with secondary HPLC verification of the nicotine:benzoic acid ratio in JUUL products.

5.9. Author Information

5.9.1. Affiliations

Department of Chemistry, Portland State University, Portland, Oregon 97207-0751, USA

Anna K. Duell, David H. Peyton, \& James F. Pankow

Department of Civil and Environmental Engineering, Portland State University, Portland, Oregon 97207-0751, USA

James F. Pankow

\subsubsection{Contributors}

AKD carried out the work; JFP conceived the work; and DHP directed the project. All authors contributed to the writing of the manuscript.

\subsubsection{Funding}

This work was supported by the U.S. National Institutes of Health, grant R01ES025257. Research reported was supported by the NIEHS and FDA Center for Tobacco Products (CTP). 


\subsubsection{Disclaimer}

This content is solely the responsibility of the authors and does not necessarily represent the view of the NIH or the FDA. The quote in the title has been attributed to Yogi Berra.

\subsubsection{Competing Interests}

The authors declare no competing interests.

5.9.6. Patient Consent for Publication

Not required.

\subsubsection{Provenance and Peer Review}

Not commissioned; externally peer reviewed.

\subsubsection{Data Availability Statement}

All data relevant to the study are included in the article or uploaded as supplementary information.

\subsubsection{Open Access}

This is an open access article distributed in accordance with the Creative Commons Attribution Non Commercial (CC BY- NC 4.0) license, which permits others to distribute, remix, adapt, build upon this work non- commercially, and license their derivative works on different terms, provided the original work is properly cited, appropriate credit is given, any changes made indicated, and the use is non- commercial. See: http:// creativecommons. org/ licenses/ by- nc/ 4. $0 /$.

\subsubsection{Corresponding Author}

Correspondence to David H. Peyton (peytond@pdx.edu). 
5.10. Supporting Information: Nicotine in Tobacco Product Aerosols: "It's Déjà Vu All Over Again"§

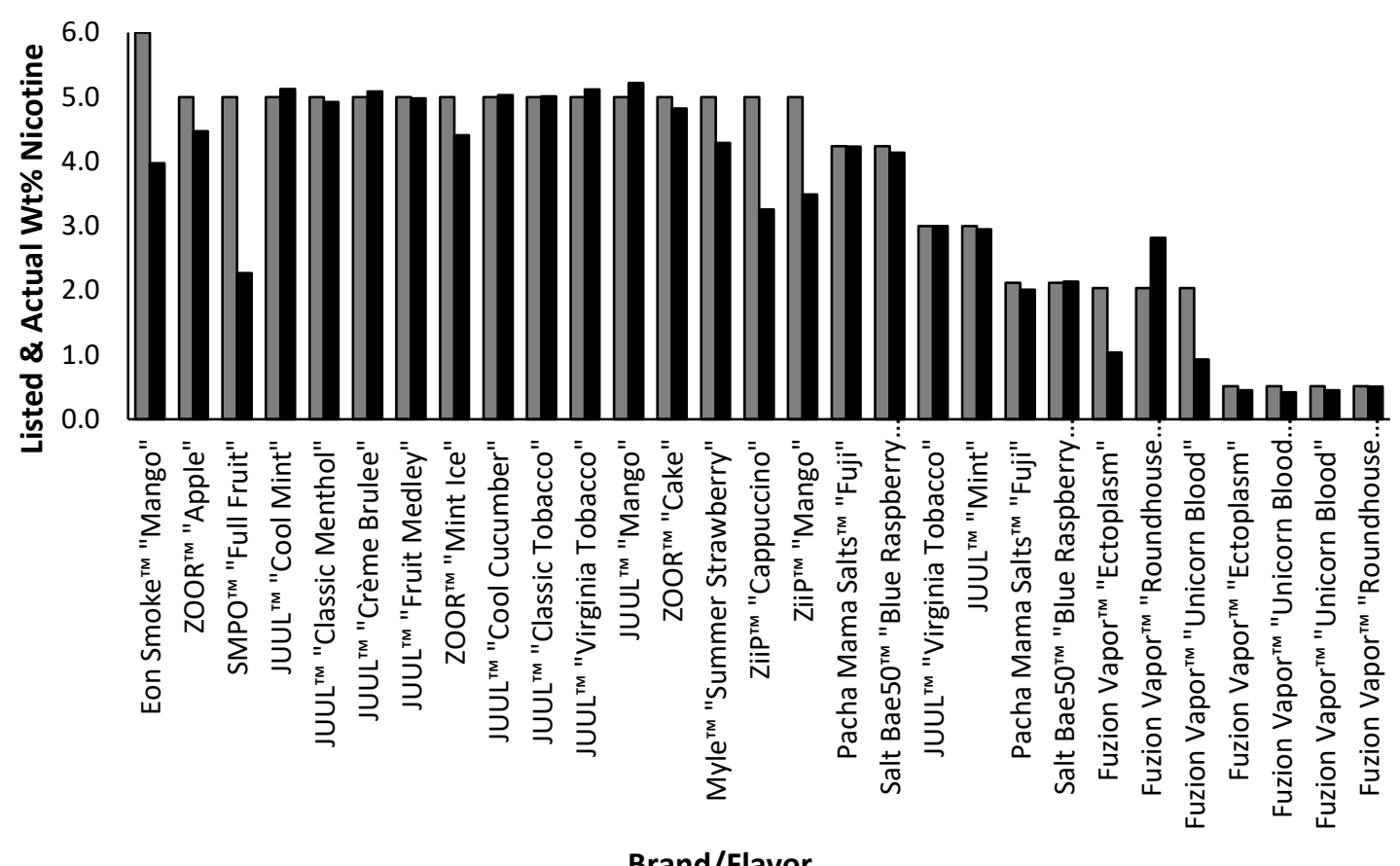

Figure S-1. Comparisons between listed and actual nicotine weight-\% contents for a selection of e-liquids. The listed amounts are shown by grey bars, while the actual amounts (as assessed by ${ }^{1} \mathrm{H}$ NMR integrations converted to weight-\% values) are shown by black bars. 


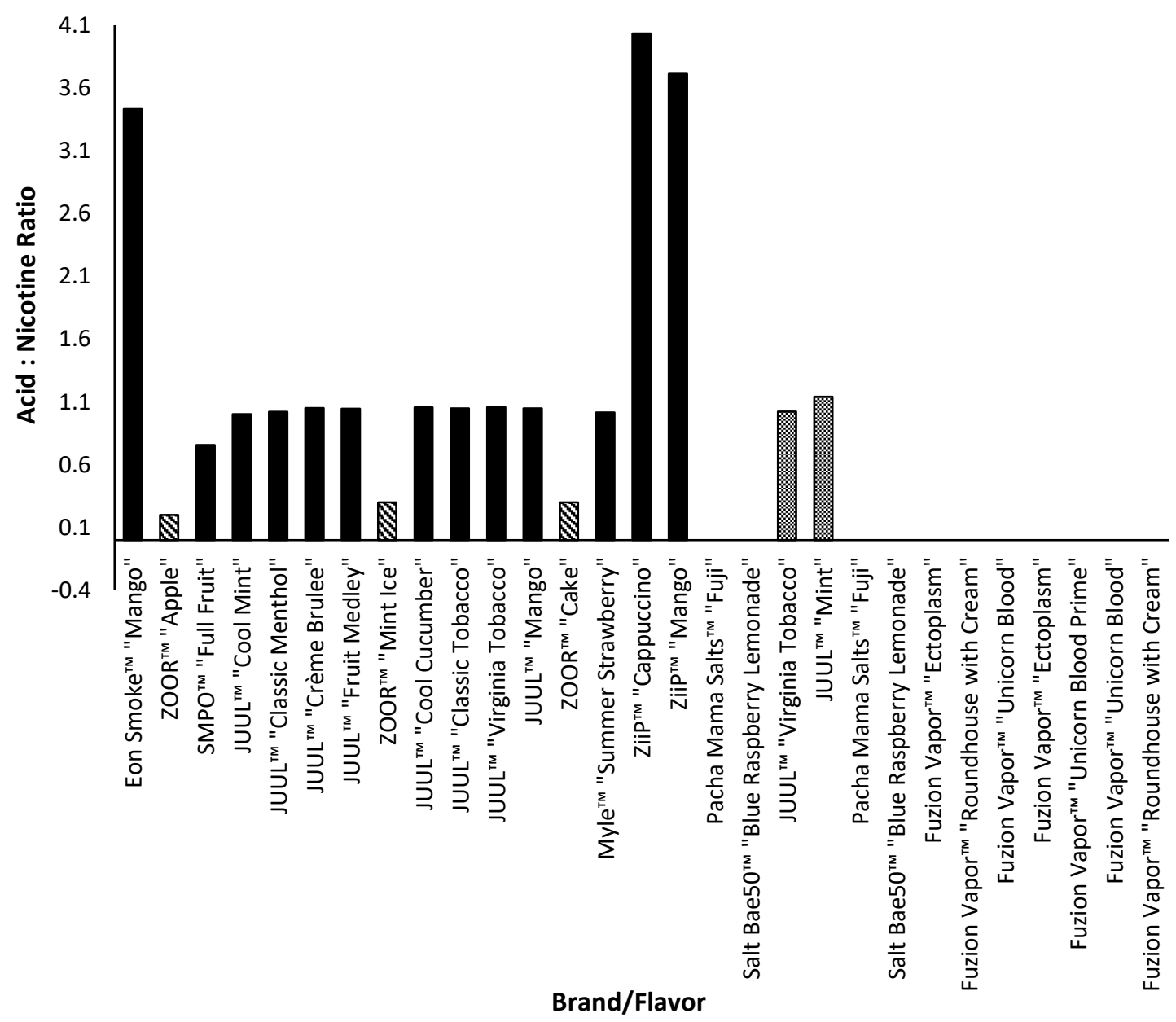

Figure S-2. The ratio of mol- $\%$ acid / mol- $\%$ nicotine for e-liquids containing benzoic acid or levulinic acid. Note that the ZOOR product was found not contain any benzoic acid (but did contain levulinic acid; indicated as striped bar), all other products with a positive acid/nicotine ratio contained benzoic acid, and the JUUL products are all essentially 1:1 benzoic acid/nicotine. JUUL 3 weight\% e-liquids are shown as checkered bars. 


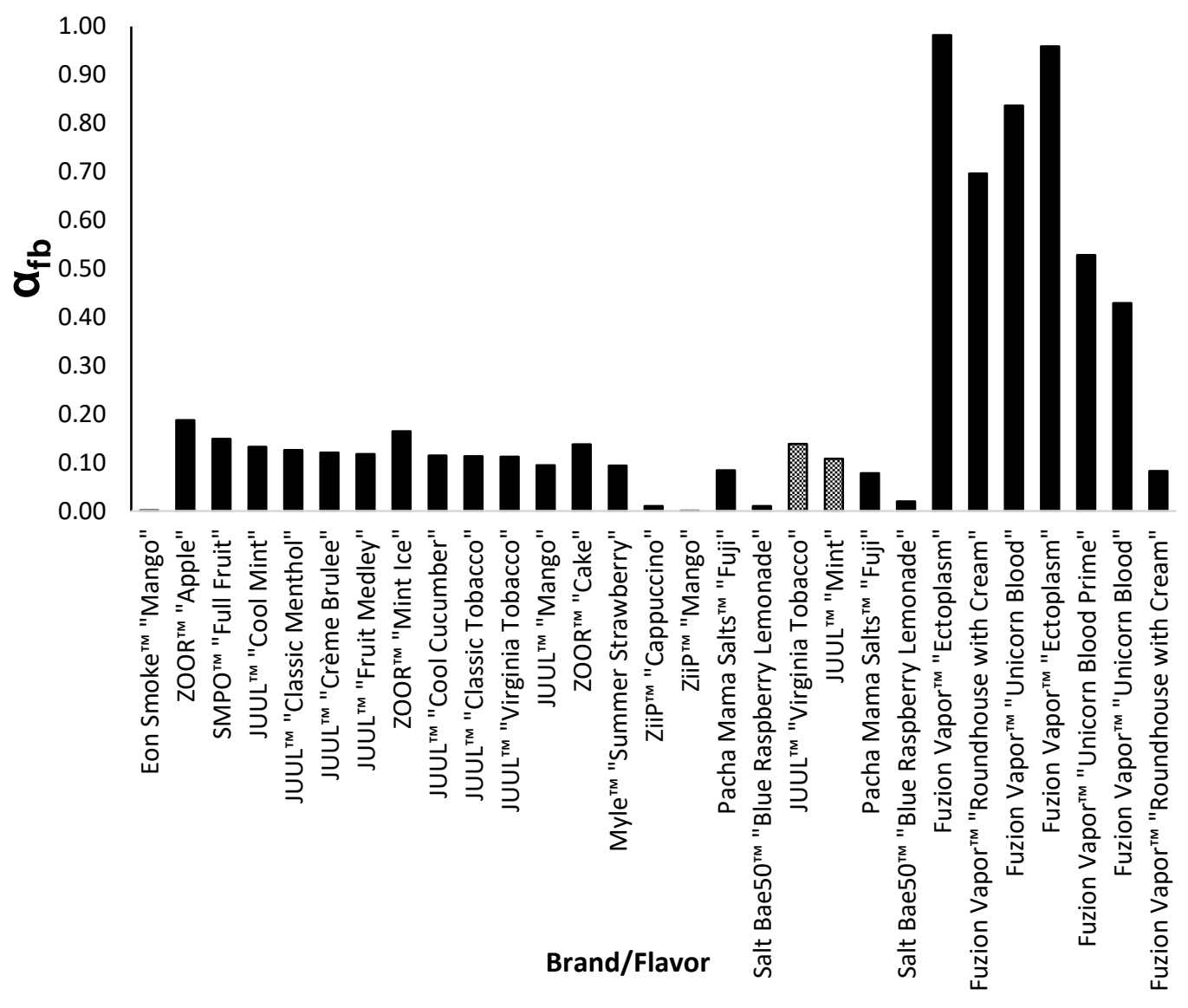

Figure S-3. The $\alpha_{\mathrm{fb}}$ values for the selection of e-liquids sampled. The two products from JUUL with 3 weight- $\%$ nicotine, as opposed to with 5 weight- $\%$ in the rest of the JUUL products are shown as checkered bars. These values are the averages (when possible) of the determinations available from the multiple aromatic resonances from the nicotine molecules, as explained in Materials and Methods. For some products, only one, rather than two, $\boldsymbol{\alpha}_{\mathrm{fb}}$ value was able to be calculated (See Table S-1). Note how these values correlate strongly with the inverse of the benzoic acid / nicotine ratios shown in Figure 2. 


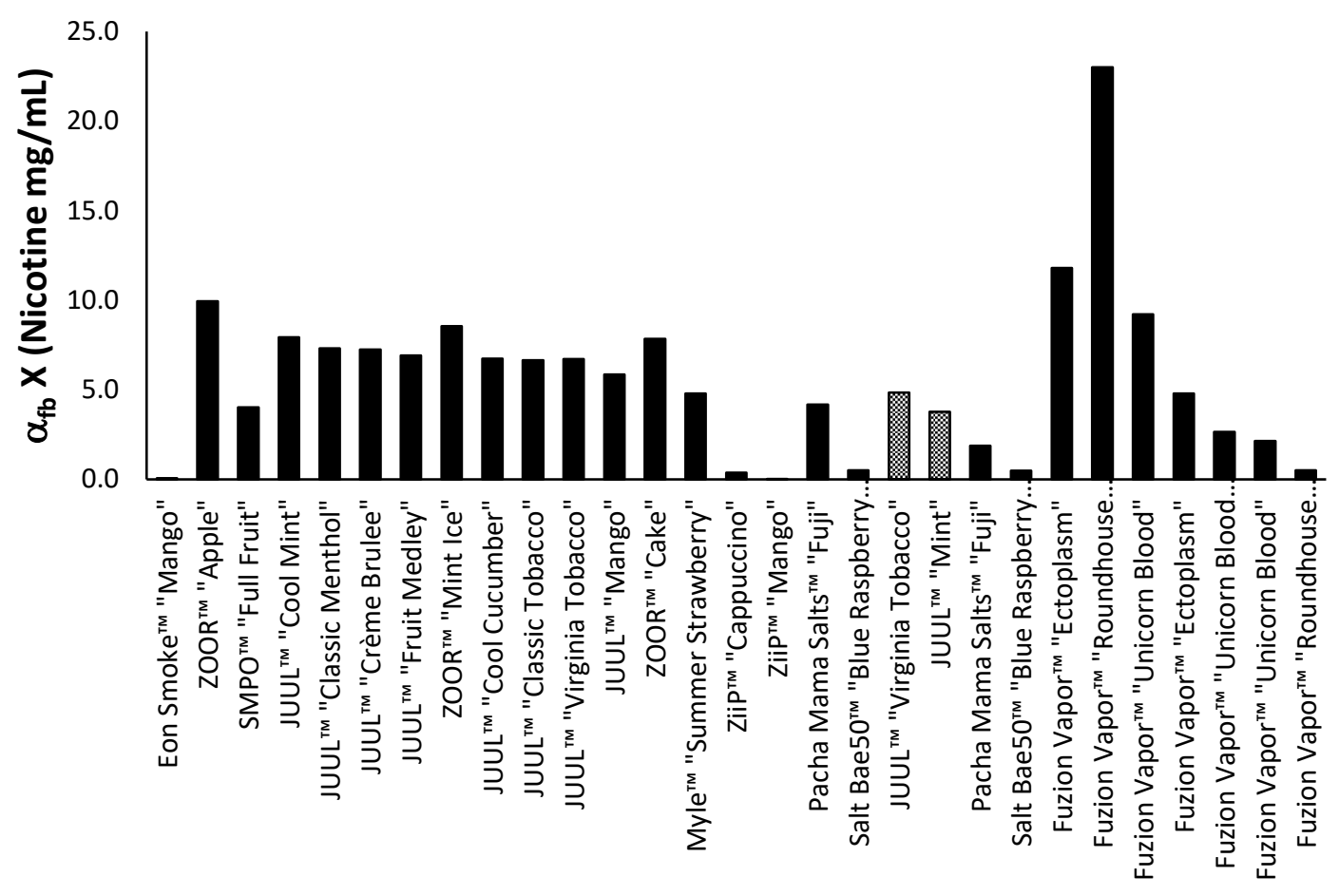

Brand/Flavor

Figure S-4. The $\alpha_{\mathrm{fb}}$ values from Figure 3, multiplied by the nicotine $\mathrm{mg} / \mathrm{mL}$ values. The resulting numbers permit a rough comparison of the total free-base nicotine exposure that a user would obtain from vaping the same weight of aerosol from each product. Note that the benzoic acid variation across these pods has a strong influence on the total exposure to free-base nicotine. The two products from JUUL with 5 weight- $\%$ nicotine, as opposed to with 3 weight- $\%$ in the rest of the JUUL products are shown as checkered bars. 

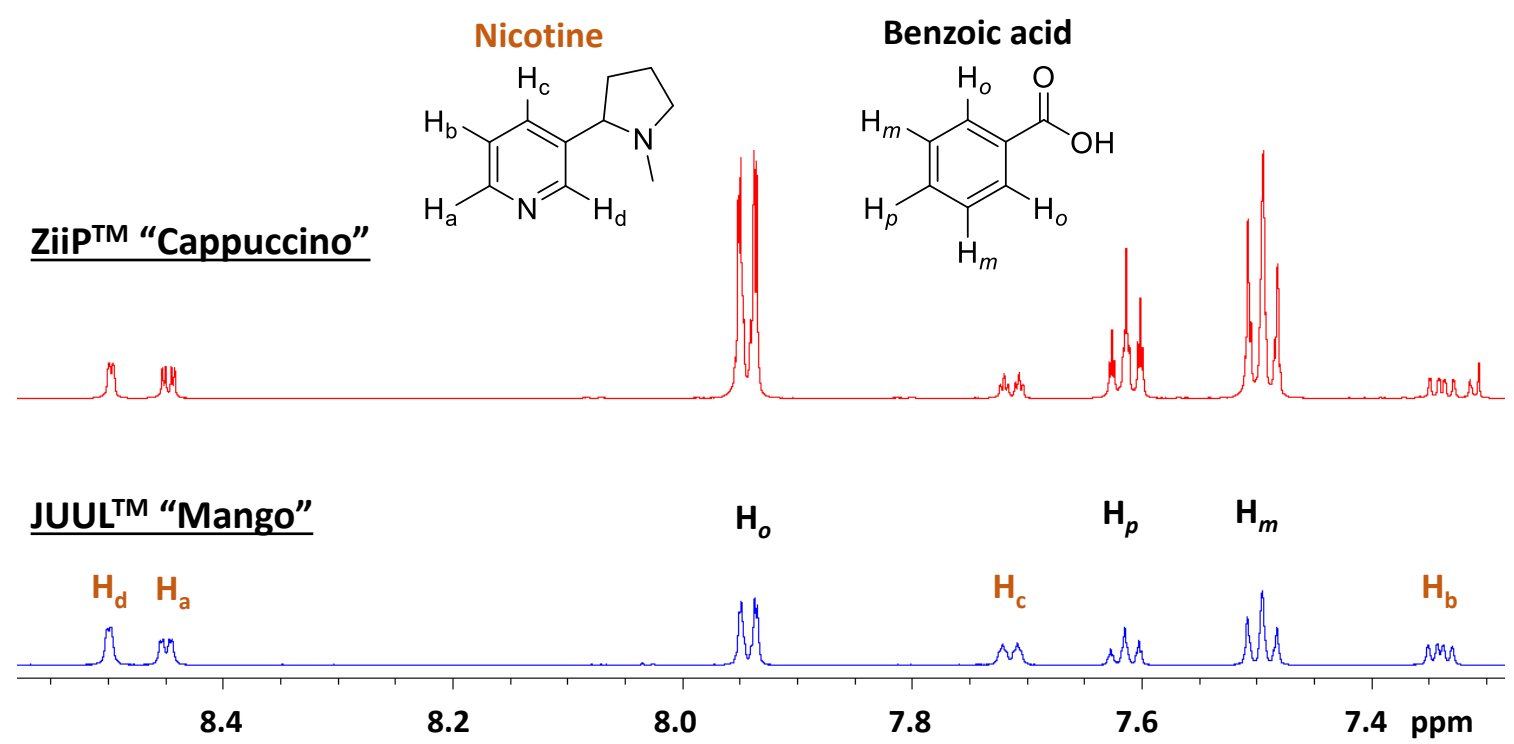

Figure S-5. Samples for composition analysis were prepared by adding a drop of each eliquid to DMSO- $d_{6}$ and testing by ${ }^{1} \mathrm{H}$ NMR. Spectra were normalized relative to the nicotine resonances because the e-liquids contained different ratios of propylene glycol:glycerol. Here, it can be seen that the ZiiP "Cappuccino" e-liquid has a much higher benzoic acid:nicotine ratio (4:1) than the JUUL "Mango" benzoic acid:nicotine ratio (1.1:1). 
Table S-1. Full table of values for listed vs. measured nicotine contents, molar acid/nicotine ratios (all determined by ${ }^{1} \mathrm{H}$ NMR), and free-base fraction determinations $\left(\alpha_{\mathrm{fb}}\right)$ for a selection JUUL pod liquids, "look-a-like/knockoff" pod liquids, and bottled e-liquids.

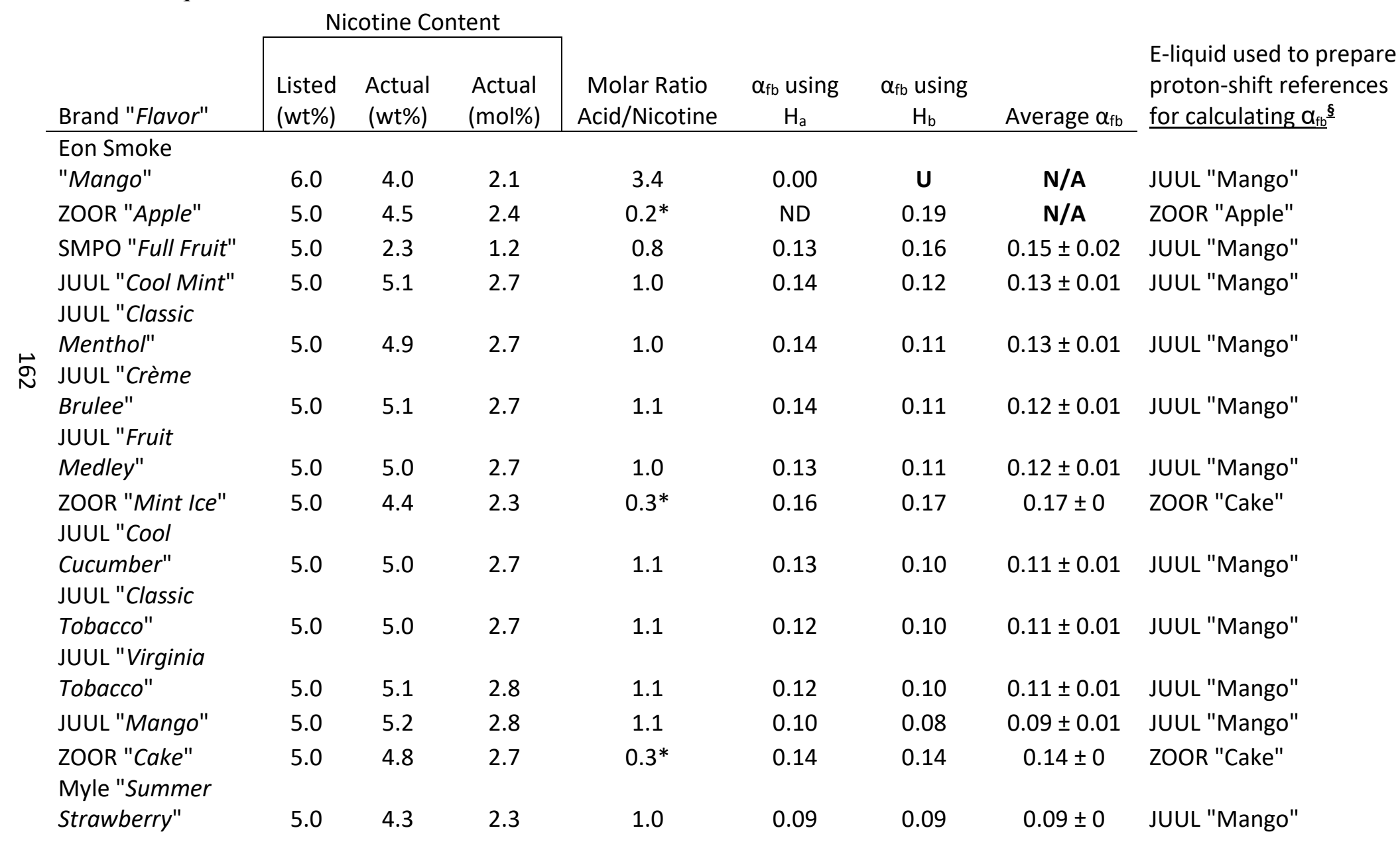




\begin{tabular}{|c|c|c|c|c|c|c|c|c|}
\hline \multirow[b]{2}{*}{ Brand "Flavor" } & \multicolumn{3}{|c|}{ Nicotine Content } & \multirow[b]{2}{*}{$\begin{array}{c}\text { Molar Ratio } \\
\text { Acid/Nicotine }\end{array}$} & \multirow[b]{2}{*}{$\begin{array}{c}\alpha_{\mathrm{fb}} \text { using } \\
\mathrm{H}_{\mathrm{a}}\end{array}$} & \multirow[b]{2}{*}{$\begin{array}{c}\alpha_{\mathrm{fb}} \text { using } \\
\mathrm{H}_{\mathrm{b}}\end{array}$} & \multirow[b]{2}{*}{ Average $\alpha_{\mathrm{fb}}$} & \multirow[b]{2}{*}{$\begin{array}{l}\text { E-liquid used to prepare } \\
\text { proton-shift references } \\
\text { for calculating } \alpha_{\mathrm{fb}} \underline{\S}\end{array}$} \\
\hline & $\begin{array}{l}\text { Listed } \\
\text { (wt\%) }\end{array}$ & $\begin{array}{l}\text { Actual } \\
\text { (wt\%) }\end{array}$ & $\begin{array}{l}\text { Actual } \\
\text { (mol\%) }\end{array}$ & & & & & \\
\hline ZiiP "Cappuccino" & 5.0 & 3.3 & 1.7 & 4.0 & 0.01 & $U$ & N/A & JUUL "Mango" \\
\hline $\begin{array}{l}\text { ZiiP "Mango" } \\
\text { Pacha Mama Salts }\end{array}$ & 5.0 & 3.5 & 1.8 & 3.7 & 0.00 & $\mathbf{U}$ & N/A & JUUL "Mango" \\
\hline $\begin{array}{l}\text { "Fuji" } \\
\text { Salt Bae } 50 \text { "Blue }\end{array}$ & 4.2 & 4.2 & 2.2 & $\mathbf{U}$ & 0.08 & 0.09 & $0.08 \pm 0$ & PG+GL+Nicotine ${ }^{\ddagger}$ \\
\hline Raspberry & & & & & & & & Salt Bae ${ }^{50}$ "Blue \\
\hline $\begin{array}{l}\text { Lemonade" } \\
\text { JUUL "Virginia }\end{array}$ & 4.2 & 4.1 & 2.1 & $\mathbf{U}$ & 0.01 & 0.01 & $0.01 \pm 0.01$ & Raspberry Lemonade" \\
\hline Tobacco" & 3.0 & 3.0 & 1.6 & 1.0 & 0.14 & 0.14 & $0.14 \pm 0$ & JUUL "Mango" \\
\hline $\begin{array}{l}\text { JUUL "Mint" } \\
\text { Pacha Mama Salts }\end{array}$ & 3.0 & 3.0 & 1.6 & 1.1 & 0.11 & 0.10 & $0.11 \pm 0.01$ & JUUL "Mango" \\
\hline $\begin{array}{l}\text { "Fuji" } \\
\text { Salt Bae } 50 \text { "Blue } \\
\text { Raspberry }\end{array}$ & 2.1 & 2.0 & 1.0 & U & 0.08 & 0.08 & $0.08 \pm 0$ & $\begin{array}{l}\text { PG+GL+Nicotine }{ }^{\ddagger} \\
\text { Salt Bae }{ }^{50} \text { "Blue } \\
\text { Raspberry Lemonade" }\end{array}$ \\
\hline $\begin{array}{l}\text { Lemonade" } \\
\text { Fuzion Vapor }\end{array}$ & 2.1 & 2.1 & 1.1 & $\mathbf{U}$ & 0.02 & 0.01 & $0.02 \pm 0.01$ & \\
\hline $\begin{array}{l}\text { "Ectoplasm" } \\
\text { Fuzion Vapor } \\
\text { "Roundhouse with }\end{array}$ & 2.0 & 1.0 & 0.5 & $\mathbf{U}$ & $\mathbf{U}$ & 0.98 & N/A & PG+GL+Nicotine f $^{\ddagger}$ \\
\hline $\begin{array}{l}\text { Cream" } \\
\text { Fuzion Vapor }\end{array}$ & 2.0 & 2.8 & 1.6 & U & 0.68 & 0.71 & $0.70 \pm 0.01$ & PG+GL+Nicotine ${ }^{\ddagger}$ \\
\hline $\begin{array}{l}\text { "Unicorn Blood" } \\
\text { Fuzion Vapor }\end{array}$ & 2.0 & 0.9 & 0.5 & $\mathbf{U}$ & 0.82 & 0.86 & $0.84 \pm 0.02$ & PG+GL+Nicotine F $^{\ddagger}$ \\
\hline "Ectoplasm" & 0.5 & 0.5 & 0.3 & U & 0.95 & 0.97 & $0.96 \pm 0.01$ & PG+GL+Nicotine ${ }^{\ddagger}$ \\
\hline
\end{tabular}




\begin{tabular}{|c|c|c|c|c|c|c|c|c|}
\hline \multirow[b]{2}{*}{ Brand "Flavor" } & \multicolumn{3}{|c|}{ Nicotine Content } & \multirow[b]{2}{*}{$\begin{array}{c}\text { Molar Ratio } \\
\text { Acid/Nicotine }\end{array}$} & \multirow[b]{2}{*}{$\begin{array}{c}\alpha_{\mathrm{fb}} \text { using } \\
\mathrm{H}_{\mathrm{a}}\end{array}$} & \multirow[b]{2}{*}{$\begin{array}{c}\alpha_{\mathrm{fb}} \text { using } \\
\mathrm{H}_{\mathrm{b}}\end{array}$} & \multirow[b]{2}{*}{ Average $\alpha_{\mathrm{fb}}$} & \multirow[b]{2}{*}{$\begin{array}{l}\text { E-liquid used to prepare } \\
\text { proton-shift references } \\
\text { for calculating } \alpha_{\mathrm{fb}} \underline{\S}\end{array}$} \\
\hline & $\begin{array}{l}\text { Listed } \\
\text { (wt\%) }\end{array}$ & $\begin{array}{l}\text { Actual } \\
\text { (wt\%) }\end{array}$ & $\begin{array}{l}\text { Actual } \\
\text { (mol\%) }\end{array}$ & & & & & \\
\hline \multicolumn{9}{|l|}{$\begin{array}{l}\text { Fuzion Vapor } \\
\text { "Unicorn Blood }\end{array}$} \\
\hline $\begin{array}{l}\text { Prime" } \\
\text { Fuzion Vapor }\end{array}$ & 0.5 & 0.4 & 0.2 & U & 0.50 & 0.55 & $0.53 \pm 0.02$ & PG+GL+Nicotine ${ }^{\ddagger}$ \\
\hline $\begin{array}{l}\text { "Unicorn Blood" } \\
\text { Fuzion Vapor } \\
\text { "Roundhouse with }\end{array}$ & 0.5 & 0.4 & 0.2 & $\mathbf{U}$ & 0.42 & 0.44 & $0.43 \pm 0.01$ & PG+GL+Nicotine $e^{\ddagger}$ \\
\hline Cream" & 0.5 & 0.5 & 0.3 & U & 0.07 & 0.09 & $0.08 \pm 0.01$ & $P G+G L+$ Nicotine $e^{\ddagger}$ \\
\hline
\end{tabular}

* = Samples contained levulinic acid rather than benzoic acid (all other acids).

คे $\mathrm{U}=$ Undetected because the resonance(s) required for this calculation were not present, not resolved, or were too broad for analysis.

$\mathrm{N} / \mathrm{A}=$ Not applicable because only one $\alpha_{\mathrm{fb}}$ value was calculated.

$\S=$ Acid and base references refer to the monoprotonated and free-base nicotine forms, respectively.

$\$=$ Lab-prepared propylene glycol $(\mathrm{PG})+$ glycerol $(\mathrm{GL})+59 \mathrm{mg} / \mathrm{mL}$ nicotine mixture 


\section{Conclusions}

This dissertation includes four manuscripts ${ }^{1,2,3,4}$ covering topics relevant to electronic cigarette use and public health. These topics include the boiling points of propylene glycol (PG) and glycerol (GL) mixtures (with and without water and nicotine, respectively), effect of sucralose on e-liquid degradation during vaping, exploration of the protonation state of nicotine in e-liquids (free-base fraction, $\alpha_{\mathrm{fb}}$ ), and acid/nicotine ratios in e-liquids coupled with the concentration of nicotine in commercial e-liquids vs. the listed nicotine content. The goal of determining the boiling points of simple e-liquids was to find the necessary temperature range for the vaporization of e-liquids; application of excess energy (in the form of a higher temperature) increases the likelihood that degradation occurs during vaping. The analysis of the effect of sucralose on degradation trends produced by vaping $\mathrm{PG}+\mathrm{GL}$ mixtures aimed to isolate the effect of a single variable, sucralose, on degradation in the hopes of identifying/removing variables that can contribute to increased harm potential. The exploration of nicotine in e-liquids $\left(\alpha_{\mathrm{fb}}\right.$, acid identification, acid/nicotine ratio, and nicotine concentration) was done with the aim of achieving a greater understanding of the nicotine chemistry within e-liquids. As an important component of many e-liquids, most of these variables (i.e. $\alpha_{\mathrm{fb}}$, added acid, and acid/nicotine ratio) are not widely advertised, with the exceptions of the nicotine content and the advertisement of some e-liquids as "nicotine salts". The ratio of acid/nicotine in e-liquids and $\alpha_{\mathrm{fb}}$ are generally not advertised, yet can greatly impact the consumer experience in terms of potential harshness, as well as the ability to detect higher levels of nicotine (if there is a lack of harshness). Since nicotine is the main addictive component 
in e-liquids, it is important to understand how nicotine's chemistry can be manipulated in order to modify the vaping experience and possibly addiction potential. Herein, the broader findings and implications of these manuscripts are explored.

6.1. Boiling Points of the Propylene Glycol + Glycerol System at 1 Atmosphere Pressure: $188.6-292^{\circ} \mathrm{C}$ Without and With Added Water or Nicotine ${ }^{1}$

To begin the exploration of e-cigarette chemistry, the boiling points of PG+GL mixtures were explored and the Gibbs-Konovalov theorem ${ }^{5,6}$ was used to generate equations to predict boiling point trends for PG+GL mixtures based on the composition (by mole, volume, or weight $\%$ ). Traditionally, the Antoine equation ${ }^{7}$ can be applied to mixtures to determine an approximate boiling point, but the boiling points for PG and GL differed by so much (over $100^{\circ} \mathrm{C}$ ) that the ranges for their Antoine parameters do not completely cover the range of temperatures needed (the parameterizations for PG were the limiting variable here, which only went up to $188.3^{\circ} \mathrm{C}$, while pure GL boils at 292 $\left.{ }^{\circ} \mathrm{C}\right)$. The effect of adding $2.5 \mathrm{~mol} \%$ water, $5 \mathrm{~mol} \%$ water, and $3 \mathrm{~mol} \%$ nicotine on PG+GL mixture boiling points was also determined. Added water reduced the boiling points of all mixtures tested, and the $5 \mathrm{~mol} \%$ water condition decreased the boiling points more than the $2.5 \mathrm{~mol} \%$ water condition. Added nicotine, which boils around $247^{\circ} \mathrm{C}$, was associated with a lowering of boiling points for mixtures containing > $75 \mathrm{~mol} \%$ GL.

This Gibbs-Konovalov parametrization of PG+GL mixture boiling points is useful for consumers and researchers who can now estimate the boiling point of any mixture to find a suitable vaporization temperature. The e-liquid must be heated to its boiling point (or higher) in order to vaporize/vape the e-liquid. Significantly heating an e-liquid above its boiling point can result in excess energy being applied to the system and, as a result, 166 
the components can degrade during vaping. Degradation is an undesirable phenomenon during vaping as the compounds that tend to form (mainly aldehydes) can cause respiratory irritation, inflammation, or worse, as smaller amounts of carcinogenic compounds such as benzene and formaldehyde can be formed as well.

In the future, it would be useful to further explore the interaction between e-liquid boiling points, vaping temperatures in e-cigarettes, and degradation production/concentrations. More methods should be developed that allow coil temperatures during vaping to be evaluated for a range of devices. The temperature achieved by an e-liquid during vaping, whether while being vaporized, or while wicking into the coil wicking material, should be emphasized when studying e-liquid degradation. Furthermore, new coil designs should be explored to evaluate possible attempts to minimize the temperatures reached by e-liquid not actively being vaporized, including more efficient wicking.

6.2. Sucralose-Enhanced Degradation of Electronic Cigarette Liquids during Vaping ${ }^{2}$ Many variables can influence degradation produced during vaping. As discussed above, the boiling points of e-liquids can theoretically influence degradation if excessive heating occurs (even if just localized). Following the exploration of the boiling points of e-liquids with different compositions, the effect of an individual variable, sucralose, on degradation was determined during vaping. This research was performed with the goal of elucidating the impact of sucralose on e-liquid degradation during vaping. 
The concentration of sucralose was calculated for a number of commercially available e-liquids using ${ }^{1} \mathrm{H}$ NMR data. These values were used to establish similar concentrations used for lab-prepared e-liquids that were then vaped; lab-prepared eliquids contained: PG, GL, and sometimes nicotine and sucralose. NMR samples, discussed below, were vaped at 20 watts (within the limits recommended by the manufacturer) using the CORESTA puff method $^{8}$ and a $1.2 \Omega$ organic cotton coil (OCC). Vaped samples were first tested for degradation level consistency within a condition by ${ }^{1} \mathrm{H}$ NMR. Degradation was consistent within a condition; this established that the degradation effects observed were not simply due to "coil aging". Consistency in degradation levels, relative to PG, was found for both no sucralose samples and samples generated using e-liquid containing a "higher" sucralose concentration $(0.10 \mathrm{~mol} \%$ sucralose). Differences in the concentration of degradation produced from vaping were found for 3 coils (same manufacturer, resistance, and model) using ${ }^{1} \mathrm{H}$ NMR. This indicated that it can be difficult to generalize degradation production because different coils can produce different ranges of degradation during vaping. Regardless of the initial degradation produced by a coil, all coils showed the same general trend by ${ }^{1} \mathrm{H}$ NMR: an increased sucralose concentration resulted in increased degradation production, as indicated by measurement of aldehydes (propanal, acetaldehyde, glycolaldehyde, acrolein, and PG/GL + formaldehyde hemiacetals) resulting from the aerosolization process.

Previous research indicated sucralose is thermally unstable and that the breakdown of sucralose can generate hydrochloric acid. ${ }^{9,10}$ Therefore, we hypothesized 
that sucralose could be promoting degradation production by acid generation and potentially via acid catalysis. To test this, nicotine was added to sucralose mixtures to evaluate the free-base fraction relative to monoprotonated nicotine $\left(\alpha_{\mathrm{fb}}\right)$ before and after vaping. Nicotine added to the e-liquids $(24 \mathrm{mg} / \mathrm{mL} \approx 1 \mathrm{~mol} \%)$ was in the free-base state before vaping $\left(\alpha_{\mathrm{fb}} \approx 1\right)$ for mixtures without and with sucralose $(0.12 \mathrm{~mol} \%)$ before vaping. After vaping these e-liquids, $\alpha_{\mathrm{fb}}=0.96$ for the sucralose-free sample $\left(\alpha_{\mathrm{fb}} \approx 1\right)$, and $\alpha_{\mathrm{fb}}=0.75$ for the vaped sucralose-containing e-liquid $(0.12 \mathrm{~mol} \%$ sucralose before vaping). This indicated that more acid was produced during vaping using the sucralosecontaining e-liquid because $\alpha_{\mathrm{fb}}$ decreased more after vaping compared to the unvaped eliquid. It was also determined that $\sim 2.2$ protons were absorbed by nicotine based on the change in $\alpha_{\mathrm{fb}}$ after vaping; this indicates the total amount of acid absorbed by nicotine, not just the acid produced due to sucralose breakdown. PG and GL can also be sources of small amounts of acid during vaping. This value is consistent with proposed mechanisms of sucralose pyrolysis, which indicated that each sucralose could produce two equivalents of hydrochloric acid. ${ }^{10} \mathrm{PG} / \mathrm{GL}$ degradation during vaping can produce acids, including acetic acid, which could contribute to the slightly larger value of 2.2 equivalents of acid relative to each equivalent of sucralose obtained herein.

To confirm that sucralose was degrading during vaping, and not just catalyzing degradation while intact, vaped e-liquid samples were also tested using ion chromatography (IC) to determine if free chloride was generated during vaping (sucralose was the only chloride-containing compound in the e-liquid). IC results did indicate that free chloride was present in the vaped sample, and accounted for $\sim 30 \%$ of 
the total chlorine in the original sample. By IC, it was estimated that $\sim 0.9$ free chlorides were released per sucralose during vaping. Sucralose was not stable in this vaping environment and so decomposed. Other degradation products that originated from sucralose (as they are chlorine-containing) included: 3-chloropropan-1,2-diol (determined by gas chromatography-flame ionization detector, GC/FID) and 1,6-dichloro-1,6dideoxyfructose (determined by gas chromatography-mass spectrometry, GC/FID); when combined, these products accounted for $\sim 1 \%$ of the total chlorine expected in the sample. Sucralose was not detected in the vaped samples, supporting its lack of volatility.

Other degradation products detected by GC/FID, GC/MS, or IC included: isopropyl alcohol + formaldehyde hemiacetal, 4-methyl-1,3-dioxolane, 4-hydroxymethyl1,3-dioxolane, 5-hydroxy-1,3-dioxane, 4-hydroxymethyl-2-methyl-1,3-dioxolane, acetaldehyde diisopropyl acetal, $\beta$-levoglucosan, and an unidentified chlorinated compound.

To our knowledge, this is the first study to report that sucralose can enhance degradation production, in particular aldehydes, during e-cigarette vaping. We report that sucralose was unstable during vaping in the e-cigarettes we tested. As such, sucralose should be disclosed as an e-liquid ingredient so that consumers can make an informed choice. Due to the non-volatile nature of sucralose and its ability to enhance degradation production (which is undesirable for health reasons), it is recommended that consumers avoid vaping sucralose-containing e-liquid.

In the future, it would be beneficial to test if the same level of increased degradation due to sucralose is observed for other e-cigarettes and determine sucralose 
prevalence in commercial e-liquids. The effects of individual e-liquid components on degradation production during vaping should also be determined in the future. Flavorants in general are known to enhance the production of aldehydes during vaping, ${ }^{11}$ but the effects of many individual flavorants (present in e-liquids) on degradation during ecigarette vaping remains unknown.

\subsection{Free-Base Nicotine Determination in Electronic Cigarette Liquids by ${ }^{1} \mathrm{H}$ NMR Spectroscopy ${ }^{3}$}

As discussed in the last section, individual components of e-liquids (e.g. sucralose) can drastically alter the vaping experience and potentially level of harm (e.g. by increasing degradation production in the form of aldehydes). Another component commonly included in e-liquids that can affect the vaping experience and possibly the harm of vaping is nicotine. Nicotine is included in a variety of e-liquids in different concentrations, typically not exceeding $\sim 60 \mathrm{mg} / \mathrm{mL}$. As discussed previously, depending on the $\mathrm{pH}$ of the medium, nicotine can exist in three protonation states, only two of which are typically relevant for tobacco products: free-base (unprotonated) and monoprotonated. Free-base nicotine exists in both the gas phase and the particulate phase, while monoprotonated nicotine can only exist in the particulate phase. This equilibrium has been suggested to have implications for impact/harshness and addiction potential. ${ }^{12}$ This ratio of free-base nicotine relative to monoprotonated nicotine is represented by $\alpha_{\mathrm{fb}}$. Since the free-base form is mainly responsible for the impact/harshness, as $\alpha_{\mathrm{fb}}$ decreases, the concentration of nicotine that can be tolerated by the consumer (based on harshness) increases. 
For this set of experiments, ${ }^{1} \mathrm{H}$ NMR was applied for the first time (to the best of our knowledge) to e-liquids to determine $\alpha_{\mathrm{fb}}$. Others have attempted to determine $\alpha_{\mathrm{fb}}$ of eliquids using other methods, typically using the addition of solvents (e.g. water), which can alter the chemistry/equilibrium. The NMR method described herein allows the determination of $\alpha_{\mathrm{fb}}$ without the addition of solvent to the sample. The NMR lock solvent is placed in an outer NMR tube, and the unmodified (neat) e-liquid sample is placed in an inner concentric tube. This allows $\alpha_{\mathrm{fb}}$ to be determined for an unperturbed system, resulting in more accurate $\alpha_{\mathrm{fb}}$ values. In addition to accuracy benefits, this method is also fast ( 2 minutes of NMR acquisition time) once reference standards have been established. The method described herein is based on ${ }^{1} \mathrm{H}$ NMR resonance peak averaging, where the chemical shifts for the two protonation states of nicotine in the mixture average together when the proton exchange rate between the two forms is greater than the chemical shift difference in $\mathrm{Hz}$. This allows a single set of nicotine resonances for a sample to be compared with free-base and monoprotonated nicotine standards to calculate $\alpha_{\mathrm{fb}}$ from the chemical shifts.

This NMR method was applied to 11 commercially available e-liquids to determine $\alpha_{\mathrm{fb}}$. Two of these e-liquids were "salt nicotine" pods produced by currently very popular ${ }^{13}$ JUUL to be used with a JUUL device and 9 were "non-salt" nicotine eliquids for refillable e-cigarette systems. $\alpha_{\mathrm{fb}}$ ranged from 0.03 to 0.84 , and ${ }^{1} \mathrm{H}$ NMR determined nicotine concentrations ranged from 3 to $58 \mathrm{mg} / \mathrm{mL}$. Most of the e-liquids (i.e., 6) had $\alpha_{\mathrm{fb}} \geq 0.68$, indicating nicotine was primarily in the harsher free-base form for the non-salt e-liquids; the nicotine concentration for these e-liquids was $\leq 27 \mathrm{mg} / \mathrm{mL}$. The 
two JUUL salt nicotine products tested had $\alpha_{\mathrm{fb}} \approx 0.1$, with nicotine concentrations of 56 and $58 \mathrm{mg} / \mathrm{mL}$, as determined by ${ }^{1} \mathrm{H}$ NMR. The only non-salt nicotine e-liquid with $\alpha_{\mathrm{fb}}$ in the range of JUUL products contained $3 \mathrm{mg} / \mathrm{mL}$ nicotine; this is important because very little acid is needed to alter $\alpha_{\mathrm{fb}}$ for such a small nicotine concentration compared to the high nicotine content in JUUL products. It is possible that acidic flavorants were added to this e-liquid, and happened to alter $\alpha_{\mathrm{fb}}$.

As was mentioned above, solvent dilution (i.e., dilution of the e-liquid into water prior to $\mathrm{pH}$ measurement) is not an accurate way to determine $\alpha_{\mathrm{fb}}$ for nicotine-containing products. To demonstrate that solvent dilution is a less valid method than this direct "neat" NMR method, $\mathrm{D}_{2} \mathrm{O}$ (deuterated water) was also added to a JUUL e-liquid and tested by ${ }^{1} \mathrm{H}$ NMR to determine the extent to which the nicotine protonation state $\left(\alpha_{\mathrm{fb}}\right)$ was altered. The JUUL e-liquid in its neat from had $\alpha_{\mathrm{fb}} \approx 0.1$; when diluted with 5 parts water relative to 1 part e-liquid (by volume), $\alpha_{\mathrm{fb}}=0$ (entirely monoprotonated). This illustrated that when determining the protonation state of an e-liquid, dilution in water (simulated using $\mathrm{D}_{2} \mathrm{O}$ in this instance) would lead to $\alpha_{\mathrm{fb}}=0$, rather than 0.1 , which can have different implications for consumer perception and potentially addiction. In order to obtain the most accurate $\alpha_{\mathrm{fb}}$ values, methods that involve solvent dilution (e.g. pH determinations) should be avoided. The simple and fast ${ }^{1} \mathrm{H}$ NMR method described herein is an efficient way of determining $\alpha_{\mathrm{fb}}$ for e-liquids.

In the future, it is recommended that $\alpha_{\mathrm{fb}}$ for e-liquids be considered as well when reporting nicotine content due to its influence on the consumer experience and on product impact/harshness. Consumers should be informed of the $\alpha_{\mathrm{fb}}$ of their products on e-liquid 
labels. Also, when considering tobacco product regulation, $\alpha_{\mathrm{fb}}$ should be examined in addition to the nicotine concentration as the two can control consumer perception, in particular when it comes to harshness. As mentioned previously, an e-liquid with low $\alpha_{\mathrm{fb}}$ can contain a greater concentration of nicotine and still be tolerable for inhalation (i.e. not too harsh) compared to an e-liquid with a greater $\alpha_{\mathrm{fb}}$, which may be harsher with lower nicotine concentration. When a greater concentration of nicotine is easier to inhale, the eliquid potentially becomes more addictive than an e-liquid containing a lower concentration of nicotine, even if the two products have similar levels of harshness.

\subsection{Nicotine in Tobacco Product Aerosols: "It's Déjà Vu All Over Again"4}

In the last section, a direct, non-destructive, and fast NMR method had been developed for determining $\alpha_{\mathrm{fb}}$ in e-liquids (Section 6.3, above). This method can now be applied to analyze a greater variety of e-liquids, in particular, those of different types (pods vs bottles, "salt nicotine" vs non-salt nicotine). In this manuscript, $\alpha_{\mathrm{fb}}$, the nicotine concentration $(\mathrm{mg} / \mathrm{mL})$, added acid, and molar acid/nicotine ratio were determined for 29 e-liquids including: a full selection of JUUL pods available at the time of purchase ("salt nicotine" e-liquids), other JUUL compatible "salt nicotine" pods (ZiiP and Eon Smoke), other "salt nicotine" e-liquid pods, "nicotine salt" bottled e-liquids, and non-salt e-liquid bottles. The trajectory of e-liquid production over the years, in terms of $\alpha_{\mathrm{fb}}$ and nicotine concentration, were then compared to the trajectory of traditional cigarettes.

The initial e-liquids that were released for e-cigarettes were not advertised as nicotine "salts" and thus had higher $\alpha_{\mathrm{fb}}$ levels. When nicotine is commercially purchased, it comes in the free-base form. Acid must then be added to the nicotine to convert it into 
the "salt" form, as desired. Due to the higher $\alpha_{\mathrm{fb}}$ levels of older generation e-liquids, the associated nicotine concentrations were relatively low, due to the harshness of free-base nicotine compared to monoprotonated nicotine. Higher nicotine levels could not be tolerated by the consumer with higher $\alpha_{\mathrm{fb}}$.

Acids were not identified in the non-salt e-liquids (x7). Nicotine ranged from 0.4 to 2.8 weight $\%$ nicotine (or 5 to $33 \mathrm{mg} / \mathrm{mL}$ nicotine). $\alpha_{\mathrm{fb}}$ ranged from 0.08 to 0.98 . The same flavors of e-liquids were purchased in different nicotine concentrations and $\alpha_{\mathrm{fb}}$ was found to vary linearly with nicotine concentration. In general, it seemed that greater nicotine concentrations were associated with higher $\alpha_{\mathrm{fb}}$, and lower nicotine concentrations were associated with lower $\alpha_{\mathrm{fb}}$. We theorized that this could inadvertently be due to the presence of acidic flavorants. Unsurprisingly, lower nicotine concentrations are more easily influenced by the acidity/basicity of flavorants than greater nicotine concentrations.

Nicotine "salt" bottled e-liquids $(\mathrm{x} 4)$ were found to have nicotine concentrations ranging from 2.0 to 4.2 weight $\%$ ( 24 to $50 \mathrm{mg} / \mathrm{mL}$ nicotine). $\alpha_{\mathrm{fb}}$ ranged from 0.01 to 0.08 for the "salt-nicotine" bottled e-liquids. This lower $\alpha_{\mathrm{fb}}$ allows the greater nicotine concentrations to be tolerated and not be excessively harsh when the aerosols are inhaled. The acids used to alter $\alpha_{\mathrm{fb}}$ were not identified in these "salt-nicotine" bottled e-liquids.

JUUL pod e-liquids (x10) were ordered in all flavors and nicotine concentrations available at the time of purchase. Two e-liquids came in 3 weight $\%$ nicotine and all others were 5 weight $\%$ nicotine. $\alpha_{\mathrm{fb}}$ for all JUUL e-liquids ranged from 0.09 to 0.14 . The average $\alpha_{\mathrm{fb}}( \pm \mathrm{SD})$ for all JUUL e-liquids was $0.12 \pm 0.01$. All JUUL e-liquids were found 
to contain 1:1 benzoic acid:nicotine by mol. For the e-liquids advertised as 3 weight $\%$ nicotine, this was confirmed by NMR, finding 3.0 weight $\%$ nicotine (or $35 \mathrm{mg} / \mathrm{mL}$ nicotine) relative to PG and GL. For the e-liquids advertised as 5 weight $\%$, the nicotine content determined by NMR ranged from 4.9 to 5.2 weight\% (or 58 to $62 \mathrm{mg} / \mathrm{mL}$ nicotine), relative to PG and GL.

For all other "salt nicotine" pod e-liquids, $\alpha_{\mathrm{fb}}$ ranged from 0.00 to 0.19 , and the nicotine concentrations ranged from 2.3 to 4.8 weight\% (or 27 to $57 \mathrm{mg} / \mathrm{mL}$ nicotine) as determined by NMR. All of the "salt nicotine" pod e-liquids contained either benzoic acid or levulinic acid, as determined by NMR. The acid/nicotine molar ratio ranged from 0.22 to 4.03 for these "salt nicotine" pod e-liquids. The nicotine concentration for these eliquids often differed from the reported nicotine concentration, all of which contained less than advertised, and one even contained less than half of the advertised nicotine.

Overall, it appears that nicotine-containing e-liquids have, over time, shifted from higher $\alpha_{\mathrm{fb}}$ and lower nicotine content, to lower $\alpha_{\mathrm{fb}}$ and higher nicotine content. Bottled "non-salt" nicotine e-liquids were available first, followed by JUUL, other salt-nicotine pods, and then nicotine-salt bottled e-liquids. The $\alpha_{\mathrm{fb}}$ trajectory that occurred for ecigarettes mirrors the historical cigarette $\alpha_{\mathrm{fb}}$ changes that occurred during the 1900s, which was responsible for the addictiveness and deadliness of the modern cigarette.

Cigarettes were originally harsher and had higher $\alpha_{\mathrm{fb}}$. However, due to the development of different tobacco drying methods that were applied, the $\alpha_{\mathrm{fb}}$ content in cigarettes decreased over time, approaching $\alpha_{\mathrm{fb}} \approx 0$. This progression produced a much milder tobacco smoke that was less harsh and thus easier to inhale deeply into the lungs. 
While easier to inhale, these milder cigarettes had less of an "impact" on the user than cigarettes with slightly higher $\alpha_{\mathrm{fb}}$. The tobacco industry then partially "re-freebased" the nicotine in cigarettes to regain some of the impact originally imparted to cigarettes with some free-base nicotine and settled on $\alpha_{\mathrm{fb}} \approx 0.1$. The inhalability of these cigarettes and the "hit" generated from $\alpha_{\mathrm{fb}} \approx 0.1$ resulted in both the addictiveness and the harm that can be generated from their use.

As discussed, e-liquids followed a similar path, where some of the first e-liquids commercially available, typically sold in bottles, had higher $\alpha_{\mathrm{fb}}$, and over time, $\alpha_{\mathrm{fb}}$ was reduced to $\sim 0.1$ (with the introduction of JUUL pods/products, which presently dominate the e-cigarette market based on market share). While this trajectory likely has made modern e-liquids such as those sold by JUUL with $\alpha_{\mathrm{fb}} \approx 0.1$ and high nicotine content ( $\sim 60 \mathrm{mg} / \mathrm{mL}$ nicotine) more satisfying for former/current cigarette smokers, this has also resulted in a potentially much more addictive product for never-smokers.

Based on this research, it is recommended that $\alpha_{\mathrm{fb}}$ of e-liquids be monitored in addition to the nicotine concentration. These two variables combine to determine the total harshness of a product when vaped. Since harshness can possibly indicate to the consumer that they are vaping a certain amount of nicotine and regulate their vaping behavior, changes to $\alpha_{\mathrm{fb}}$ can interfere with a consumer's ability to adjust intake based on desired nicotine. $\alpha_{\mathrm{fb}}$ and nicotine concentration should be analyzed for all e-liquid products and should be considered for e-cigarette regulation policies. 


\subsection{Overall Conclusions}

This document has detailed some of the variables that contribute to the understanding of e-cigarette chemistry. First, the boiling points of simple PG/GL eliquids were explored to determine the temperature ranges required for e-liquid vaporization. Devices that vastly exceed these expected temperature ranges are likely to result in excessive heating (even if just localized) and thus greater concentrations of degradation. To explore how degradation during vaping can be impacted by a single variable, experiments using varying concentrations of sucralose were conducted. Sucralose was found to be unstable in the e-cigarettes tested and that greater sucralose concentrations increased the amount of degradation produced (primarily aldehydes) during vaping. These findings indicated that sucralose should not be vaped in e-cigarettes and indicated that there is a possibility that other components used in e-liquids could be enhancing the amount of degradation produced by an e-cigarette. Degradation is undesirable for health reasons and should be minimized when possible. The role of other e-liquid additives/flavorants in controlling degradation production should be explored to determine chemicals that are unsuitable for vaping. Another e-liquid variable that needs to be analyzed for commercially available e-liquids is nicotine. The nicotine concentration, $\alpha_{\mathrm{fb}}$, the presence of acids, and the acid/nicotine ratio for products should be included in e-liquid analyses. As such, an NMR method was developed to calculate $\alpha_{\mathrm{fb}}$ for neat e-liquids. This method was then applied to analyze $\alpha_{\mathrm{fb}}$ of e-liquids over time as products have shifted from tanks (filled with bottled e-liquids) to pod-based systems, such as JUUL, which take pods. This analysis revealed that e-liquids have followed a 
similar $\alpha_{\mathrm{fb}}$ trajectory as was seen for traditional cigarettes in the United States in the 1900s, where products initially have high $\alpha_{\mathrm{fb}}$, and shift to $\alpha_{\mathrm{fb}} \approx 0.1$, which allows the resulting aerosol to be less harsh than a higher $\alpha_{\mathrm{fb}}$. These findings have implications for the addiction potential of pod-based e-cigarettes, particularly when low $\alpha_{\mathrm{fb}}$ is combined with a high nicotine concentration. 


\subsection{References}

${ }^{1}$ Duell, Anna K., James F. Pankow, Samantha M. Gillette, and David H. Peyton. "Boiling points of the propylene glycol+ glycerol system at 1 atmosphere pressure: 188.6-292 ${ }^{\circ} \mathrm{C}$ without and with added water or nicotine." Chemical Engineering Communications 205, no. 12 (2018): 1691-1700.

2 Duell, Anna K., Kevin J. McWhirter, Tetiana Korzun, Robert M. Strongin, and David H. Peyton. "Sucralose-enhanced degradation of electronic cigarette liquids during vaping." Chemical Research in Toxicology 32, no. 6 (2019): 1241-1249.

${ }^{3}$ Duell, Anna K., James F. Pankow, and David H. Peyton. "Free-base nicotine determination in electronic cigarette liquids by ${ }^{1} \mathrm{H}$ NMR spectroscopy." Chemical Research in Toxicology 31, no. 6 (2018): 431-434.

${ }^{4}$ Duell, Anna K., James F. Pankow, and David H. Peyton. "Nicotine in Tobacco Product Aerosols: "It's Déjà Vu All Over Again"§" Tobacco Control. Accepted manuscript.

${ }^{5}$ Al-Jiboury, K. F. C. (2007). Correlation for fitting multicomponent vapor-liquid equilibria data and prediction of azeotropic behavior, Al-khwarizmi Eng. J., 3, $67-86$.

${ }^{6}$ Malesinski, W. (1965). Azeotropy and Other Theoretical Problems of Vapour-liquid Equilibrium, Interscience Publishers.

7 Thomson, George Wm. "The Antoine equation for vapor-pressure data." Chemical Reviews 38, no. 1 (1946): 1-39.

${ }^{8}$ CORESTA; CORESTA Recommended Method Number 81: Routine Analytical Machine for E-Cigarette Aerosol Generation and Collection -Definitions and Standard Conditions. (2015). [Internet]. [accessed 2019 October 14]. Available from: https://www.coresta.org/sites/default/files/technical_documents/main/CRM_81.pdf

${ }^{9}$ De Oliveira, Diogo N., Maico De Menezes, and Rodrigo R. Catharino. "Thermal degradation of sucralose: a combination of analytical methods to determine stability and chlorinated byproducts." Scientific Reports 5 (2015): 9598.

${ }^{10}$ Rahn, Anja, and Varoujan A. Yaylayan. "Thermal degradation of sucralose and its potential in generating chloropropanols in the presence of glycerol." Food Chemistry 118, no. 1 (2010): 56-61.

${ }^{11}$ Khlystov, Andrey, and Vera Samburova. "Flavoring compounds dominate toxic aldehyde production during e-cigarette vaping." Environmental Science \& Technology 50, no. 23 (2016): 13080-13085. 
${ }^{12}$ Pankow, James F. "A consideration of the role of gas/particle partitioning in the deposition of nicotine and other tobacco smoke compounds in the respiratory tract." Chemical Research in Toxicology 14, no. 11 (2001): 1465-1481.

${ }^{13}$ King, Brian A., Doris G. Gammon, Kristy L. Marynak, and Todd Rogers. "Electronic cigarette sales in the United States, 2013-2017." JAMA 320, no. 13 (2018): 1379-1380. 